\title{
THE FERMILAB ANTIPROTON SOURCE DESIGN REPORT
}

\section{FEBRUARY 1982}

Fermi National Accelerator Laboratory Batavia Illinois

- Operated by Universities Research Association Inc., under contract with the U.S. Department of Energy

Revised Feb. 26,1982 
1. Introduction and Overview

2. Proton Acceleration

3. Targeting and Transport

3.1 Main Ring Extraction

3.2 Proton Transport

3.3 Targeting and Antiproton Production

3.3.1 Production Cross Sections

3.3.2 Proton Energy

3.3.3 Antiproton Momentum

3.3.4 Antiproton Longitudinal Acceptance

3.3.5 Antiproton Transverse Acceptance

3.3.6 Targeting Limitations

3.3.7 The Lithium Lens Collector

3.3.8 Alternative Collectors

3.4 Antiproton Target

3.4.1 Material Choice

3.4.2 Energy Deposition

3.4.3 Target Design

3.5 Target Hall

3.6 Antiproton Transport to the Debuncher

4. Debuncher Ring

4.1 Purpose of the Debuncher

4.2 Rotation and Debunching

4.3 The Debuncher Ring Lattice

4. 4 Beam Transfer

4.4.1 Injection

4.4.2 Extraction

4.5 Radio Frequency Systems

4.5.1 Antiproton Debuncher RF

4.5.2 Gap-Preserving RF

4.6 Betatron Cooling

4.7.1 Cooling System Parameters

4.7.2 Pickup and Kicker Design

4.7.3 Effect of the Gap on Betatron Cooling

4.8 Equipment Arrangement

5. Accumulator Ring

5.1 Stochastic Cooling in the Accumulator

5.1.1 Introduction and Summary of the Stochastic Stacking Process

5.1 .2 Summary of Design Considerations

5.1.3 Building the Exponential Gain Profile

5.1 .4 Signal Suppression and Stability

5.1 .5 Core Cooling

5.1.6 Numerical Calculations of Momentum Cooling

5.1.7 Betatron Cooling

5.1.8 Stochastic-Cooling Hardware

5.2 RF Stacking System 
5.3 Accumulator Lattice

5.4 Accumulator Vacuum System

5.4.1 Vacuum Requirements

5.4.2 Vacuum System Layout and Characteristics

5.5 Accumulator Magnets

6. Extraction of Antiprotons from the Accumulator Core

and Acceleration to $1 \mathrm{TeV}$

6.1 Extraction

6.1 .1 Introduction

6.1 .2 Accumulator Beam Manipulation

6.2 Acceleration

6.2.1 Introduction

6.2.2 Injection and Rebunching

6.2.3 Bunch Recombination at $150 \mathrm{GeV}$

6.2.4 Transfer of Beam from Main Ring to Tevatron

6.2.5 Completion of Filling

6.2.6 Tevatron RF Considerations

6.3 Tolerances and Beam Lifetime

6.4 Beam Abort

7. Interaction Region

7.1 Quadrupoles

7.2 Power Supply and Bus

7.3 Refrigeration

7.4 Vacuum

8. Performance and Luminosity

8.1 Beam Geometry

8.2 Beam Cross Section at the Collision Point

8.3 Luminosity

8.4 Beam-Beam Tune Shift

8.5 Single-Beam and Luminosity Lifetime

8.5.1 Effects of Residual Gas

8.5.2 Intrabeam Scattering

8.5.3 Beam-Beam Effects

8.6 Beam-Stability Considerations in the Tevatron

9. Commissioning and Operation; Diagnostics and Controls

9.1 Commissioning

9.2 Diagnostics

9.2.1 Accumulator and Debuncher Diagnostics

9.2.2 Targeting and Beam Transfer Diagnostics

\subsection{Controls}

10. Options for Future Improvements

10.1 Momentum Cooling in the Debuncher

10.2 Target Development

10.3 Improvements in Stochastic Cooling

10.4 Electron Cooling of the Core

10.5 The Overpass - A Main Ring Bypass

10.6 Low-Beta Improvements 


\section{INTRODUCTION AND OVERVIEW}

This report describes the design of the Tevatron I Antiproton Source, which will enable Fermilab to produce proton-antiproton collisions in the Tevatron accelerator. Center-of-mass energies near $2 \mathrm{TeV}$, by far the highest avallable anywhere in the world for high-energy physics research until at least the decade of the 1990's, will provide enormous opportunities for exclting new physics.

After the energy, the most important parameter determining the utility of a colliding-beam facility is the luminosity, or interaction rate per unit cross section. The first goal of the Tevatron I profect is to achieve a peak luminosity of $10^{30} \mathrm{~cm}^{-2} \mathrm{sec}^{-1}$ for proton-antiproton collisions at the maximum energy in the Tevatron.

The luminosity depends on the intensity and phase space density of the interacting beams. The design luminosity of $10^{30} \mathrm{~cm}^{-2} \mathrm{sec}^{-1} \mathrm{can}$ be achieved with as few as $1.8 \times 10^{11}$ protons and $1.8 \times 10^{11}$ antiprotons of appropriate phase space density. The number and phase-space density of antiprotons produced by bombarding a dense target with one pulse of protons from the Main Ring are too small by orders of magnitude to achieve the design luminosity. Thus $1 \mathrm{t}$ is necessary to collect many pulses of antiprotons in an accumulator ring and to increase thelr phase-space density, 1 . e. to cool them, by roughly six orders of magnitude. To minimize user frustration and maximize the average luminosity, the accumulation time should be as short as possible, at least short compared to the luminosity lifetime, which is expected to be larger than thirty hours. The second goal of the project is to accumulate and cool the required number of antiprotons in five hours or less, starting with no antiprotons in the Accumulator.

The design presented here to meet these goals is based on the method of stochastic cooling developed by van der Meer, Thorndahl, and coworkers. 1 This method generates a non-uniform phase-space density distribution of the accumulated antiprotons, with only the high-density core useful for colliding beams. Thus the source has been designed to accumulate a total of $4.3 \times 10^{11}$ antiprotons in 4 hours, of which typically $1.8 \times 10^{11}$ antiprotons from the high-density core will be infected into the Tevatron. Subsequent accumulation cycles starting with the antiprotons left from the previous cycle will require considerably shorter times to achieve the necessary core density.

The amount of cooling to be done depends on the phase-space density at production. The higher the initial density, the easier it is to achieve the final density. The yield of antiprotons per incident proton is proportional to the product of the spatial solid angle and the momentum spread accepted by the beam-transport system, and the initial phase-space density can therefore be increased only by decreasing the spot size and time spread of the antiprotons. The initial protons that produce the antiprotons determine these parameters and it is therefore useful to reduce the proton spot size and time spread. 
The proton rms spot size will be reduced to $0.38 \mathrm{~mm}$ by the use of standard quadrupole lenses. Further reduction would provide little gain because the apparent spot size is ultimately dominated by the large antiproton beam divergence and the finite length of the target. ${ }^{2}$ The first collection lens must match the large angular divergence of the antiproton beam at the target into the small angular admittance that is characteristic of a beam-transport system or a storage ring. This is achleved by using a Ilthium lens of the type developed by the Institute of Nuclear Physics at Novosibirsk.

The time spread can be minimized by $r$ manipulation of the proton beam in the Main Ring just prior to extraction. The narrow time spread and large energy spread of the resulting antiproton bunches can be transformed into bunches with a much lower energy spread by $r$ phase rotation in a separate $\mathrm{ring}$ called the Debuncher. The $\mathrm{rf}$ phase-rotation system ${ }^{4}$ makes it possible to start with a large momentum spread from the target, thereby increasing the antiproton flux. The reduced energy spread also greatly simplifies the design of the magnets and cooling systems of the Accumulator ring.

The design thus uses two fixed energy rings, the Debuncher and the Accumulator, located south of the Booster as shown in Fig. 1-1. The Accumulator has the same circumference as the Booster; the Debuncher is slightly larger. Both rings operate at a kinetic energy of $8 \mathrm{GeV}$, the Booster energy. The sequence of operations leading to colliding beams involves seven steps:

1. Proton Acceleration for Antiproton Production. Every two seconds, one Booster batch containing $3 \times 10^{\text {T2 }}$ protons in $80 \mathrm{rf}$ bunches is accelerated in the Main Ring to $125 \mathrm{GeV}$ and held at that energy while the $r$ manipulation described in the next step is carried out.

2. Preparation of Protons for Targeting. The 53-MHz $r$ voltage is lowered to cause each proton bunch to spread in time to 9 nsec. The rf voltage is suddenly increased to $4 \mathrm{MV}$ per turn and each proton bunch rotates by $90^{\circ}$ in phase space in $1.3 \mathrm{msec}$ to a short bunch of less than a nanosecond in width, but with an energy spread of about 0.4\%. This train of short bunches is extracted from the Main Ring at F17 as soon as the bunch rotation is completed.

3. Antiproton Production and Transport. The short proton bunches strike a tungsten target, producing a train of 80 equally short antiproton bunches. The peak energy deposition in the target is the same as that used successfully at CERN. $1.1 \times 10^{8} 8.9 \mathrm{GeV} / \mathrm{c}$ antiprotons are collected by the lithium lens and transported to the Debuncher. The momentum spread of the beam is $3 \%$ and the transverse beam emittances are $20 \pi \mathrm{mm}$-mrad in each plane.

4. Bunch Rotation in the Debuncher. The antiprotons are injected into 53-MHz $r f$ buckets in the Debuncher. The rf voltage is large enough 
that the antiproton bunches rotate just as the proton bunches rotated in the previous step. After a quarter of a synchrotron oscillation, the narrow time structure and large momentum spread have been transformed into a small momentum spread and a broad time structure. The $r f$ voltage is then rapidly lowered to match the bucket to the rotated bunch, and finally adiabatically lowered to reduce the momentum spread to $0.2 \%$.

5. Transverse Cooling in the Debuncher. After the $r$ manipulations, the horlzontal and vertical transverse emittances are stochastically cooled in the Debuncher from $20 \pi \mathrm{mm}-\mathrm{mrad}$ to $7 \pi \mathrm{mm}-\mathrm{mrad}$ during the almost two seconds before the next antiprotons are to be injected.

6. Antiproton Accumulation and Cooling. The antiprotons are extracted from the Debuncher and injected into the Accumulator. Successive batches are accumulated by rf stacking each batch at the edge of the stack. Between infection cycles, the stack is stochastically cooled using a combination of longitudinal and transverse cooling systems similar to the types developed by CERN for the AA ring. 5 A new batch of antiprotons with a density of about 7 antiprotons per eV is deposited at the stack tall every $2 \mathrm{sec}$. The fresh batch is moved by the coherent force of the stochastic-cooling system away from the injection channel and toward the center of the stack. The strength of the coherent force diminishes exponentially as the particles move away from the edge of the tail, causing the particle density to increase. Diffusion forces resulting from the Schottky noise of the antiproton stack and the thermal noise of the amplifiers cause the antiprotons to migrate from the high-density region toward the low-density region. As long as the coherent force is greater than the diffusion forces, the stack builds up in intensity until it reaches the core region where the coherent force is zero. Some antiprotons are lost during transfer and re stacking and some diffuse away from the stack into the chamber walls. Allowing for losses, $6 \times 10^{7}$ antiprotons are stacked in each pulse. In 4 hours, the core will grow to a density of $1.0 \times 10^{5}$ antiprotons per eV. The total number of antiprotons in the core will be $4.3 \times 10^{11}$. During this time the transverse cooling systems will have reduced the horizontal and vertical enittances to $2 \pi \mathrm{mm}-\mathrm{mrad}$.

7. Filling the Tevatron. After accumulation is complete, antiproton bunches of the desired intensity are individually extracted from the core, transferred to the Main Ring, accelerated to $150 \mathrm{GeV}$ and infected into the Tevatron. The same number of proton bunches of similar intensity are prepared in the Main Ring and injected into the Tevatron. Whether it is better to inject the protons or antiprotons first will be determined empirically. Both beams are then simultaneously accelerated to the desired energy.

Sufficient antiprotons for a luminosity of $10^{30} \mathrm{~cm}^{-2} \mathrm{sec}^{-1}$ can be produced in 4 hours by this sequence, even with reasonable losses in production, cooling, and beam transfer. The design luminosity can be achieved without 
exceeding a beam-beam tune shift of 0.0018 per crossing. As the Tevatron and the Antiproton Source become more reliable, longer collection times will become practical, resulting in higher luminosity.

Beam accumulation techniques are developing rapidly and it seems highly advisable to design an antiproton source that can accommodate future improvements. Accordingly, the third goal of this design is to incorporate flexibility for future improvements so that the Antiproton Source may ultimately achieve a luminosity of $10^{31} \mathrm{~cm}^{-2} \mathrm{sec}^{-1}$. The potential for luminosity of the proposed source is exhibited in Table 1-I, which shows the relationship between the number of accumulated antiprotons and the luminosity.

The peak luminosity and accumulation rate are limited not by the antiproton production rate but by the cooling systems of the Accumulator Ring. Higher luminosities may be achieved through improvements in these cooling systems. The present design uses less than a half of the total number of particles collected in each accumulation cycle to reach its design Iuminosity of $10^{30} \mathrm{~cm}^{-2} \mathrm{sec}^{-1}$. If future improvements can increase the final density by a factor of three, it will be possible to approach a luminosity of $10^{31} \mathrm{~cm}^{-2} \mathrm{sec}^{-1}$. The design of the rings therefore includes provisions (aperture and straight-section space) for:

(i) Momentum precooling in the Debuncher,

(11) Improved stochastic cooling in the Accumulator,

(1ii) Intermediate energy electron cooling in the Accumulator.

Other future improvements may include:

(iv) Target development,

(v) A Main Ring beam overpass at BO to allow antiproton accumulation to proceed in parallel with colliding beams in the Tevatron,

(vi) A superquadrupole to reduce beam size in the interaction region.

These features are not part of the initial design because it is difficult to foresee which improvements will be most feasible and cost-effective. The most beneficial cholces will be clear only after some experience in operating the collider.

The design of each system and some possible future improvements are described in greater detail in the following sections. 
Table 1-I. LUMINOSITY PROGRESSION

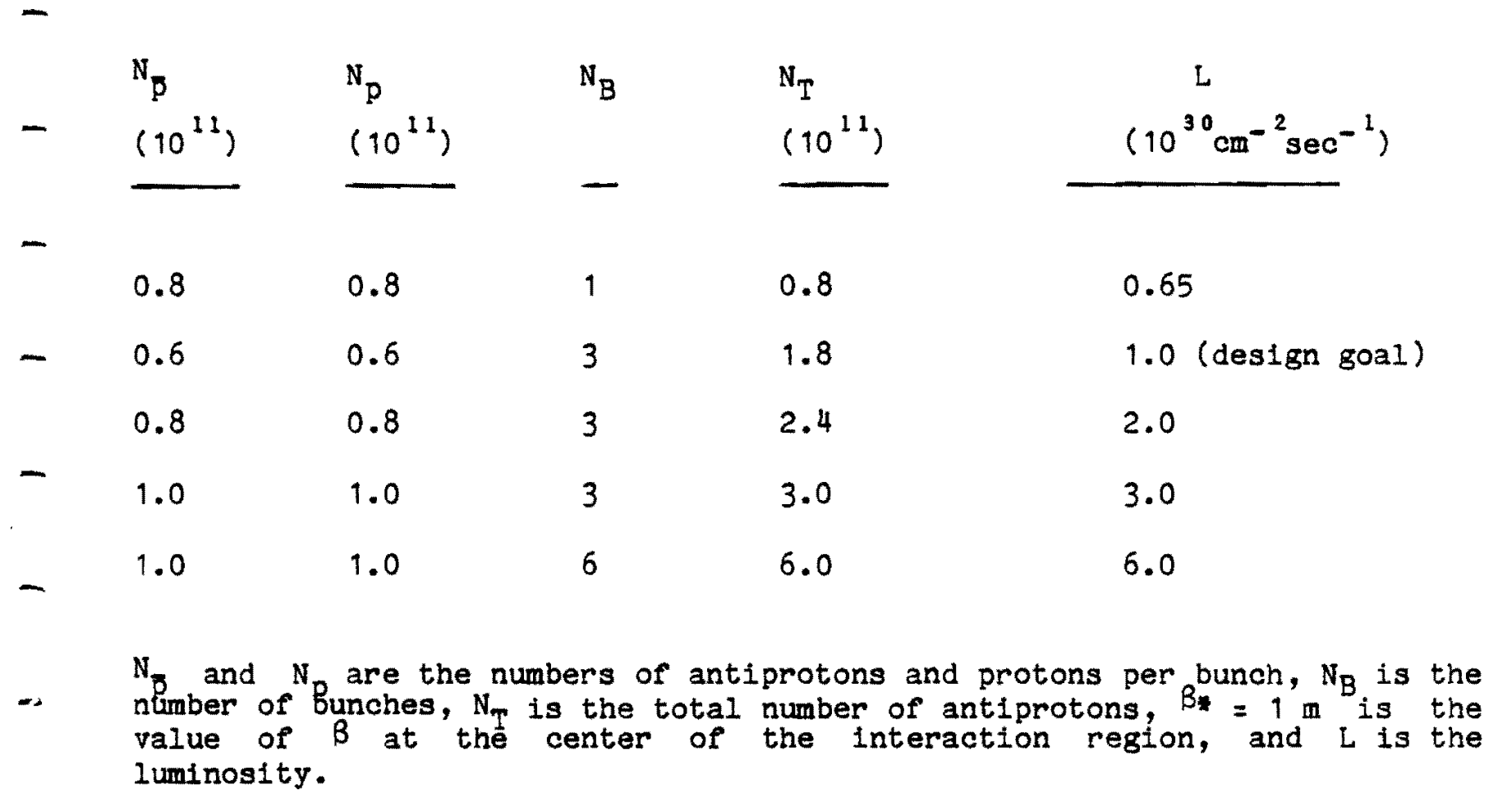




\section{References}

1. S. van der Meer, Stochastic Damping of Betatron Oscillations in the ISR CERN/ISR-PO/72-31 (1972) (unpublished).

D. Mohl, G. Petrucci, L. Thorndahl, and S. van der Meer, Physics Reports 58, p. 73 (1980).

2. C. Hojvat and A. Van Ginneken, Fermilab $\bar{p}$ Note 139 , July 16, 1981 (unpublished).

3. B. F. Bayanov et al., Nucl. Inst. Methods 190, 9 (1981).

4. A. G. Ruggiero, Fermilab D̄ Note 102, January, 1981 (unpublished).

5. S. van der Meer, Tochastic Stacking in the Antiproton Accumulator CERN/PS/AA/78-22 (1978) (unpublished). 


\section{PROTON ACCELERATION}

The accumulation cycle begins by accelerating a single Booster batch of protons in the Main Ring to $125 \mathrm{GeV}$ with the existing rf system. This Booster batch consists of a string of 80 bunches spaced by $5.6 \mathrm{~m}$; its total length is $445 \mathrm{~m}$. A single batch fills less than one-thirteenth of the Main Ring cirqumference. The booster intensity record for a single batch is $3.4 \times 10^{12}$. For the purpose of this report, it is assumed that an intensity of $3.0 \times 10^{12}$ protons per Booster batch will be standard operating intensity for p production. The minimum time needed to accelerate a single batch to $125 \mathrm{GeV}$ in the Main Ring can be reduced to 1.87 seconds. A flat-top of less than $0.13 \mathrm{sec}$ is added to provide time for the $r f$ beam manipulation before extraction. The cycle time is 2 seconds. The major beam properties are given in Table 2-I.

A reasonable estimate for the normalized betatron emittance, based on measurements, is $24 \pi \mathrm{mm}$-mrad. This value includes $95 \%$ of the beam. If $\sigma_{H, V}$ denotes the rms beam size and $B_{H}$, the lattice amplitude function, the emittance can be expressed in terms of these quantities as

$$
\varepsilon_{H, V}=6^{\pi} \frac{\sigma_{H, V}^{2}}{\beta_{H, V}}
$$

The longitudinal phase-space area $S$ of individual bunches in a booster batch has been measured to be $0.3 \mathrm{eV}-\mathrm{sec}$ or less. ${ }^{2}$ If the particle distribution is biGaussian in these variables, this value includes 95\% of the beam. There is reason to belleve this number could be reduced to $0.2 \mathrm{eV}-\mathrm{sec}$ in the future when improvements are made to the Main RIng and the Booster rf systems.

For energies well above the transition energy, a bunch area $S$ half as large as the bucket area, and a bunch shape matched to the rf bucket, the rms bunch length $\sigma_{e}$ and rms momentum spread $\sigma_{p} / p$ are

$$
\sigma_{e}=(142 \mathrm{~cm}) \sqrt{\frac{S}{\sqrt{v E}}} \quad \sigma_{p / p}=\left(0.0112 \sqrt{\frac{S}{\sqrt{V^{3} / V}}} .\right.
$$

The bunch area can be expressed in terms of these quantities as

$$
S=6 \pi \beta_{E} \frac{\sigma_{e}}{c} \frac{\sigma_{p}}{p} .
$$


The bucket area of a stationary bucket $B$ and the phase oscillation period $\mathrm{T}_{\mathbf{S}}$ are respectively

$$
B=(0.34 \mathrm{eV}-\mathrm{sec}) \quad \sqrt{\mathrm{VE}} \quad \mathrm{T}_{\mathrm{S}}=(1 \mathrm{msec}) \sqrt{\mathrm{E} / \mathrm{V}} .
$$

In all these equations $E$ is in GeV, $V$ in $M V$ and $S$ in $e V-s e c$.

For a fixed antiproton momentum spread, the bunch area of the antiprotons is minimized by making the time spread of the extracted proton bunches as narrow as possible. At the end of acceleration when the $r f$ voltage is $4 \mathrm{MV}$ the relevant beam parameters are $T_{s}=5.6 \mathrm{msec}, \sigma_{e}=16 \mathrm{~cm}$, $\sigma_{p} / p=2.4 \times 10^{-4}$.

The rf voltage is slowly reduced from $4 \mathrm{MV}$ to $30 \mathrm{kV}$ so that the bunch motion remains matched to the $r f$ bucket shape. At $30 \mathrm{kV}$, the beam parameters are $T_{s}=65 \mathrm{msec}, \sigma_{\mathrm{e}}=56 \mathrm{~cm}$, and $\sigma_{\mathrm{p}} / \mathrm{p}=6.8 \mathrm{x} 10^{-5}$. The adiabatic reduction of the $r f$ voltage is accominplished in $100 \mathrm{msec}$. At that moment the synchrotron phase of the bunch extends to $\pm 90^{\circ}$.

The $\mathrm{rf}$ voltage is then raised abruptly to $4 \mathrm{MV}$ within two revolutions or $42 \mathrm{Hsec}$. The bunches are no longer matched to the buckets, so they start to rotate as in Fig 2-1. After a quarter of a phase oscillation or 1.4 msec, the time spread of the beam has been reduced by more than an order of magnitude. At that moment the beam parameters are $T_{s}=5.6 \mathrm{msec}, \sigma_{e}=5.0$ $\mathrm{cm}$, and $\sigma_{\mathrm{p}} / \mathrm{p}=8.4 \times 10^{-4}$. After these operations are completed, a momentum spread of $0.4 \%$ contains $95 \%$ of the beam.

Measurements made in the Main $\mathrm{Ring}^{2}$ have shown that the available momentum aperture is between $0.5 \%$ and $1 \%$ at $100 \mathrm{GeV}$. The loss of beam due to the $0.4 \%$ momentum spread is expected to be small, since very little of the beam will extend 1nto the bad-field region of the aperture. As soon as the bunch rotation is complete, all 80 bunches are ejected into the 125-GeV transport line at F17.

\section{References}

1. C. Moore et al., Fermilab EXP Note 101, February 1980 (unpublished).

2. J. Griffin (unpublished). 
Table 2-I. MAIN RING BEAM PARAMETERS

Proton Beam Kinetic Energy e Extraction Relativistic Factors: $B$

$$
\gamma
$$

$B \rho$, magnetic rigidity Momentum, P

Number of Booster batches accelerated

Number of Proton Bunches

Total number of protons per Batch

Main Ring Cycle Time

Betatron Emittance, $95 \%$ of beam, ( $H$ and $V$ )

Longitudinal Emittance, $95 \%$ of beam

RF harmonic number ( $h$ )

RF Frequency $125 \mathrm{GeV}$

Revolution Period e $125 \mathrm{GeV}$

Booster Batch Time Length

Transition Energy $\left(\gamma_{t}\right)$

Betatron tune number ( $H$ and $V$ )

$\eta=r^{2}-\gamma_{t}-2$

Maximum RF voltage

Average Radius
$125 \mathrm{GeV}$

0.99997

134.2253

$4200.73 \mathrm{kG}-\mathrm{m}$

$125.93473 \mathrm{GeV} / \mathrm{C}$

one

80

$3.0 \times 10^{12}$

$2.0 \mathrm{sec}$

$0.2 \pi \mathrm{rm}$

$0.3 \mathrm{eV}-\mathrm{sec}$

1113

53. $1021 \mathrm{Mhz}$

20.96 usec

1.51 usec

18.75

19.4

$-0.0028$

4.0 MV

$1000 \mathrm{~m}$ 


\section{TARGETING AND TRANSPORT}

\subsection{Main Ring Extraction}

Extraction of $125-\mathrm{GeV}$ protons from the Main Ring for the production of antiprotons takes place at location F17. Two Lambertson magnets deflect the extracted beam vertically by $32.8 \mathrm{mrad}$ into the transport line, which is located just above the Main Ring magnets. The geometry and expected beam sizes at the Lambertsons are shown in Fig. 3-1a and 3-1b. The horizontal beam size at $125 \mathrm{GeV}$ includes the contribution of the $0.4 \%$ momentum spread of the tightly bunched proton beam. The large horizontal size of the bunched beam uses most of the available Main Ring aperture to the inside of the Ring at the extraction point. As seen in Fig. 3-1, the septum of each Lambertson will be located well within the Main Ring aperture, preventing parasitic extraction at F17 during the 400-GeV slow-extraction HEP program. The Lambertsons will be withdrawn from the aperture during 400-GeV operation. 3-I.

The relevant parameters of the Main Ring lattice are listed in Table

TABLE 3-I. PROPERTIES OF SELECTED MAIN RING MINISTRAIGHTS

Horizontal-Plane Lattice Functions

Location

C48 Ricker

F12

F13

F14

F15 (Bump 1)

F16

F17 (Bump 2)

(Extraction)

F18 (Bump 3)

F19

F21

F22 (Bump 4)

F23

F24

F25

F25

$$
B
$$

(m)

$\alpha$

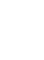

Phase

Relative

to F17

(Modulo

$360^{\circ}$ )

$\begin{array}{lr}102.414 & 0.46696 \\ 29.601 & -0.5731 \\ 95.356 & 1.8584 \\ 28.383 & -0.5893 \\ 97.247 & 1.8396 \\ 30.093 & -0.6239 \\ & \\ 99.648 & 1.9388 \\ 28.865 & -0.5582 \\ 94.322 & 1.8156 \\ 28.912 & -0.6177 \\ 99.541 & 1.8926 \\ 30.073 & -0.5983 \\ 97.400 & 1.9056 \\ 28.365 & -0.5666 \\ 95.244 & 1.8100\end{array}$

$\begin{array}{rc}-90.11^{\circ} & \text { (Existing) } \\ -168.30^{\circ} & 0.0 \\ -135.74^{\circ} & 0.0 \\ -99.29^{\circ} & 34.0 \\ -66.32^{\circ} & 42.5 \\ -31.47^{\circ} & 42.0 \\ & \\ 0.00^{\circ} & 32.0 \\ 35.20^{\circ} & 52.0 \\ 68.42^{\circ} & 0.0 \\ 104.71^{\circ} & 28.0 \\ 136.92^{\circ} & 27.5 \\ 171.32^{\circ} & 35.0 \\ 203.21^{\circ} & 0.0 \\ 239.23^{\circ} & 35.0 \\ 272.60^{\circ} & 43.5\end{array}$

Space

Avallable

(in.)

43.5 
The kicker at location $\mathrm{C} 48$ causes a 43-mm horizontal displacement at F17, which is sufficient to cross the Lambertson septum. The required angular deflection at 048 is obtained with a voltage of $60.0 \mathrm{kV}$, well within the present operating range of the supply. Given the Main Ring lattice characteristics, the klck at $\mathrm{C}-48$ results in an angular displacement of $+0.847 \mathrm{mrad}$ at $F 17$ as well as a horizontal displacement.

A local four-magnet orbit bump at F17 is used to position the closed orbit during 8-GeV injection in the center of the restricted aperture at F17 and to move the orbit close to the Lambertson septa during 125-GeV extraction. Four standard 35-in. long bump magnets are placed at F15, F17, F18 and F22. The closed orbit at $125 \mathrm{GeV}$ can be adjusted over a range of $\pm 37 \mathrm{~mm}$ at location F17.

Vertical extraction was chosen since it allows the extracted proton beam to clear the Main Ring magnet, F17-1, just downstream of the Lambertsons with a minimum deflection. The return coll on the upstream end of F17-1 has been modified to pemit the beam pipe containing the extracted $125-\mathrm{GeV}$ to be as close to the magnet steel as possible. The length of the medium straight section at F17 limits the maximum energy that can be extracted to $125 \mathrm{GeV}$.

The Lambertson magnets are $204 \mathrm{in.}$ long and are excited to between 12 and $13 \mathrm{kG}$ at $125 \mathrm{GeV}$. The second Lambertson accommodates a vertical beam translation of $5 \mathrm{in}$.

\subsection{Proton Transport}

The initial step in developing a 125-GeV extracted beam at $\$ 17$ was to redesign the existing $80-\mathrm{GeV}$ line, which extends for 1000 feet from $F 17$ to F25 in the Main Ring Tunnel, by adding magnets. Although this design satisfied the requirements of the project, its estimated power consumption of $2 \mathrm{MW}$ would place a heavy burden on the operating cost of the Antiproton Source. The elements of the present line are placed immediately above the Main Ring magnets, which are in turn just above the magnets. As a consequence, difficult operational problems are encountered whenever work must be performed on the Main Ring or the Tevatron. In particular, it has already been necessary to dismantle the extracted beam in these locations. In order to eliminate these problems, a new beam line was designed.

The design of the new 125-GeV proton transport line was based on the following requirements:

(i) It must leave the Main Ring tunnel as close as possible to location $\mathrm{F} 18$.

(11) The target elevation is to be $2.1 \mathrm{~m}$ above Main Ring beam height. 
(1ii) It must transport a $125-\mathrm{GeV}$ proton beam with a momentum spread of $\pm 0.2 \%$.

(iv) It must transport the $8.9-\mathrm{GeV} / \mathrm{c}$ cooled antiproton beam with an emittance of $2.0 \pi \mathrm{mm}-\mathrm{mrad}$.

(v) It must be possible to bypass the target and collection system in the reverse direction.

(vi) It must produce a round proton beam spot at the target which can be varied from $0.18 \mathrm{~mm}$ to $0.75 \mathrm{~mm} \mathrm{rms}$.

(vii) It must have zero dispersion at the target.

(viii) The power consumption must remain below $500 \mathrm{~kW}$.

A layout of the beam is shown in Fig. 3-2 and its parameters are tabulated in Table 3-II. It consists of 10 EPB dipoles excited to a field of $13 \mathrm{kG}$ or less and 1432120 quadrupoles excited to a field gradient of $4.75 \mathrm{kG} / \mathrm{in}$. or less. Three of the dipoles, indicated by ( $R$ ), are rotated by $45^{\circ}$ to achieve the necessary vertical and horizontal bends with the minimum number of magnets. The Main Ring Tunnel must be widened over a distance of roughly $18 \mathrm{~m}$ in order to accommodate the four EPB dipoles and one 3Q120. The length of the beam is $174 \mathrm{~m}$, of which $41 \mathrm{~m}$ are located in the Main Ring Tunnel. The remainder of the beam elements are located in $a$ new 115-m long enclosure. The new enclosure and the Main Ring Tunnel are connected by an 18-m long pipe.

A $4.5^{\circ}$ bending magnet, which bends the reverse antiproton beam to the left, is placed within the final focus section between Q2B and Q3A in order to allow the target and antiproton collection lens to be bypassed whenever cooled antiprotons are transported back to $F 17$. The total power consumption of the beam is estimated to be just below $500 \mathrm{~kW}$, when the quadrupoles are adjusted to make the rms beam size at the target to be $0.378 \mathrm{~mm}$. In Table 3-III the gradients of the four final focusing quadrupoles, which obtain the required range of beam sizes, are listed. 
Table 3-II. 125-GEV TRANSPORT ELEMENTS

\begin{tabular}{|c|c|c|c|c|c|}
\hline NAME & $\begin{array}{l}\text { DIST. TO } \\
\mathrm{F} 17(\mathrm{~m})\end{array}$ & TYPE & $\begin{array}{l}\text { FIELD } \\
(\mathrm{T})\end{array}$ & $\begin{array}{l}\text { GRADIENT } \\
(\mathrm{T} / \mathrm{m})\end{array}$ & FUNCTION \\
\hline $\begin{array}{l}\text { LAM1 } \\
\text { LAM2 }\end{array}$ & $\begin{array}{l}2.74 \\
8.38\end{array}$ & $\begin{array}{l}\text { Lamberston } \\
\text { Lambertson }\end{array}$ & $\begin{array}{l}1 \cdot 32 \\
1 \cdot 32\end{array}$ & & $\begin{array}{l}\text { Bend up } \\
\text { Bend up }\end{array}$ \\
\hline HBL 1 & 21.7 & EPB & 1.314 & & Bend Left \\
\hline HBL 2 & 25.0 & EPB & 1.314 & & Bend Left \\
\hline RHBLL 3 & 28.4 & EPB (R) & 1.314 & & Bend Left/Down \\
\hline RHBL 4 & 31.7 & EPB (R) & 1.314 & & Bend Left/Down \\
\hline & $\begin{array}{l}35.1 \\
64.2\end{array}$ & $\begin{array}{l}30120(60) \\
30120(60)\end{array}$ & & $\begin{array}{l}6.8(13.6) \\
5.0(10.0)\end{array}$ & $\begin{array}{l}\text { Vertical Focusing } \\
\text { Vertical Focusing }\end{array}$ \\
\hline RHBR 1 & 67.5 & EPB (R) & 1.314 & & Bend Right/Down \\
\hline HBR2 & 70.9 & $\mathrm{EPB}$ & 1.314 & & Bend Right \\
\hline HBR3 & 74.2 & EPB & 1.314 & & Bend Right \\
\hline HBR 4 & 77.6 & EPB & 1.314 & & Bend Right \\
\hline & $\begin{array}{l}80.9 \\
94.1\end{array}$ & $\begin{array}{l}3 Q 120 \\
3 Q 120\end{array}$ & & $\begin{array}{l}12.50 \\
16.44\end{array}$ & $\begin{array}{l}\text { Horiz. Focusing } \\
\text { Vertical Focusing }\end{array}$ \\
\hline Q5A & $107 \cdot 3$ & $3 Q 120$ & & 18.00 & Horiz. Focusing \\
\hline Q5B & 110.7 & 32120 & & 18.00 & Horiz. Focusing \\
\hline VB1 & 123.7 & $\mathrm{EPB}$ & 0.85 & & Bend Down \\
\hline VB2 & 127.0 & EPB & 0.85 & & Bend Down \\
\hline FQ1A & 130.7 & 30120 & & 11.81 & Vertical Focusing \\
\hline FQ1B & 134.1 & $3 Q 120$ & & 11.81 & Vertical Focusing \\
\hline FQ2A & 139.6 & $3 Q 120$ & & 10.62 & Horlz. Focusing \\
\hline FQ2B & 143.0 & 32120 & & 10.62 & Horiz. Focusing \\
\hline FQ3A & 154.9 & $3 Q 120$ & & 12.79 & Vertical Focusing \\
\hline FQ3B & 158.3 & 30120 & & 12.79 & Vertical Focusing \\
\hline FQ4A & 163.9 & $3 Q 120$ & & 9.58 & Horiz. Focusing \\
\hline FQ4B & 167.2 & $3 Q 120$ & & 9.58 & Horiz. Focusing \\
\hline TARGET & 174.1 & & & & \\
\hline
\end{tabular}

Target Location on Site Coordinates

$$
\begin{aligned}
& x=586.0 \mathrm{ft} \\
& y=-2064.3 \mathrm{ft} \\
& z=728.75
\end{aligned}
$$


Table 3-III. GRADIENT OF FINAL FOCUSING QUADRUPOLES

AS A FUNCTION OF BEAM SIZE AT THE TARGET, IN $\mathrm{T} / \mathrm{m}$.

(MAXIMUM GRADIENT $18 \mathrm{~T} / \mathrm{m}$ )

$\begin{array}{llllr}\sigma(\mathrm{mm}) & F Q 1 / 1 A & \text { FQ2/2A } & \text { FQ3/3A } & \text { FQ4/4 } \\ 0.188 & -14.29 & 10.76 & -12.57 & 13.70 \\ 0.375 & -11.81 & 10.62 & -12.80 & 9.58 \\ 0.75 & -10.74 & 10.07 & -10.22 & 2.04\end{array}$




\subsection{Targeting And Antiproton Production}

In order to estimate and optimize the number of antiprotons produced, three different topics need to be discussed as follows:

1. details of the production cross sections obtained from existing experimental data;

2. the choice of primary proton energy, antiproton momentum, antiproton longitudinal acceptance and antiproton transverse acceptance; and

3. limitations introduced by targeting and the antiproton collection system.

3.3.1 Production Cross Sections. The available data on the cross sections for the production of antiprotons have been described by a phenomenological formula that includes the dependence on the incident proton energy, the scaling variable $x_{p}$ and the target nucleus. The yield of antiprotons from a tungsten target which is collected in a solid angle defined by a laboratory polar angle of $60 \mathrm{mrad}$ for proton energies of interest at Fermilab was calculated using this formula and the results are shown in Fig. 3-3. It can be seen that there is a plateau at $120 \mathrm{GeV}$ for the production of antiprotons between 8 and $13 \mathrm{GeV} / \mathrm{c}$.

3.3.2 Proton Energy. The yield of antiprotons per unit volume of phase space per unit time changes very slowly with proton energy above $150 \mathrm{GeV}$ when the Main Ring cycle time is taken into consideration. Although small gains in yield could be obtained by going to a higher energy, a proton energy of $125 \mathrm{GeV}$ was chosen because it is the maximum energy that can be extracted from a medium straight section such as F17. F17 provides a very convenient location for the Antiproton Source because it is near the Booster and the main accelerator work area. The cholce was also influenced by the rapid increase in operating cost as the energy of the Main Ring is increased above $125 \mathrm{GeV}$. This energy is compatible with the Colliding Beams Detector overpass. The overpass, which is described in Sec. 10.5, limits peak energy of the Main Ring to $200 \mathrm{GeV}$.

3.3.3 Antiproton Momentum. The optimum antiproton momentum is $10.0 \mathrm{GeV} / \mathrm{c}$ for an incident proton energy of $120 \mathrm{GeV}$. The yield is $90 \%$ of the optimum yield throughout the range from $7.5 \mathrm{GeV} / \mathrm{c}$ to $13.0 \mathrm{GeV} / \mathrm{c}$. Although stochastic cooling can be accomplished throughout this momentum range, the desire to keep power consumption low favors the lower momentum. Since the normal injection momentum of the Main Ring, $8.89 \mathrm{GeV} / \mathrm{C}$, is within this range of momenta, it is a reasonable choice. It has the advantage of permitting the transfer of antiprotons directly from the Accumulator to the Main Ring. In addition, it opens the possibility of utilizing protons directly from the Booster as an alternative source of particles during commissioning of the source. 
3.3.4 Antiproton Longitudinal Acceptance. Stochastic cooling performance depends critically on the flux of $\bar{p}^{\prime} s$ injected in the Accumulator and on the particle density per unit of energy spread. This density, which is inversely proportional to the longitudinal emittance of the antiprotons, is determined in part by the time spread of the $\bar{p}$ 's at production, as discussed in Chapter 2.

The Debuncher can debunch a total momentum spread of $3 \%$ into a momentum spread which can be accommodated by the Accumulator. Although a larger momentum spread will result in a larger flux the Accumulator is not able to cool the larger but lower density flux. The $\bar{p}$ collection and transport system are designed to accept $8.4 \%$ FWHM momentum spread. Initially the momentum acceptance will be Ilmited by collimation to $3 \%$.

3.3.5 Antiproton Transverse Acceptance. The selection of parameters described above permits the calculation of expected antiproton yields versus transverse acceptance.

The calculations depend crucially on the collection system downstream of the target. A comparison of different collection systems has been performed ${ }^{2}$, taking into account the large momentum spread of the antiproton beam. The advantages of a device such as the lithium lens which was developed at the INP in Novosibirsk are clear. It has a very short focal length and it focuses in both transverse planes. The parameters of the Iithium lens collector were chosen as follows: Radius $=1 \mathrm{~cm}$, Gradient $=$ $1000 \mathrm{~T} / \mathrm{m}$, Length $=15 \mathrm{~cm}$. Within the present technology developed at Novosibirsk a repetition rate of $1 \mathrm{~Hz}$ is feasible. The lens has a focal distance of $14.5 \mathrm{~cm}$. The short focal distance requires the use of a dense target material with a short nuclear absorption length.

Antiprotons yields have been calculated with a Monte Carlo program ${ }^{1}$ that includes the phenomenological description of the production cross section, the development of hadronic showers in the target and $\bar{p}$ production by secondaries, multiple scattering and absorption. The result of these calculations is shown in F18. 3-4 for two different rms proton beam spot sizes, $\sigma_{x}=\sigma_{y}=0.038 \mathrm{~cm}$ and $\sigma_{x}=\sigma_{y}=0.022 \mathrm{~cm}$. The number of antiprotons increases approximately linearly with the transverse acceptance above $20 \pi \mathrm{mm}$-mrad for $\sigma=0.038 \mathrm{~cm}$. For smaller beam size, the departure from linearity above $20 \pi \mathrm{mm}$-mrad lithium lens radius and its gradient. The optimum target length is $5 \mathrm{~cm}$.

3.3.6 Targeting Limitations. The energy density deposited by a $125-\mathrm{GeV}$ proton beam in tungsten has been calculated using the program MAXIM ${ }^{4}$. The maximum energy density deposited within a $5-\mathrm{cm}$ long target is shown in Fig. 3-5 versus the rms size of the proton beam, $\sigma$. Within the errors of the calculation, it varies as $\sigma^{-2}$.

The subject of high energy density deposition in targets has been the subject of a Fermilab Workshops. The general consensus reached at that 
workshop was that metals could sustain an energy-density deposition of up to 200 Joules $\mathrm{gm}^{-1}$ before the onset of shock waves that could result in the density depletion of the target. This figure is consistent with the operational experience of no failures of the CERN AA target. Calculations show that the target sustains a maximum energy density of $185 \mathrm{~J} / \mathrm{gm}$.

Using a maximum energy density of 200 Joules $\mathrm{gm}^{-1}$, we have calculated the maximum number of protons per pulse versus beam RMS size that can be targeted. The results are shown in Fig. 3-5.

The expected number of $\bar{p}$ per pulse as a function of beam size obtained from these calculations is shown in Table 3-IV; By choosing $\beta_{x}=B_{y}=3 \mathrm{~m}$ at the target and a proton intensity of $3 \times 10^{12}$ per Booster batch, the maximum energy density deposition in the target is of the order of 200 Joules $\mathrm{gm}^{-1}$.

A feasibility study was carried out ${ }^{6}$ on sweeping the proton beam across the target to decrease the energy density deposited in the material. The antiprotion acceptance must be simultaneously swept to track the proton beam spot. If the sweeping covers several proton beam diameters, it is possible to target $3 \times 10^{12}$ protons on beam spots corresponding to $\beta<3 \mathrm{~m}$, which will give an increased number of antiprotons accepted per proton. The design of the target area makes it possible to incorporate $a$ beam-sweeping system in the future.

Table 3-IV. NUMBER OF ANTIPROTONS PER PULSE

$\begin{array}{lllll}\beta(m) & \sigma_{x}=\sigma_{y}(\mathrm{~cm}) & \text { Np Max. } & \bar{p} / \mathrm{p} & \bar{p} / \mathrm{pulse} \\ 1.0 & 0.0218 & 1.0 \times 10^{12} & 6.8 \times 10^{-5} & 0.68 \times 10^{8} \\ 2.0 & 0.0309 & 2.0 \times 10^{12} & 5.7 \times 10^{-5} & 1.14 \times 10^{8} \\ 3.0 & 0.0378 & 3.0 \times 10^{12} & 4.9 \times 10^{-5} & 1.46 \times 10^{8}\end{array}$

Notes:

1. $\beta$ 1s for the proton beam at the center of the $\operatorname{target}\left(\beta_{x}=B_{y}=\right.$ 2. $\begin{aligned} & 3.0 \times 10^{12} \text { is less than the record Booster batch intensity of } \\ & 3.4 \times 10^{12} \text {. }\end{aligned}$

3. The yield in $\bar{p} /$ proton is for $4 \% \Delta p / p$ and $20 \pi$ mm-mrad.

3.3.7 The Lithium Lens Collector. Antiprotons diverging from the target are collected by a 15-cm long lithium lens of 1-cm radius. The basic principle of this device is that a uniformly distributed electric current in a cylindrical conductor produces an azimuthal magnetic fleld with a 
constant radial gradient. Thus, such a device will axially focus a particle beam passing along the axis of the conductor. The desired gradient of $1000 \mathrm{~T} / \mathrm{m}$ can be produced by a current pulse of $0.5 \mathrm{MA}$. Current uniformity is obtained by adjusting the pulse length to make the skin depth close to the radius. A $1-\mathrm{msec}$ full-width unipolar sine-like pulse is adequate. A 10-cm long lens capable of handling such pulses at a 2-Hz repetition rate has been designed at Novosibirsk. Its design will require some minor development work to meet our requirements. The lens is now under construction at the Institute for Nuclear Physics. The optical performance and the effect of beam on the service life will be made when protons are brought to the target station.

The program MAXIM has been used to calculate the energy deposition by a 125-GeV proton beam in a lithium as described above. For this simulation the protons strike a 5-cm long tungsten target located at the focus, $14.5 \mathrm{~cm}$ upstream of the lens. The energy deposition in the lens from secondary particles emerging from the target as well as the non-interacting protons is included in the calculation. The geometry includes the beryllium windows and titanium vessel which contains the lithium. The maximum energy deposited is $0.10 \mathrm{GeV} \mathrm{cm}$-3ncident proton for a beam size of $\sigma_{\mathrm{x}}=\sigma_{\mathrm{y}}=0.0378 \mathrm{~cm}$. The energy density deposited by the beam in a cyliñder $y$ of radius $0.04 \mathrm{~cm}$ concentric with the beam is $0.075 \mathrm{GeV}$ $\mathrm{cm}^{-3}$ /proton. Assuming $3.0 \times 10^{12}$ incident protons the average temperature rise in the inner core of the lens due to beam heating is $\Delta \mathrm{T} \sim 24^{\circ} \mathrm{C} / \mathrm{pulse}$.

Joule heating caused by the electrical current pulse results in a temperature rise of $70^{\circ} \mathrm{C} /$ pulse. If the temperature of the cooling water is $20^{\circ} \mathrm{C}$, the temperature at the core of the lens will rise to $114^{\circ} \mathrm{C}$ after each pulse. In $0.5 \mathrm{~Hz}$ operation there is adequate time between pulses for the lithium to return to $20^{\circ} \mathrm{C}$. At higher pulse rates, higher beam intensities, or smaller beam spots, the lithium could be heated locally to its melting point of $180^{\circ} \mathrm{C}$. The lenses are designed to withstand the mechanical strain resulting from the $1.5 \%$ increase in volume if melting occurs.

3.3.8 Alternative Collectors. A study has been made of the relative merits of $\bar{p}$ collection schemes that utilize a lithium lens, a pulsed quadrupole multiplet, or a 5036 triplet. It is assumed that the collector follows a source point of radius $0.4 \mathrm{~mm}$ and renders the divergent cone of $\bar{p}$ 's into a parallel beam. This beam is then passed through a four-quadrupole telescope and brought to debuncher injection conditions of $B_{x}=B_{y}=10 \mathrm{~m}$ and $\alpha_{x}=\alpha_{y}=0$.

The best collector should ideally put as many $\bar{p}$ as possible into the acceptance of the Debuncher. Chromatic aberrations causing effective emittance growth occur in all three collectors. Multiple scattering in the lithium lens also causes real enittance growth. We have studied the emittance growths for each scheme - generally for different initial emittances and different $\Delta p / p$. The program TURTLE has been used to obtain the fraction $f$ of started particles that are aberrated out of the initial ellipse phase areas and, more generally, to present the transmission $t$ ( $t=$ 
1 - f). The ray generation is uniform in $x-x^{\prime}$ and $y-y^{\prime}$ phase spaces and flat in $\Delta p / p$. Other effects such as depth of field due to target length or scattering within the target were not considered for this study.

First we consider the 5236 collector. An emittance of $5.0 \pi \mathrm{mm}$-mrad is assumed. This corresponds to a maximum source angle of 12.5 mrad. The typical butterfly shapes are generated in phase space for nonzero momentum spread and significant effective emittance growth is observed. The transmissions drop to $70 \%$ and $32.5 \%$ for $\Delta p / p= \pm 0.5 \%$ and $\pm 2.0 \%$, respectively. Since quadrupole apertures limit the maximum emittance to about $7.5 \pi \mathrm{mm}$-mrad for this system, larger emittance were not tried.

Next we systematically compare the lithium-lens and pulsed-quadrupole collectors for the three initial emittances $5 \pi, 10 \pi$, and $20 \pi \mathrm{mm}-\mathrm{mr}$. A momentum spread of $\pm 2 \%$ is assumed in all cases. The effects of multiple scattering were introduced in the lithium in 2.5-cm sections. Random deflections up to $\pm 2 \sigma$ were applied to $\bar{p}$ trajectories, where $\sigma$ is the rms multiple-scattering angle. The transmissions are collected into Table $3-V$, where we also list the results obtained with no multiple scattering in the lithium lens for comparison. The $\bar{p}$ yeilds quoted in Sec. 3.3.6 and Table 3-IV already include multiple scattering in the lithium lens.

The results of this analysis demonstrate the superiority of the lithium-lens collector, especially for large emittances and momentum spreads. Even pulsed 60-kG quadrupole devices caused severe emittance growth for large emittances (> $10 \pi \mathrm{mm}-\mathrm{mr}$ ).

Table 3-V. TRANSMISSION INTO

$\beta=10 \mathrm{~m}$ WAISTS FOR $\Delta \mathrm{p} / \mathrm{p}= \pm 2.0 \%$ RAYS FOR

DIFFERENT COLLECTORS

\begin{tabular}{ccccc}
$\begin{array}{c}\text { Emittance } \\
\text { (mm-mrad) }\end{array}$ & $\begin{array}{c}\text { Maximum } \\
\text { Angle } \\
\text { (mrad) }\end{array}$ & $\begin{array}{c}\text { Lithium } \\
\text { Lens }(\boldsymbol{\phi})\end{array}$ & $\begin{array}{c}\text { Pulsed } \\
\text { Quadrupoles ( })\end{array}$ & $\begin{array}{c}\text { Lithium Lens } \\
\text { (No Scattering\%) }\end{array}$ \\
\hline & & & & \\
$5 \pi$ & 12.5 & 65.9 & 60.6 & 89.6 \\
$10 \pi$ & 25.0 & 64.9 & 45.1 & 75.0 \\
$20 \pi$ & 50.0 & 59.8 & 30.3 & 74.8
\end{tabular}


Linear and nonlinear horns of the type developed for the CERN AA ring could be utillzed in case the lithlum lens development lags. To optimize the $\overline{\mathrm{p}}$ rate they require significantly longer targets. Preliminary results indicate that horns would not provide as good a collection efficiency as the proposed lithium lens at our $\bar{p}$ energy. The larger currents required for the $8.89 \mathrm{GeV} / \mathrm{c} \overline{\mathrm{p}} \mathrm{s}$, as compared with $3.5 \mathrm{GeV} / \mathrm{c}$ at CERN, may make the horn construction very difficult.

\subsection{Antiproton Target}

The yield of antiprotons into a given acceptance increases as the proton beam spot decreases until multiple scattering in the target material starts to contribute to beam-size growth. Smaller proton beam sizes will result in increasing energy density deposition in the target material. A compromise must be made between the brightness of the antiproton beam and the expected lifetime of the target itself.

3.4.1 Material Choice. It is obvious that the target must have a high density and high melting point. A compilation of mechanical properties for different materials was performed. A figure of merit to compare the mechanical properties was obtained from the yield stress divided by the coefflcient of thermal expansion and the modulus of elasticity. On this basis rhenium, tungsten and tungsten-rhenium alloys are in increasing order for this flgure of merit. The coefficient of heat conductivity could also be included in the figure of merit without signiflcantly altering the cholce of material.

The high-temperature behavior of tungsten-rhenium alloys shows considerable increase of yield stresses with respect to tungsten, but Ifttle change in the coefficient of thermal expansion or the modulus of elasticity.

Tungsten-rhenium alloys are utilized in industry for high-temperature applications such as incandescent-lamp wire and targets for high-power $x$-ray tubes. A slgniflcant amount of experience with the technology for their fabrication exists. Tungsten has been used for the following calculations, although a number of target configurations will be tested during the $R$ \& $D$ phase of the target-station development.

3.4.2 Energy Deposition. The energy deposition in tungsten was calculated using the computer code MAXIM ${ }^{4}$. The total energy deposited per proton vs target length is shown in Fig. 3-6.

The energy density deposited vs radius for Gaussian beams, is shown for $\sigma_{x}=\sigma_{y}=0.02 \mathrm{~cm}$ in F1g. 3-7, and $0.04 \mathrm{~cm}$ in Fig. 3-8. The radial distribution $1 s$ wider on the downstream face of the target due to the shower development. Also shown is the energy density expressed in Joules gm $^{-1}$ pulse for $2 \times 10^{12}$ protons per pulse, to be compared with the integral of the enthalpy reserve for tungsten. This quantity, the integral of the heat capacity from $20^{\circ} \mathrm{C}$ to a given temperature, is shown in F18. 3-9. A summary of this data is presented in Table 3-VI. 
Table 3-VI. ENERGY DEPOSITION IN TARGET

Target Material

Length

\section{$125 \mathrm{GeV}$ protons/pulse \\ Total Beam Energy \\ Repetition Rate \\ Beam Pulse Duration}

Energy Deposited/proton

Energy Deposited/pulse

Average Energy Deposited

Average Temperature

Beam Size ( $\sigma \mathrm{x}=\sigma \mathrm{y})$

Peak Energy Density/proton

Peak Energy Density/pulse

Peak Temperature rise

CERN Peak Energy Density

Peak Temperature rise

Average Temperature

SLAC Peak Energy Density

Peak Temperature rise

Average Temperature
Tungsten/Tungsten Alloys

$5 \mathrm{~cm}$

$3.0 \times 10^{12}$

60075 Joules

$0.5 \mathrm{~Hz}$

$1.6 \times 10^{-6} \mathrm{sec}$

$1.13 \mathrm{GeV}\left(1.81 \times 10^{-10}\right.$ Joules $)$

543 Joules

271.5 Watts

$<100^{\circ} \mathrm{C}$

$\begin{array}{cccc}0.02 & 0.03 & 0.04 \mathrm{~cm} & \\ 25.0 & 16.0 & 9.0 & \mathrm{GeV} . \mathrm{cm}^{-3} \\ 623.0 & 398.0 & 224.0 & \text { Joules. } \mathrm{gm}^{-1} \\ 3410.0 & 2670.0 & 1750.0 & { }^{\circ} \mathrm{C} \\ & >185 \text { Joules } \mathrm{gm}^{-1} \\ & 1500^{\circ} \mathrm{C} \\ & 800^{\circ} \mathrm{C} \\ & & \\ & 81 \mathrm{Joules} \mathrm{\textrm {gm } ^ { - 1 }} \\ & 600^{\circ} \mathrm{C}\left(\text { SLAC Report } 480^{\circ} \mathrm{C}\right) \\ & 600^{\circ} \mathrm{C}\end{array}$

The target used at the CERN Antiproton Source was designed for a peak energy density of 185 Joules $\mathrm{gm}^{-1}$ and a peak temperature rise of $1500^{\circ} \mathrm{C}$ above an ambient temperature of $800^{\circ} \mathrm{C}$. This target, which was made of rhenium, has been used for some time with no reported failures.

The SLAC Linear Collider has proposed to use a tungsten-rhenium target which will sustain peak energy densities of 81 Joules gm $^{-1}$ and maximum temperature rise of $600^{\circ} \mathrm{C}$ over an ambient temperature of $600^{\circ} \mathrm{C}$.

Based on the discussion above, the expectation is that after some target-development effort, one should be able to operate with an rms beam spot of $0.038 \mathrm{~cm}$.

3.4.3 Target Design. Elastic stress calculations for tungsten-rhenium alloys, which have the extreme temperature profiles which were generated by the energy-density deposition as discussed above show that a plastic zone (material compressed to above the yield point) will form concentric to the beam. The diameter of this zone is calculated to be $0.3 \mathrm{~mm}$ for a beam of $\sigma_{x}=\sigma_{y}=0.4 \mathrm{~mm}$. The size of this zone grows to approximately $0.6 \mathrm{~mm}$ for $\sigma_{x}^{x}=\sigma_{y}^{y}=0.2 \mathrm{~mm}$. 
The failure mechanism for a solid target is expected to proceed along the following sequence:

1. Development of a plastic zone with each heat pulse.

2. Flow of material within the plastic zone with each repeated pulse. The flow direction will be from the inside towards the faces of the target.

3. Swelling at the target face followed by surface cracking.

4. Surface cracks extending into the volume of the material.

In order to decrease the number of the thermal and stress cycles in the volume of material struck by the beam, the targets will be rotated continuously. This will result in a distribution of beam impact points around the circumference.

The target geometry of Fig. 3-10 is composed of a number of wedges, which decrease the cost of manufacturing the target compared with a circular rim. During the development stage, wedges will contain different designs for comparative testing. The target geometry of Fig. 3-11 is based on the powder technology used in the construction of high-power $x$-ray tubes. Because of the low average power deposited in the target, forced-air cooling should suffice for both geometries. Provision is being made to utilize, in the same target station, stationary targets of the CERN design.

\subsection{Target Hall}

The antiproton production target, proton beam dump and the lithium lens for antiproton collection will be located in a vault downstream of the final quadrupole focusing system in the $125-\mathrm{GeV}$ proton line. The dimensions of this vault are planned to be $7 \mathrm{ft}$ by $33 \mathrm{ft}$ with the floor located at $17 \mathrm{ft}$ below grade, as shown in Fig. 3-12a and 3-12b.

The upstream end of the hall is separated from the proton-beam transport tunnel by $3 \mathrm{ft}$ of steel shielding. Further shielding is placed around the external walls of the vault in the earth. Below the vault two separate impermeable membranes are used to collect irradiated ground water. Shielding configurations that limit the irradiations of the soil and the above-ground fluxes to permisgible values have been designed to allow operation at an intensity of $10^{13}$ protons per second.

Downstream of the upstream end of the shield, within the target vault, a $3 \mathrm{ft}$ high by $8 \mathrm{ft}$ long and $2 \mathrm{ft}$ wide volume of space is avallable for components. The space between this volume and the concrete walls and floors of the vault is filled with steel shielding. Access to the target station components is by raising one of a set of solid steel elevators into the Target Service Building. Each elevator segment extends $10 \mathrm{in}$. along 
the beam direction and $24 \mathrm{in.} \mathrm{transverse} \mathrm{to} \mathrm{the} \mathrm{beam.} \mathrm{Each} \mathrm{is} 5$ ft long in the vertical direction. The components are suspended from the bottom of the elevator. The $5 \mathrm{ft}$ length makes it possible to place electronics and control systems immediately above each elevator segment without danger of radiation damage. An additional $3 \mathrm{ft}$ of concrete is required to keep the above-ground radiation levels within the Target Service Building below the maximum permissible level. Within the Target Service Building and surrounding the vault, a shielded area incorporating thick windows will be used to exchange elevator segments remotely. Work will be performed on components with remote manipulators. The manipulators will be used to exchange targets routinely. Access to the electronics at the top of the elevator segments will be possible when beam is not being delivered to the target station.

The last component in the beam before the dump is a pulsed magnet to bend the $\bar{p}^{\prime} \mathrm{s}$ to the right by $3^{\circ}$, in order to separate them from the protons. The dump is a water-cooled graphite core $6 \mathrm{ft}$ long surrounded by a steel jacket which fills the inside of the vault. The steel extends for 22 feet along the deflected proton beam. The construction is similar to the dump developed for the Tevatron abort system.

\subsection{Antiproton Transport to the Debuncher}

A transport Iine has been designed to carry antiprotons to the Debuncher. It consists of modular FODO cells. The entire line will be capable of accommodating a $4 \%$ momentum spread and $20 \pi$ mm-mrad transverse acceptance in each dimension. At the end of the FODO channel, there will be $a 60^{\circ}$ bend. The line will then match into the Debuncher injection system as discussed in Sec. 4.5.1. 


\section{REFERENCES}

1. "Calculation of Yields for Antiproton Sources" A. Van Ginneken and C. Hojvat, in preparation.

2. E. Colton, "More on Antiproton Collectors," $\bar{p}$ note \#120, Fermilab (unpublished).

3. B. F. Bayanov et al., "A Lithium Lens for Axially Symmetric Focusing of High Energy Particle Beams," Nuc. Inst. Meth., 1909(1981).

4. "MAXIM - Program to Simulate Cascades in Bulk Matter." A. Van Ginneken, Fermilab FN-272 (January 1975).

5. "High Intensity Targeting Workshop," Fermilab April 28-30, 1980.

6. F. Krienen and F. Mills, Spreading the Hot Spot on the Target, $\bar{p}$ Note 70.

E. Colton, $\overline{\mathrm{p}}$ Note 107. 


\section{DEBUNCHER RING}

\section{1 Purpose of the Debuncher}

The primary purpose of the Debuncher is to reduce the large momentum spread of the 8-GeV $\bar{p}$ beam at production to $0.2 \%$ or less prior to injection into the Accumulator. This reduction is done by $r f$ bunch rotation and adiabatic debunching after the $\overline{\mathrm{p}}$ beam is injected into stationary 53-MHz buckets in the Debuncher. The debunching time is only slightly longer than $10 \mathrm{msec}$, and there are therefore nearly two seconds available for cooling before the beam is transferred to the Accumulator. Stochastic cooling of betatron amplitudes was found to be feasible, and a betatron cooling system to reduce the emittance by a factor 3 in both planes in $2 \mathrm{sec}$ is included in the design. Table 4-I gives parameters of the Debuncher.

\subsection{Rotation and Debunching}

In order to evaluate the effectiveness of the debunching process and thus to determine the momentum spread of 'p's that can be accepted at production, extensive computer simulations of the rotation and debunching sequences were carried out. The following features were included:

(i) The p's traversed a dispersionless drift of 100 to $400 \mathrm{~m}$ between the target and the first of cavity. The rf voltage was generated by a single cavity.

(11) Two values of $\eta$ were used. A value of -0.002 was used with an if voltage of $2 \mathrm{MV}$ and a value of -0.004 was used with an $\mathrm{rf}$ voltage of $4 \mathrm{MV}$. The results were the same for these two cases.

(iii) The bunches were allowed to rotate for 30 turns while the voltage was kept constant. The rotation was about $90^{\circ}$.

(iv) The voltage was then dropped to $81 \mathrm{kV}$ in 30 turns (or 50 isec) to form a bucket matched in shape to the beam bunch, which had evolved into a grossly distorted S-shape that extended over $\pm 90^{\circ}$ in synchrotron phase.

(v) The voltage was slowly reduced to $5 \mathrm{kV}$ or less.

A debunching time of 12 msec was chosen, since longer times did not significantly reduce the momentum spread while shorter times did increase the momentum spread. The final momentum spread is not sensitive to the other details of the time dependence of the $r f$ voltage. The $r f$ voltage program for the rotation and debunching operations is shown in Fig. 4- 1 for the case of $n=-0.004$ and $\Delta p / p=4 \%$. Figures 4-2 through 4-5 show the beam shape at the end of some of the steps for $\Delta p / p=3 \%$. If the debunching could 
be done without dilution, $95 \%$ of the beam would be contained within a momentum spread of $0.15 \%$. Computer simulations show that the debunched momentum spread is $0.3 \%$.

Calculations were done with smaller initial momentum spreads. The initial of voltage of $4 \mathrm{MV}$ and the final of voltage of $5 \mathrm{kV}$ were kept fixed for these calculations. These results, which are shown in Fig. 4-6, establish that the final momentum spread can be reduced significantly if the initial momentum spread is reduced by a relatively small amount. These calculations show that the debunched beam momentum spread is $0.2 \%$ if the bunched beam momentum spread is limited to 3\%. The momentum spread of the $p$ beam will be limited to $3 \%$ by collimation prior to injection into the Debuncher. The Debuncher 1 tself has a momentum aperture of over $4 \%$. It may be possible to reduce the debunched beam momentum spread further if the rf voltage can be reduced adiabatically to a value much less than $5 \mathrm{kV}$. The final momentum spread was not changed when a higher-harmonic $r f$ voltage was added. Harmonics of twice and three times the fundamental frequency were added in the simulations.

The factor of two dilution has several causes. It is caused in part by nonlinearities of phase oscillations of particles captured within $\mathrm{rf}$ buckets. The major causes, however, are the variations of $r^{2}$ of the particles and the momentum compaction factor $\gamma_{T}{ }^{2}$ with energy. These variations and the presence of sextupole corrections in the ring lattice were taken into account in the simulations.

After the $53 \mathrm{MHz}$ rf is turned off, a broad-band, low-frequency rf system is turned on to preserve the gap in the otherwlse debunched beam. A 200-nsec gap is needed if the beam is to be transferred to the smaller Accumulator without loss. The rf system to make this gap is described in Section 4.5 .2 .

\subsection{The Debuncher Ring Lattice}

The rf voltage needed for bunch rotation is proportional to $|\eta|$. If $|\eta|$ is less than 0.002 , the varlation of $|\eta|$ with momentum will degrade the final momentum spread. On the other hand, a larger value of $|\eta|$ helps the betatron cooling and is needed if momentum precooling is to be done in the future. As a compromise $\eta$ was chosen to be -0.004 . This choice makes $Y_{T}$ close to 12. Choosing $\gamma_{T}$ above the operating energy is desirable because it leads to strong focusing, which in turn leads to magnets with small apertures. The Debuncher Ring is made of 48 FODO cells, each of which is $11 \mathrm{~m}$ long. Long straight sections with zero dispersion have been included to accommodate pickups and kickers for stochastic cooling. These sections also provide the space for rf cavities and injection-extraction magnets.

A threefold symmetry for the lattice was chosen because it closely matches the Accumulator Ring, which also has threefold symmetry. This allows a reasonably convenient arrangement of straight sections with common equipment galleries. Figure 4-7 shows the overall Debuncher lattice. 
Figure 4-8 summarizes the parameters for a single cell. Figure 4-9 describes the dispersion-killer section and Fig. 4-10 describes a typical empty cell in the long stralght section. Each long straight section is made of six such cells. The packing factor, the ratio of bending length to circumference, is $24 \%$.

Each regular cell consists of a focusing quadrupole, a defocusing quadrupole, two dipoles and a correction package. The correction packages consist of two families of steering dipoles and sextupoles.

The steering dipoles have sufficlent strength to make local three-bump closed-orbit corrections of $\pm 5 \mathrm{~mm}$ displacement in the horizontal plane and $\pm 3 \mathrm{~mm}$ in the vertical plane. To do this correction, a steering dipole 30 $\mathrm{cm}$ long is placed next to each quadrupole. Their design specifications are given in Table 4-II.

The basic function of the sextupoles is to flatten the chromaticity in both planes so that the variation in tune across the aperture is less than 0.01 . Sextupoles which are $30 \mathrm{~cm}$ long are located next to each quadrupole in a region of significant dispersion. The sextupole specifications are given in Table 4-III. The variation of $Y_{T}$ with momentum is displayed in Fig. 4-11. Figure 4-12 shows the variations of the betatron tunes and Fig. 4-13 the variations of $\beta_{\text {pax }}$ and $\left(\alpha_{p}\right)_{\max }$. Figure $4-14$ gives the lattice functions across one-half period.

Vacuum pumps are located next to each quadrupole. The lifetime for an 8.9-GeV/c beam is calculated to be 2.4 hours when the average vacuum pressure is $10^{-6}$ Torr. This vacuum can be achieved without baking the chamber, so no provisions for baking are included in the design. The vacuum pipe will be carefully treated and cleaned during contruction and the vacuum is expected to be better than $10^{-6}$ Torr. Beam position monitors are located next to each quadrupole.

\subsection{Beam Transfer}

4.4.1 Injection. The beam enters the Debuncher in the horizontal plane at an angle of $120 \mathrm{mrad}$ to the closed orbit. A pulsed current septum located in a zero dispersion region deflects the beam onto an orbit parallel to the reference orbit and horizontally displaced by $50 \mathrm{~mm}$. A kicker in a zero-dispersion location $90^{\circ}$ in betatron phase away from the current septum kicks the beam onto the reference orbit. A schematic layout of these components is show in F1g. 4-15. The beam enters the upstream end of the septum magnet 30-cm from the reference orbit and exits the magnet $5 \mathrm{~cm}$ away from the reference orbit. The 30-cm displacement and the injection angle of 120 mrad make it possible to clear the quadrupole upstream of the current septum with ease. The infection channel has been designed to accommodate a beam with momentum spread of $4 \%$ and transverse emittances of $20 \pi \mathrm{min}$-mrad. 
The current-septum specifications are:

$\begin{array}{ll}\text { Length } & 4.0 \mathrm{~m} \\ \text { Bending Angle } & 120 \mathrm{mrad} \\ \text { Bending Radius } & 33.3 \mathrm{~m} \\ \text { Magnetic Field } & 8.9 \mathrm{kG} \\ \text { Aperture } & 5 \mathrm{~cm} \times 5 \mathrm{~cm}\end{array}$

The magnet is centered in the drift space between the two quadrupoles with approximately $30 \mathrm{~cm}$ clearance on each side.

The kicker specifications are:

$\begin{array}{ll}\text { Length } & 2.0 \mathrm{~m} \\ \text { Magnetic Field } & 500 \mathrm{G} \\ \text { Fall-off Time } & 150-200 \mathrm{nsec} \\ \text { Aperture } & 10 \mathrm{~cm}(\mathrm{~h}) \times 5 \mathrm{~cm}(\mathrm{v})\end{array}$

4.4.2 Extraction. The beam is extracted in the horizontal plane with a kicker and a current septum. The septum is in a zero-dispersion location, but the kicker is not. The magnets are separated by approximately $90^{\circ}$ of betatron phase advance. The system uses components that have the same specifications as the injection system.

\subsection{Radio Frequency Systems}

4.5.1 Antiproton Debuncher RF. The design criteria for the antiproton debuncher rf are:

(1) Voltage gain per turn $\geq 4 \mathrm{MV}$.

(ii) Maximum pulse length 100 usec (duty factor $5 \times 10^{-5}$ ).

(iii) RF voltage pulse fall time from $\geq 4 \mathrm{MV}$ to $80 \mathrm{kV}$ in 30 usec.

(iv) Pulse repetition rate $0.5 \mathrm{~Hz}$.

(v) Minimum voltage gain per turn $\leq 2 \mathrm{kV}$.

(vi) Adlabatic reduction of voltage from $90 \mathrm{kV}$ to $\leq 2 \mathrm{kV}$ in a time of 5 to 20 msec under program control.

A representative $r f$ voltage waveform is shown in Fig. 4-1. The design is simplified by the fact that the beam intensity will not exceed 10 lo particles when the $\mathrm{rf}$ voltage is on. Of the particles that emerge from the production target when it is struck by $3 \times 10^{12} 125-G e V$ protons, approximately $2 \times 10^{10}$ are within the acceptance of the beam transport and the Debuncher. Some $90 \%$ of these particles are mesons, of which $50 \%$ decay before reaching the injection straight section. After each successive turn, $63 \%$ of the remaining mesons decay. The remainder of the particles 
that reach the center of the infection straight section consist of muons, principally from pion decay, and electrons produced in showers in the target. The electrons lose $28 \mathrm{MeV}$ per turn through synchrotron radiation and spiral out of the vacuum chamber within 20 turns.

The large $r f$ voltage, low beam loading and small duty factor favor an accelerating cavity with a very high $Q$, hence a high shunt impedance, which requires very little of drive power. But the short fall time of the $r f$ voltage pulse is not obtained easily with a high-Q structure. Moreover, the physical size of high $Q$ structures operating in the 53-MHz region would require much larger enclosures than are under consideration.

A compromise structure, which contains an intermediate cylinder that reduces both the $Q$ and the physical size, is shown in Figure 4-16a. Figure 4-16b shows a SUPERFISH plot of the electric flelds within one-quarter of the structure. The entire volume of each cavity will be evacuated, with $\mathrm{rf}$ power coupled through two small ceramic windows at the top. The plate through which of power is introduced serves as a bedplate for the high-power driver amplifiers, which are mounted directly on top of the cavity and are completed enclosed. Thus high-power $r f$ transmission lines are not needed and $r f$ leakage is less likely. This may be important because $r f$ nolse could adversely affect stochastic cooling. Each cavity is driven by two relatively small $10-\mathrm{kN}$ triodes operating in a cathode-driven grounded-grid configuration. In this configuration, the tubes are capable of delivering very large peak of currents during the short pulse. Because the pulse is short and the duty factor is low, the average anode dissipation is only a few hundred watts.

The $r f$ voltage is reduced quickly by reversing the phase of the power-amplifier excitation and raising the excitation amplitude to a very high level. The high peak power capability of the tubes is used to "drive" the cavities off, even though the cavity time-constant is longer than the required turn-off time of $50 \mu \mathrm{sec}$.

The peak of voltage which each cavity can develop has been calculated to be larger than $600 \mathrm{kV}$. Elght of these 1.7-m long cavities easily provide the required $4 \mathrm{MV}$. The cavities can be installed in pairs in the 4-m spaces between quadrupoles in a long straight section.

In order to reduce the re voltage down to $2 \mathrm{kV}$ or less during the adiabatic-debunching part of the rf program, six of the cavities are turned off and two of the cavities are held at $50 \mathrm{kV}$ and thelr relative phases each changed by $90^{\circ}$ in opposite directions. The resultant $r f$ voltage gradually reaches a small value. This is done to avold instabilities at low $r f$ voltage that might arise from multipactoring.

In order to provide the structural rigidity necessary for complete evacuation and to reduce material costs the cavities will be constructed from aluminum. 
4.5.2 Gap-Preserving RF. Because the Debuncher circumference is larger than that of the Accumulator, antiprotons will be lost in the transfer to the Accumulator unless there is a gap in the Debuncher beam. Given the difference in circumference between the two rings and the need to allow for the fall time of the injection kicker, a gap greater than $200 \mathrm{nsec}$ is needed.

An adequate gap can be created in the beam by a "barrier bucket" that excludes particles from its interior. It is created by a voltage that traces out a single complete sinusoidal oscillation for one-quarter of the rf period and then becomes zero for the remaining three-quarters of the period. A sketch of this waveform is shown in Fig. 4-17. The voltage waveform repeats itself every turn. The phase of the voltage is chosen so that it establishes an unstable fixed point between two back-to-back half-bucket separatrices. If the bucket height exceeds the beam energy spread, the beam will be forced away from the unstable fixed point by a distance determined by the ratio of the bucket height to the energy spread. The $r f$ voltage is chosen so that the beam is excluded from a region between $\pm \pi / 2$ radians in the "barrier bucket". This will create a gap of $221 \mathrm{nsec}$, as shown in Fig. 4-17. The peak amplitude required is $450 \mathrm{~V}$. The Fourier series expansion of such a voltage waveform is given by

$\infty$

$$
V(t)=450 \frac{2 h}{\pi}{ }_{n=1} \frac{\sin \pi n / h}{h^{2}-n^{2}} \sin \left(n \omega_{0} t\right)
$$

where $\omega_{0}=2 \pi f=3.55 \times 10^{6} \mathrm{rad} / \mathrm{sec}$ and $h=4$. At frequencies above the 31 st harmonid (19.5 MHz) the required amplitudes are reduced by more than three orders of magnitude. Amplifiers with a bandwidth between 0.1 and $30 \mathrm{MHz}$ which are capable of delivering $300 \mathrm{~W}$ in a 50-ohm load are readily available. The required rf system can be broken into 150-v units, each consisting of a ceramic gap loaded with a 50-ohm resistor and surrounded by a shielding enclosure containing about ten large MnZn ferrite rings. The average power delivered to this system is very small. Since the physical size of each unit is dictated by the volume of ferrite required, each unit is $0.5 \mathrm{~m}$ in length.

An additional bonus associated with installation of the "barrier bucket" $r f$ system is that it can be used in the "normal" $h=4$ mode to accelerate or decelerate $8 \mathrm{GeV}$ protons across the ring aperture in tune-up testing of closed orbits and stochastic-cooling systems.

\subsection{Magnet and Power Supply Systems}

The Debuncher magnet ring consists of 24 regular cells, 6 dispersionkiling cells and 18 special cells in the straight sections. By bullding dipoles and quadrupoles with different lengths, it is possible to excite the quadrupoles to the same gradient of $9.78 \mathrm{~T} / \mathrm{m}$ and the dipoles to the same fleld of $1.528 \mathrm{~T}$ with the same currents. There are two 
different length dipoles that have the same profile. There are nine different length quadrupoles, all but one of which have the same profile. The most common quadrupole has the same profile as the small Accumulator quadrupole, while the exception, QD2, has the same profile as the large Accumulator quadrupole. There are six quadrupoles with this profile.

A beam with a 4\% momentum spread and an emittance of $20 \pi$ mm-mrad requires a good-field aperture of $75 \mathrm{~mm}(\mathrm{~h})$ by $50 \mathrm{~mm}(\mathrm{v})$ in the dipoles and $80 \mathrm{~mm}$ by $50 \mathrm{~mm}$ in all but quad QD2, which requires a vertical aperture of $63 \mathrm{~mm}$. The good-field region of the dipole has been designed to be $100 \mathrm{~m} \times 60 \mathrm{~mm}$. The sagitta of the beam is $32 \mathrm{~mm}$ and the long dipoles are therefore curved to match the orbit. The properties of these magnets are tabulated in Table 4-IV.

The magnet ring is powered by three separate power supply buses, one for the dipole and one each for the regular focusing and defocusing quadrupoles. Quadrupoles in the long straight sections are powered separately. Each correction element has its own power supply. The estimated power consumption of the ring is $2 \mathrm{MW}$.

\subsection{Betatron Cooling}

4.7.1 Cooling System Parameters. The Accumulator lattice design discussed in section 5 of this report must satisfy the stringent requirements on the beam size that are imposed by the stack-tail stochastic cooling system. The cooling rate is degraded for particles that have large betatron amplitudes and the sensitivity of the cooling system improves if the gap height of the pickups and kickers is reduced. Betatron cooling is therefore included in the Debuncher Ring. The $20 \pi \mathrm{mm}$-mrad beam will be cooled to match the $10 \pi \mathrm{mm}-\mathrm{mrad}$ acceptance of the Accumulator lattice.

The betatron cooling systems in the Debuncher are designed to reduce each of the two transverse emittances by a factor of 3 in $2 \mathrm{sec}$. The parameters that control their design can be illustrated by the following short analysis. The equation which governs the change of beam emittance with time is

$$
\frac{d \varepsilon}{d t}=\frac{W}{N}\left[\left(-2 g+g^{2} M\right) \varepsilon+g^{2} B \varepsilon_{0}\right] \text {, }
$$

The numerical example will be carried out for a bandwidth $\mathrm{W}$ of $2 \mathrm{GHz}$ and $\mathrm{N}$ $=10^{8}$ particles. The mixing factor $M$ and the noise-to-signal ratio $B$ in Eq. (4-1) are

$$
M=\frac{f_{0} \Lambda}{2 W n \Delta p / p}
$$




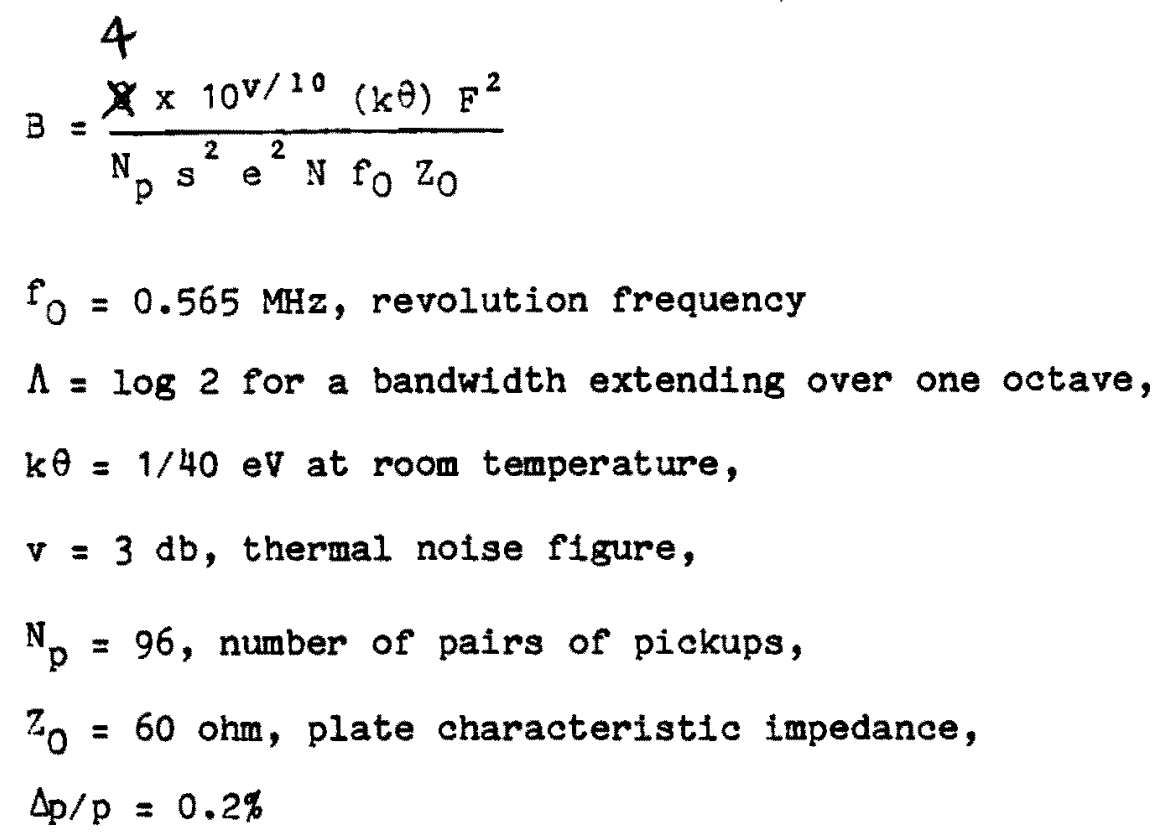

The sensitivity factor $s$ that depends on the geometry of the plates, was chosen to be 0.8 . F, the ratio of the gap between plates to the diameter of the beam at the pickup, was chosen to be 1.15. For these parameters the mixing factor $M$ is 12 , and the noise to signal ratio $B$ is 9.

Equation (4-1) has the solution

$\varepsilon=\left(\varepsilon_{0}-\varepsilon_{\infty}\right) e^{-t / \tau}+\varepsilon_{\infty}$

where $\varepsilon_{0}$ is the inttial beam emittance and $\varepsilon_{\infty}$ the equilibrium value due to thermal noise. A reduction of a factor 3 in betatron emittance from $20 \pi$ to $7 \pi \mathrm{m}$-mrad can be obtained if $\mathrm{g}$ is chosen to be 0.015 . is

The relation between the electronic gain $g_{A}$ and the dynamical gain $g$

$$
g=\frac{c s^{2} N e^{2} f_{0} z_{0} \sqrt{N_{k} N_{p}}}{3 \pi W \varepsilon_{0} F^{2}} g_{A}
$$

Where $N_{k}=N_{p}$ is the number of kickers, $c$ is the velocity of light, $E$ is the total particle energy, and $\beta$ the relativistic velocity factor. For this example the amplifier gain $\mathrm{g}_{\mathrm{A}}$ is $6 \times 10^{6}(136 \mathrm{db})$, and the thermal power $\mathrm{P}_{\mathrm{N}}$ is $10^{\mathrm{V} / 10}(\mathrm{k} \theta) \mathrm{Wg}_{\mathrm{A}}^{2} \leq 500 \mathrm{~W}$. Schottky power is negligible in
comparison.

The highest frequency which appears to be compatible with the beam and vacuum chamber sizes is $4 \mathrm{GHz}$. For this reason, a bandwidth of $2-4 \mathrm{GHz}$ was chosen for the betatron cooling system. A smaller bandwidth would lead to a slower cooling rate. The horizontal and vertical systems each have 96 pickups and 96 klckers. The horizontal and vertical pickups are separated 
longitudinally as are the kickers of each system. Pickups will be loop couplers of the type under development at LBL.

4.7.2 Pickup and Kicker Design. The preliminary R\&D work at LBL suggests that the pickup spacing will be $4 \mathrm{~cm}$ or slightly greater. It is planned to place 16 pickups in a 75-cm long subassembly. Two of these subassemblies will be placed in a 1.5-m module. Because thermal noise is such an important effect, consideration is being given to cooling the pickups and combiners to $80 \mathrm{~K}$ or lower. In addition, consideration is being given to cooling the preamplifiers. The preceding numerical example does not include these potential reductions in $B$.

Although the criteria on the vertical and horizontal beam size within each plckup or klcker module have been established, a specific choice of lattice functions within the cooling straight sections is still under consideration.

4.7.3 Effect of the gap on betatron cooling. The effect of the "barrier bucket" on stochastic cooling has been considered. Recall that the "barrier bucket" establishes a gap in an otherwise-continuous debunched beam. The process of creating this bucket adiabatically after betatron cooling, is time consuming, it is planned to turn the barrier bucket on as soon as the debunching is completed. This raises the question of the effect that such a gap has on the betatron cooling. Excitation of $a$ beam pickup by the gap in the beam is equivalent to excitation by a single bunch in the ring with a current distribution equivalent to the missing current in the gap, as shown in Fig. 4-17. If the current is described by a $\cos ^{2}$ dependence where it is changing and if the gap length is related to the total bucket length by a factor $k$, then the Fourier series representing the current distribution is

$$
i(t)=i_{d c}\left\{1+\frac{2 h}{(k+1) \pi} \sum_{n=1}^{\infty} \frac{(\sin n k \pi / h+\sin n \pi / h)}{n\left(1-(1-k)^{2}(n / h)^{2}\right)^{2}} \cos n \omega_{0} t\right\} .
$$

For $h=4, k=0.5$, and $\omega_{0}=3.5 \times 10^{6}$, the maximum amplitude of signals in the region of $1 \mathrm{GHz}(\mathrm{n} 91800)$ is about $2 \times 10^{-8}{ }_{1} \mathrm{dc}$, which is much smaller than the thermal noise.

\subsection{Equipment Arrangement}

The layout of equipment is shown in Fig. 4-7. The major 53- $\mathrm{MHz} \mathrm{rf}$ system is made of 4 palrs of cavities. Each pair has a length including flanges which special vacuum pumps, which can be placed in a 4.7-meter long straight section. 
Table 4-I. THE DEBUNCHER RING

Kinetic Energy

$\eta=r_{t}^{2}-r^{2}$

$\gamma_{t}$

Average Radius

RF Frequency

Maximum RF Voltage

Number of p-bunches infected

Harmonic Number

Beam Gap for Injection Kicker

Momentum Aperture, $\Delta p / p$

Betatron Acceptance, $\mathrm{H}$ and $\mathrm{V}$
8.0 GeV

$-0.00374$

11.72

$81.3 \mathrm{~m}$

$53.1035 \mathrm{MHz}$

$4 \mathrm{MV}$

80

91

220 nsec

$4 \%$

$20 \pi$ mm-mrad 
Table 4-II. STEERING DIPOLES

$\begin{array}{lll} & \text { Horizontal } & \text { Vertical } \\ \text { Maximum closed orbit dist. } & \pm 5 & \pm 3 \mathrm{~mm} \\ \text { Deflection } & 0.31 & 0.19 \mathrm{mrad} \\ \text { Effective Length } & 0.30 & 0.30 \mathrm{~m} \\ \text { Maximum Field } & 614 & 368 \mathrm{G} \\ \text { Gap } & 40 & 80 \mathrm{~mm} \\ \text { Ampere-turns } & 1956 & 2344 \mathrm{~A}-\mathrm{turns} \\ \text { Current Density } & 1.0 & 1.0 \mathrm{~A} / \mathrm{mm}^{2} \\ \text { Current } & 10 & 10 \mathrm{~A} \\ \text { Turns } & 196 & 235 \mathrm{turns} / \mathrm{mag} \\ \text { Resistivity } & 0.28 & 0.33 \mathrm{Ohm} / \mathrm{mag} \\ \text { Voltage } & 2.8 & 3.3 \mathrm{~V} / \mathrm{mag} \\ \text { Thermal Loss } & 28 & 33 \mathrm{~N} / \mathrm{mag} \\ \text { Total Number } & 48 & 48 \mathrm{~m}\end{array}$

Table 4-III. SEXTUPOLE MAGNETS

$\underline{S F}$

Number

Strength for zero chromaticity $B^{\prime \prime} / B \rho$

Bore Radius

Core Length

Estimated Effective Length

Maximum Field, $B^{n}$

Ampere-turns

Current

Turns

Conductor Size

Conductor Area

Current Density

Resistance

Voltage Drop

Thermal Loss

Power Supply

Current

Voltage

Power
24

0.92

50.0

0.265

0.30

96

1600

44.5

36

$5.8 \times 5.8$

24.7

1.8

0.122

5.4

0.24

44.5

130

5.8
$\underline{S D}$

30

$1.5 \mathrm{~m}^{-2}$

$50.0 \mathrm{~mm}$

$0.265 \mathrm{~m}$

$0.30 \mathrm{~m}$

$238 \mathrm{~T} / \mathrm{m}^{2}$

$4000 \mathrm{AT} / \mathrm{pole}$

$111 \mathrm{~A}$

36 Turns/pole

$5.8 \times 5.8 \mathrm{~mm}^{2}$

$24.7 \mathrm{~mm}^{2}$

$4.5 \mathrm{~A} / \mathrm{mm}^{2}$

$0.122 \mathrm{ohm} / \mathrm{mag}$

$13.6 \mathrm{~V} / \mathrm{mag}$

$1.51 \mathrm{~kW} / \mathrm{mag}$

$111 \mathrm{~A}$

$\checkmark$

$45.5 \mathrm{~kW}$ 
Table 4-IV. DEBUNCHER MAGNETS

$\begin{array}{cccccc}\text { Dipoles } & \text { Number } & \text { Arc length } & \text { Strength } & \text { Gap } & \text { Goodfield width } \\ \mathrm{B}_{1} & 48 & 2.258 \mathrm{~m} & 1.528 \mathrm{~T} & 60 \mathrm{~mm} & 100 \mathrm{~mm} \\ \mathrm{~B}_{2} & 12 & 1.129 \mathrm{~m} & 1.528 \mathrm{~T} & & \end{array}$

\section{Quadrupoles}

\begin{tabular}{|c|c|c|}
\hline$Q F$ & 27 & $1.03 \mathrm{~m}$ \\
\hline$Q D$ & 30 & 1.00 \\
\hline$Q F_{1}$ & 3 & .22 \\
\hline $\mathrm{QD}_{1}$ & 6 & .13 \\
\hline$Q F_{2}$ & 6 & .35 \\
\hline $\mathrm{QD}_{2}$ & 5 & .78 \\
\hline $\mathrm{QF}_{3}$ & 6 & .78 \\
\hline$Q D_{3}$ & 6 & 1.29 \\
\hline $\mathrm{QD}_{4}$ & 6 & 1.09 \\
\hline
\end{tabular}

Gradient
$9.7845 \mathrm{~T} / \mathrm{m}$
$"$
$"$
$"$
$"$
$"$
$"$

Poletip Radius $43.27 \mathrm{~mm}$

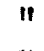

II

II

it

$84.1 \mathrm{~mm}$

43.27

" 


\section{ACCUMULATOR RING}

\subsection{Stochastic Cooling in the Accumulator}

5.1.1 Introduction and Summary of the Stochastic Stacking Process. The first function of the Accumulator is to accept a pulse of $\bar{p}$ 's every 2 seconds. In order to make room for this pulse, the $\bar{p}$ 's must be compressed (cooled) into a smaller area of phase space. A technique to accomplish the simultaneous $\overline{\mathrm{p}}$ accumulation and compression has been developed at CERN and is the basis of this design. The technique consists of establishing a stack of $\bar{p}$ 's with an energy density that rises approximately exponentially from the injection density (the low-density end is referred to as the "stack tail") and then culminates in a roughly Gaussian high-density region (the "core"). The density profile of the proposed system is shown in Fig. 5-1. Betatron amplitudes are also cooled during the stacking process. The design criteria for the accumulation process are given in Table 5-I.

Table 5-I. ANTIPROTON STACK PARAMETERS

Injected Pulse

Number of $\bar{p} ' s$

$7 \times 10^{7}$

$\Delta \mathrm{p} / \mathrm{p}$

$0.2 \%$

Horizontal and vertical emittance

$10 \pi \mathrm{mm}-\mathrm{mrad}$

Time between injections

$2 \mathrm{sec}$

Fraction of beam accepted

$>85 \%$ of infected pulse

FIux

$3 \times 10^{7} \overline{\mathrm{p}} / \mathrm{sec}$

Final Stack

Number of $\bar{p}$ 's

$4.3 \times 10^{12}$

$\Delta \mathrm{p} / \mathrm{p}$

Horizontal and vertical emittance

$0.1 \%$

$2 \pi$ mm-mrad

Peak density

Core width (Gaussian part)

Total stacking time

$1 \times 10^{5} \mathrm{eV}^{-1}$

1.7 MeV (rms)

4 hours

The stochastic stacking system consists of pickup electrodes, an amplifier system with electronic filters and phase-compensation networks and kicker electrodes. Each particle produces an electronic signal that, when applied to the kicker, changes its momentum in the direction of the momentum of the core. The signal of each given particle thus produces a kick that tends to cool the beam into a small momentum width around the core. Other particles in the beam with approximately the same revolution frequency produce random kicks on the given particles and cause diffusion or heating. The interesting systems, of course, are those in which cooling dominates over heating. 
Momentum cooling is usually described in terms of the Fokker-Planck equation

$$
\frac{\partial \psi}{\partial t}=\frac{\partial}{\partial E}\left[-F \psi+\left(D_{0}+D_{1}+D_{2} \psi\right) \frac{\partial \psi}{\partial E}\right]
$$

where $\psi=\partial N / \partial E$ is the particle density, $F$ is the coefficient of the cooling term, $D_{0}$ is the coefficient of the heating term due to intrabeam scattering (described later), $D_{1}$ is the coefficient of the heating term due to thermal noise, and $\mathrm{D}_{2}$ is the coefficient of the heating due to other particles. A derivation and discussion of this equation are given by Mohl et al. 1

A simplified version of the Fokker-Planck equation has been used by van der Meer ${ }^{2}$ to describe the stacking process. It is assumed that the voltage on the kicker is exactly in phase with the particles that created it, that there is no amplifier thermal noise or intrabeam scattering, the feedback gain is independent of harmonic number, and that there are no beam-feedback effects. While none of these assumptions is justified in the proposed system, the simplified discussion yields semi-quantitative results that can form the basis of a design. ${ }^{3}$ Following van der Meer, the flux can be written as

$$
\begin{gathered}
\Phi=-\frac{V}{T} \Psi-\mathrm{AV}^{2} \Psi \frac{\partial \psi}{\partial \mathrm{E}} \\
\text { cooling heating } \\
\text { term }
\end{gathered}
$$

where $N(E, t)$ is the number of particles with energy less than $E$ and

$$
\begin{aligned}
& \Phi=\frac{\partial N}{\partial t} \\
& \psi=\frac{\partial N}{\partial E},
\end{aligned}
$$

from which the Fokker-Planck equation 
$\frac{\partial \psi}{\partial t}=\frac{\partial \Phi}{\partial E}$

follows. Here $V=V(E)$ is the average energy loss per turn and $T$ is the revolution period. The constant A describes the strength of the heating term and is given by

$$
A=\frac{\beta P \Lambda}{4 T^{3} W^{2}|\eta|},
$$

where $\beta=v / c, p$ is the momentum, $T$ is the revolution period, $W$ is the bandwidth $=f_{\max }-f_{\min }, \Lambda=\ln \left(f_{\max } / f_{\min }\right)$, and $\eta=\gamma_{t}^{-2}-\gamma^{-2}$. If the

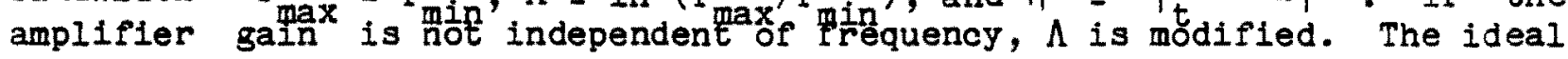
gain profile, in fact, rises linearly with frequency, but the exact value of $\Lambda$ is not important for this discussion.

The Fokker-Planck equation is nonlinear in $\psi$ and it is therefore usually solved numerically. Stationary solutions, $\Phi(E, t)=\Phi_{0}=$ constant, can often be found by elementary methods. These solutions are useful because in the stack tail between the core region and the injection region, the actual time-dependent solution is normally very close to the steady-state solution.

Consider $\Phi_{0}=$ constant and $\psi(E, t)=\psi(E)$, and ask what the shape of the voltage profile $V(E)$ should be. The answer, as given by van der Meer, is to maximize $d \psi / d E$ everywhere and thus minimize the energy aperture which is required. This choice also minimizes the total schottky power in the amplifier. The solution is

$$
V(E)=-\frac{2 \Phi_{0} T}{\Psi(E)}=-\frac{2 \Phi_{0} T}{\Psi_{1}} e^{-\left(E_{1}-E\right) / E_{d}}
$$

where $\Psi_{1}$ is the initial stack-tail density at $E_{1}$ and $E_{d}$ is the characteristic energy

$$
E_{d}=-4 A \Phi_{0} T^{2}=-\frac{B P \Lambda \Phi_{0}}{T W^{2}|n|} .
$$

This equation exhibits the major design consideration. We know that d $\psi / d E$ is maximized by a gain profile $V(E)$ that is exponential in energy. The 
exponential slope $d \psi / d E$ is maximized by minimizing $E_{d}$. In the stack tail, we want a density increase of 1000 , so we require a minimum momentum aperture of $\Delta \mathrm{p} / \mathrm{p}=\ln (1000) \mathrm{E}_{\mathrm{d}} / \mathrm{p}=6.91 \mathrm{E}_{\mathrm{d}} / \mathrm{p}$. If we want to limit the aperture $(\Delta p / p)$ required for the stack tail to $3 / 4 \%$, then $E_{d}<0.001 p \simeq 10$ $\mathrm{MeV}$ for $p=3.9 \mathrm{GeV} / \mathrm{c}$. Since we want to have $\Phi_{0}$ as large as possible, we must make $\mathrm{E}_{\mathrm{d}}$ small by choosing $T W^{2}|\eta|$ to be as large as possible.

We have chosen to work with a maximum frequency of $4 \mathrm{GHz}$ for the purposes of this report, but we intend to use frequencies up to $8 \mathrm{GHz}$ for core cooling if our research program indicates that $8 \mathrm{GHz}$ cooling systems are practical. The stack-tall system was chosen to have a maximum frequency of $2 \mathrm{GHz}$. As described below, the cholce of frequency dictates the cholce of $\eta$. By limiting the stack-tall system to $2 \mathrm{GHz}$, we are able to use a relatively high $\eta(\eta=0.02)$. The high value of $\eta$ is useful for the core-cooling systems, where the core cooling times are inversely proportional to $n$.

The maximum value of $\eta$ is limited once we choose $f_{\text {max }}$. For a number of reasons, it is required that $n(\Delta p / p) f_{\max } T<\delta$, where ${ }^{\prime} \delta$ is some number of order unity. The things that determine the value of $\delta$ are:

(i) The Schottky bands must not overlap in a system that uses electronic filters for gain shaping. In such a system, the particle energy 13 sensed in the electronics by the connection between energy and the harmonics of the revolution frequency. If the relationship is not unique, it is difficult and probably impossible to design appropriate filters. In this case, $\delta=1$ and $\Delta \mathrm{p} / \mathrm{p}=18$ so $\eta<0.03$ with $\mathrm{T}=1.6 \mu \mathrm{sec}$.

(i1) The phase shist between $P U$ and kicker must not vary across the momentum band more than about $\pm 45^{\circ}$. If $P U$ and kicker are exactly opposite in the Accumulator Ring, then $\delta=0.5$. If the stack-tail system is divided into subsystems with $\Delta p / p=1 / 3 \%$, then this constraint applies to each system individualiy, so $n$ $<0.05$.

(111) For reasons described below, the filters must have the peak of their response at the tail end and a notch in the core. Between Schottky bands, of course, the response must rise from the notch in the core back to the peak value. Since the rise back to the peak value cannot be done infinitely sharply, at least without undesirable phase characteristics, this requirement is more severe than 1) above. We have found that $n$ $=0.02$ is a suitable value for a maximum frequency of $2 \mathrm{GHz}$.

Since an increase or decrease in $T$ requires a corresponding change in $\eta$, the cooling does not depend on the size of the Accumulator Ring. We have chosen a Booster-sized ring $(T=1.6 \mu \mathrm{sec})$ because it is large enough to accommodate the cooling-system hardware and can run at $8 \mathrm{GeV}$, a good energy for injection of p's into the Main Ring. 
5.1.2 Summary of Design Considerations. We have chosen $E_{d}=0.001 p=10$ MeV to keep the required momentum aperture sufficiently small. The product If ${ }_{\text {max }} \eta$ is fixed by the requirement of a minimum spacing between schottky banas. We have chosen $W=1 \mathrm{GHz}$ with $\mathrm{f}_{\mathrm{max}}=2 \mathrm{GHz}$ to yield a somewhat higher value of $\eta(0.02)$ than would be the max âse if we chose a higher frequency.

5.1 .3 Bullding the Exponential Gain Profile. Once the parameters of the stack tail have been chosen, the next step is to build the required gain profile. We use a combination of two techniques. The first technique is to sense the particle momentum by sensing its position in a region of high momentum dispersion. The position sensitivity of the strip-line pickups we plan to use at midband $1 \mathrm{~s}^{4}$

$$
s(x)=\frac{1}{\pi} \operatorname{Atan} \frac{\sinh \pi w / 2 g}{\cosh \pi x / g},
$$

where $w$ is the width of the plates and $g$ is the gap between them. For large $x$ this becomes $s(x) \approx e^{-\pi x / 8}$. In our case, the momentum dispersion is $\alpha_{p}=9 \mathrm{~m}$ and we have chosen $\mathrm{g}=3 \mathrm{~cm}$. Thus for large $\Delta \mathrm{E}$,

$$
V(E)=e^{-|\Delta E| / E^{*}} Q(E),
$$

where $\Delta E$ is the difference between energy $E$ and the energy where the pickup response is centered, $E^{*}=10 \mathrm{MeV}$, and $Q(E)$ is the filter response. In our system we use the pickups in the region where the fallof is not truly exponential, but the system can be characterized roughly by an $\mathrm{E}^{*}$ for the pickup response of approximately $15 \mathrm{MeV}$.

The main purpose of the filters is to reduce the thermal noise in the core. In order to maintain a flux of $3 \times 10^{7}$ into the stack tail, an amplifier system with very high gain (150dB) is required. Even with preamplifiers with low noise flgures (2dB), the thermal noise produces an rms voltage of approximately $3000 \mathrm{~V} /$ turn. This noise voltage is (perhaps surprisingly) tolerable in the tail where the average (cooling) voltage gain is about $10 \mathrm{v} /$ turn. In the core region, where the cooling voltage is a few $m V / t u r n$, this noise voltage must be reduced to a tolerable level. The filter does this by making a notch at all harmonics of the revolution frequency of the particles in the core. The filter also does some gain shaping in the tail region. The filters used are composed of a series of notch filters similar in concept to those used at CERN. A schematic diagram of the individual component filters is given in Fig. 5-2. The response of these filters is given by 
$\frac{v_{\text {out }}}{v_{\text {in }}}=\frac{Z_{L} / R}{\operatorname{coth}(\alpha \ell+j \beta \ell)+z_{L} / R}$,

where $\mathrm{V} / \mathrm{V}_{\text {in }}$ is the voltage gain, $\mathrm{Z}$ is the impedance of the shorted

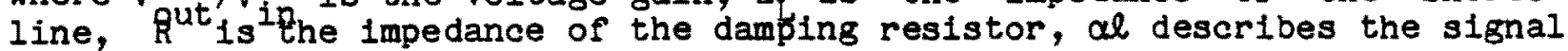
loss, $\alpha l=(\alpha l)$ $V(f / f, f)$, and $B$ describes the resonant frequency, $B l=$ $2 \pi \ell / \lambda=2 \pi \ell f / v, v$ is $v$ the phase velocity of the line, and $f$ is the frequency.

The shape of the gain curve at the infection end is purposely different than the ideal exponential curve, becoming flat in the injection region because of several practical considerations:

(i) It is desirable to keep the electronic gain between schottky bands as low as possible. The gain between Schottky bands does not affect the cooling process, but the thermal power between Schottky bands is signtflcant.

(i1) To maximize the ratio of Schottky signal to thermal noise, it is desirable to place the pickups so they have maximum sensitivity to the freshly injected beam, i.e., so they operate in the non-exponential region.

(1ii) The exponential gain profile minimizes Schottky power only in the approximation that beam is injected in a steady state manner. In fact, each newly injected pulse substantially alters this picture since the density of particles will differ by factors of 2 or 3 from the steady state situation. By making the gain profile flatter in the injection region, we can reduce the required Schottky power immediately after a new pulse is injected.

The stack-tail system we have designed consists of two sections of pickups and associated amplifiers and filters. The two sections make it possible to control undesirable phase shifts and thermal noise in the tail. A block diagram of the system is shown in Fig. 5-3. The number of pickups was chosen to be a large as possible to minimize the thermal-noise to Schottky-signal ratio and to minimize the total thermal power. In order to keep the betatron oscillations from substantially affecting the momentum cooling, the betatron amplitudes must be limited. To achieve the desired gain profile, the pickups have a plate separation of $3 \mathrm{~cm}$. Calculations indicate that the beam size should be less than $2.4 \mathrm{~mm}$ to avold trouble with betatron motion. For an emittance of $10 \pi$ mm-mrad, the 3 function at the pickup must be $15 \mathrm{~m}$ or less. This requirement limits the pickup straight section to $15 \mathrm{~m}$ in length and a total of 200 plckups. The function of the subtracting pickups is discussed in the next section. The number of kickers was chosen to fill the straight section across from the pickups to minimize total power (inversely proportional to the number of kickers). The gain profile achieved with this sytem is shown in Fig. 5-4 abc for the Schottky bands at $1.1,1.5$ and $1.9 \mathrm{GHz}$. 
5.1.4 Signal Suppression and Stability. An important aspect of the cooling process, when using high-gain cooling systems, is signal feedback via the beam. A signal of frequency $\omega$ will modulate the beam at frequency $\omega$ and this modulation will be sensed at the pickup. Thus the cooling system forms a closed-loop feedback system. This feedback system is analogous to amplifier systems with conventional electronic feedback. An expression for the beam feedback has been given by van der Meer ${ }^{5}$ and independently by Ruggiero. ${ }^{6}$ An approximate expression is

$$
F=\frac{I_{p}}{V}=\left\{j e f_{0}^{2}, \tilde{P} \int \frac{C P}{n k\left(E-E^{\prime}\right)} \frac{d \psi}{d E^{\prime}} d E^{\prime} \quad-\frac{\pi e f_{0}^{2}}{n|k|} C P \quad \frac{d \psi}{d E}\right\},
$$

where $P$ denotes the princlpal value of the integral, $I_{F}=$ induced current at the frequency $f=n f_{0}$ in the plckup due to modulation caused by voltage $V$ on the kicker, $f_{0}$ is the revolution frequency corresponding to energy $E$, $n$ is the harmonic number, e is the unit charge, $j=V_{-1, k}=2 \pi \mathrm{df} / \mathrm{dE}, \mathrm{C}$ is the phase factor due to transit time differences between pickup and kicker, and $P=P(f, E)$ is the pickup sensitivity. $P$ depends on $E$ ' because $P$ depends on particle position, which depends on $E^{\prime}$ (usually exponentially). The dependence of $P$ on $f$ comes from electrical properties and is usually weak.

This approximation is valid when the Schottky bands are well separated and is a poor approximation for quantitative results for the system described here. Nonetheless, it is sufficient to expose the main features of the physical process.

The closed-loop gain of the system is given by $G^{\prime}=G /(1-F G)$, where $G$ is the open-loop gain $\left(G=V / I_{\text {f }}\right)$ of the electronics going from pickup to klcker. If the real part of FG if less than zero, then $G^{\prime}<G$ and the cooling signal is suppressed. If the real part is greater than zero the signal may be enhanced. If the real part of FG $>1$, when the imaginary part is zero, the system is unstable. (This situation is completely analogous to the case of conventional electronic circuits with feedback).

In the approximate expression for $F$, one sees that there is a resistive (energy-absorbing) component of the beam response proportional to the gradient of the density at the driving frequency and a reactive component that depends on the asymmetry of the gradient about the driving frequency. It would be wrong to conclude, however, that the resistive term is the more important when looking at system stability. Both terms must be considered because the open-loop gain function $G$ is a complex quantity; it unavoldably contains phase shifts from the filters and differences in time delays between pickup and kicker.

In fact, in the stack tail, the feedback can be dominated by the contribution from the particles in the core where $\mathrm{d} \psi / \mathrm{dE}$ is very large - $10^{4}$ 
times larger than in the tail. Fortunately, $d \psi / d E$, which is increasing exponentially, is multiplied by the pickup response, which is decreasing exponentialiy. The rate of exponential increase of $\mathrm{d} \psi / \mathrm{dE}$ depends on the total gain profile, $1 . e$. , the product of pickup and filter response. The damping of $d \psi / d E$ in the feedback integral, however, depends only on the plckup response. Thus, it is important that the filter gain profile not be too sharp compared with the pickup in order to avold severe problems with stability and signal suppression. The importance of the relative amounts of the gain profile derifved from filters and pickups has been pointed out previously by Sacherer.

An additional suppression of signal from particles in the core is provided by the subtracting pickups in each section. These pickups are placed closer to the core and normalized so that their sensitivity to the core region is equal and opposite to the sensitivity of the main pickups. In the stack tail, however, they subtract less than $10 \%$ of the signal. Several subtracting pickups followed by substantial attenuation are required to avoid having the subtracting pickups appreciably affect the amount of thermal noise in the plckups. Immediately after injection, the signal suppression is substantially larger because of the large values of $\mathrm{d} \psi / \mathrm{dE}$ created by the RF stacking process. The gradients quickly (after 200 msec) smooth out because of the diffusion terms in the Fokker-Planck equation. It appears that during the first $200 \mathrm{msec}$ of the injection cycle, it may be necessary to reduce the amplifier gain in order to maintain beam stability. This gain reduction has been taken into account in computer simulations and, in any event, is only of minor importance.

5.1.5 Core Cooling. The same Fokker-Planck equation that describes the stack-tail system also describes the core system. In fact, the distinction between core and tall cooling systems is somewhat arbitrary. The asymptotic distribution is given by

$$
\Phi=\underline{E \Psi}+\left(D_{0}+D_{1}+D_{2} \Psi\right) \frac{\partial \Psi}{\partial E}=0
$$

The cooling coefficient $F$ has a zero at the peak of the core and a slope proportional to $g\left(E-E_{c}\right)$ where $g$ is the electronic gain and $E_{c}$ is the energy at the peak of the core. The other terms are heating terms. $D_{0}$ is the contribution of intrabeam scattering (via the Coulomb force) to the diffusion and has been calculated by Ruggiero ${ }^{8}$ to be $D_{0}=0.0015 \mathrm{~N}_{\bar{p}}$ $(\mathrm{eV})^{2} / \mathrm{sec}$, where $\mathbb{N}_{\bar{p}}$ is the total number of antiprotons in the Accumulator. This value of $D_{0}$ cohresponds to a momentum heating time of $2 \mathrm{hr}$. $D_{0}$ is independent of both $g$ and $E$. $D$, is the contribution of thermal noise and is proportional to $\mathrm{g}^{2}$. $\mathrm{D}_{2}$ is the Schottky heating term and is proportional to $\mathrm{s}^{2}\left(E-E_{C}\right)^{2}$. In our system $D_{1}$ is small compared with $D_{0}$. Optimum performance occurs when $g$ is adjusted so that intrabeam scattering dominates in the central part of the core and the Schottky heating term 
dominates at the edges of the core. Smaller values of $g$ leave the cooling term $F$ less than optimum ( $g=0$ means no cooling) and larger values of $g$ mean that Schottky heating is larger than the cooling. Computer calculations show that densities in excess of $1 \times 10^{5} / \mathrm{eV}$ can be reached.

The choice of $1-2 \mathrm{GHz}$ bandwidth (and $n=0.02$ ) for the tail system was made because of the desire to optimize core cooling. Since $D_{2}$ is proportional to $1 / \mathrm{n}$, a larger gain can be used to counteract intrabeam scattering in the core. Choosing a higher maximum frequency for the stack tail system would have required a lower $n$ for the lattice. This would have reduced the core cooling effectiveness.

A block diagram of the core cooling system is shown in Fig. 5-5. The zero in gain is obtained by subtracting the signals from two sets of pickups placed in a region of high momentum dispersion. One set of pickups is centered above the core energy and one is centered below. The signal is then applied to a kicker placed in a region of zero momentum dispersion. The gain profile for the core system Schottky bands at $2.2,3.0$, and 3.8 $\mathrm{GHz}$ is shown in Fig. $5-6$ abc.

5.1.6 Numerical Calculations of Momentum Cooling. A computer simulation of the combined core and stack-tail momentum cooling systems has been made. These calculations use the full theory developed by van der Meer et al. and not the simplified models given here. It has been found that a core density of $1 \times 10^{5} / \mathrm{eV}$ can be obtained after 4 hours of stacking with flux of $3 \times 10^{7} \mathrm{sec}^{-1}$. Figure 5-7 shows the stack profile as a function of time. Figure 5-8 shows the cooling term (F) including the effects of beam feedback after 3 hours. Figures $5-9$ and $5-10$ show the heating term coefficients $D \phi+D 1$ and $D 2$. Figures 5-11 and 5-12 are stability plots: the real versus the imaginary part of the cooling system gain $G$ times the beam feedback $F$. In this plot the system is stable if the curve does not enclose the point $(1,0)$. Table 5-I summarizes the parameters of the system and the results of the calculation.

5.1.7 Betatron Cooling. Betatron cooling is accomplished by using a pickup sensitive to the transverse displacement of the particles. In going from pickup to kicker the particle oscillates in betatron phase by an odd multiple of $\pi / 2$, converting the position displacement to an angle displacement. Each given particle creates a signal in the pickup which, when applied to the kicker, decreases the angle displacement. Other particles with similar revolution frequencies contribute noise that tends to increase the spread in betatron amplitudes. This situation is similar to the momentum-cooling case discussed earlier.

Betatron cooling is conventionally described in terms of the time decrease of the betatron emittance? 


$$
\frac{d \varepsilon}{d t}=\sum_{n=n}^{n} \sum_{\min } \frac{-2 G_{n+\nu} \varepsilon+\alpha G_{n+v^{2}}^{2}}{|s|^{2}}
$$

The first term in the numerator describes the effects of the Schottky signal. (In the case of betatron cooling, the heating and cooling terms may be combined into a single term as has been done in Eq. (1) above.) The signal-suppression factor $S$ is given approximately by

$$
S=1+\frac{G_{n \pm V}}{n \pm V} \psi(E) \frac{\beta^{2} E}{\eta} T
$$

The exact expression for $S$ contains, in addition to the "pole term" given above, a principal-value integral over the distribution $\psi(E)$. At the center of a symmetric distribution, the principal-value integral vanishes. Away from the center, $|s|$ tends to be smaller than $S$ at the center of the distribution. Thus, we can get approximate, but slightly pessimistic, results for betatron cooling in the core by considering only particles at the peak of the distribution. In the case of betatron cooling in the tail, the gain $G_{n \pm v}$ is sufficiently small that $S$ can be set to 1 .

The second term in the numerator of $\mathrm{Eq}$. (1) describes the heating by thermal noise. Here

$$
\alpha=2 \mathrm{TU}_{0}
$$

where $T$ is the revolution period, $U$ is the ratio of thermal noise power to Schottky signal power per particle $\varrho_{t} t i m e t=0$, and $\varepsilon_{0}$ is the emittance at $t=0$. The total thermal power is given by

$$
P_{t h}=10^{\nabla}\left(k_{B} \theta\right) B_{A}^{2} W
$$

where $v$ is the amplifier noise figure, $k_{B} \theta$ is the system temperature (in units of energy), $g_{A}$ is the amplifier gain and $W$ is the bandwidth.

The Schottky power per pickup per particle is

$$
P_{s c h}=2 e^{2} f_{0} z_{0} \frac{s^{2}}{h^{2}} \beta_{p} \varepsilon_{0} g_{A}^{2} w
$$

where $z$ is the pickup impedance, $s$ is the pickup sensitivity, $h$ is the pickup gap, $\beta$ is the $\beta$ function at the pickup. The ratio of thermal to Schottky power is 


$$
U_{0}=\frac{10^{v}\left(k_{B} \theta\right)}{2 e^{2} f_{0} z_{0}(s / h)^{2} \beta_{p} \varepsilon_{0}}
$$

The solution of Eq. (1) may be written as

$$
\varepsilon=\left(\varepsilon_{0}-\varepsilon_{\infty}\right) e^{-t / \tau}+\varepsilon_{\infty}
$$

with

$$
\varepsilon_{\infty}=U_{0} G T \varepsilon_{0}
$$

and

$$
\frac{1}{\tau}=\frac{T W}{\xi} R(\xi G)
$$

$$
\xi=\frac{\psi(E) B^{2} E}{n W}
$$

$$
R(x)=\int_{1}^{2} \frac{4 x d t}{(1+x / t)^{2}}
$$

Equations (8) through (11) have assumed that the gain $G_{n \neq v}$ is constant and equal to $G$. Equation (11) is an approximation to the sum from equation (1); the limits of integration assume an octave bandwidth. The gain $G$ is related to pickups, kickers, and amplifier gain by

$$
G=\frac{2 \sqrt{ } \beta_{k} \beta_{p}}{3 \pi} \frac{s_{p} s_{k}}{h_{p} h_{k}} \frac{e^{2} f_{o}^{2} z_{o} \sqrt{N_{p} N_{k}}}{W p} g_{A},
$$


where $\beta_{p}\left(\beta_{k}\right)$ is the betatron function at the pickup (kicker), $s_{p}\left(s_{k}\right)$ is the pickup $\mathrm{P}_{\mathrm{kicker}}$ ) sensitivity, $h_{\mathrm{p}}\left(\mathrm{h}_{\mathrm{k}}\right)$ is the gap height in the pickup (kicker), $\mathrm{N}_{\mathrm{p}}\left(\mathrm{N}_{\mathrm{k}}\right)$ is the number of pickups (kickers), $\mathrm{e}$ is the electric charge, $f_{0}=p_{1 / T}$ is the particle revolution frequency, $z_{0}$ is the pickup and kicker impedance, and $\mathrm{p}$ is the momentum.

We will build two betatron cooling systems: a stack-tail system and a core-cooling system. The stack-tail system has both pickups and kickers placed in dispersive regions and has a low sensitivity to particles in the core. The core-cooling system has both pickups and kickers placed in regions of zero dispersion and is therefore equaliy sensitive to particles in the core and tail. The gain of the core system is so low, however, that is has little effect on the tail. The core cooling system is somewhat simpler and will be discussed first.

Betatron cooling in the core. In the case of core cooling, the gain of the system is determined by the energy density of particles in the core.

The function $R$ has its maximum of 1.5 at about $x=1.5$. The gain $G$ is therefore

$$
\begin{aligned}
G & =\frac{1.5 n W}{\beta^{2} E \psi\left(E_{c}\right)} \\
& =7 \times 10^{-8},
\end{aligned}
$$

where $\psi\left(E_{0}\right)=10^{5} / \mathrm{eV}$ is the peak core density, $W=2 \mathrm{Ghz}$ (from 2 to $4 \mathrm{GHz}$ ) $\eta=.02,{ }^{\circ}{ }^{2} E=8.8 \times 10^{9} \mathrm{eV}$. Then $\varepsilon_{\infty}$ is $0.06 \pi$ mm-mrad and negligible compared to a final enlttance of $2 \pi \mathrm{mm}-m r a d$. Thus, as might have been expected, thermal noise can be neglected.

The cooling time $\tau$ is found from Eq. (g) to be 1.3 hours. Particles that have an emittance much larger than the average emittance of the core will be cooled considerably faster, however, since the cooling correction is proportional to betatron amplitude. The intrabeam scattering diffusion times have been calculated to be $9 \mathrm{hr}$ horizontally and large and negative (corresponding to slight damping) vertically. The amplifier power gain ( 90 $\mathrm{dB}$ ) and power level $(5 \mathrm{~W})$ are modest. A block diagram of the systan is shown in Fig. 5-13.

Stack-Tall Betatron Cooling. The stack tall betatron cooling system uses the same pickups as the second section of the tail momentum-cooling system. The pickup plates are centered at $-55 \mathrm{MeV}$ relative to the core. For horizontal signals the pickup is most sensitive at 1 ts edges at $-35 \mathrm{MeV}$ and $-55 \mathrm{MeV}$. Most of the cooling takes place at the $-35 \mathrm{MeV}$ edge because the momentum cooling system is pushing particles much more slowly past the -35 $\mathrm{MeV}$ edge than the $-55 \mathrm{MeV}$ edge. The pickup is most sensitive at its center ( $-55 \mathrm{MeV})$ for signals in the vertical direction. The kickers are also placed at $-55 \mathrm{MeV}$ in a region with high dispersion. A block diagram of the system is shown in Fig. 5-14. 
Approximate calculations of the system performance have been made with Eq. (1) except that the time variable has been replaced with the energy variable, using the relationship

$$
\frac{d E}{d t}=\frac{\Phi}{\psi(E)}
$$

where $\Phi$ is the flux of particles and $\psi(E)$ is the density of particles. This approximation ignores fluctuations in energy gain in the stacking process. With this approximation, Eq. (1) is easily integrated numerically. Figure 5-15 shows the emittance reduction as a function of energy. The power in each system is $50 \mathrm{~W}$.

Operation of Betatron Cooling Systems. In the Accumulator we have avallable two betatron cooling systems, elther of which is capable, or nearly capable, of cooling the beam emittance from 10 to $2 \pi \mathrm{mm}-m \mathrm{mad}$. The core system is clearly required so that a core of $\bar{p}$ 's may be held for several hours without diffusion. The tall system serves two functions: 1) to cool the betatron amplitudes in a system with a low particle density (it is not necessary to wait one half to one hour for the core cooling system to do its work) and 2) to counteract possible betatron heating by the momentum cooling system. The size of the latter effect is difficult to estimate; it depends on how well we are able to build the momentum kickers for the stack-tall system. If the effect is larger than expected, the gain of the stack-tail system can be raised to achleve better cooling, but, of course, the power requirements will be greater. Tentatively, however, we would plan to use the stack tail system to reduce the beam emittance from $10 \pi$ to $3 \pi$ or $4 \pi \mathrm{mm}-\mathrm{mrad}$ and use the core system to reduce $1 \mathrm{t}$ below $2 \pi$ mm-mrad.

5.1.8 Stochastic-Cooling Hardware. The purpose of this section is to outline some of the techniques we plan to use in the stochastic-cooling systems in the Accumulator and Debuncher rings. The engineering design of these systems is not yet complete, but they have been developed in sufficient detail to establish technical feasibility and to make concrete cost estimates. In several instances alternative technologies are available. We present here the devices that seem to be the most sound technically. If the apparently riskier solutions are in fact practical, we may be able to improve performance, cut costs, or both. In any event, a careful research and development program will be required in order for us to achieve the cooling times we expect on the basis of theoretical calculations.

The plckups we plan to use are loop couplers which consist of two parallel plate electrodes above and below (or, in some cases, to the left and right) of the beam. The electrode dimensions are shown in Fig. 5-16. A ground plane is placed above (below) the upper (lower) electrode at a distance $8_{3}$. The electrode and ground plane form the geometry of a stripline Eransmission line. The signals from the plckups are coupled to a 
printed circuit board of passive combiners as is shown in Fig. 5-17. Sixteen (or, in a few instances, 8) pickup signals are combined and brought through the vacuum tank to a preamplifier.

The impedance of the pickup is (approximately)

$$
\begin{aligned}
\mathrm{z}_{\mathrm{pu}} & =377 \frac{\mathrm{g}_{3}}{\mathrm{w}+\mathrm{g}_{2}} \Omega \\
& =94 \Omega \text { at } 1-2 \mathrm{GHz} \\
& 75 \Omega \text { at } 2-4 \mathrm{GHz},
\end{aligned}
$$

where the numbers in FIg. 5-16 have been used to calculate these impedance.

The induced current in an electrode at midband is

$$
i_{p u}=i_{b} s(x, z),
$$

where $s(x, z)$ has been calculated by Collins ${ }^{10}$ and $I_{b}$ is the beam current.

$$
\begin{aligned}
s(x, z) & =-\frac{1}{2 \pi}\left\{\operatorname{Atan} \frac{\sinh (\pi / h)\left(x+w^{\prime} / 2\right)}{\cos \pi z / h}\right. \\
& -\operatorname{Atan} \frac{\sinh (\pi / h)\left(x-w^{\prime} / 2\right)}{\cos \pi z / h} \\
& +\operatorname{Atan} \tan \frac{\pi z}{h} \tanh \frac{\pi}{h}\left(x+\frac{w^{\prime}}{2}\right) \\
& -\operatorname{Atan} \tan \frac{\pi z}{h} \tanh \frac{\pi}{h}\left(x-\frac{w^{\prime}}{2}\right)
\end{aligned}
$$

where $w^{\prime}=w+g_{2}$ is the effective plate width, $z$ is the distance above the midplane between upper and lower electrodes (+ is towards the electrode), and $x$ is the distance perpendicular to the beam direction. At $x=0$ and $z=0$ 


$$
s(0,0)=\begin{array}{ll}
0.84 & 1-2 \mathrm{GHz} \\
0.66 & 2-4 \mathrm{GHz}
\end{array}
$$

The power delivered to the preamp is thus

$P=(16$ plckups $) \times(2$ electrodes/pickup $) \times$ (current squared) $\times$ (pickup impedance)

$$
=81_{b}^{2} s^{2} z_{p u}
$$

About $0.5 \mathrm{~dB}$ of this power is lost in the process of combining signals and bringing them through the vacuum.

The pickups are placed in the vacuum tank and surrounded by liquid nitrogen. The purpose of the liquid nitrogen is twofold. It reduces the thermal noise from the terminating resistor on the combiner circuit board, and 1t allows a wider choice of materials for the printed circuit board because of the reduced outgassing at lower temperatures. A conceptual design of the system is shown in Figs. 5-18 and 5-19.

The kicker design is essentially identical to that of the pickup except that the combiner becomes a splitter for the power traveling to the electrodes. More substantial terminating resistors are also required to terminate the power, about $7 \mathrm{~W}$ per kicker for the high power stack-tail system.

The pickup signals are fed (in groups of 16) into low noise amplifiers (2.0 dB nolse figures are obtalned in commercially available amplifiers at $1-2 \mathrm{GHz}, 3.0 \mathrm{~dB}$ at $2-4 \mathrm{GHz}$ ). The signals are further combined and amplified. Provision is made following the first preamplifier for delay and amplitude adjustments. The chain of solid state amplifiers continues until a signal level of $10 \mathrm{dBm}$ is reached. The preamplification chain is shown for the stack tall system, by way of example, in Figs. 5-20 and 5-21.

The power amplification is accomplished with traveling wave tubes (TWT's). The stack tall system configuration is shown in Fig. 5-22. The system consists of a driver stage (rated at $200 \mathrm{~W}$ saturated power) followed by power output stage tubes (rated at $1 \mathrm{~kW}$ saturated power). It is necessary to run these tubes at reduced power levels in order to avoid excessive non-linear output. It is particularly important to keep the intermodulation products of the stack tail system in the frequency range of the core particles down $40 \mathrm{~dB}$, or preferably $50 \mathrm{~dB}$, from the signal level in the stack tail system. For this purpose the power output stage probably requires a filter to suppress intermodulation products.

Delay and gain adjustments are available to match the overall phase and amplitudes of the individual tubes. The output power is divided to feed groups of 16 klckers. 
STOCHASTIC-COOLING POWER

(Watts)

$\begin{array}{llc}\text { Schottky power } & \text { Tail } & \text { Core } \\ \text { Thermal power } & 900 & 10 \\ \text { Total power } & 400 & 0 \\ \text { Amplifier gain } & 1300 & 10 \\ \quad \text { Section \#1 } & 151 \mathrm{db} & 102 \mathrm{db} \\ \text { Section \#2 } & 143 \mathrm{db} & \end{array}$

\subsection{RF Stacking System}

With the injection shutter closed, antiprotons are injected from the Debuncher ring with a total momentum spread of about $0.2 \%$. The energy spread ( $18 \mathrm{MeV})$ and the Accumulator ring revolution period ( $1.59 \mu \mathrm{\mu ec})$ result in an injected longitudinal emittance of about $29 \mathrm{eV}-\mathrm{sec}$. This beam is bunched adiabatically and accelerated by $0.7 \%$ to the bottom of the tail of the previously stacked antiprotons where it is released by adiabatic debunching. Adiabatic capture can begin immediately following injection and can proceed during the time required for removal of the shutter, about 0.1 seconds.

Stability conditions resulting from the final cooled-core density set a limit on the allowable effective shunt impedance of the $\mathrm{rf}$ system to be used for stacking. This limit requires that $R / h$ be less than about $1 \mathrm{k} \Omega$. The rf voltage required to establish a given phase-space area increases linearly with the harmonic number and, because the power required is $\mathrm{V}^{2} / 2 \mathrm{R}_{\mathrm{sh}}$, the required $\mathrm{rf}$ power increases linearly, given the stability limitsh' the shunt impedance. Consequently, to minimize the cost, size, and complexity, it would appear that of stacking should be done at the lowest possible harmonic number. On the other hand, very low harmonic numbers and very low voltages result in excessively long synchrotron periods that are not consistent with the rapid production cycling rate. An adequate compromise appears to be $\mathrm{h}=10,6.289 \mathrm{MHz}$. The parameters associated with $\mathrm{rf}$ stacking at $h=10$ are listed in Table 5-II.

With a phase-oscillation period of $10 \mathrm{msec}$ and a deceleration time of 30 msec, the entire operation of establishing a moving bucket, deceleration, and adiabatic debunching can be accomplished within 0.1 seconds after the beam is infected.

At frequencies well below the GHz cooling band, the cooled core may be subject to longitudinal instabilities induced by the shunt impedance of the stacking rf cavity or other similar device. The stability criterion resulting from various dispersion analyses can be written in a limiting form 


$$
\left|\frac{Z}{n}\right| \leq F \frac{(\Delta E / E)^{2} E_{s} \eta}{I},
$$

where the form factor $F$ is about unity for a conservative estimate of the stability of a roughly Gaussian distribution and $\Delta E$ is the full width at half maximum.

The cooled core has the parameters
$\triangle \mathrm{p} \quad$ FWHM
4. $1 \mathrm{MeV} / \mathrm{c}$
N (antiprotons)
$4.3 \times 10^{11}$
I
$4.32 \times 10^{-2}$ Amperes
$\eta$
0.02

With these parameters the critical value of $\mathrm{Z} / \mathrm{n}$ is about 900 ohms. The shunt impedance of the bunching cavity is in agreement with this stability requirement for harmonics of interest. 
Table 5-II. RF STACKING PARAMETERS

Injected Longitudinal Emittance

Stacking $\mathbf{r f}$ Total Bucket Area

Stationary Bucket RF Voltage

Stationary Bucket Phase Oscillation Period

$\Delta p / p$ Required for stacking

$\Delta(\mathrm{cp})$

Acceleration Synchronous Phase Angle

$\Gamma=\sin \phi$

Moving Bucket Factor $\alpha(\Gamma)$

RF Voltage During Acceleration

Time Required for Acceleration

Number of Accelerating Cavities

Cavity Structure

Cavity Small Signal Shunt Impedance

Peak RF Power Requirement

Average RF Power Requirement (Cooling, etc)

Harmonic Number

RF Starting Frequency

$\eta=\gamma_{t}{ }^{-2}-\gamma^{-2}$

B

Injection Orbit Circumference

Stacking Efficiency*
$29 \mathrm{eV} \mathrm{sec}$

$30 \mathrm{eV} \mathrm{sec}$

$7.8 \mathrm{keV}$

$9.4 \mathrm{msec}$

$0.7 \%$

$62.2 \mathrm{MeV}$

10 degrees

0.1736

0.6964

$16.1 \mathrm{kV}$

30.2 msec

1

40 NiZn Ferrite Cores

$12 \mathrm{k} \Omega$

$10 \mathrm{~kW}$

$1 \mathrm{~kW}$

10

$6.28695 \mathrm{MHz}$

0.02

0.99447

$474.202 \mathrm{~m}$

$98 \%$

* Based on computer simulation 


\subsection{Accumulator Lattice}

The lattice of the Accumulator has been designed to accept the injection of antiprotons every few seconds at an energy of $8 \mathrm{GeV}$, momentum stack and stochastically cool the antiprotons, accumulate over a period of several hours a very dense core of antiprotons, and, finally, extract a high-intensity beam to re-inject into the Main Ring and Tevatron. The requirements on the lattice are twofold. The Accumulator must be a high-class storage ring capable of rellable operation, and it must accommodate all the conditions imposed by the stochastic cooling systems. The second set of requirements has led to the general design of this ring and Its somewhat unusual appearance, while those of the first set have also been incorporated. These general conditions are listed below:

(1) The momentum mixing factor must be correct.

$$
\left|1 / Y_{T}^{2}-1 / Y^{2}\right|=0.02
$$

where $Y_{r}$ is the transition gamma of the ring and $Y$ is the relativistic gamma factor of the particle. Thus for $8-\mathrm{GeV}$ kinetic energy the ring must have

$$
Y_{T}=5.7 \text { or } 10.5
$$

(11) There must be several long straight sections, some 16 meters long, with very small transverse beam sizes. Some of these must have zero dispersion and the rest dispersions of $\sim 10 \mathrm{~m}$.

(1i1) Betatron cooling straight sections must be an odd-multiple of $\pi / 2$ apart in betatron phase. Plckup and kicker straight sections must be far enough apart physically so that a chord will be at least 75 nsec shorter than the arc for signal-transfer purposes.

(iv) The $B$ values of the lattice should be about the same for the horizontal and vertical planes in the straight sections.

(v) The ring should match the booster circumference.

(vi) There should be easy injection and extraction schemes.

(vi1) The beam everywhere should be as small as possible, consistent with the large-dispersion straight sections.

(vi1i) The ring must have very good chromaticity corrections, be situated far from any resonances, have tuning flexibility, and generally be a good storage ring. 
The result of all of the above criteria is shown on Fig. 5-23.. The ring is a six-sided figure of periodicity three, having three 16-meter long zero-dispersion straight sections and three 16-meter long high-dispersion straight sections. All the straight sections have a minimum beta-function of $7.5 \mathrm{~m}$ in each plane, giving the smallest average $B$ possible for the length of the straight section. The structure and lattice parameters of this ring are listed in Table 5-III and the lattice functions for one third of the ring are plotted in Fig. 5-24. Horizontal and vertical aperture requirements are plotted in Figs. 5-25 and 5-26. Figure 5-25 shows four beams, the injected beam, the injected orbit, the stacking orbit, and the accumulated stack. The layout of a period is shown in Fig. 5-27.

Injection into and extraction from this machine are done in a similar manner. The description given will be for injection. The injection orbit is displaced radially inward by approximately $0.7 \%$ in $\Delta p / p$. The shuttered kicker in the high-dispersion straight section is closed just before the $\bar{D}$ 's are injected. The momentum displacement is enough to allow the injected beam to clear the shutter. Beam is transferred from the Debuncher ring via the 3.8-m long, 8-kG current septum magnet located at the upstream end of a zero-dispersion straight section. It arrives at the kicker and is kicked onto the injection orbit. The kicker is $2 \mathrm{~m}$ long and has a field of $500 \mathrm{G}$. The shutter is then opened and the beam is rf stacked. A plot of the injection-extraction orbit is shown in Fig. 5-28. The injection and extraction positions are shown in Fig. 5-23. 
Table 5-III. ACCUMULATOR PARAMETERS

1. General

$\begin{array}{lc}\text { Energy } & 8.0 \mathrm{GeV} \\ \text { Bend field } & 16.98 \mathrm{kG} \\ \text { Magnetic bend radius ( }) & 17.46 \mathrm{~m} \\ \text { Radius } & 75.45 \mathrm{~m} \\ \text { Revolution time } & 1.59 \mu \mathrm{sec} \\ \text { Superperiodicity } & 3 \\ \text { Focusing structure } & \text { Separated function } \\ & \text { FODO nomal cell }\end{array}$

Nominal working point

$$
\begin{array}{ll}
v_{x} & 6.74 \\
v_{y} & 8.24
\end{array}
$$

Nominal chromaticity

$$
\begin{array}{lr}
\xi_{\mathrm{x}} & -9.23 \\
\xi_{\mathrm{y}} & -11.86
\end{array}
$$

Transition and mixing factor

$$
\begin{array}{cc}
Y_{\mathrm{T}} & 5.76 \\
1 / Y_{\mathrm{T}}{ }^{2}-1 / \mathrm{Y}^{2} & .019
\end{array}
$$

\section{Magnets}

Number of dipoles

$$
\begin{array}{r}
18-15.0 \mathrm{ft} \\
6-10.0 \mathrm{ft} \\
6-5.0 \mathrm{ft}
\end{array}
$$

Number of quadrupoles (*)

$$
\begin{array}{r}
12-22.53 \text { in. } \\
12-50.17 \text { in. } \\
12-27.65 \text { in. } \\
12-18.00 \text { in. } \\
6=20.83 \text { in. } \\
6-31.37 \text { in. } \\
6=29.46 \text { in. } \\
6-28.94 \text { in. } \\
6-6.79 \text { in. }
\end{array}
$$

Quadrupole gradients $\quad \pm 97.85 \mathrm{kG} / \mathrm{m}$ 
(*) Note: For this design, all quadrupoles run at the same gradient. The actual machine will not have so many different length quadrupoles.

3. Structure

A. Dipoles

Length

$\begin{array}{lr}\text { (B1) } & 5 \mathrm{ft} \\ \text { (B2) } & 10 \mathrm{ft} \\ \text { (B3) } & 15 \mathrm{ft}\end{array}$

Quadrupoles

$\begin{array}{lr}\text { (QF1) } & 22.5 \mathrm{in.} \\ \text { (QD2) } & 50.2 \mathrm{in.} \\ \text { (QF3) } & 27.7 \mathrm{in.} \\ \text { (QF4) } & 20.8 \mathrm{in.} \\ \text { (QD5) } & 31.4 \mathrm{in.} \\ \text { (QF6) } & 29.5 \mathrm{in} . \\ \text { (QD7) } & 28.9 \mathrm{in} . \\ \text { (QF8) } & 18.0 \mathrm{in} . \\ \text { (QD9) } & 18.0 \mathrm{in.} \\ \text { (QF 10) } & 6.8 \mathrm{in} . \\ \text { (QF11) } & 27.7 \mathrm{in} . \\ \text { (QD12) } & 50.2 \mathrm{in} . \\ \text { (QF13) } & 22.5 \mathrm{in} .\end{array}$

C. Straight sections

$\begin{array}{rr}\text { LS } & 315.0 \mathrm{in.} \\ 0 & 20.0 \mathrm{in.} \\ 00 & 48.0 \mathrm{in} . \\ 01 & 40.0 \mathrm{in.} \\ 02 & 35.6 \mathrm{in} . \\ 03 & 251.5 \mathrm{in} . \\ 04 & 127.9 \mathrm{in} . \\ 05 & 289.4 \mathrm{in} . \\ 06 & 162.9 \mathrm{in} . \\ 07 & 174.4 \mathrm{in.} \\ 08 & 69.0 \mathrm{in} .\end{array}$

D. Sextant structure (S)

$$
\begin{aligned}
& \begin{array}{lllllllllll}
\mathrm{LS} & (\mathrm{qf} 1) & 0 & \text { (QD2) } & 01 & \text { (QF3) } & 02 & \text { (B1) } & 03 \text { (QF4) }
\end{array} \\
& 04 \text { (QD5) } 05 \text { (QF6) } 06 \text { (QD7) } 07 \quad 0 \text { (B2) } 0 \text { (QF8) } \\
& 00 \text { (B3) } 0 \text { (QD9) } 00 \text { (B3) } 0 \begin{array}{lllllllll} 
& 0 & \text { (QF 10) } & 0
\end{array} \\
& 08 \text { (QF11) } 01 \text { (QD 12) } 0 \text { (QF13) LS }
\end{aligned}
$$

$$
S(S) S(S) S(S)
$$




$$
\begin{array}{lr}
\text { Length of central orbit } & 474.0918 \mathrm{~m} \\
& 1555.42 \mathrm{ft}
\end{array}
$$

4. Aperture and Acceptance

\begin{tabular}{|c|c|c|c|}
\hline & Maxima & In Dipole & LS1 \\
\hline$\frac{x}{y}$ & $\begin{array}{r}31.30 \\
30.20 \\
9.36\end{array}$ & $\begin{array}{r}28.77 \\
18.37 \\
8.04\end{array}$ & $\begin{array}{l}\overline{7.51} \\
7.55 \\
0.02\end{array}$ \\
\hline
\end{tabular}

$$
\text { Lattice functions }
$$

Required beam

$$
\begin{aligned}
& \text { emittance } \\
& \text { momentum aperture }
\end{aligned}
$$

$$
\begin{aligned}
& \varepsilon_{x}=\varepsilon_{y}=10 \pi \mathrm{mm}-\mathrm{mrad} \\
& \Delta \mathrm{p} / \mathrm{p}_{\text {total }}=2.30 \%
\end{aligned}
$$

Beam sizes (*)

$\begin{array}{lll}\mathrm{A}_{\mathrm{x}} \text { (full) } & \frac{\text { Dipole }}{208.0 \mathrm{~mm}} & \frac{\text { Quadrupole }}{246.0 \mathrm{~mm}} \\ \mathrm{~A}_{\mathrm{y}} & 27.1 & 34.7\end{array}$

(*) Note: Beam sizes given are the largest apertures. In most magnet3, beams are significantly smaller. 


\subsection{Accumulator Vacuum System}

5.4.1 Vacuum Requirements. The base pressure is determined by the effects of the residual gas on the accumulated antiproton beam. These effects include particle loss by single Coulomb scattering and nuclear interactions, beam heating by multiple Coulomb scattering, energy loss by Ionization, and effects of neutralization by positive ions attracted to the negative beam.

A detalled analysis has been made ${ }^{11}$ of these effects. As a result, the system is designed for a nominal pressure of $3 \times 10^{-10}$ Torr (nitrogen equivalent). At this pressure, the single-scattering lifetime will be 240 $\mathrm{hr}$ and the nuclear-interaction 11 fetime will be $2000 \mathrm{hr}$. Thus these effects are negligible. The heating rate for the final stack from multiple scattering will be $2 \times 10^{-5} / \mathrm{sec}, 10$ times less than the cooling rate. (Here a gas composition of $50 \% \mathrm{H}_{2}, 50 \% \mathrm{~N}_{2}$ or $\mathrm{CO}$ is assumed). The energy loss per antiproton will be $20 \mathrm{keV} / \mathrm{hr}$. Both the heating and energy loss can be easily compensated by the stochastic-cooling systems. We will keep the nominal design value of the neutralization factor $H<0.03$. With this value, the scattering by positive lons trapped in the beam will be increased less than 2\%. The vertical space-charge tune shift will be reduced by $10^{-3}$. Thus these neutralization effects are negligible.

5.4.2 Vacuum system layout and characteristics. The pressure requirement can be met with sputter ion-pumps. Furthermore vacuum-annealed austenitic high-tensile stainless steel will be used for the chambers so that specific degassing rates of better than $1 \times 10^{-12}$ Torr- $\ell / \mathrm{cm}^{-2}-\mathrm{sec}$ can be attained. The maximum design bakeout temperature is $300^{\circ} \mathrm{C}$, with $200^{\circ} \mathrm{C}$ permissible in difficult places like the dipole vacuum chamber.

Figure 5-29 shows the vacuum-system layout over one-sixth of the Accumulator ring. The sputter ion pumps should have a speed of $200 \mathrm{l} / \mathrm{sec}$. There will be six sector valves, placed near the Q1 locations, where the beam sizes are small.

The baking equipment (heaters, controls, themal insulation) will be installed in a permanent fashion to allow bakeout to proceed without major preparations. Exceptions are areas that cannot tolerate the high temperature; e.g., pump magnets, cable feedthroughs and special devices.

Pump-down during bakeout wlil be carrled out using mobile turbopump sets with Ilquid-nitrogen cold traps. This will allow the use of a large number of pumps in any given section being baked. These carts will be connected to metal valves distributed for that purpose throughout the system. Tests indicate that the pressure during bakeout has little effect on the success of the bake.

Vacuum gauges include 6 Pirani gauges to monitor pumpdown, 36 Bayard-Alpert ionization gauges, and 6 mass-analyzer heads, located near areas of complex equipment to monitor leaks and contamination. 
The arrangement has not been completely specifled for the straight sections, where speclal equipment will be located. Some of the equipment may contain epoxy (one proposed cooling pick-up design contains printed-circuit boards), which would require cooling to at least liquid nitrogen temperature to suppress contamination of the rest of the system, and would need isolation valves that close automatically on warm up.

Clearing electrodes will be installed to remove low-energy positive ions and thus keep the neutralization factor $H<0.03$. There will be a pair of electrodes at the downstream end of each magnet. Ions move longitudinally to the electrodes by $E \times B$ drifting caused by the beam electric field and the ring guide field. This kind of system has been used successfully in the ISR.

The straight sections between magnets will also need clearing electrodes to avoid trapping lons in the cool-beam potential of $5 \mathrm{~V}$. We plan to apply a de potential of more than $5 \mathrm{~V}$ to the clearing electrodes and to the beam-position detector electrodes whenever these are in $a$ suitable location.

All devices and ring sections will undergo a preliminary bake and low-pressure test before being installed. Their design will conform with strict rules of choice of material and will be subject approval by the vacuum coordinator.

The vacuum control system will be constructed along the design evolved for the Tevatron. It is highly modular and economical. Most of the required modules and device controls have already been developed, including ion pump supplies and ion gauge controllers. A card cage containing all control modules interfaces to the host computer through a CAMAC module. Much of the necessary software can be used or adapted.

\subsection{Accumulator Magnets}

The main magnet system consists of 30 dipoles and 78 quadrupoles. In Table 5-IV is shown a list of magnets, lengths, strengths and required apertures. The apertures shown assume construction tolerances of $2.5 \times 10^{-4}$ (relative standard deviation) for dipole strengths, dipole level angles (radians), and quadrupole randon position errors. Sufficient space is allowed for at least $4 \sigma$, where $\sigma$ is the standard deviation of the expected orbit position error, or the beam emittance plus momentum spread plus $10 \mathrm{~mm}$ whichever is larger. In addition, space is left for $4 \mathrm{~mm}$ (thickness plus deflection) vacuum chamber thickness and $5 \mathrm{~mm}$ insulation thickness. Several mm must be included also for copper heat transporters to bake the chamber (dipoles) with heaters on the edges.

There are three lengths of dipoles, all with field strengths of $1.698 \mathrm{~T}$. Their properties are described in Table 5-V, assuming all have the same aperture. Since only one dipole needs more than $130 \mathrm{~mm}$ radial aperture, further reflection may lead to two dipole profiles. Because of 
the large sagitta, it is expected that these magnets will be curved. The coils can be made of four pancakes plus single-layer saddle coils.

There are 13 different quadrupoles, of two different profiles. Q1-Q9 have poletip radii of $43.3 \mathrm{~mm}$ and 8 different lengths. Q10-Q13 have poletip radii of $84.1 \mathrm{~mm}$ and four different lengths. The properties of these quadrupoles are shown in Table 5-VI. The smaller quadrupoles can be run on the same bus with the larger quadrupoles by putting shunts in parallel in each coll package. Each quadrupole will have a trim winding with capability of about $10 \%$ of the quadrupole strength. The coils are fabricated in individual layers and assembled on the quad half cores, which are then assembled as a complete magnet. All quadrupoles have the same gradient, $9.7845 \mathrm{~T} / \mathrm{m}$. 
Table 5-IV. ACCUMULATOR RING MAGNETS

Magnet Type

Q1

Q2

Q3

B1

Q4

Q5

Q6

Q7

B2

Q8

B3

Q9

B3

Q10

B3

Q11

Q12

Q13
Strength

$\underset{n}{9.7845 \mathrm{~T} / \mathrm{m}}$

$n$

$1.698 \mathrm{~T}$

$9.7845 \mathrm{~T} / \mathrm{m}$

"

"

$"$

$1.698 \mathrm{~T}$

$9.7845 \mathrm{~T} / \mathrm{m}$

$1.698 \mathrm{~T}$

$9.7845 \mathrm{~T} / \mathrm{m}$

$1.698 \mathrm{~T}$

$9.7845 \mathrm{~T} / \mathrm{m}$

$1.698 \mathrm{~T}$

$9.7845 \mathrm{~T} / \mathrm{m}$

"

n
Arc Length (m)

0.572

1.274

0.702

1.524

0.529

0.797

0.748

0.735

3.048

0.457

4.572

0.457

4.572

0.178

4.572

0.702

1.274

0.572
Aperture VXH

or Poletip

Radius (mm)

43.274

"

$50 \times 225$

43.274

$"$

"

"

$50 \times 225$

43.274

$50 \times 225$

43.274

$50 \times 225$

84.109

$50 \times 225$

84.109

"

$"$ 
Table 5-V. DIPOLES

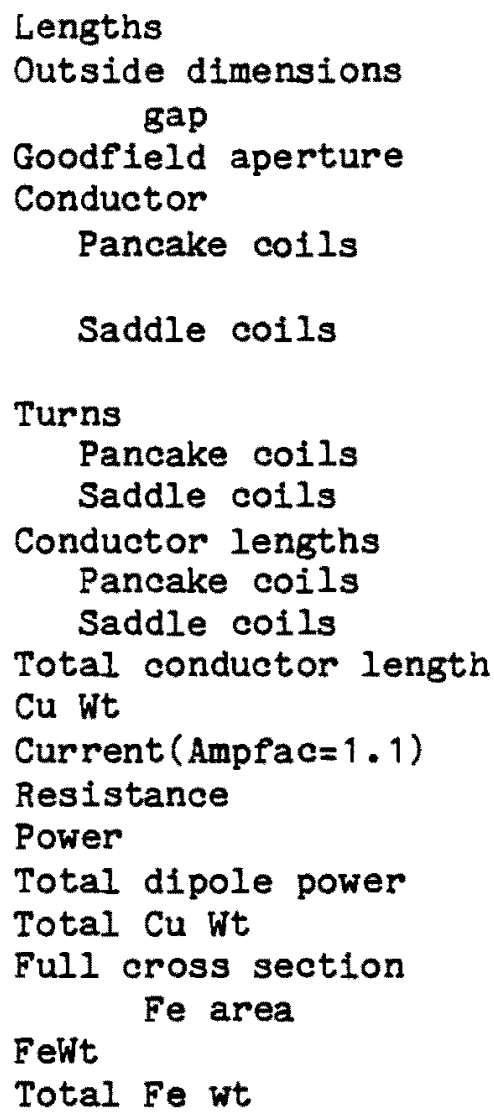

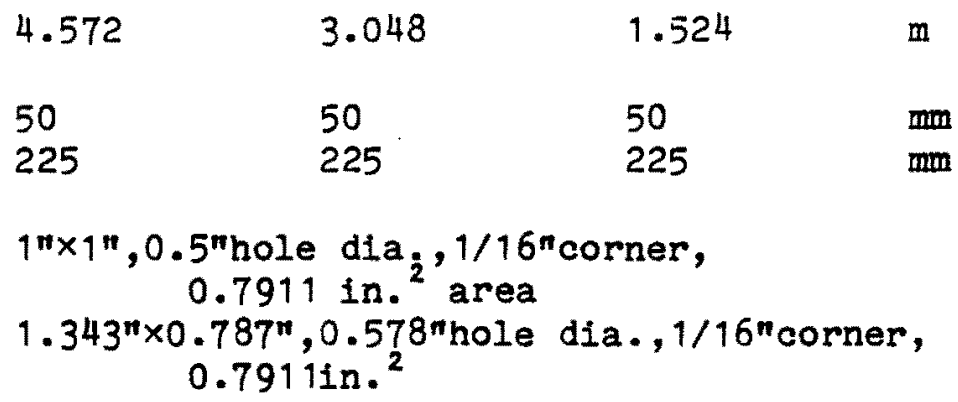

40

10

409.5

114

523.5

5,234

1.486

19.1

42.1

795

98.8

0.557

287.5

83.5

371

3,710

1.486

15.5

29.9

165.6

63

228.6

2,288

1.486

8.3

18.4

49.2

0.886 $\frac{m}{m m}$ 
Table 5-VI. QUADRUPOLES

\section{Lengths}

Poletip radius

Conductor

Total conductor length (all magnets)

Cu Wt

Current

Resistance

(all magnets)

Power

(all large quads)

Full cross section (Fe area)

Fewt

0.178 to 1.274
total 16.3
84.1
0.82 square
24,228
35,565
$810 \mathrm{~A}$
0.442
290
0.476
134.5

0.457 to 1.274

total 37.6

43.27

0.4096 square

47,083

27,344

$202.5 \mathrm{~A}$

3.354

$137 \cdot 7$

0.183

119.4 m

m

mm

in.

ft

16

A

$\Omega$

$\mathrm{kW}$

$m^{2}$

$10^{3} 1 b$ 
REFERENCES

1. D. Möhl, G. Petrucci, L. Thorndahl, and S. van der Meer, Physics Reports C, 58 (1980) 73-119.

2. S. van der Meer, "Stochastic Stacking in the Antiproton Accumulator," CERN/PS/AA/78-22 (1978); unpublished.

3. A.G. Ruggiero, "Stochastic Cooling - A Comparison with Bandwidth and Lattice Functions," Fermllab ̄̄ note 171 (1981), unpublished.

4. A.G. Ruggiero, "Pickup Loop Analysis" $\bar{p}$ note 148 (1981), unpublished.

5. S. van der Meer, "A Different Formulation of the Longitudinal and Transverse Beam Response," CERN/PS/AA/80-4 (1980), unpublished.

6. A.G. Ruggiero, "Theory of Signal Suppression for Stochastic Cooling with Multiple Systems," Fermilab $\bar{p}$ note 193 (1982), unpublished.

7. F. Sacherer, "Stochastic Cooling Theory," CERN/ISR/TH/78-11 (1978), unpublished.

8. A.G. Ruggiero, "Revised Intrabeam Scattering Calculation," Fermilab p note 192 (1982), unpublished.

9. This discussion follows the discussion of C. Kin, "Design Options for the Fast. Betatron Precooling systems in the Debuncher or in the Injection Orbit," LBL Note BECON-25, unpublished.

10. T. Collins, unpublished.

11. A.G. Ruggiero, "Vacuum Considerations for the Accumulatior Ring," Fermilab $\bar{p}$ Note 194 (1982), unpublished. 


\section{EXTRACTION OF ANTIPROTONS FROM THE}

ACCUMULATOR CORE AND ACCELERATION TO 1 TEV

\subsection{Extraction}

6.1.1 Introduction. The number of $\bar{p}$ 's that need to be extracted from the core depends on the Iuminosity and the number of bunches that are needed for colliding-beams experiments. For example, if only one experiment is running at a luminosity of less than $10^{29} \mathrm{~cm}^{-2} \mathrm{sec}^{-1}$, a single bunch of $3 \times 10^{10}$ antiprotons and a single bunch of protons stored in the Energy Saver ring will be sufficient. A more typical mode of operation would require three bunches of antiprotons, each containing $10^{11}$ particles, and a similar number of proton bunches. Before the desired number of antiprotons are transferred to the Energy Saver ring, it is prudent to transfer a single low-intensity bunch of perhaps $3 \times 10^{9}$ particles to check the proper functioning of the transfer sequence. The $r f$ system that extracts particles from the core of the stack must be capable of a number of modes of operation in order to fulfill the preceding requirements. Since the most flexible and reliable method of transfer is a single-bunch transfer, it is adopted. The $\bar{p}$ 's are removed from the core in the appropriate quantity with optimum longitudinal emittance by adiabatic capture in a single bucket followed by $\mathrm{rf}$ unstacking. The $\overrightarrow{\mathrm{p}}$ distribution just prior to this operation will be displayed as a schottky spectrum and a computer-assisted choice will be made of the frequency and bucket area that are required. Each extraction will result in a single $\bar{p}$ bunch injected into the Tevatron. $\overline{\mathrm{p}}$ bunches are to be stored in the Tevatron at $150 \mathrm{GeV}$ until the required number, which will be between 1 and 6 , has been reached.

A group of proton bunches will also be placed in the Tevatron at the correct azimuthal locations. We plan to build the system with enough flexibility to inject either protons or antiprotons first, using operational experience to optimize this process. The entire ensemble will then be accelerated to the operating energy. The of cavities in the Tevatron are located and phased in such a way as to provide essentially orthogonal rf control of the two counterrotating beams.

A complete numerical example of the scenario described above will be presented in this section. Slight changes in the specific parameters selected can be expected to occur as the oyer-all design progresses.

6.1.2 Accumulator Beam Manipulation. Prior to extraction of the first $\bar{p}$ bunch, a nearly Gaussian distribution of $\bar{p}$ 's will exist in the core. The density can be characterized by two numbers, the total number of $\bar{p}$ 's in the

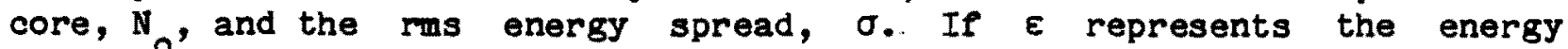
difference between the particle energy and the most probable energy of the particles in the core, then the density function $\psi(\varepsilon)$ can be written as 


$$
\psi(\varepsilon)=\frac{\mathrm{N}_{\odot}}{(2 \pi)^{1 / 2} \sigma} \exp \left(-\frac{\varepsilon^{2}}{2 \sigma^{2}}\right)
$$

The example presented here uses the following values for these parameters; $\sigma=2.0 \mathrm{MeV}, \mathrm{N}_{0}=4.5 \times 10^{11}$ and $\psi(0)=10^{5} \mathrm{eV}^{-1}$. Because we want to extract an ensemble with the smallest longitudinal emittance, we begin the adiabatic capture with a bucket centered at the peak of the distribution. We plan to extract a single bunch containing $8 \times 10^{10} \bar{p}^{\prime} \mathrm{s}$. These $\bar{p}^{\prime} \mathrm{s}$ exist within a range of $\pm 452.5 \mathrm{keV}$. The revolution period of the ring is 1.59 usec, so the longitudinal emittance of the core region selected is 1.44 eV-sec. The accumulator parameters which are used in this calculation are $\bar{R}=75.4716 \mathrm{~m}, \Pi=0.02, E=8.938 \mathrm{GeV}$, and $\Delta \mathrm{p} / \mathrm{p}=1.6 \%(\Delta(\Delta \mathrm{p})=142$ $\mathrm{MeV} / \mathrm{C}$ ) from core to extraction orbit.

The $r f$ voltage required to develop a single bucket of area $1.44 \mathrm{eV}-\mathrm{sec}$ at harmonic $\mathrm{h}$ is $\mathrm{V}=1.78 \mathrm{~h}^{3}$. At $\mathrm{h}=1$, only $1.78 \mathrm{~V}$ is required and it may be difflcult to establish such a low voltage with sufficient precision to control accurately the number of $\bar{p}$ 's extracted. We propose to extract using a single $h=2$ bucket with the remaining bucket suppressed. The required $\mathrm{rf}$ voltage is $14.24 \mathrm{~V}$. Following adiabatic capture, a moving bucket will be established for deceleration to the extraction orbit.

The time required for this unstacking process depends upon the choice of $r f$ voltage and synchronous phase angle. In order to minimize both the disturbance of the stacked $\bar{p} ' s$ and the particle loss from the moving bucket, the bucket will be moved very slowly with a small phase angle. After the first $\bar{p}$ bunch has been extracted and placed in the Tevatron at $150 \mathrm{GeV}$, the duration that this bunch is required to reside in the Tevatron at low field depends upon the time required to extract the remaining bunches from the core. If the lifetime at $150 \mathrm{GeV}$ is not long enough, it may be necessary to accelerate the process. Optimization of this time can only be accomplished after the Energy Saver and the Antiproton Source is operational. The rf voltage required to maintain the specified bucket area, the deceleration rate, and the time required for deceleration to the extraction orbit are shown in Table $6-I$ for several synchronous phase angles.

Table 6-I. RF PARAMETERS FOR DECELERATION TO THE EXTRACTION ORBIT (CONSTANT BUCKET AREA 1.44 EV-SEC.)

\begin{tabular}{|c|c|c|c|c|c|}
\hline$\left.\phi_{\mathrm{g}}\right)$ & $\Gamma=\sin \phi_{s}$ & $\alpha(\Gamma)$ & $v_{r f}($ volts $)$ & $\begin{array}{l}\text { Decel.Rate } \\
(\mathrm{MeV} / \mathrm{sec})\end{array}$ & $\begin{array}{c}\text { Decel. Time } \\
(\mathrm{sec})\end{array}$ \\
\hline $\begin{array}{l}10 \\
20 \\
30\end{array}$ & $\begin{array}{l}0.1736 \\
0.3420 \\
0.500\end{array}$ & $\begin{array}{l}0.696 \\
0.4918 \\
0.3334\end{array}$ & $\begin{array}{l}30 \\
59.8 \\
130\end{array}$ & $\begin{array}{r}3.33 \\
12.93 \\
41.1\end{array}$ & $\begin{array}{l}43 \\
11 \\
3.5\end{array}$ \\
\hline
\end{tabular}


Frequency and phase control of the rf system during unstacking may be improved by a phase-lock system that partially locks the system to the coherent component of beam current. Since this component of current is of order $10 \mathrm{~mA}$, detection and phase locking should not be technically difficult. The bunch length during the unstacking process will be about 500 nsec.

When the bunch reaches the extraction orbit, the rf voltage is raised to $100 \mathrm{~V}$, resulting in a total bunch length of 377 nsec or about 112 meters. Since the total momentum spread $\Delta p / p$ of this bunch is $4.5 \times 10^{-4}$, it can easily be injected into the Main Ring. The Accumulator of frequency is $628.695 \mathrm{kHz}$. The extraction kicker shutter is closed, isolating the bunch from the remaining accumulated beam, and the single bunch is extracted and injected into the Main Ring.

Movement of the extraction bucket through part of the stack is a form of displacement acceleration resulting in partial replacement of some of the $\bar{p}$ density in the core. Moreover, the cooling system will very quickly fill the depleted region left by the extracted bunch. During subsequent acceleration of the extracted bunch to $150 \mathrm{GeV}$ and prior to the next extraction, core cooling systems will re-establish a $\bar{p}$ density in the core which is adequate for extraction.

The single-bucket rf wave consists of one complete sinusoidal wave with a period of one-half the rotation period. Because the fundamental frequency is $0.632 \mathrm{MHz}$, such a wave can easily be generated with Fourier components below $100 \mathrm{MHz}$. The accelerating structure may consist of an insulating gap in the beam pipe in parallel with a $50-\Omega$ resistance of sufficient power-disslpating capability. This structure will be contained within a shielded enclosure with sufficiently high shunt inductance (introduced by high-permeability ferrite) so that the load presented to a broad-band amplifier will be essentially real over the operating range. The power requirements will be less than $100 \mathrm{~W}$. The $50-\Omega$ real 1mpedance presented to the beam by this structure is well within the longitudinal-stability impedance limit.

\subsection{Acceleration}

6.2.1 Introduction. We describe here the management of a single 1.5 eV-sec p bunch in the Main Ring. The Main Ring is not capable of accelerating such a large emittance in a single bunch without excessive particle loss. For this reason, the bunch is broken up into several (5 or 7) adjacent bunches, accelerated to $150 \mathrm{GeV}$, and reconstituted into a single bunch at $150 \mathrm{GeV}$.

6.2.2 Injection and Rebunching. The $\overline{\mathrm{p}}$ bunch, about $377 \mathrm{nsec}$ in length, is injected into a matched bucket in the Main Ring at $h=53$. The erequency is $2.5148 \mathrm{MHz}$ and the bucket length, or period, is $397.6 \mathrm{nsec}$. By adjusting 
the phase of the Main Ring $r f$ at a particular time, the $\bar{p}$ bunch can be extracted at a selected turn and injected accurately into the Main Ring bucket. The required bucket height is $2.53 \mathrm{MeV}$, requiring only 477 volts at $2.5148 \mathrm{MHz}$.

After injection, the $h=53 \mathrm{rf}$ voltage is raised adiabatically to 15.5 $\mathrm{kV}$ so that the bunch length shrinks to 132.5 nsec, which spans exactly 7 'normal' $\mathrm{h}=1113 \mathrm{rf}$ periods in the Main Ring. With the bunch held at this length by the $h=53 \mathrm{rf}$ voltage, the $h=1113,52.812 \mathrm{MHz}$ voltage is raised adiabatically so that the bunch is rebunched into seven bunches. The bunch with the largest area, located at the center of the ensemble of bunches, has a longitudinal emittance of about $0.2 \mathrm{eV}-\mathrm{sec}$. This can be accelerated easily in the Main Ring. This rebunching process is shown in Figs. 6-1 through 6-3 for a biGaussian distribution in the $h=53$ bucket. After capture in $\mathrm{h}=1113$ buckets, the ensemble of seven adjacent bunches is accelerated to $150 \mathrm{GeV}$. The longitudinal phase space area is expected to increase by a factor 1.5 during acceleration.

6.2.3 Bunch Recombination at $150 \mathrm{GeV}$. At $150 \mathrm{GeV}$, the Main Ring field is held on flat-top while the seven adjacent bunches are coalesced into a single bunch. The $h=1113 \mathrm{rf}$ voltage is reduced slowly over a period of 0.1 seconds to about $6 \mathrm{kV}$, at which point the center bucket is completely full and the entire ensemble is reduced to the smallest practical momentum spread. At this point, the $h=53 \mathrm{rf}$ system is turned on at a level of about $25 \mathrm{kV}$ while the $\mathrm{h}=1113$ voltage is removed. The low-momentum $\overline{\mathrm{p}}$ bunch, which extends over $7 / 21$ (or $\pm \pi / 3$ ) of the $h=53$ bucket, now begins a coherent synchrotron motion into a roughly vertical strip. Since the charge does not extend far into the bucket, this motion is quite linear. After slightly more than one-quarter of a synchrotron-oscillation (about $0.11 \mathrm{sec}$ or 5200 turns), the $\mathrm{h}=53$ voltage is removed and the vertical distribution is recaptured in an $h=1113$ bucket of a size chosen to match accurately the rotated distribution. The re-applied voltage will create a bucket height of about $125 \mathrm{MeV}$ and the $\vec{p}^{\prime} s$ (or p's) are expected to fill this bucket, so the final $\bar{p}$ bunch longitudinal emittance is as large as $3 \mathrm{eV}-\mathrm{sec}$. The $\mathrm{h}=1113 \mathrm{rf}$ voltage required for this is $500 \mathrm{kV}$. A computer model of this process is shown in Fig. 6-4.

In Fig. 6-5, we show the results of a preliminary experiment in bunch coalescing. The experiment is done at $h=159$ using four adjacent proton bunches. The successive oscilloscope traces starting at the bottom of the pictures show two or four proton bunches merging into a single bunch of larger intensity and emittance, as expected. Since the bucket covered only seven $h=1113$ bucket lengths, the four bunches extended farther into the bucket than is proposed. This results in a more nonlinear process than will occur in the proposed scheme.

The same sequence could also be done with an ensemble of five, or even three, bunches. It would result in a more linear coalescing process at the possible expense of more dilution during acceleration, since each bunch would have a larger longitudinal emittance than in the case of seven 
bunches. The optimum number of bunches to be accelerated will be determined experimentally when the commissioning begins.

Following recapture, the $r f$ voltage is raised to $1 \mathrm{MV}$. As a result, the bunch length shrinks to $12 \mathrm{nsec}$ full width, and the bunch height grows to $\pm 166 \mathrm{MeV}$, corresponding to a momentum spread $\Delta \mathrm{p} / \mathrm{p}$ of $\pm 1.1 \times 10^{-3}$. The bunch parameters are ideal for injection into pre-established matching buckets in the Tevatron.

6.2.4 Transfer of Beam from Main Ring to Tevatron. Both the forward $p$ beam and the reverse $\bar{p}$ beam are transferred from the Main Ring to the Tevatron in straight section $E$ at $150 \mathrm{GeV}$. Each transfer beam line is a vertical dogleg spanning the 25.5-in. vertical separation between the two machines and is formed by two sections of Lambertson septum magnets. The beam is kicked horizontally into the Lambertson magnets in the Main Ring by fast kickers with both rise and fall times of about 0.5 usec. Similar kickers are placed downstream of the Lambertson magnets in the Tevatron to kick the beam horizontally onto the closed orbit. The horizontal positions and angles of the closed orbits in both the Main Ring and the Tevatron in the neighborhood of the septa are adjusted by bump magnets. A small quadrupole is placed at the crossing of the two transfer lines--one forward, one reverse--and is used by both beams to adjust for betatron matohing during the transfer.

6.2.5 Completion of Filling. After the first $\bar{p}$ bunch has been infected into the Tevatron, it remains there at $150 \mathrm{GeV}$ while the process is repeated the required number of times. Elther before or after the $\bar{p}$ injection, groups of proton bunches, properly spaced, are accelerated in the Main Ring and coalesced simultaneously at $150 \mathrm{GeV}$. The entire ensemble of proton bunches is then injected into the Tevatron. The entire counterrotating population is immediately accelerated to $1 \mathrm{TeV}$.

6.2.6 Tevatron RF Considerations. When the Tevatron is to be used for unilateral acceleration of protons, the location and spacing of rf cavities are not dictated by any consideration other than availble space. For simultaneous bilateral acceleration and subsequent storage of protons and antiprotons, however, some restrictions must be imposed upon cavity spacing. By appropriate spacing and phasing of the $r f$ fields in individual cavities, some aspects of bilateral operation can be optimized. The requirements for $\bar{p} p$ operation are:

(i) The rf system must create sufficient bucket area for simultaneous bilateral acceleration and storage of protons and antiprotons. Because the total longitudinal emittance of protons and antiprotons will almost certainly be different, the required bucket areas will not necessarily be the same. 
(ii) The rf system should provide the capability for moving the bunch collision point azimuthally over some reasonable range.

(iii) The system must allow for independent control of the phase and amplitude (bucket location and size) of the proton and antiproton buckets.

Elght Tevatron of accelerating cavities, each capable of sustaining $350 \mathrm{kV}$ accelerating voltage, will be required for the $\bar{p}$ program. Earlier Antiproton Source designs utilized four cavities, but the larger longitudinal emittance that stochastic cooling gives requires larger bucket area and therefore more cavities. These cavities are to be installed and operated in such a way as to make it possible to allocate four of the cavities to protons and four to antiprotons.

The cavities will be adequate for an acceleration rate of $34 \mathrm{GeV} / \mathrm{sec}$ at a synchronous phase of $30^{\circ}$. The acceleration time to $1000 \mathrm{GeV}$ will then be $25 \mathrm{sec}$. The bucket size is ample. The relative phase between the two sets of cavities is adjustable, so that the $\bar{p}$ and $p$ bunches can be rotated relative to each other to move the collision point to any desired azimuth.

After both beams are accelerated to full energy and phased properly, the low- $\beta$ quadrupoles discussed in Chapter 7 are slowly ramped to raise the luminosity to the design level.

Cavity spacing. It is necessary to consider cavity spacing in order to satisfy the requirements listed above for bilateral acceleration. We define a fundamental distance $\lambda$ in the Tevatron which is the orbit length of a synchronous particle divided by the $r f$ harmonic number

$$
\lambda=\frac{2000 \pi}{1113}=5.64527 \mathrm{~m} .
$$

A synchronous particle will travel this distance in one rf period.

Consider two accelerating cavities with their gaps separated by $(2 n+1) \lambda / 4$ meters ( $n$ is an even integer). If the accelerating voltage in the downstream cavity lags that of the upstream by $\pi / 2$ radians, then a proton moving downstream will see the voltages exactly in phase (modulo $2 \pi$ ). An antiproton moving upstream through the same pair of cavities will see the gap voltages exactly out of phase and if the voltages are equal it will not be affected by these two cavities. In a simllar way, the gap voltages of the two other cavities with the same separation can be adjusted so that the protons see no voltage and the antiprotons see the voltages added in phase. In Fig. 6-6a eight accelerating cavities are shown in a configuration that couples cavities 1,3,5,7 to (right moving) protons while cavities $2,4,6,8$ couple to antiprotons. The phase of the rf voltage exciting the even-numbered cavities is shifted by an arbitrary angle $\delta$. 
Failure Modes and the Collision Point. Figure $6-6 \mathrm{~b}$ shows a phasor diagram of the effect of the eight (equal) cavity voltages on a proton. If the angle $\delta$ is as shown and one of the even numbered cavities fails suddenly, the amplitude and phase of the proton voltage change. This will result in coherent dipole motion of the proton bunches about the new bucket center with subsequent serious dilution of the proton distribution and longitudinal oscillation of the collision point. (The same failure will result in a reduction of the antiproton bucket size, but no phase shift, so dilution will be less severe). If the angle $\delta$ is $\pm \pi / 2$, a failure of any cavity will result only in a minor change in both bucket heights but no phase shift or collision point motion. So while adjustment of the angle $\delta$ can be used to move the collision point during specified periods of detector calibration or adjustment, it appears sensible to set the 'normal' colliding point such that $\delta$ is $\pm \pi / 2$.

If $\delta=-\pi / 2$, collision can occur at points separated from the gap of cavity 8 by integral numbers of half waves. If $\delta=-\pi / 2$, the collisions are keyed (by half wave lengths) to the location of the gap of the cavity ${ }^{1}$.

Fixed-Target Operation. During fixed-target Tevatron operation, it is a simple matter to reconfigure the voltage fan-out system so that the entire voltage developed by the eight of cavities is applied to the protons.

\subsection{Tolerances and Beam Lifetime}

6.3.1 Tolerances and Beam Lifetime. As long as the frequencies of the $p$ and the $\bar{p}$ rf systems are locked and identical to each other, there is no problem with the accuracy in transverse aiming of the two beams. The horizontal displacement $\Delta x$ of a beam at the collision point is given in terms of the frequency error $\Delta f$ by

$$
\frac{\alpha_{p}}{\eta} \frac{\Delta f}{f},
$$

where $Y=1067$ at $1000 \mathrm{GeV}, \gamma_{t}=\gamma$ at transition $=18.75$, and $\alpha$ is the dispersion function at collision point $\cong 0.04 \mathrm{~m}$ with low- $\beta$. This gifes

$$
\Delta x=14 m \frac{\Delta f}{f}
$$

To ensure $|\Delta x|<0.01 \mathrm{~mm}$, say, we need a frequency accuracy of $|\Delta f / f|<0.7 \times 10^{-6}$ which, with the two systems locked in phase, would be difficult not to achieve. 
The longitudinal position of the collision point is determined by the relative phase of the $p$ and the $\bar{p}$ rf systems. To insure that the collision point lies within $\pm \Delta z$, we need a relative phase which stays within

$$
\pm \Delta \phi= \pm 4 \pi \frac{\Delta z}{\lambda}
$$

To get $\Delta z<1 \mathrm{~cm}$, we need $\Delta \phi<1.3^{\circ}$. When the two rf systems are phase locked, it is again not difficult to keep the relative phase accurate to within this IImit.

The stationary $\mathrm{rf}$ bucket area on the $1000-\mathrm{GeV}$ flat-top is $12.9 \mathrm{eV}$ sec which is ample to contain the beam bunch with longitudinal emittance of $2 \mathrm{eV}$ sec. The worst offender in attaining good lifetime for bunched beam is the noise in the $r f$ system. The rf noise blows up the longitudinal emittance and eventually causes the beam to leak out of the bucket. This effect was dramatically demonstrated by the experience in 1981 at CERN on the SPS collider. They had known for some time that the phase signal used in the feedback system was noisy. When the source of the noise was finally located and eliminated in October, the single-beam lifetime jumped from 20 hours to 200 hours. We should be able to obtain similar or even longer lifetimes at $1000 \mathrm{GeV}$. This is, of course, the lifetime of a single beam. The luminosity Iffetime is naturally shorter. A number of estimates of lifetime are discussed in Sec. 8.5.

This experience also indicates that during the $30-\mathrm{sec}$ storage time at $150 \mathrm{GeV}$ when the 6 beam bunches are sequentially injected, there should be no noticeable growth in the longltudinal emittance.

The average vacuum in the Tevatron is expected to be $10^{-10}$ Torr or better, a combination of cold and warm sections. 1 The growth rate of the transverse emittance due to gas scattering should also be negligible. A more complete discussion of gas scattering is given in Chapter 8 .

\subsection{Beam Abort}

In case of malfunction of components or to dispose of degraded beams, one needs an efficient beam-abort system to extract the beams quickly from the accelerator. The efficiency should be high enough so that heating by the small amount of stray beam is not enough to cause the superconducting magnets to quench.

The abort systems for both the $p$ and the $\bar{p}$ beams are located in straight section $C$. To avold quenching the superconducting magnet by stray beam, a horizontal kink in the orbit is produced by leaving out a half dipole each at $B 48-3$ and $C 11-2$ and adding conventional magnets near the middle of the straight section to make up for the 8 mrad bend. These 
conventional magnets have either iron or current septa as required to allow the exit of the aborted beam. These conventional orbit-kink magnets help to deflect and shield stray beam scattered off the septum during an abort, away from hitting the downstream superconducting magnets. To abort the forward-going $p$ beam, fast kickers at B48 kick the beam upward into a Lambertson magnet located on the upstream end of the straight section. The Lambertson magnet deflects the beam horizontally outward out of the Tevatron ring onto a graphite-aluminum dump target located outside the accelerator tunnel. For aborting the reverse $\bar{p}$ beam, the kickers are located at $\mathrm{C} 17$ and kick the beam upward directly onto a steel dump target situated on the downstream (upstream for the reverse $\bar{p}$ beam) end of the straight section above the unkicked circulating beam. All 6 beam bunches $\left(\Omega_{3} \times 10^{11}\right.$ each of $p$ and $\left.\vec{p}\right)$ are aborted at the same time. Both kickers are triggered simultaneously at a time when no beam bunch is boxed in between the two kickers. The reason the forward dump target is placed outside the accelerator tunnel is that it is used also to stop the much more intense proton beam of $3 \times 10^{13}$ proton/pulse and 2 pulses/min when the Tevatron is operated for fixed-target experiments.

After approximately 12 hours of colliding-beam operation, the luminosity has decreased appreciably, the degraded beam bunches are aborted and a new charge of beam bunches is injected.

\section{Reference}

1. Superconducting Accelerator Design Report, Fermilab, May, 1979 Sec. 5.2.6. 


\section{INTERACTION REGION}

As discussed in Chapter 6 , the $\bar{p}$ and $p$ bunches are placed at equal spacings around the clrcumference of the Tevatron, arranged so that one of their crossings occurs in the BO long straight section, the location of the Collider Detector Facility. There will also be crossings in the Do long straight section, where space is reserved for a second colliding-beam experimental area, at present in a primordial state of design.

The luminosity can be enhanced for given beam currents by focusing the beams down to narrow waists at the collision point, using extra quadrupoles on either side of the collision point. These quadrupoles give a decrease in the amplitude function $B$ and the low gives the narrow waist.

It is desirable for our purposes to achieve a minimum $B$ value of 1 meter. Given the Tevatron lattice and dimensions, it is possible to achieve this minimum either with a design that utilizes quadrupoles having gradients of $25 \mathrm{kG} / \mathrm{in}$. , but that requires replacement of a single normal-cell quadrupole on elther side of BO, at $A 48$ and B12, by longer quadrupoles, or with a design utilizing "superquadrupoles" capable of $50 \mathrm{kG} /$ in., but contained completely within BO (see Sec. 10.5). Either design uses four separately powered quadrupole buses and efther can be adiabatically varied from the normal $\beta^{*}=72 \mathrm{~m}$ configuration to $B^{*}=1 \mathrm{~m}$, while causing no betatron mismatch or manipulation outside the interaction region except for corection quadrupole changes to preserve the overall tunes and sextupole changes to maintain the desired chromaticity.

The optics for these two low- $\beta$ designs are described by D.E. Johnson in $\overline{\mathrm{p}}$ Note 169 . The Type-C design, which requires quadrupole gradients to reach $25 \mathrm{kG} / \mathrm{in}$., is shown in Table 7-I and Figs. 7-1 through 7-5. This design utilizes special long quadrupoles, $12 \mathrm{ft}$ and $13 \mathrm{ft}$, and allows for $15 \mathrm{in}$. of additional longitudinal space for the entrance and exit of cryogenic leads. (The $15 \mathrm{in.}$ is in addition to the $14 \mathrm{in}$. allowed for the cryostat plus magnet in standard Tevatron quadrupoles, whose overall length without a beam detector is $80 \mathrm{in.)}$. Type $C$ requires the replacement and separate powering of two normal lattice quadrupoles at the 48 and 12 locations. It also requires the $Q 4$ quadrupoles to be powered with a bipolar power supply in the adiabatic change from $\beta^{*}=72 \mathrm{~m}$ to $\beta^{*}=1 \mathrm{~m}$.

\subsection{Quadrupoles}

The quadrupoles discussed here are intended to use NbT1 superconducting wire equal to the best of the existing Fermilab wire, which has a short-sample limit of $5.5 \mathrm{kA}$ at $55 \mathrm{kG}$. The quadrupoles must be able to operate at $25 \mathrm{kG} / \mathrm{in.}$, a gradient previously achieved in a three-shell quadmupole at Fermilab. The effect of using very good wire is not yet known. Within the next few months, a standard Tevatron quadrupole will be built with the highest quality Fermilab wire to see if it can operate stably at such high gradient. NbTi wire with a smaller copper to 
superconductor ratio (1.2:1) and short-sample limit of $5.5 \mathrm{kA}$ at $6.5 \mathrm{~T}$ also exists at Fermilab and we expect to build a second test quadrupole with it for evaluation of its potential low- $\beta$ use.

The $Q$ quadrupoles at the 48 and 12 locations will have special additions ${ }^{1}$ to their cryostats to allow the main Tevatron superconducting magnet bus to bypass them. In addition, the $Q_{1}$ quadrupole at the 12 location, where longitudinal space is at a premium, includes the trim dipole, beam detector, quench stopper, and safety leads for the Tevatron lattice, which would normally be located in the adjacent spool piece if space permitted.

\subsection{Power Supplies and Bus}

Four separate power supplies and associated circuits will be needed for the $1-m B^{*}$ design. The supplies will be modifled Tevatron holding supplies capable of providing up to $5500 \mathrm{~A}$. The supplies will be located in an annex to the $B O$ service building and the power will be transported to the magnets via water-cooled copper bus (total length of 4 circuits is 2700 $f t)$. For a current of $5000 \mathrm{~A}$, the power consumed by the bus is $550 \mathrm{~kW}$. Power will enter the magnets through lead boxes constructed especially to fit the 15-in. space provided for them.

\subsection{Refrigeration}

Liquid He refrigeration needs are as follows: quadrupoles ( $50 \mathrm{~W}$ ), four pairs of $5 \mathrm{kA}$ leads at $10 \mathrm{~W}$ each $(40 \mathrm{~W}+56 \mathrm{l} / \mathrm{hr})$, two turnaround boxes at $10 \mathrm{~W}$ each $(20 \mathrm{~W})$, two feed boxes $(45 \mathrm{~W})$ and $\mathrm{U}$ tubes $(5 \mathrm{~W})$, for a total of $160 \mathrm{~W}$ plus 60 liters per hour. This refrigeration need can be met initially by the $A 4(C 4)$ and $B 1(D 1)$ satellite refrigeration systems. Later, it may be desirable to provide stand-alone refrigeration, and it would certainly be needed if operation at $1.8 \mathrm{~K}$ is desired. The 60 liters per hour of liquid He would come from the Central Helium Liquifier through the $A 4$ and $B 1$ refrigerator and magnet systems. The estimated $\mathrm{LN}_{2}$ requirement is $250 \mathrm{~W}$. Refrigeration estimates for the interaction-region detector magnets are not included here, because it is expected that those needs will be satisfied with but separate refrigeration.

\subsection{Vacuum}

Pressure in the interaction region straight section should not exceed $10^{-9}$ Torr. This means the warm vacuum pipe through the detector plus a transition piece on elther side will require special preparation (possible bakeout). The transition pieces will contain isolation valves and ion pumps, as well as the connections to turbomolecular and roughing pumps. 
Table 7-I. TYPE C LOW- $B$ QUADRUPOLE PARAMETERS (Values in $\mathrm{kG} / \mathrm{m}$ )

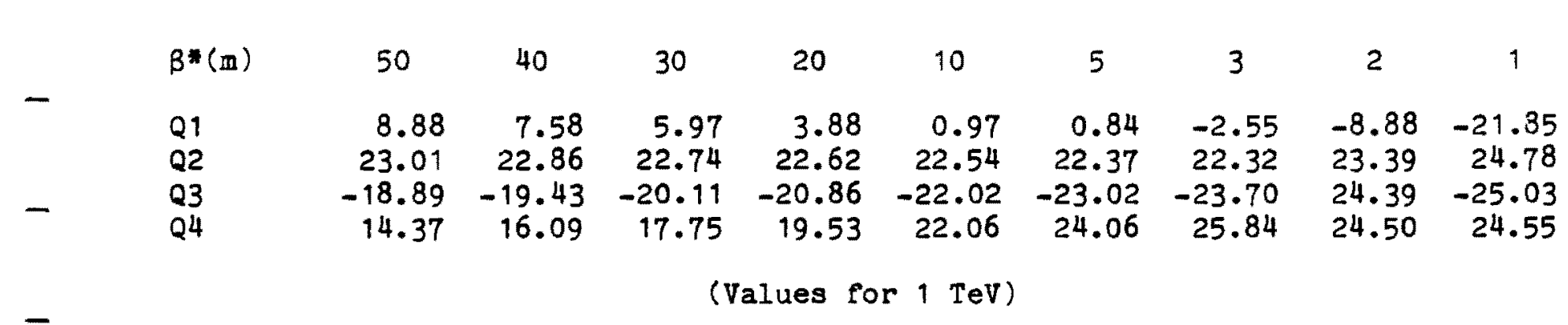




\section{PERFORMANCE AND LUMINOSITY}

In this section we describe the colliding-beam performance at $1000 \mathrm{GeV}$ in the Energy Saver. For this purpose, we assume that beams of protons and antiprotons have been accelerated and are circulating in opposite directions so they collide in the center of the low-beta insertion in BO, as well as at other azimuths.

\subsection{Beam Geometry}

As an example, it is possible to create three bunches of antiprotons each with $8 \times 10^{10}$ particles and each with a longitudinal phase area of $3 \mathrm{eV}-\mathrm{sec}$, which we define as the area including 95\% of the population with a biGaussian distribution. To prepare the necessary number of antiprotons in the Accumulator takes a little more than two hours.

Similarly we can assume (see Sec. 6.2.5) that three proton bunches, each with $10^{1}$ particles and a longitudinal area of $3 \mathrm{eV}$-sec coexist, with the antiproton beam. Both beams have equally spaced bunches since the harmonic number 1113 can be divided by 3. In principle there are, therefore, six collision regions equally spaced. We assume here that the two beams are not kept separated by special electrostatic deflectors, the design of which appears to be difficult because of lack of space in the Energy Saver and because of the large rigidity of the particles. An important parameter to determine the bunch dimensions is the peak $r f$ voltage $\mathrm{V}$ at $53.1 \mathrm{MHz}$. We assume here that two sets of four cavitles exist and that orthogonal control of the two beams is possible. In this mode of operation, there will be a total of $1.4 \mathrm{MV} / \mathrm{turn}$ for each beam.

Using the equations of Chapter 2, which apply to the Tevatron as well as to the Main Ring, we obtain for the rms bunch length $\sigma_{0}=40 \mathrm{~cm}$ and for the rms momentum spread $\sigma / p=1.2 \times 10^{-4}$. The area of the stationary bucket is $12.7 \mathrm{eV}-\mathrm{sec}$, fBur times larger than the bunch area. Finally the phase-oscillation period is $T_{s}=27 \mathrm{msec}$.

\subsection{Beam Cross Section at the Collision Point}

As a result of the transverse stochastic cooling in the Debuncher and Accumulator Rings, and because the two beams have roughly the same number of particles, the two beams have the same emittance, which is also the same in both horizontal and vertical planes. The normalized emittance is assumed to be $24 \pi \mathrm{mm}$-mrad. That is, $\varepsilon_{y}=\varepsilon_{\mathrm{H}}=0.023 \pi \mathrm{mm}$-mrad at 1000 GeV. This includes $95 \%$ of the beam with bigâssian distributions.

A low-beta figure of $1 \mathrm{~m}$ is expected in both planes and therefore 


$$
\sigma=0.062 \mathrm{~mm}
$$

or

$$
\sigma^{2}=4 \times 10^{-5} \mathrm{~cm}^{2}
$$

Since the low-beta insertion has very small dispersion, there will be negligible contribution to the beam size from momentum spread.

\subsection{Luminosity}

Because the low-beta value of $1 \mathrm{~m}$ is larger than the rms bunch length $(0.4 \mathrm{~m})$, a formula for luminosity valid for constant beam sizes is a good approximation. Thus

$$
L=\frac{N_{p} N_{\bar{p}} B f_{0}}{4 \pi \sigma^{2}},
$$

where $N_{D}=10^{11}$ is the number of protons per bunch, $N_{\vec{D}}=8 \times 10^{10}$, the number of antiprotons per bunch, $B=3$, the number of bunches per beam, and $f_{0}=4.77 \times 10^{4} \mathrm{~Hz}$, and the revolution frequency. This gives

$$
L=2.2 \times 10^{30} \mathrm{~cm}^{-2} \mathrm{sec}^{-1} .
$$

This luminosity figure is definitely within reach with the methods and the techniques described in this report. Several alternative luminosity scenarios can be invented. For instance, it is possible to replenish the collider every hour with one single bunch of protons and antiprotons with $10^{11}$ particles each, for a luminosity of $10^{30} \mathrm{~cm}^{-2} \mathrm{sec}^{-1}$.

Higher luminosity figures can also be expected, as high as $6 \times 10^{30}$ $\mathrm{cm}^{-2} \mathrm{sec}^{-1}$, by improving either the stochastic cooling (a factor of two), or the beam intensity in the Main Ring and Tevatron (also possibly by a factor of two) or with 6 bunches per beam instead of 3 as assumed here.

\subsection{Beam-Beam Tune Shift}

Collisions are head on, the emittances of both beams in both planes are all equal, and the lattice functions are approximately the same in both planes. Therefore, the beam-beam tune shift is the same for both beams (except for possible variation of charge from bunch to bunch), is independent of the beam energy, and does not depend on the lattice functions. The Iinear beam-beam tune shift is given by 


$$
\xi=\frac{3 \mathrm{Nr}_{0}}{2 \varepsilon_{\mathrm{N}}},
$$

where $N=10^{11}$ is the number of particles per bunch, $\varepsilon_{N}=24 \pi$ mm-mrad is the normalized emittance and $r_{0}=1.535 \times 10^{-18} \mathrm{~m}$ is the classical proton radius. We have

$$
\xi=0.003 / \text { crossing }
$$

Even with a low-beta insertion, the beam cross-section in the other five collision regions with normal $\beta$ values is round and the contribution to the beam size from the momentum spread is negligible. Therefore the tune shift per crossing, in case the two beams are not separated, is the same at each crossing as long as the collision is head-on or at least at an angle $\theta \ll \sigma / \sigma_{e}$. If there are a total of 6 crossings, the total tune-shift per turn

$$
\xi_{\text {tot }}=6 \xi=6 \times 0.003=0.018
$$

\subsection{Single-Beam and Luminosity Lifetime}

We have investigated three possible sources of Iffetime deterioration in the Tevatron: residual gas, intrabeam scattering and beam-beam effects. At the same time an analysis of perlodic or random crossings of nonlinear resonances has been initiated. This will help to determine not only possible limits on the amount of nonlinearities in the Energy Saver superconducting magnets, but also on the amount of coherent and incoherent nolse amplitude allowable.

8.5.1 Effects of Residual Gas ${ }^{2}$. The following effects have been Invest1gated:

(1) Multiple Coulomb Scattering

(ii) Single Coulomb Scattering

\section{(iii) Nuclear Scattering}

The first effect causes a constant beam-emittance growth, which leads to both a luminosity decay as well as a single-beam lifetime if an aperture limitation is taken into account. Nevertheless the single-beam decay due to multiple Coulomb scattering is quite negligible. The second and third effects cause an intensity decay, but the last effect is more important than the second one. 
The average vacuum pressure expected in the Tevatron is $10^{-8}$ Torr in the warm regions and $5 \times 10^{-11}$ Torr in the cold regions with the following gas composition:

$$
\begin{aligned}
& \text { warm region: } 60 \% \mathrm{H}_{2} \text { and } 40 \% \mathrm{CO} \\
& \text { cold region: } 75 \% \mathrm{H}_{2} \text { and } 25 \% \mathrm{He}
\end{aligned}
$$

The intensity decay per beam due to single Coulomb and nuclear scattering is

$$
\frac{1}{I} \frac{d I}{d t}=-1.07 \times 10^{-6} / \mathrm{sec} .
$$

The emittance growth due to multiple Coulomb scattering is

$$
\frac{1}{\varepsilon} \frac{d \varepsilon}{d t}=1.3 \times 10^{-6} / \mathrm{sec}
$$

These effects combined lead to a luminosity decay of

$$
\frac{1}{\mathrm{~L}} \frac{\mathrm{dL}}{\mathrm{dt}}=-3.44 \times 10^{-6} / \mathrm{sec},
$$

a loss of $13 \%$ of the luminosity in 10 hours.

8.5.2 Intrabeam Scattering ${ }^{2}$. To estimate intrabeam scattering diffusion rates for the colliding-beams mode, we have used the same computer code we used to estimate intrabeam scattering for the Accumulator (see Sec. 5.1.4). For our estimate, we have used the actual Tevatron lattice with the low-beta insertion. From the computer code we have empirically found the following formulas for diffusion rates, which apply to a bunohed beam at $1000 \mathrm{GeV}$. For the momentum spread

$$
\frac{1}{\tau_{p}}=\frac{1}{\sigma_{p} / p} \frac{d \sigma_{p} / p}{d t}=\frac{{ }_{p} I_{p}}{\left(\sigma_{p} / p\right)^{m_{p}} \varepsilon^{r_{p}}}
$$

and for the horizontal betatron emittance 


$$
\frac{1}{\tau_{x}}=\frac{1}{2 \varepsilon_{x}} \frac{d \varepsilon_{x}}{d t}=\frac{A_{x} I_{p}}{\left(\sigma_{p} / p\right)^{m_{x} \varepsilon^{r}}}
$$

There is actually damping for the vertical betatron emittance.

In these equations $\sigma_{p} / p$ is the rms momentum spread, $\varepsilon=2 \sigma^{2} / \beta$ the rms betatron emittance, whikh we assume to be the same in both planes, and $I_{p}$ is a measure of the bunch peak current

$$
I_{p}=\frac{N_{B} e B c}{2 \sqrt{ } \pi \sigma_{e}} \text {, }
$$

where $\mathrm{N}_{\mathrm{B}}$ is the number of particles per bunch, $\beta_{c}$ the particle velocity, and $\sigma_{e}$ is the rms bunch length. Also

$$
\begin{aligned}
& A_{p}=5.7 \times 10^{-23} \mathrm{Amp}^{-1} \times \mathrm{m}^{r_{p}} \times \text { hour }^{-1} \\
& A_{x}=12.6 \times 10^{-23} \mathrm{Amp}^{-1} \times \mathrm{m}^{r_{x}} \times \text { hour }^{-1} .
\end{aligned}
$$

If $\sigma_{p} / p=1.2 \times 10^{-4}, \sigma_{e}=40 \mathrm{~cm} \mathrm{~N} B=10^{11}$, then $I_{p}=3.4 \mathrm{~A}$, and if $\varepsilon=$ $0.0079 \mathrm{~mm}-\mathrm{mrad}$, then

$$
\begin{array}{ll}
m_{p}=2.2 & r_{p}=1.4 \\
m_{x}=0.7 & r_{x}=2.0
\end{array}
$$

We obtain as initial diffusion rates

$$
\begin{aligned}
& \frac{1}{\tau_{p}}=5.2 \times 10^{-6} / \mathrm{sec} \\
& \frac{1}{\tau_{x}}=1.1 \times 10^{-6} / \mathrm{sec} .
\end{aligned}
$$

The actual variation of $\sigma_{p} / p$ and $\varepsilon_{x}$ is obtained by simultaneous integration of Eqs. (8-1) and (8-2). The result is shown in Fig. 8-1.

There is a loss of luminosity caused by intrabeam scattering diffusion in the horizontal betatron oscillation. Assuming vertical and horizontal coupling, the loss of luminosity after 10 hours is about $7 \%$. After the same period of time ( 10 hours), the bunch area has increased by $32 \%$. 
8.5.3 Beam - Beam Effects. Beam-beam effects have been extensively simulated on the computer. Several issues have been discussed, studied, and, we hope, resolved.

A systematic search for Arnol'd diffusion for the Tevatron parameters has given negative results. ${ }^{3,4}$ We have been able to simulate in some cases up to 20 minutes real time of collisions. From our data, we can extrapolate beam-beam lifetimes of several days. We believe the stability of the system comes from the "roundness" of the beam geometry and of the lattice functions. 5

We found that the addition of the nonlinear beam-beam interaction to a system already affected by external random nolse (such as gas scattering) causes an enhancement of the diffusion rates. ${ }^{6}$ The largest enhancement encountered was a factor 6 for a beam-beam tune shift of 0.06 and in proximity to the fourth-order resonance.

We have investigated beam-beam interactions with the beam centers offset or oscillating around each other. ${ }^{7}$ This could be caused by either a dipole oscillation or a finite dispersion in the collision region coupled to the momentum oscillation. We have not noticed any effect of significance.

Of more serlous concern are the effects created by betatron tune oscillations. If proper care is not taken, it is possible to cause an emittance growth of a factor two in a few minutes. ${ }^{B}$ Fortunately, we have found a threshold and the growth can be tuned out by elther adjusting the betatron tunes or by improving the power-supply regulation to better than $10^{-4}$ and flattening the lattice chromaticity.

At present, we are investigating multiple crossings per revolution, two or six as opposed to one.

8.6 Beam-Stability Considerations in the Tevatron. As we have seen, each beam is made of three bunches, each with $10^{11}$ particles and a longitudinal area of $3 \mathrm{ev}-s e c$. The beam intensities, both average and peak, are very modest and dilute. A considerable effort has been made in the recent past to estimate the longitudinal stability of each beam for both individual bunch and bunch-to-bunch modes. $9,10,11$ The beam wall impedance expected in the Tevatron was estimated to be $\left|z_{n}\right| / n<1$ ohm. Because of the low

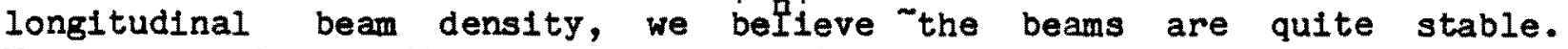
Moreover, a longltudinal damper operating on each individual bunch is planned.

Fewer calculations have been done for the case of transverse instabilities. But again, we do not foresee major problems. In addition, a transverse active damper has been proposed ${ }^{12}$ that will be fast enough to operate on individual bunches in both radial and vertical mode of oscillations. 
1. H. Mizuno, S. Ohnuma, A. G. Ruggiero, "Vacuum and Beam Lifetime in the Doubler," UPC No. 119, Fermilab 21, 1979.

2. A. G. Ruggiero, "Revised Intrabeam Scattering Calculations," Fermilab $\bar{p}$-note 192 (1982), unpublished.

3. D. Neuffer, A. Riddiford, A. Ruggiero, "A Search of Arnol'd Diffusion in the Beam-Beam Interation," FN 333, Fermilab, Apr. 1981.

4. D. Neuffer, A. Riddiford, A. Ruggiero, "Long Time Simulation of the Beam-Beam Interaction," FN-343, Fermilab, July 1981.

5. A. G. Ruggiero, "Particle Accelerators" (to be published in Vol. 12, No. 1).

6. D. Neuffer, A. Riddiford, A. Ruggiero, "Diffusion Enhancement by the Beam-Beam Interaction in 1-D Simulations," FN-357, Fermilab, Oct. 1981 .

7. D. Neuffer, A. Riddiford, A. Ruggiero, "Effects of Transient and Modulation of Beam Displacement on Long-time Stability with the Beam-Beam Interaction," FN-358, Fermilab, March 1982.

8. D. Neuffer, A. Riddiford, A. Ruggiero, "Simulations of the Beam-Beam Interaction with Time Modulation at the Tevatron p尹̄-Collider," FN-359, Fermilab, Feb. 1982.

9. A. G. Ruggiero, "Individual Bunch Longitudinal Instabilities," Fermilab, UPC-72, Jan. 1979.

10. A. G. Ruggiero, "Bunch-to-Bunch Longitudinal Instabilities," Fermilab, UPC-81, Jan. 1979.

11. King-Yuen Ng, "An Estimate of the Longitudinal Coupling Impedance of the Energy Doubler," Fermilab, UPC-150, May 1981.

12. W. Miller, "Transverse Active Dampers for the Tevatron," Fermilab, UPC-36, Jan. 1979. 


\section{COMMISSIONING AND OPERATION; DIAGNOSTICS AND CONTROLS}

\subsection{Commissioning}

Because of the tight construction schedule and the desire to have $p \bar{p}$ collisions as soon as possible, we must minimize the commissioning time for the $\bar{p}$ source. In particular, we propose a rather complete set of diagnostic devices as well as emphasis on the ability to inject protons parasitically into the two $\bar{p}$ rings from the Booster either via the Main Ring or a direct transfer line from the Booster to the Debuncher.

By reversing the magnetic-field polarities in the Debuncher, Accumulator and appropriate transfer lines, all the functional devices can be debugged and optimized with protons from the Booster. (It is necessary to have the test protons circulate in the same direction as the $\bar{p}$ 's in order to allow the testing of the stochastic-cooling systems). The tune diagrams of the two rings can be explored and the acceptances optimized before $\bar{p}$ 's must be produced by the Main Ring. In addition, the high proton density from the Booster will allow the simulation of dense $\bar{p}$ stacks in the Accumulator so that instabilities and their cures can be investigated. This work is particularly important because the ultimate luminosity of the Tevatron $\bar{p} p$ collider may well be determined by the maximum core density in the Accumulator.

For first operation, however, the emphasis will surely be on rapid $\bar{p}$ production rather than maximum core density. This means that measurement of ring acceptances and transfer efficiencles will have high priority during initial running-in. The stochastic-cooling systems will also require some development to achieve their anticipated flux acceptance. The two rf systems in each ring can also be debugged with protons from the Booster. The $\bar{p}$ production process can be studied and optimized independent of much of the activity in the Debuncher and Accumulator. Activities in the Main Ring tunnel and possible interferences with the fixed-target high-energy physics program may make studying the $\bar{p}$ production more difficult, although parasitic operation should be possible.

For transier of antiprotons into the Main Ring and Tevatron, occasional dedicated study perlods will be useful and necessary before collider operation.

\subsection{Diagnostics}

9.2.1 Accumulator and Debuncher Diagnostics.

1. Schottky signals. From the Schottky signals one can measure the betatron tunes and momentum distributions of the beams. For low-intensity $\bar{p}$ beams of $10^{7}$ particles, as in the Debuncher, one may have to develop resonant Schottky plckups to enhance the sensitivity. One also uses the Schottky signals to determine the effectiveness of the stochastic cooling. 
2. Position electrodes. Information from the horizontal or vertical position pickups placed near the quadrupoles in each ring is necessary to correct closed-orbit errors, measure position vs momentum dependence and correct injection errors. These electrodes will also be used for ion clearing if that is found to be necessary. The newly developed plckups and electronics used for the Tevatron seem to have the desired sensitivities.

3. Beam transformers. A sensitivity of $1 \mu \mathrm{A}$ or approximately $10^{7} \overline{\mathrm{p}}$ will be necessary. Some development work may be necessary to achieve this level.

4. Profile monitors. To see the effectiveness of the betatron stochastic cooling and to see resonance blow-ups, beam profiles must be measured. For the high-intensity core, a flying-wire scanner as used at the CERN SPS would be ideal. A 50-im wire travelling at $10 \mathrm{~m} / \mathrm{sec}$ would only cause about $0.03 \%$ beam loss and $0.03 \pi \mathrm{mm}-\mathrm{mr}$ emittance growth per scan. The ionization monitor as developed for the Fermilab cooling ring would also be ideal, especially for the low-intensity beams of the Debuncher. Horizontal and vertical scrapers at regions of high and low $\alpha_{p}$ are also planned.

5. Aperture scans. To scan the apertures and measure lattice parameters, the normal rf systems will be used to deposit beams at different momenta. Computer-controlled magnetic correction elements then can be varied to compare calculated vs, measured betatron tune variations. To study effects of large betatron amplitudes, the beam can be blown up with single-turn kickers or the 1 to $50 \mathrm{MHz}$ dampling system can be fed with reversed feed-back sign or noise.

\subsubsection{Targeting and Beam Transfer Diagnostics.}

1. The target area will be equipped with secondary emission monitors (SEM) and transformers in approprlate places. Because of the sensitivity of the lithium lens to an incident proton beam off-axis, the steering of the incldent beam will have to be closely monitored. Segmented Wire Ion Chambers (SWICs) will be used for automatic monitoring of the proton beam incident on the target.

2. All beam transfer lines w11l be equipped with SWICs or similar profile monitors, including at least 3 in each ring to allow matching of phase-space parameters. Some development may be needed to make a profile monitor suitable for injection matching and compatible with the accelerator vacuum.

3. Low intensity $\bar{p}$ beam Iines, $i . e .$, from the target to the Debuncher and between Debuncher and Accumulator, will have some 
high-sensitivity (low-mass) profile monitors such as proportional chambers.

\subsection{Controls}

The operation of the $\bar{p}$ source will eventually be controlled from the main control room (MCR) of the accelerator complex by the normal operation crew. Thus, the accelerator control network (ACNET) now being implemented for the Tevatron, which will eventually be used for the Booster, Linac, Main Ring and Switchyard, will be used for the $\bar{p}$ source.

Details of methods of interfacing devices in the $\bar{p}$ source to ACNET, e.g., via serial CAMAC, can and should be determined based on experience with the new Tevatron system. In any case, the control system for the $\bar{p}$ source will be compatible with and as similar as possible to the other accelerator systems controlled from the MCR. We expect that many of the procedures and programs developed during $\bar{p}$ running-in will become the backbone of the operational system.

For the commissioning period, at least one ACNET console will be needed in the gallery over the Debuncher and Accumulator. 
10. OPTIONS FOR FUTURE IMPROVEMENTS

The Antiproton Source design must include not only the best system for antiproton production utilizing presently known techniques, but also provisions for improvements either from new inventions or from operational experience. In this chapter, we discuss a number of possible improvements that could increase the peak luminosity or decrease the accumulation time, thus increasing the average Iuminosity. It is to be emphasized that these improvements are not part of the construction project.

\subsection{Momentum Cooling in the Debuncher}

As was pointed out in Chapter 4, there is time during the 2-sec Debuncher cycle for cooling after the initial debunching. It is planned to do transverse cooling as part of the inttial design. But it is also possible to cool in momentum by a factor 2 during the same time. This will reduce the cooling power needed in the Accumulator or increase the initial cooling speed for the same power.

The momentum cooling will utilize a notch filter (Thorndahl method). The gain shaping is obtained with a long shorted cable that resonates at harmonics of the revolution frequency. This method is preferred to the Palmer method, where the gain variation is obtained with two palrs of pickups in difference mode in a large dispersion region. Because of the large electronic gain needed to obtain the desired cooling rate, thermal noise is a potential problem. Cooling would actually be impossible for our case with the Palmer method, since in it noise is not effectively filtered and would cause the equilibrium beam width to be larger than the initial beam spread. With the filter method, the thermal noise still predominates, but its effect is greatly reduced. The method is very similar to the one used for the precooling step in the CERN AA Ring.

At the start, the beam has a very small energy spread, between 25 and $30 \mathrm{MeV}$. To get a significant signal, large bandwidth and a large value of $\eta$ are required. We assume a bandwidth from $2 \mathrm{GHz}$ to $4 \mathrm{GHz}$ and $\eta=-0.004$. An even larger value of $n$ would be desirable, but then extremely high $r f$ voltages would be needed for debunching.

To sharpen the depth of the notches and to cut down thermal noise in the frequency region where there is no beam, we will use as the filter a cable whose delay matches the revolution period and whose far end is shorted. This filter creates notches not only at harmonics of the revolution frequency, but also at frequencies halfway between.

The overlap factor, the ratio of the beam Schottky band width to the separation of neighboring notches, ranges from 0.1 at the lower end to 0.2 at the upper end of the bandwldth. 
A lossless cable can have notches at desired frequencies by careful adjustment of cable length. Losses due to skin depth of the conductor shift the notches and make cooling ineffective. One possible method we are investigating is compensation of losses by adding a lossy cable of short length in parallel. The compensation is adjusted in either length or loss until the notches are restored to the proper frequencles. If $\alpha$ is the attentuation parameter and $l$ the length of the cable, for our calculations we have $\alpha \ell=0.3$ at $4 \mathrm{GHz}$. Thls corresponds to a 2-in. outer diameter vacuum-core copper cable at liquid-helium temperature. The assumed characteristic impedance of all the cables involved, as well as of the entire network, is 50 ohms.

The signal loss caused by beam dispersion and the cable delay between pickups and kickers is not a problem. If the distance between pickups and kickers is 0.35 times the circumference length, the fraction of signal lost is only $0.5 \%$ at the lower end of the bandwidth and $2 \%$ at the upper end.

The signal cable is approximately $125 \mathrm{~m}$ long. There is a time difference of about 150 nsec between the beam path length and the cable delay. Power amplifiers have electronie delays around 60 nsec.

The room-temperature performance of a copper cable seems to be adequate for the signal cable, where with a 4.45-in. outer diameter, we expect fractional losses between $18 \%$ at $2 \mathrm{GHz}$ to $25 \%$ at $4 \mathrm{GHz}$. These losses can be compensated by raising the electronic gain. The phase variation is also reasonably small; from $3 \%$ at $2 \mathrm{GHz}$ to $4 \%$ at $4 \mathrm{GHz}$.

To make cooling possible, differentiation of the signal in the network is required. This is accomplished with a short cable, one or two inches long, shorted at the far end.

Pickups and kickers will be made of strip-lines. They will be used for both momentum and betatron cooling. Pickups and kickers have the same configuration. They are grouped in four tanks. Each tank is $4.5 \mathrm{~m}$ long and accommodates $4 \times 128$ plates. The plates are shorted to ground at the upstream ends and their downstream ends are combined together. The combination is done under vacuum, and there are only two leads coming out at the downstream end of each tank.

\subsection{Target Development}

In order to achleve targets that would survive smaller beam spots (larger energy density deposited) the following approaches to target design are under study:

(1) Laminated targets with the plane of the laminations perpendicular to the proton beam would decrease the amount of material flow within the plastic zone. 
(ii) Filamented targets would allow the introduction of slip planes across large temperature gradients, resulting in a lowering of the stresses.

(iii) Powder targets produced by embedding high-density particles in graphite by powder metallurgy would incorporate the excellent shock and high-temperature properties of graphite, allowing enough energy deposition to melt the metal particles. The lower antiproton yield resulting from the lower average target density could be overcome by a much smaller beam spot.

A research and development program to test these ideas will be underway before the $\bar{\delta}$ source becomes operational.

\subsection{Improvements in Stochastic Cooling}

The pace of electronic development is strikingly rapid and we expect that high-power, large-bandwidth systems will be significantly improved in the next decade. In particular, we expect that bandwidth will be increased by raising the high-frequency end of amplifier systems and that electronic noise will be lowered. Either of these developments could aid the cooling in the Accumulator. If we could predict what these advances might be, we could design them into the system. Failing that, we can make the design as flexible as possible, leaving aperture and straight-section space for testing and installation of these systems. We plan to utilize the shorter 4-ft and 10-ft straight sections for development tests of new cooling systems.

\subsection{Electron Cooling of the Core}

It would be possible at some later time to add an electron core-cooling system to the Accumulator. This system could be used in several different ways:

(1) to counteract intrabeam scattering of a stochastically cooled antiproton beam and to increase its density beyond what stochastic cooling can achieve;

(ii) to use the electron beam directly as the accumulating cooling system. This would require that the transverse emittance of the antiproton beam be reduced stochastically to approximately $0.5 \pi$ mm-mrad before electron cooling. This stochastic cooling could be done with large momentum spread to get good mixing. Use of electron cooling would then give very high accumulation rates.

We can expect to do this core cooling at full energy, $8 \mathrm{GeV}$. Electron beams based on electrostatic generators are now being developed for 
free-electron laser studies with electron energies of 3 to $6 \mathrm{MeV}$ and beam currents of 5 to 10A. The cooling region would be unmagnetized and could be installed in one long straight section of the Accumulator.

For this application, an electron beam of good emittance, good reliability, and very good collection efficiency would be required. We are now engaged in a program in collaboration with National Electrostatics Corporation to measure emittance and collection efficiency and to Investigate long-pulse operation.

\subsection{The Overpass - A Main Ring Bypass}

It is of interest to consider bypassing the Main Ring around the Collider Detector at BO. There are at least two advantages of bypassing the detector:

(i) It allows for the elimination of the asymmetric Main Ring hole through the Detector.

(ii) It may allow for accumulation of more $\bar{p}$ 's while collider experiments are going on. This increases the average luminosity of the entire system by eliminating the pause of several hours while $\bar{p}$ 's are being accumulated.

To make a bypass, space must be created for additional bending elements. We propose to carry this out by limiting Main Ring operation to energies less than $200 \mathrm{GeV}$. Sets of 4 magnets around the bypass are ramped at twice the field. The outer pair provides the normal horizontal bend with the same bend center as the original four magnets, while the inner pair is rolled to provide vertical bending (with automatic tracking).

It is important in the sequence to match the dispersion functions outside the bypass and to minimize any new dispersion introduced outside the bypass. The solution chosen introduces less than $1 / 4 \mathrm{~m}$ of vertical dispersion, which is negligible. It allows $5 \mathrm{ft}$ of shielding above the bypassed beam in the Collision Hall.

The cost of this bypass has been estimated as slightly less than $\$ 6 \mathrm{M}$. Provision for its inclusion in the future has been made in the design of the Collision Hall.

\subsection{Low-beta Improvements}

It is of considerable interest to pursue the development of quadrupoles with gradients in excess of $25 \mathrm{kG} / \mathrm{in}$. A gradient of 36.5 $k G / 1 n$., for example, would permit the Type-C low-B design to achieve $a$ minimum value of $B^{*}=0.5 \mathrm{~m}$, and such a gradient would allow all of the 
quadrupoles to be shorter, thereby allowing more room for connections, etc. Such a solution would have a rather large maximum beta value that might present some problems. It may be desirable not to disturb the normal Tevatron with the quadrupole replacement at the 48 and 12 locations, as is done in the Type-C design of Chapter 7. If one could produce quadrupole gradients of $50 \mathrm{kG} / \mathrm{in} ., \quad a$ low- $\beta$ design entirely contained within the straight section could be used, with somewhat smaller maximum beta values. This is shown in Table 10-I and Figs. 10-1 and 10-2. A gradient of some $68 \mathrm{~kg} / \mathrm{in}$. will allow a $\beta^{*}$ of $0.5 \mathrm{~m}$ without too large a value of $\beta_{\max }$.

Table 10-I. TYPE D LOW-B QUADRUPOLE PARAMETERS

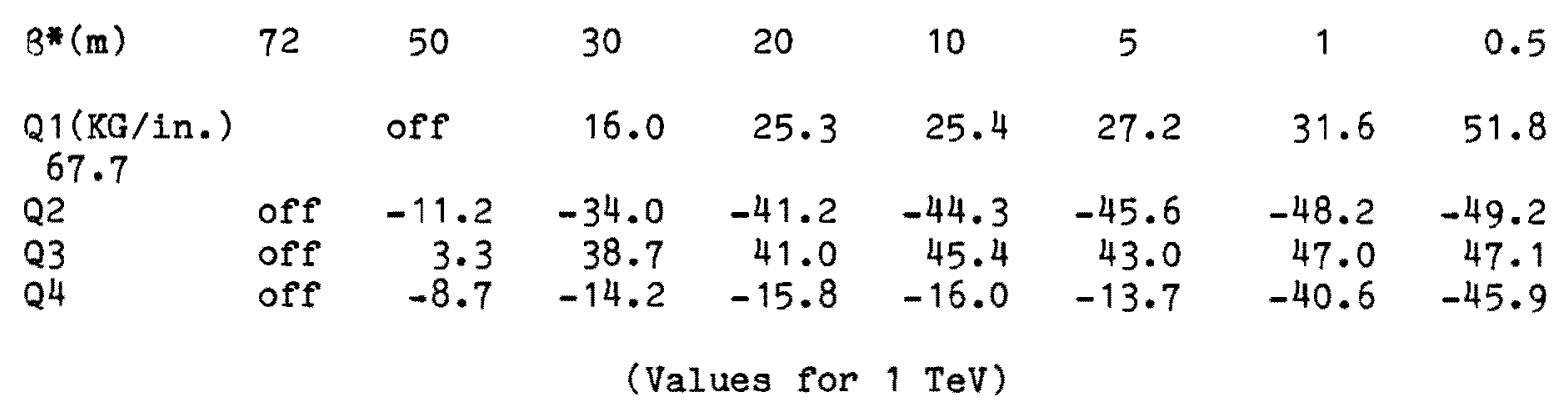

High-gradient quadrupole development would include investigation of $1.8 \mathrm{~K}$ operation, superconducting wire other than NbTi, many-shell quadrupole design, and other innovations. 


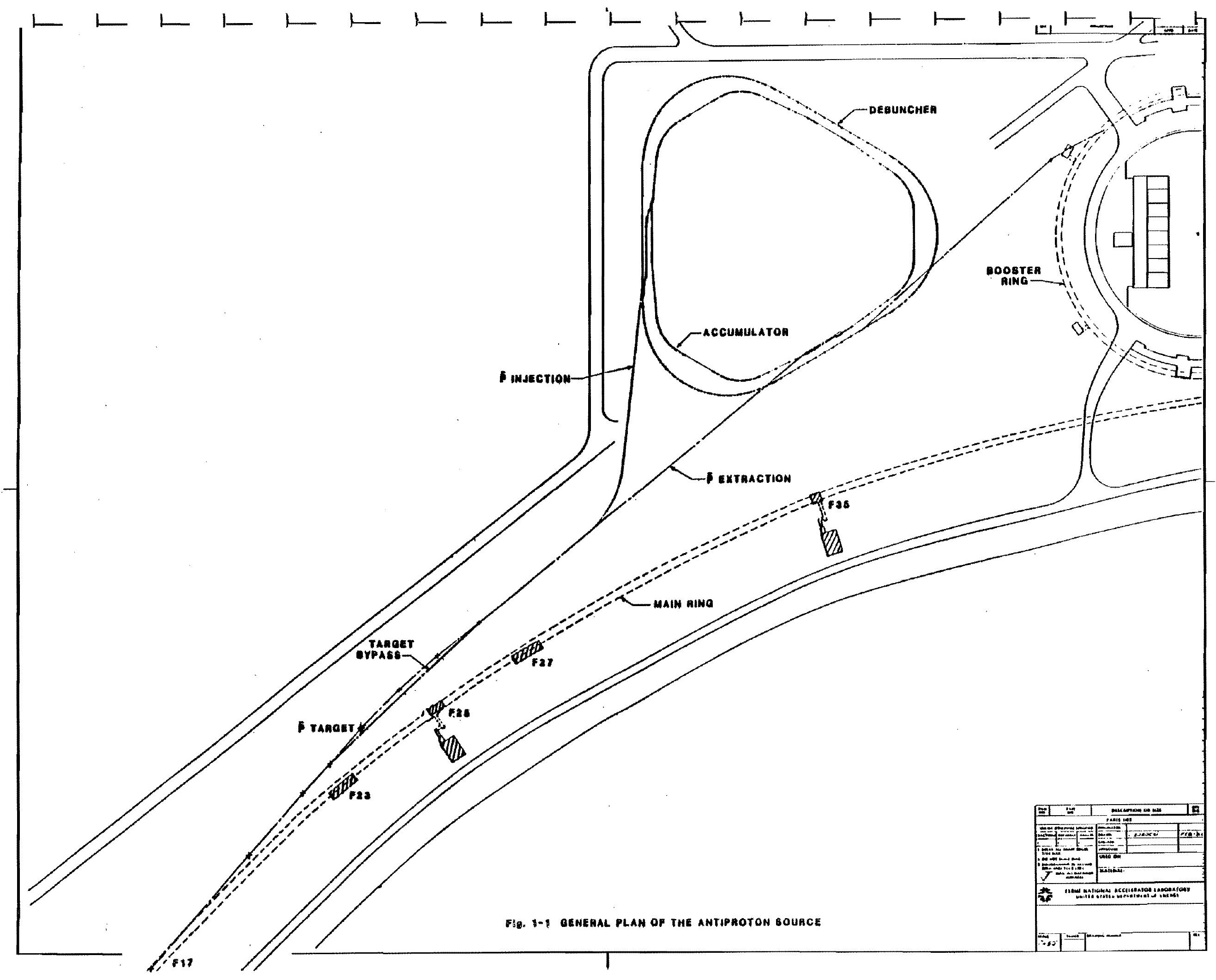




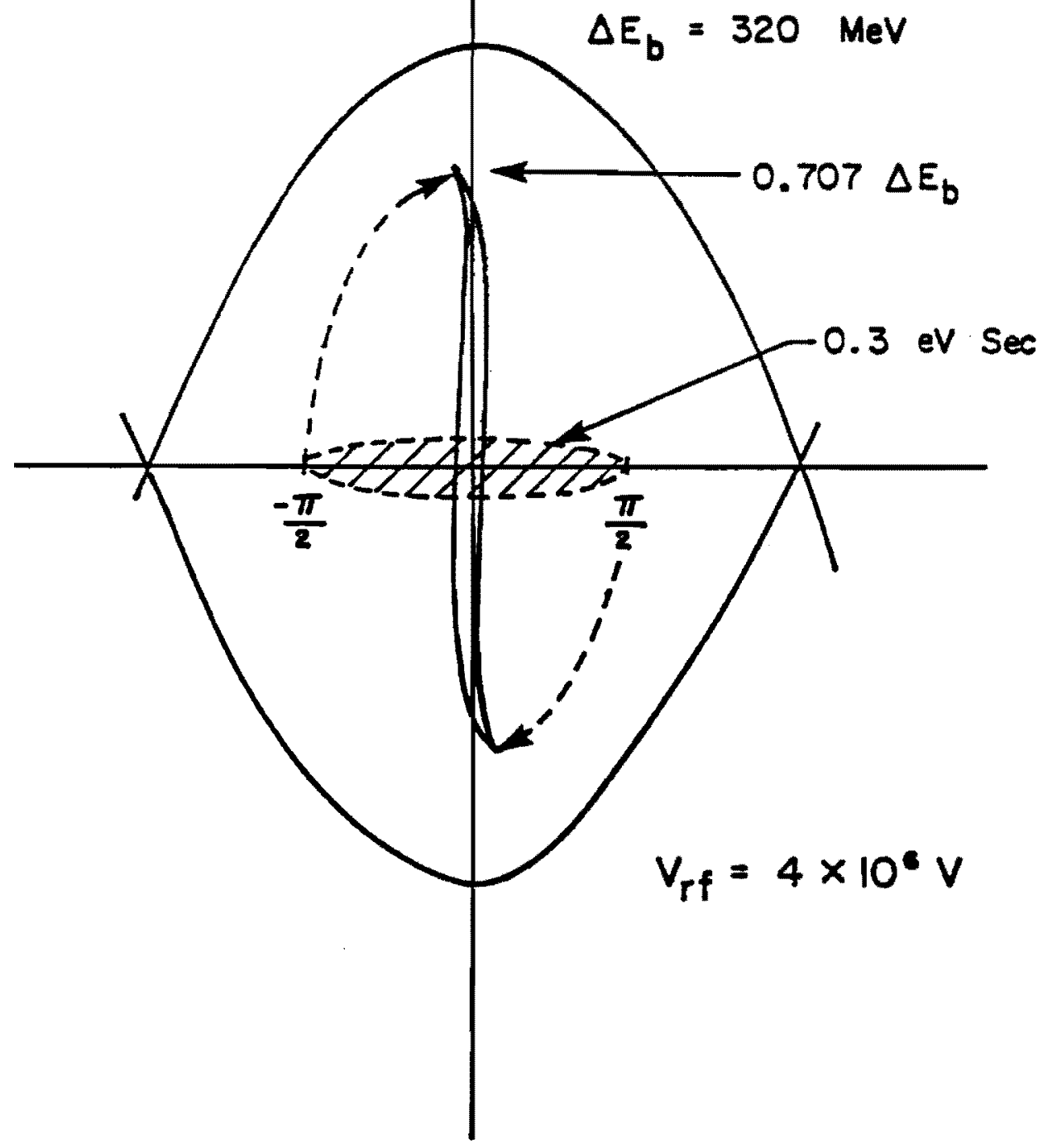

Fig. 2-I 

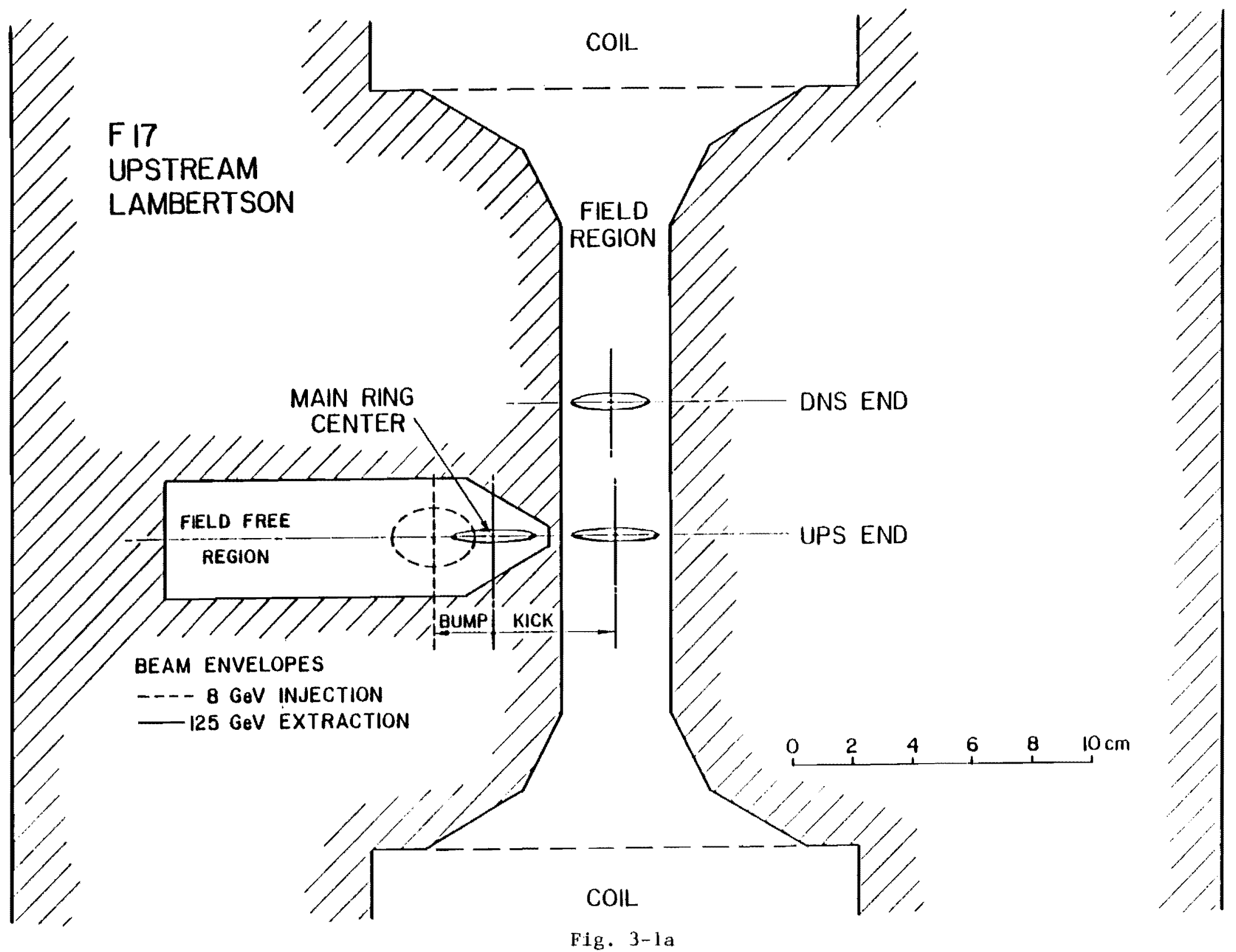

Fig. 3-1a 


\section{F 17}

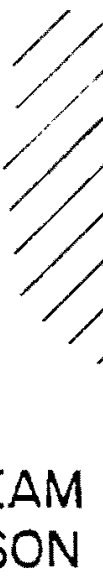

BEAM ENVELOPES

- - - $8 \mathrm{GeV}$ INJECTION

- $125 \mathrm{GeV}$ EXTRACTION DOWNSTREAM LAMBERTSON

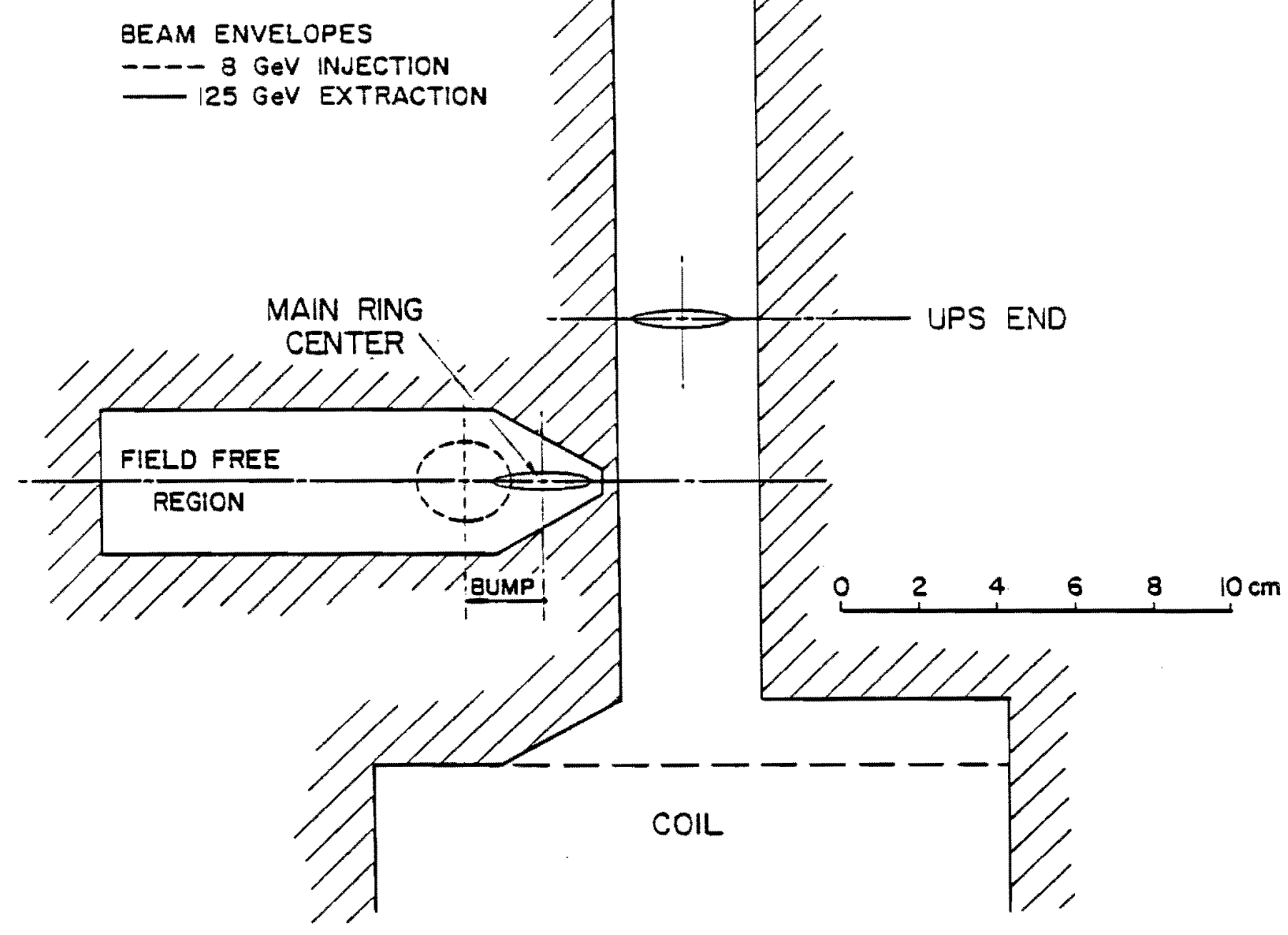

Fig. 3-1b 

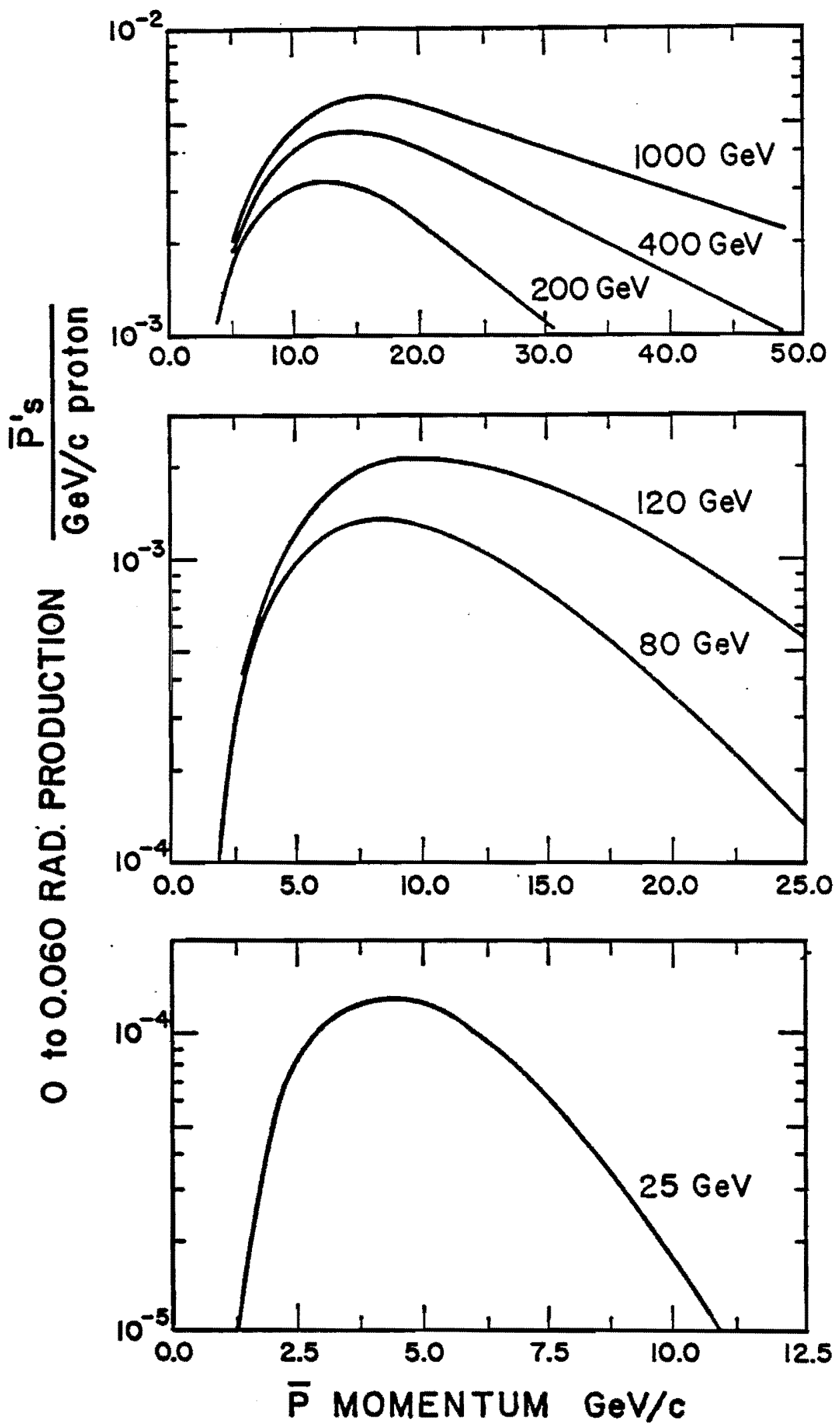

Fig. 3-3 


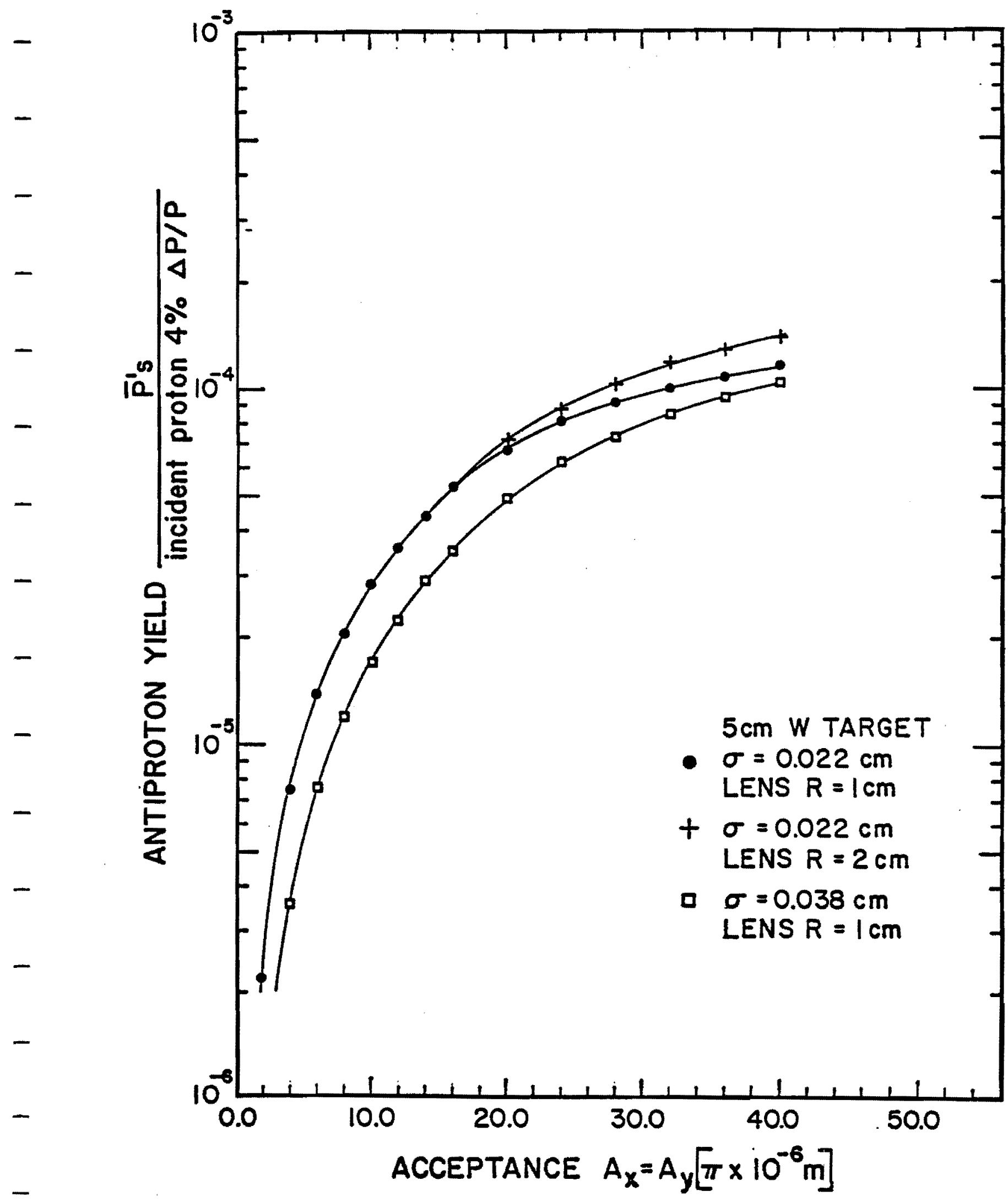

Fig. 3-4 


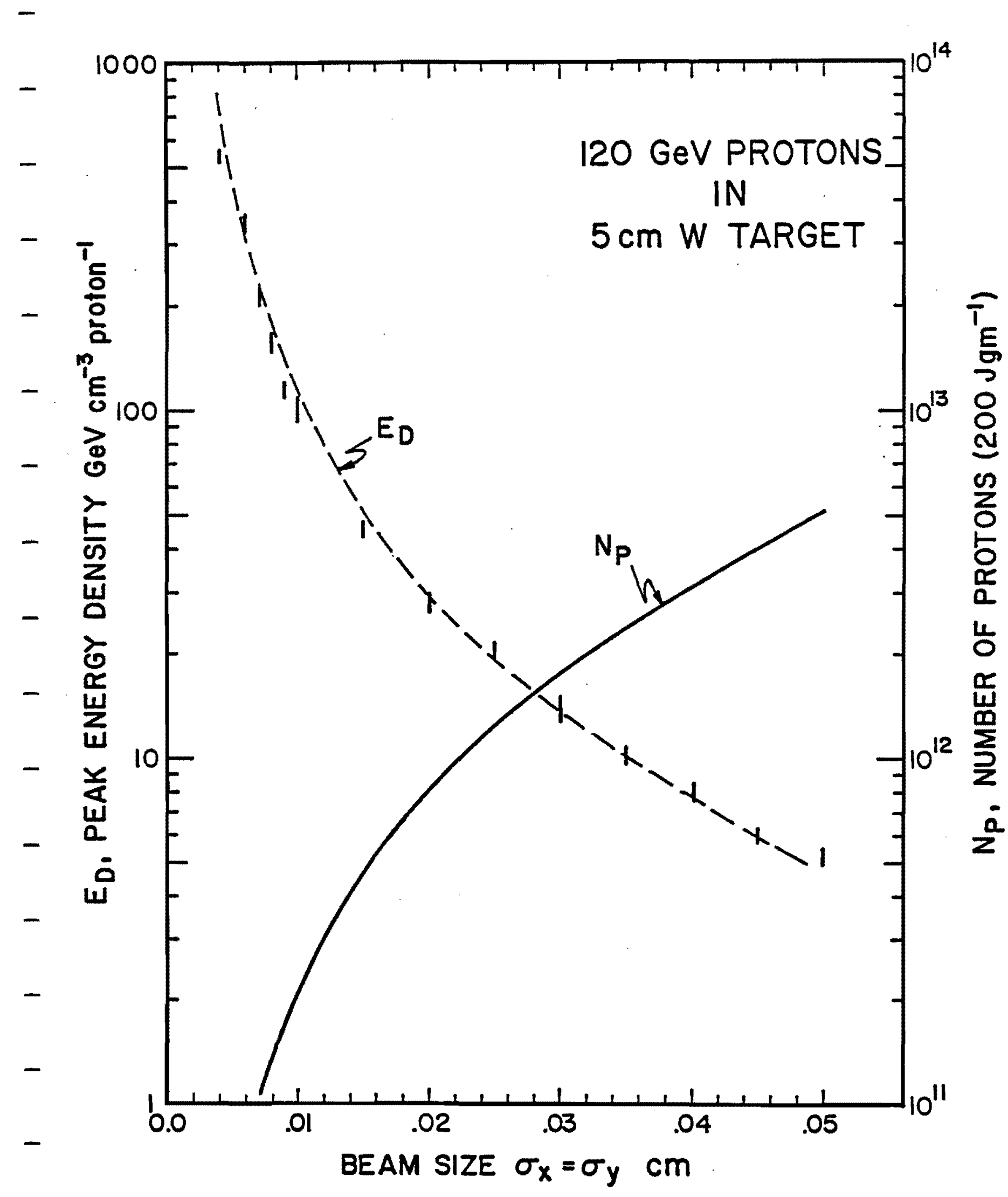

Fig. 3-5 


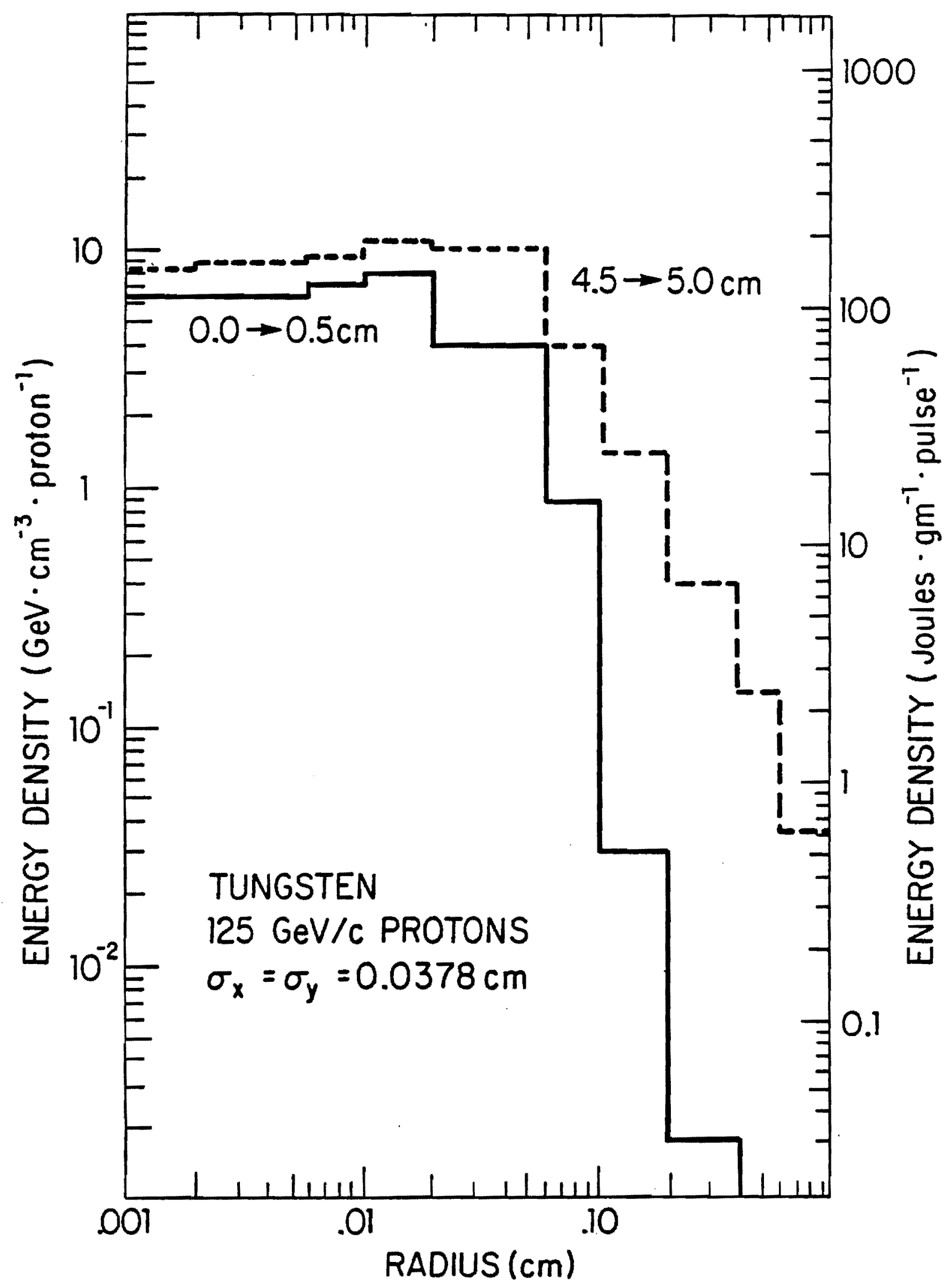

Eig. 3-8 

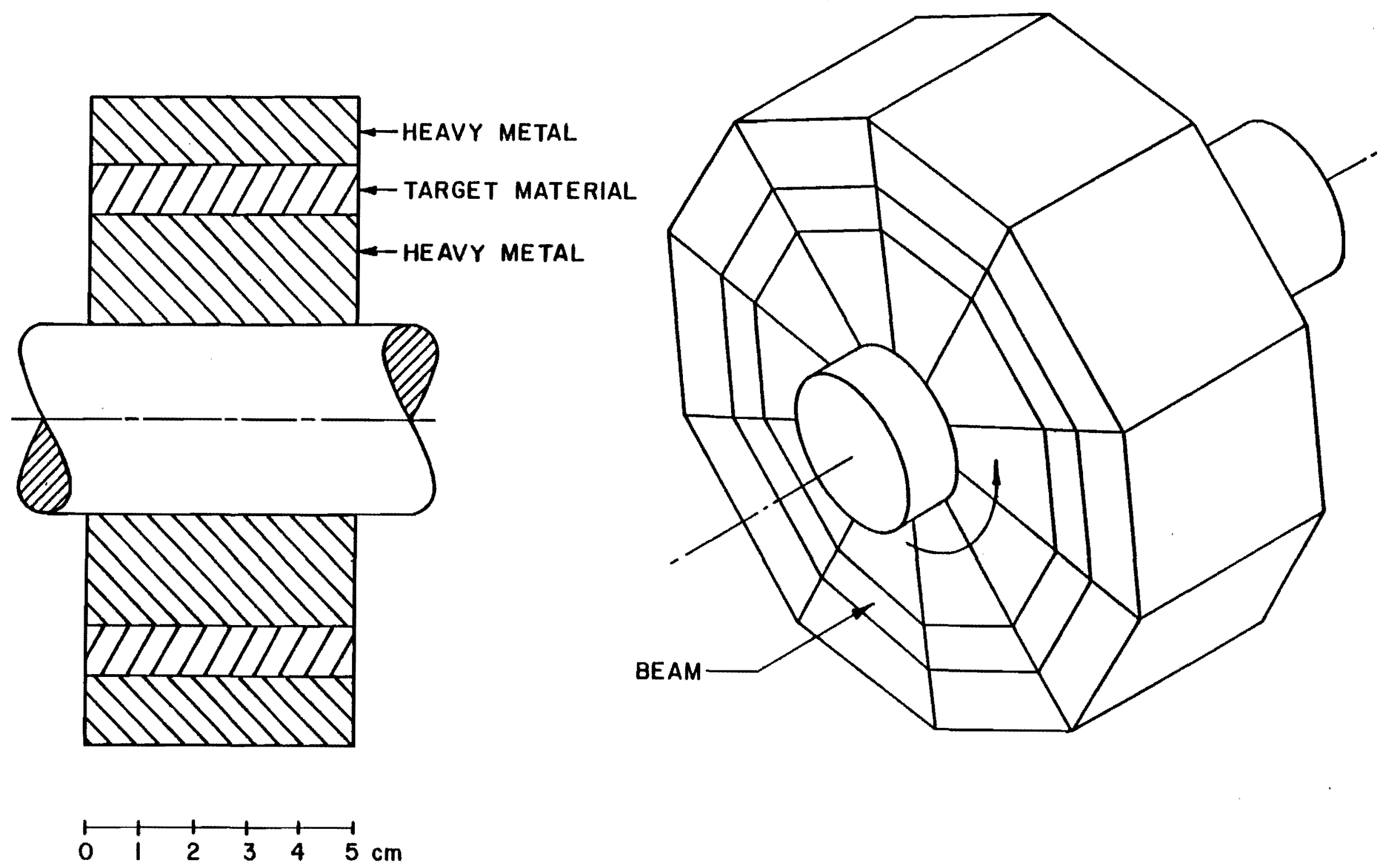
1

1

1

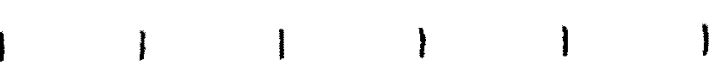

$\begin{array}{lll}1 & 1 & 1\end{array}$

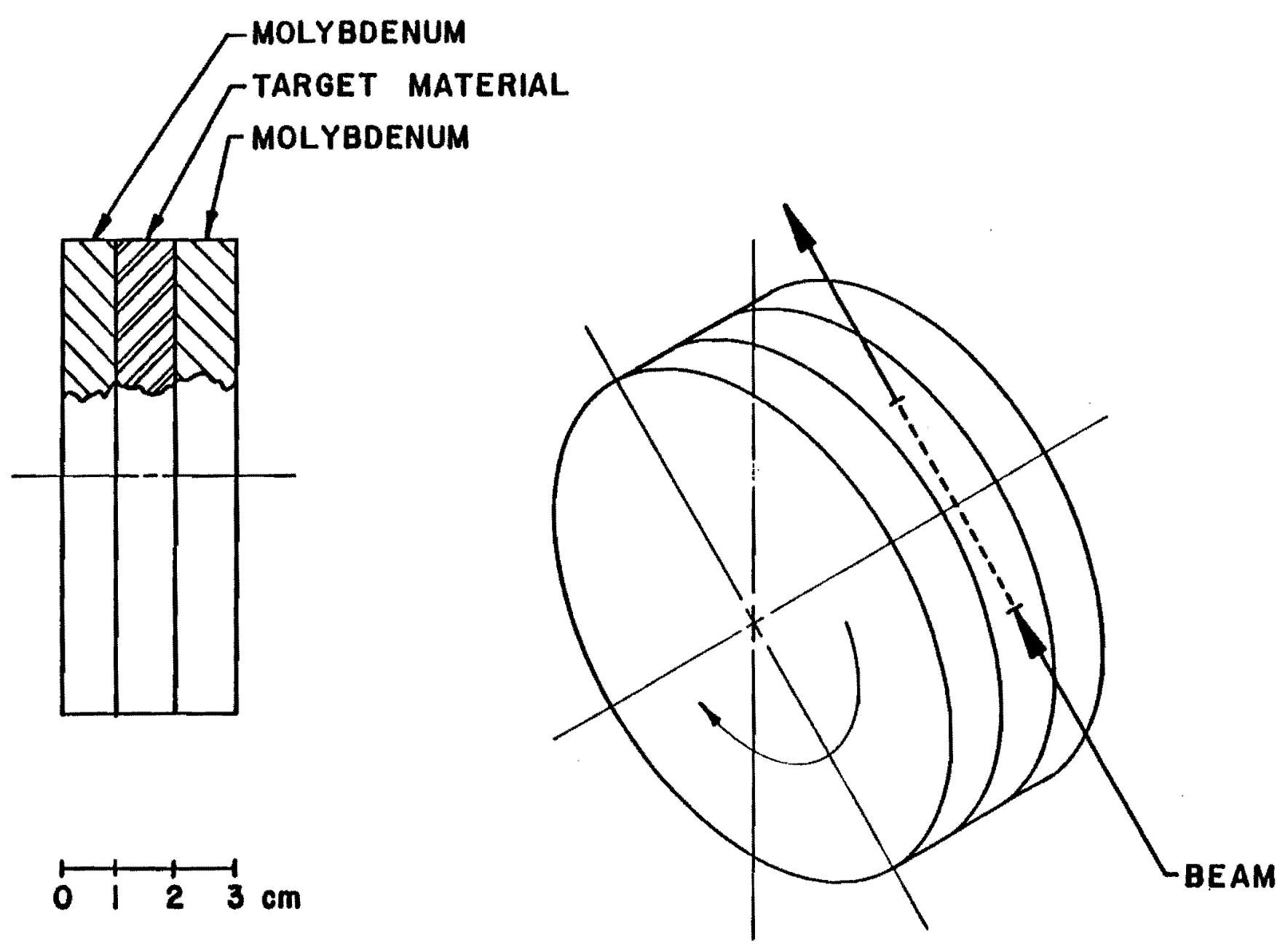

Fig. 3-11 

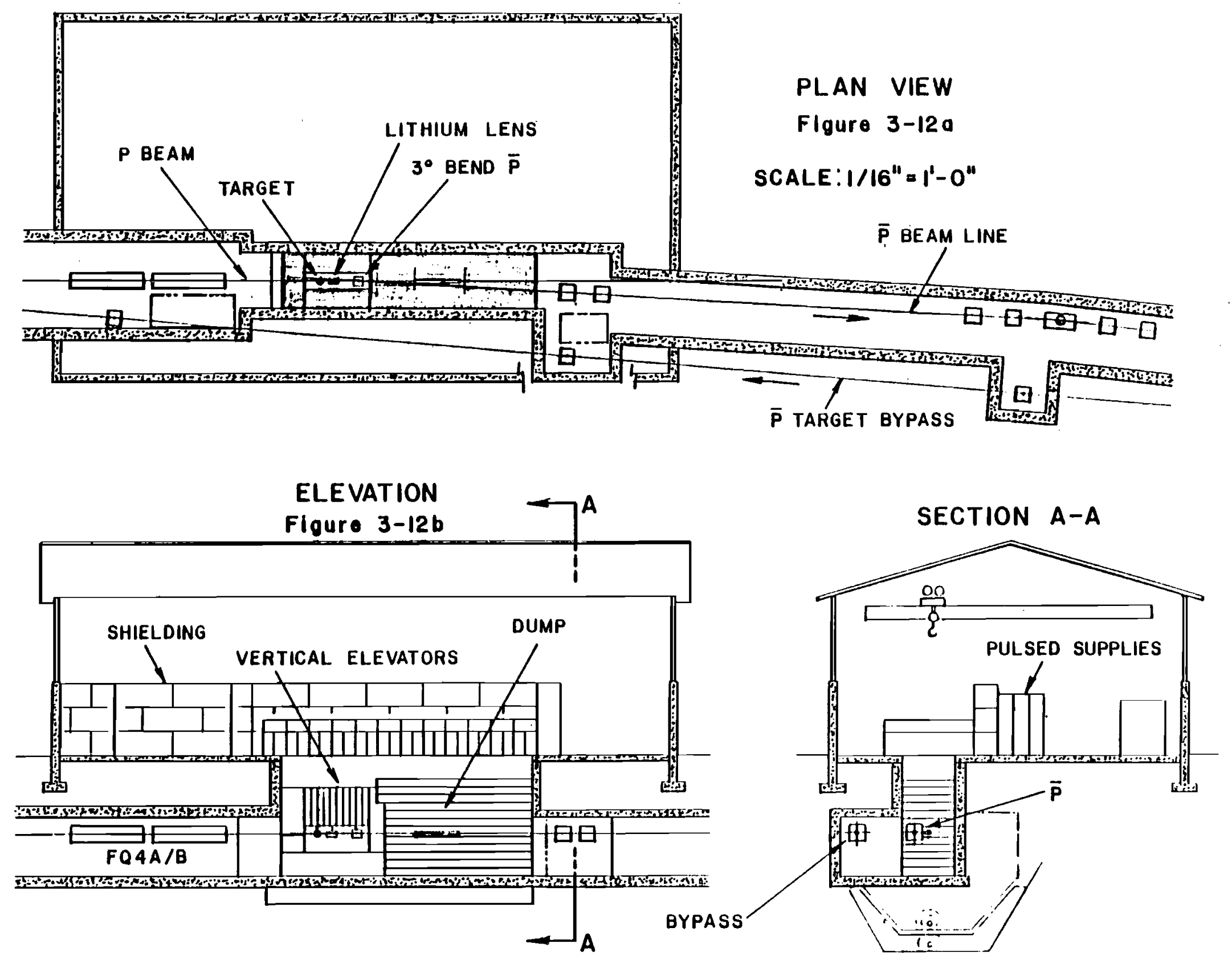


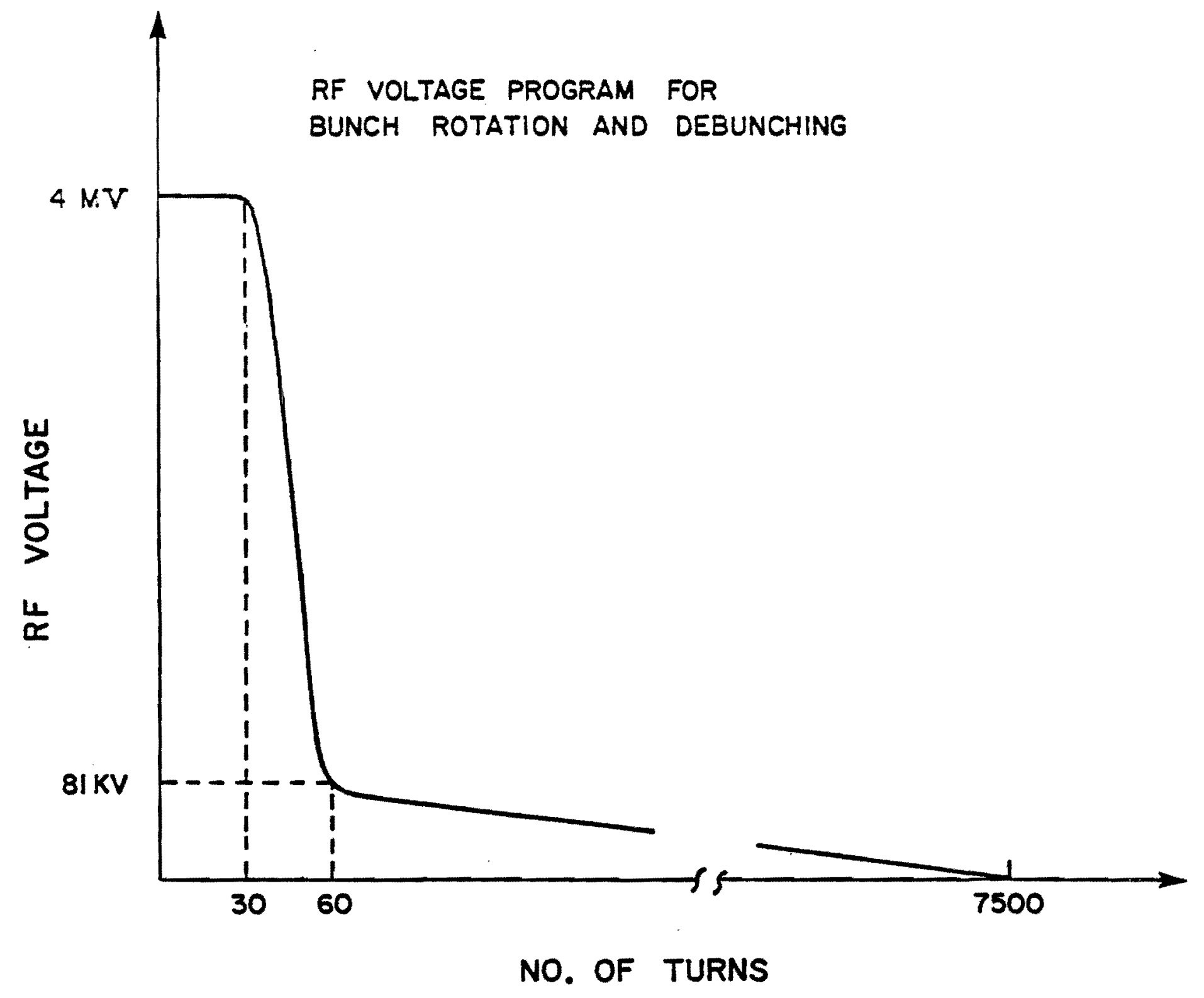

Fig. 4-1 


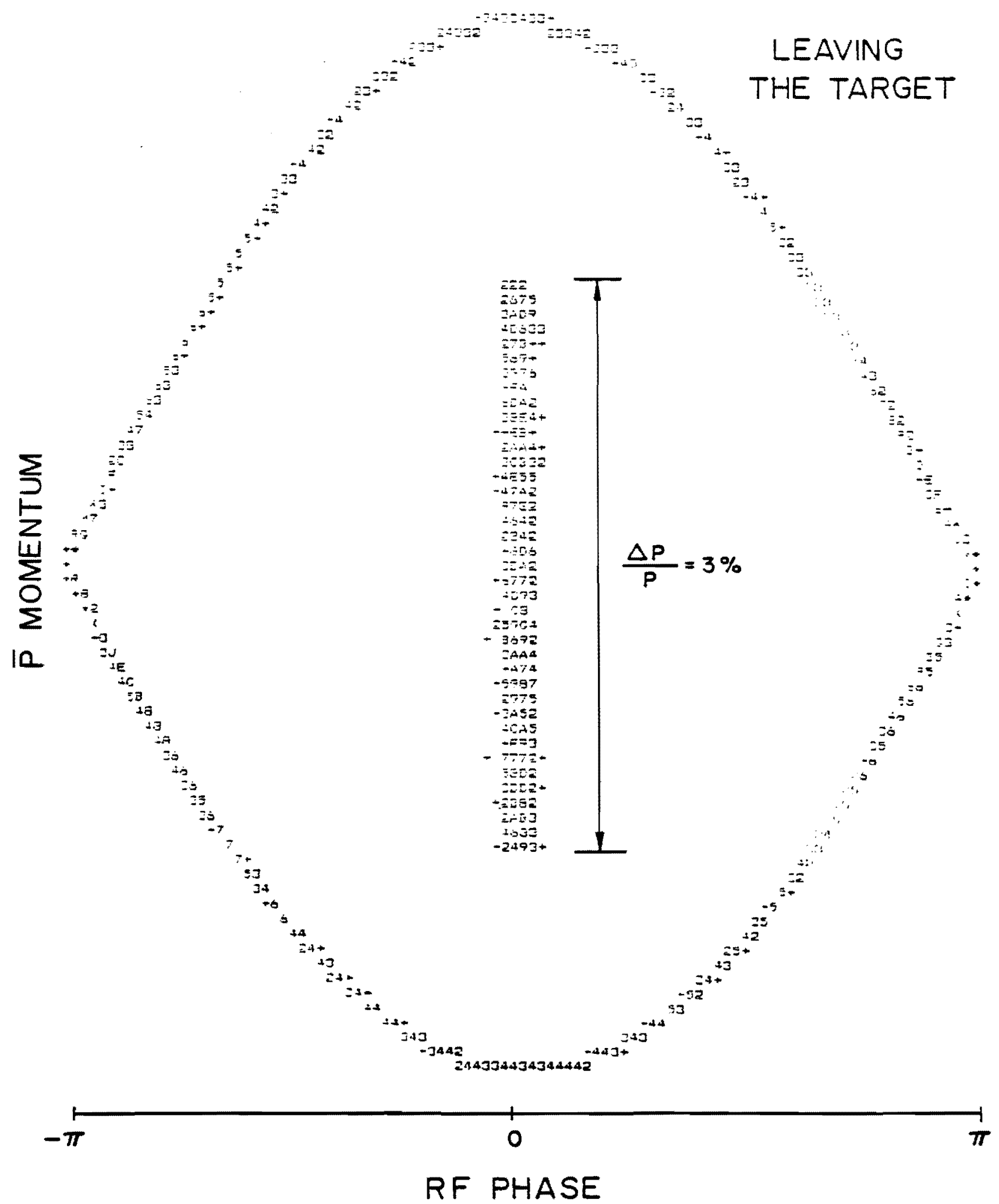

Fig. 4-2 


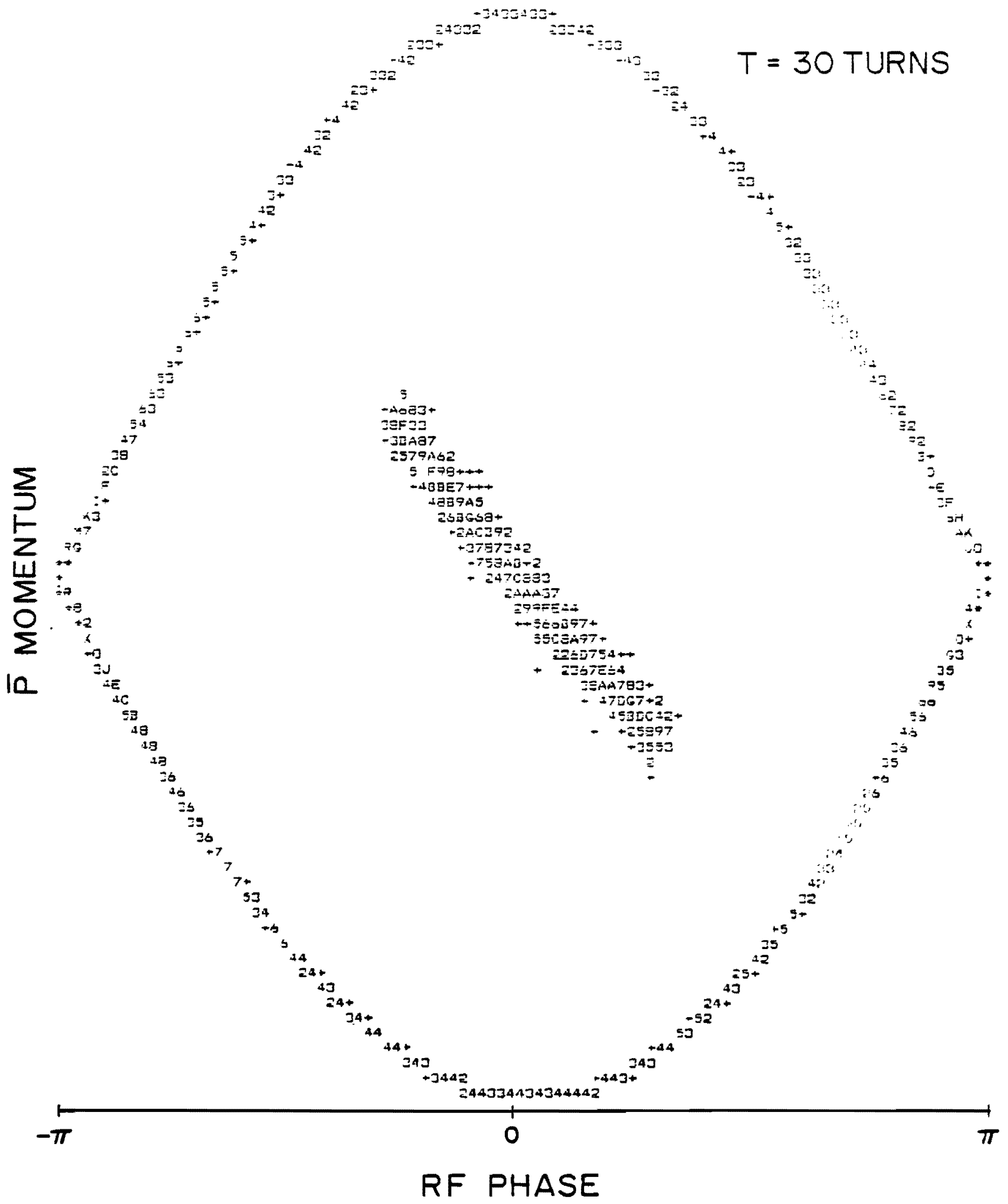

Fig. 4-3 


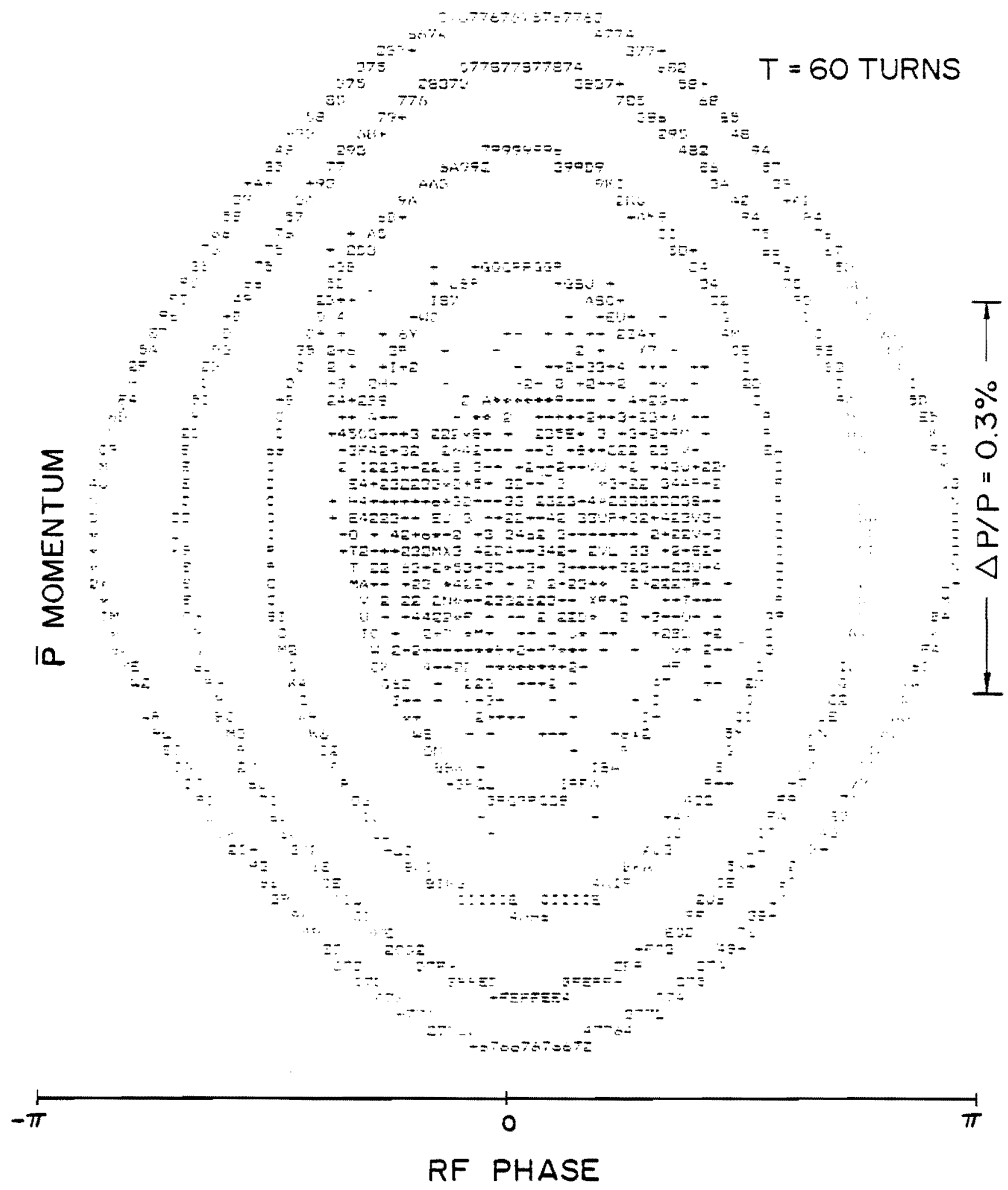

Fig. $4-4$ 


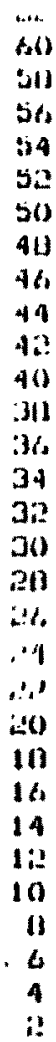

CIIINNI LS 100

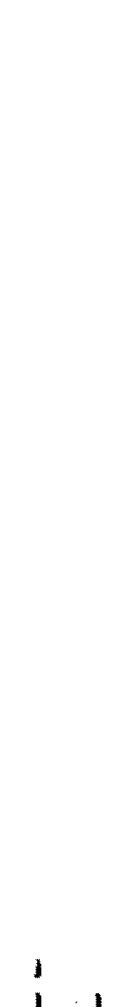

\section{$95 \%$ OF BEAM $\frac{\Delta P}{P}=0.2 \%$ FULL WIDTH}

100

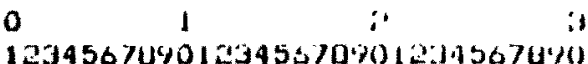

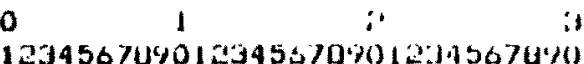

CONIENTS

1

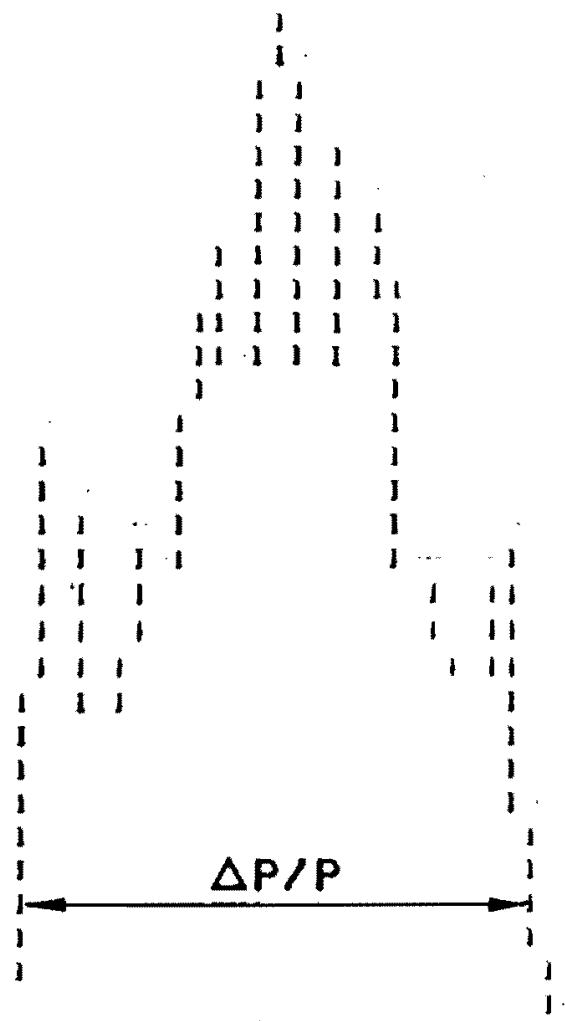

$1 \cdots 11$

LOW-EDGE 1000

BQbatน

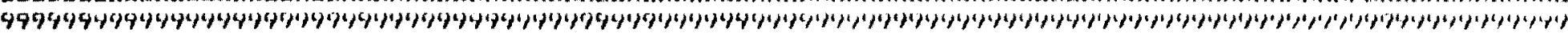

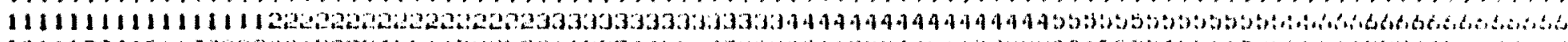

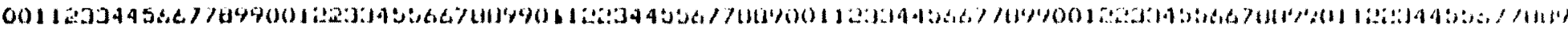

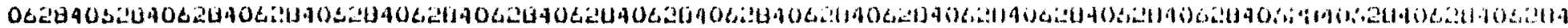


Debunched Beam Spread

vS.

Initial Beam Spread for $V=4 M V$ and $a$ Final $V=5 \mathrm{KV}$

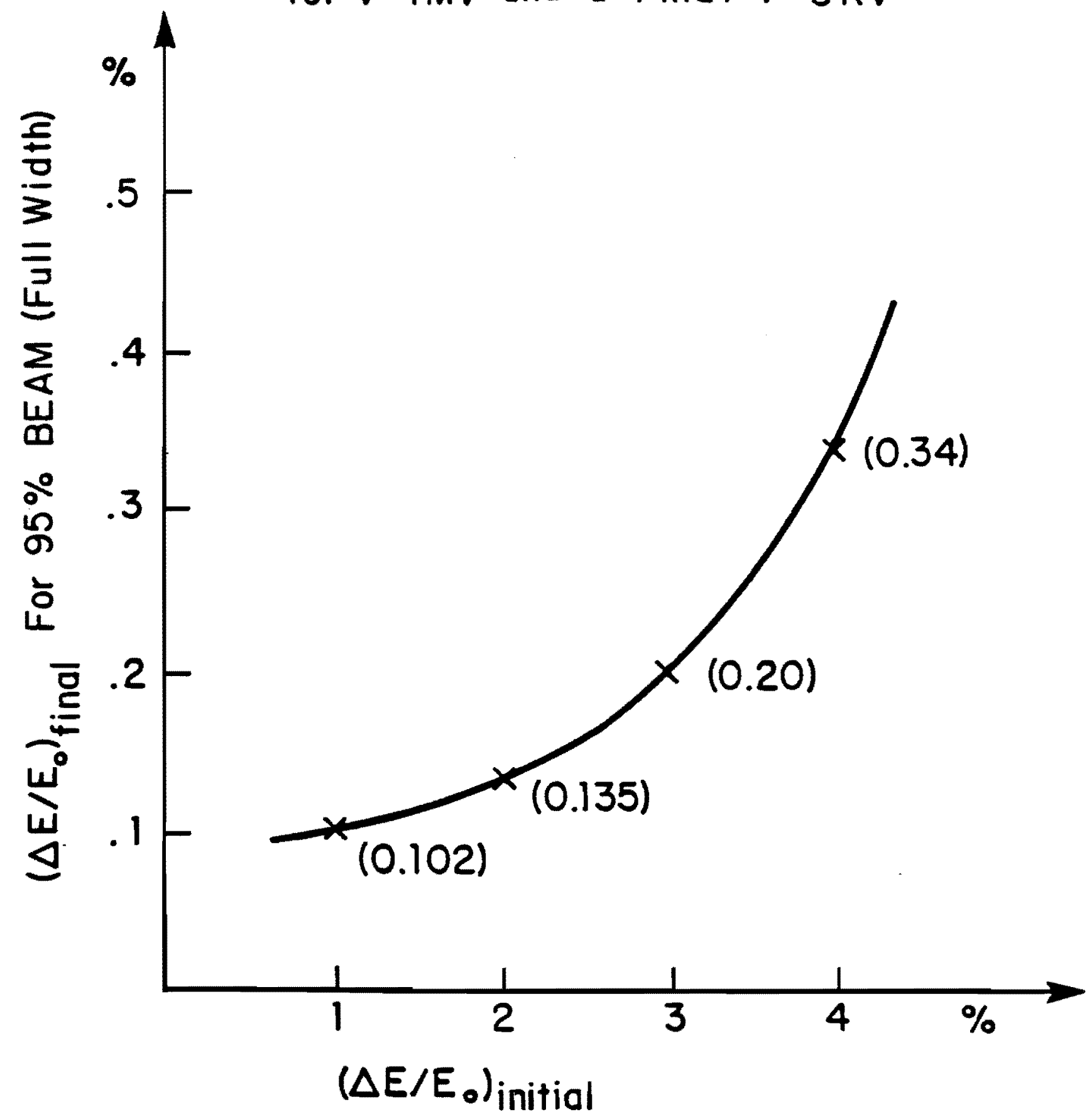

Fig. 4-6 

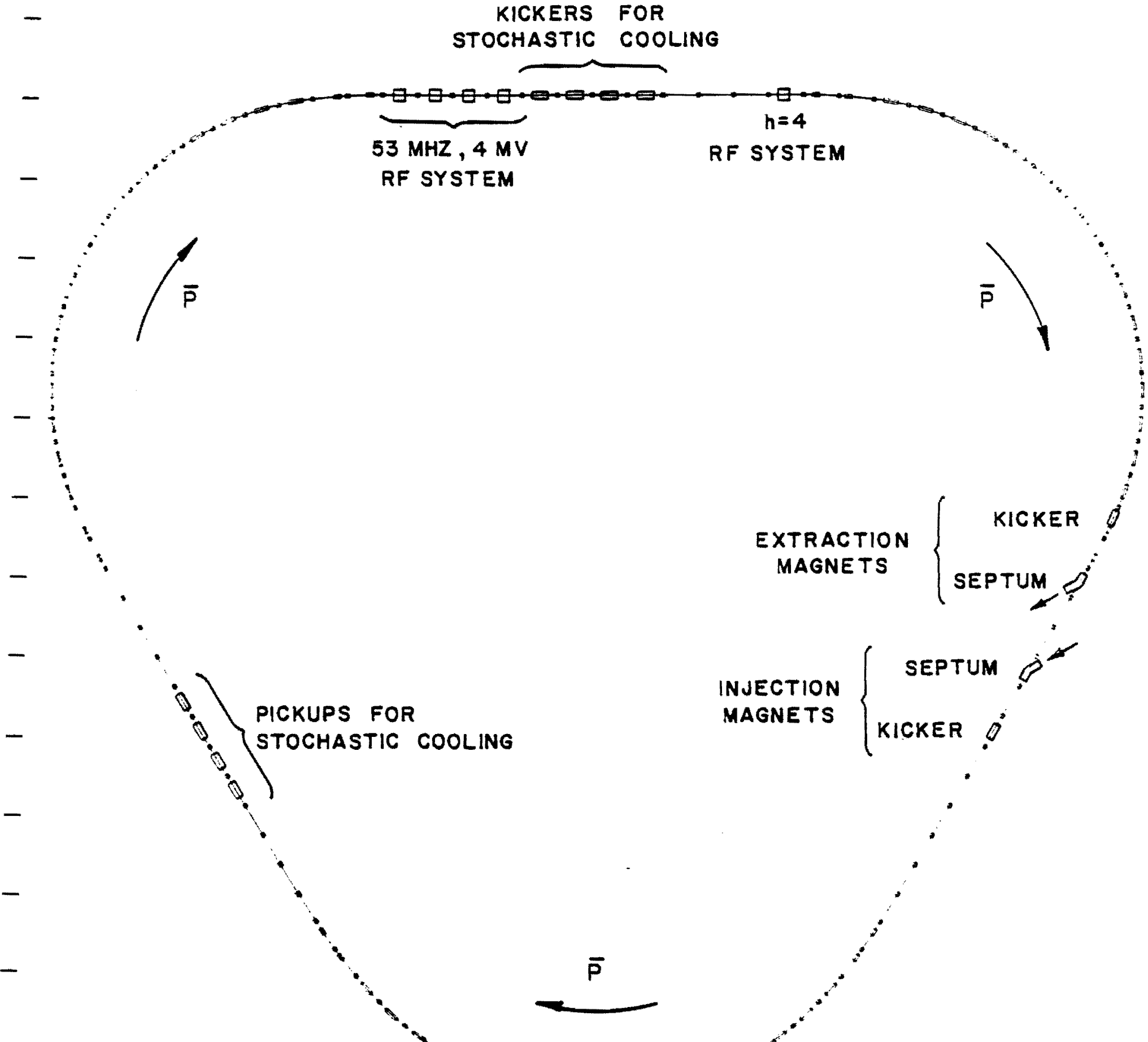
DEBUNCHER REGULAR CELL

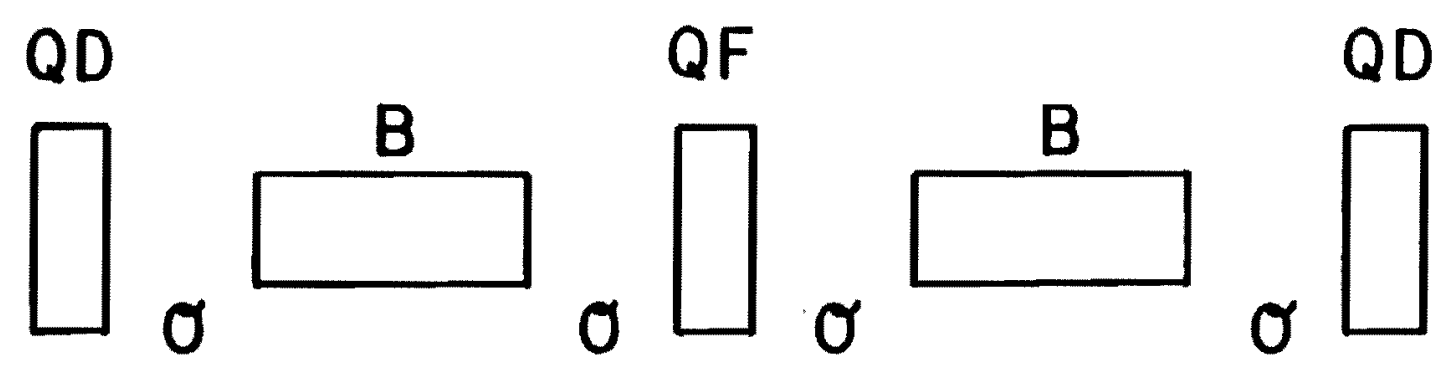

$$
\begin{aligned}
B & =15.28 \mathrm{KG}, 2.258 \mathrm{~m} & \sigma & =1.227 \mathrm{~m} \\
Q F & =2 \text { feet } & B^{\prime} / B_{\rho} & =0.580 \mathrm{~m}^{-2} \\
Q D & =2 \text { feet } & B^{\prime} / B_{\rho} & =0.560 \mathrm{~m}^{-2}
\end{aligned}
$$

TOTAL LENGTH $=10.6437 \mathrm{~m}$ 
DISPERSION-KILLER SECTION
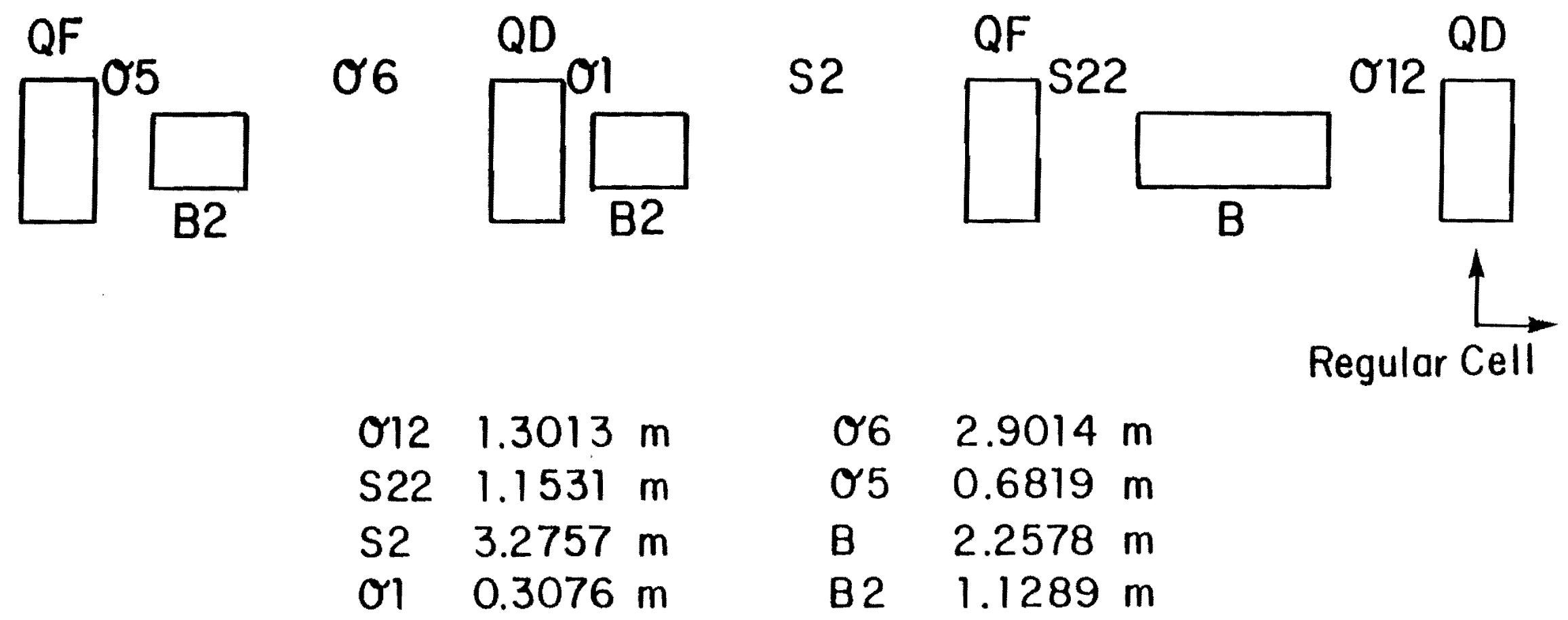

$06 \quad 2.9014 \mathrm{~m}$ o5 $0.6819 \mathrm{~m}$

B $2.2578 \mathrm{~m}$

B2 $1.1289 \mathrm{~m}$ 


\section{TYPICAL CELL FOR A LONG STRAIGHT SECTION}

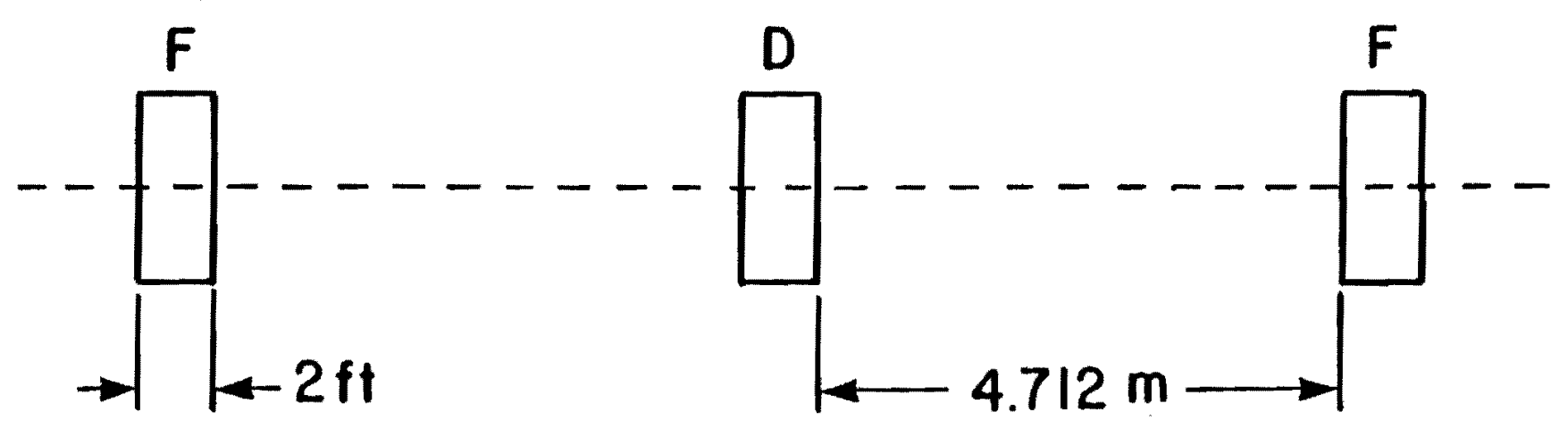

TOTAL LENGTH Including Quads $=66 \mathrm{~m}$ 


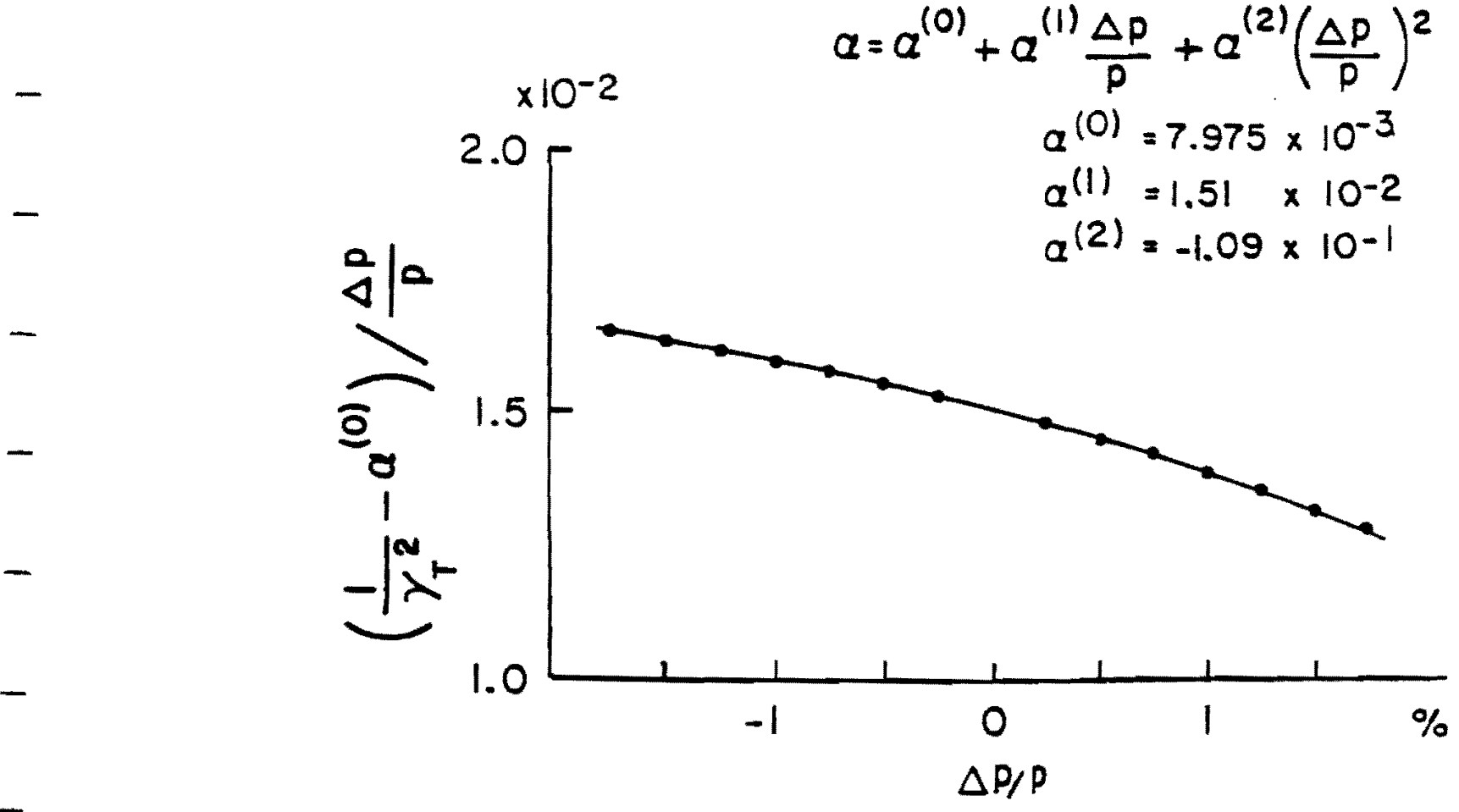

Fig. 4-11

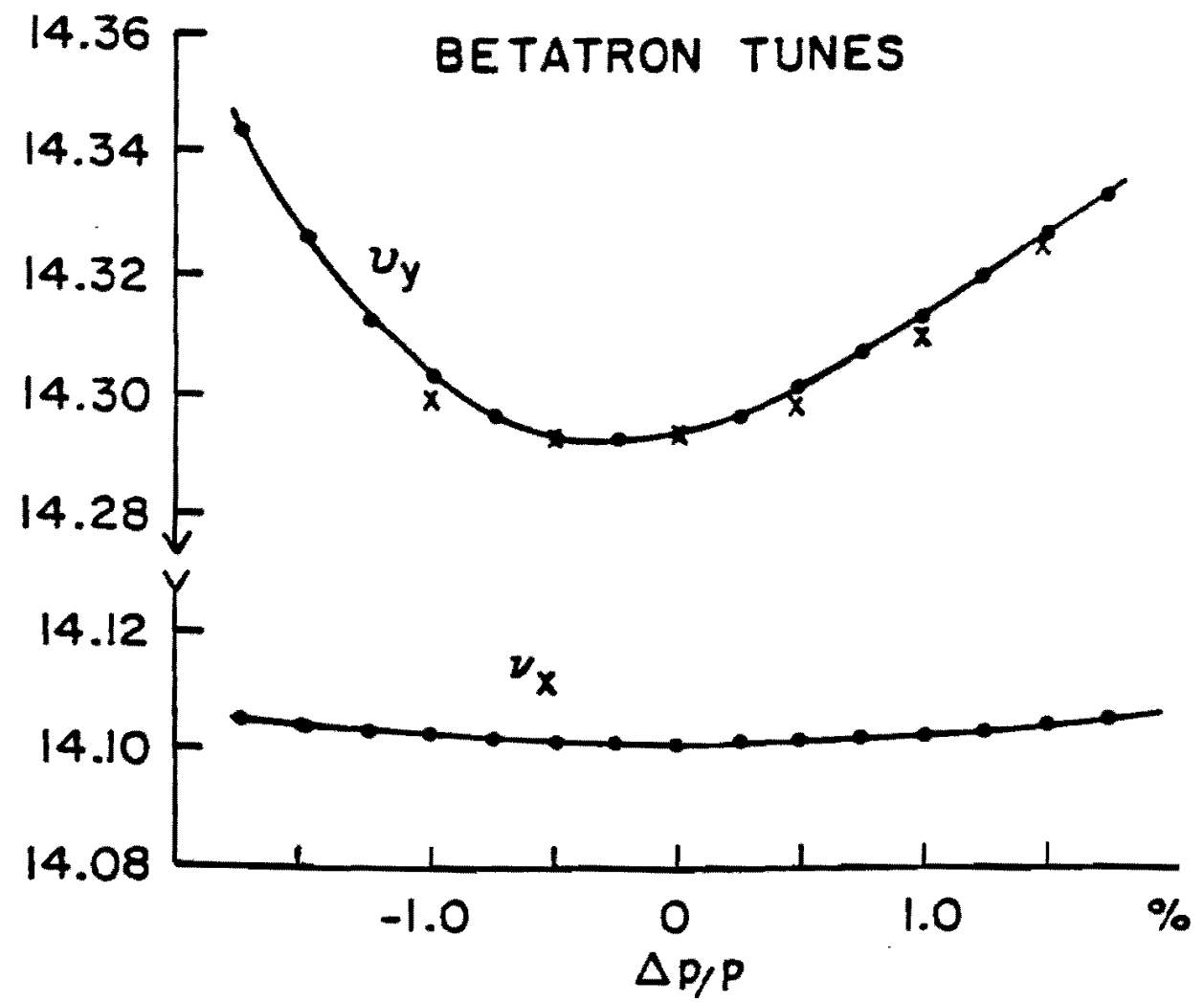

Fig. 4-12 


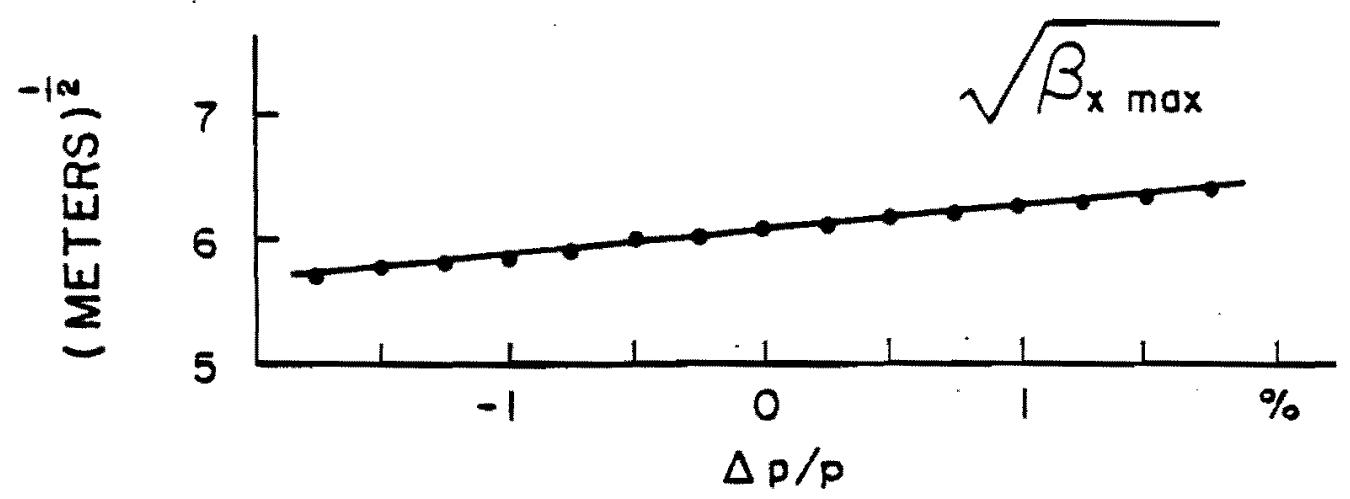

Fig. 4-13a

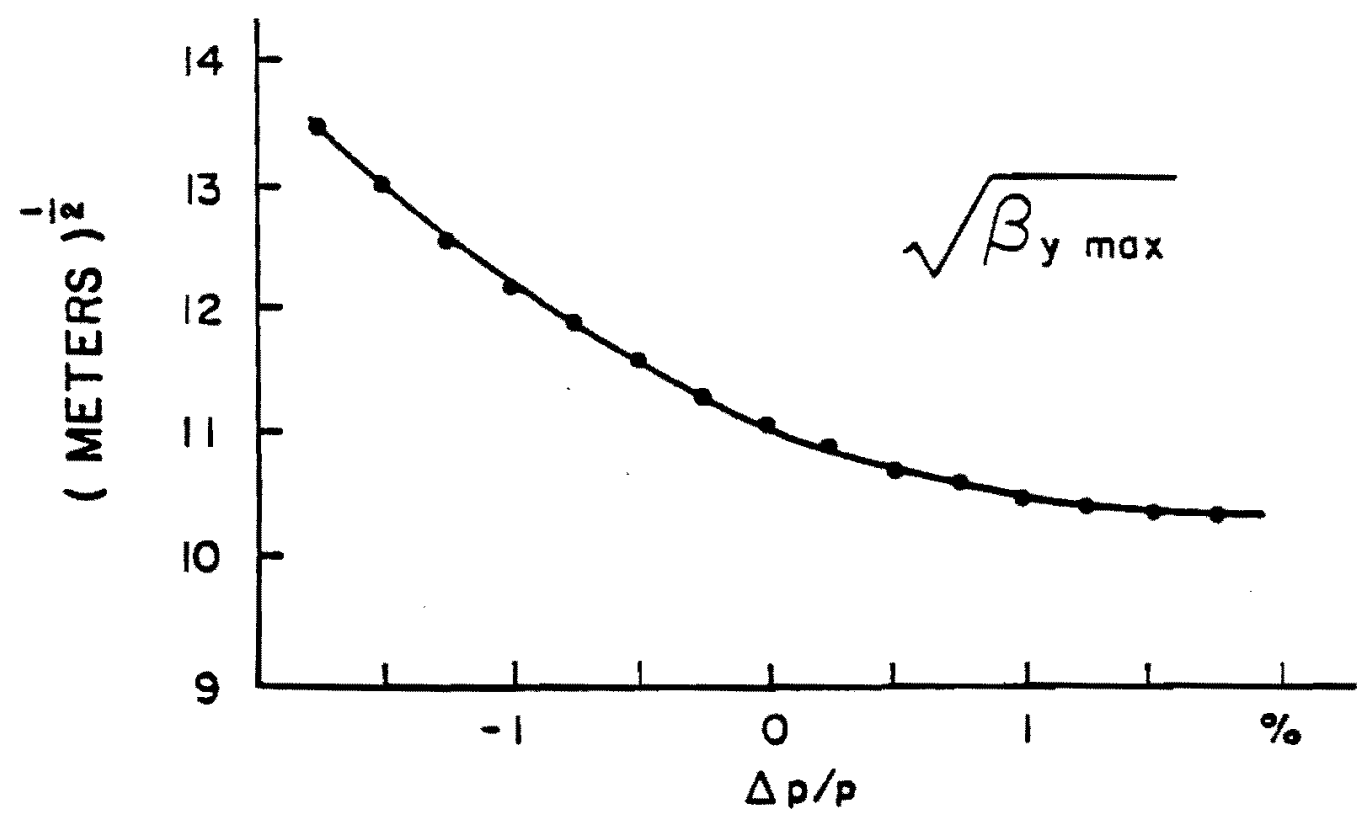

Fig. 4-13b

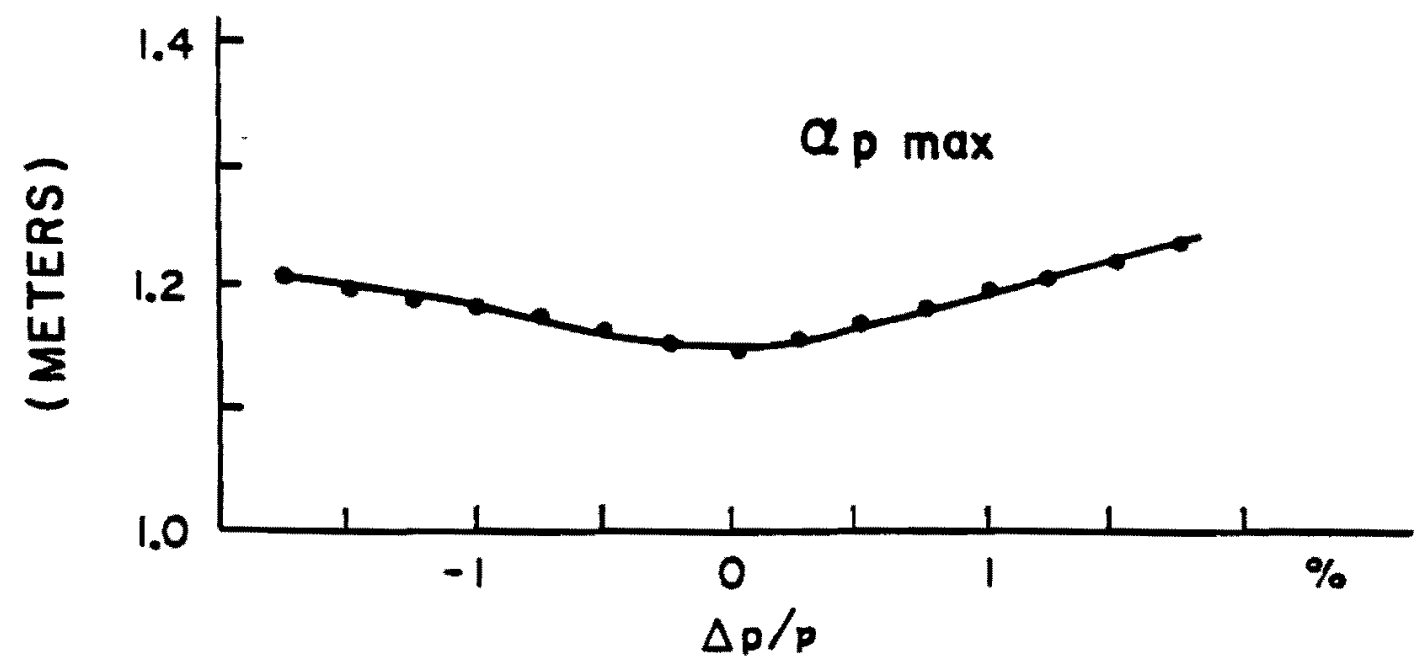

Fig. $4-13 c$ 
LATTICE FUNCTIONS ALONG ONE HALF-PERIOD

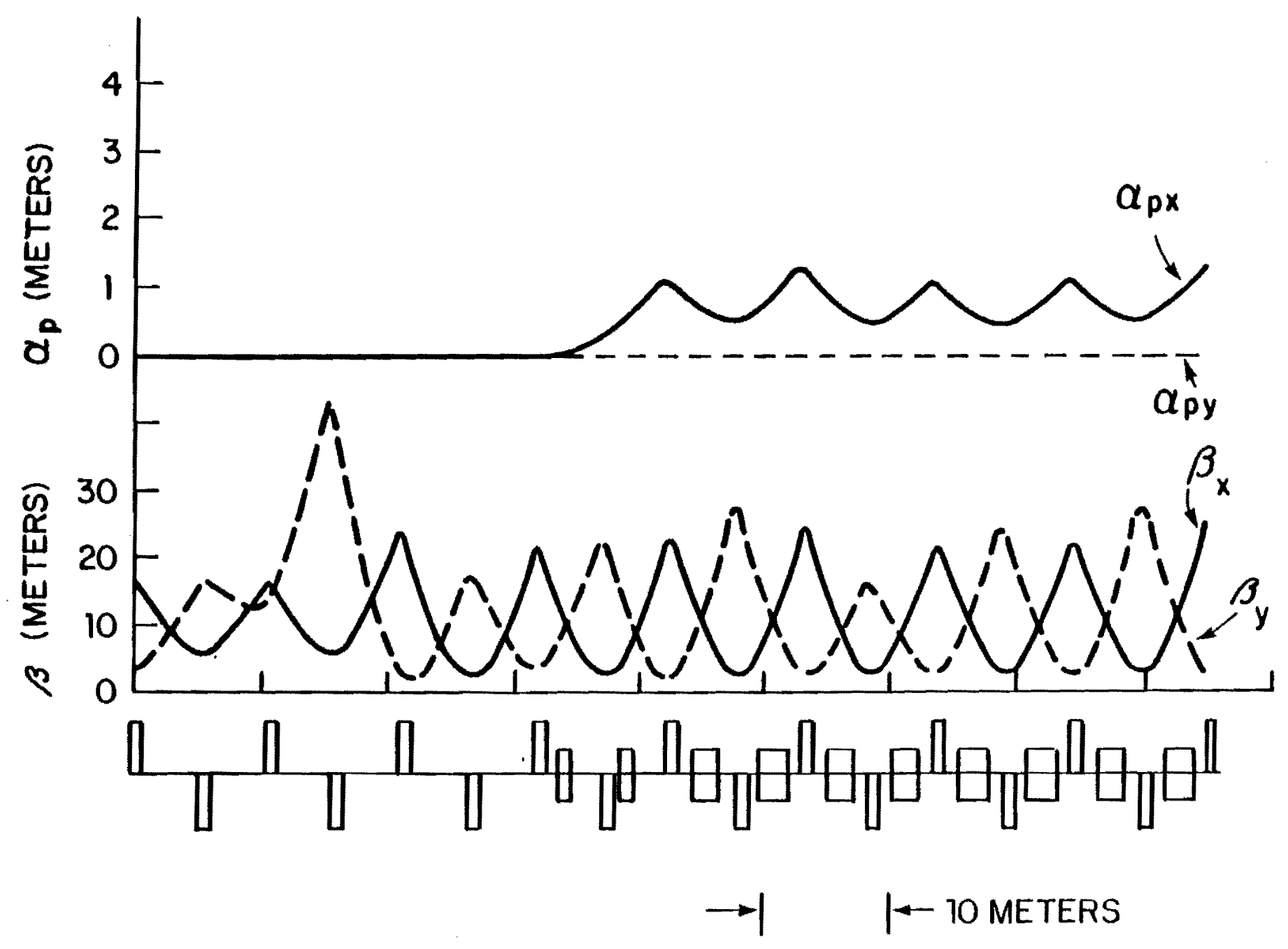

Fig. 4-14 


\section{INJECTION AND EXTRACTION IN THE DEBUNCHER}

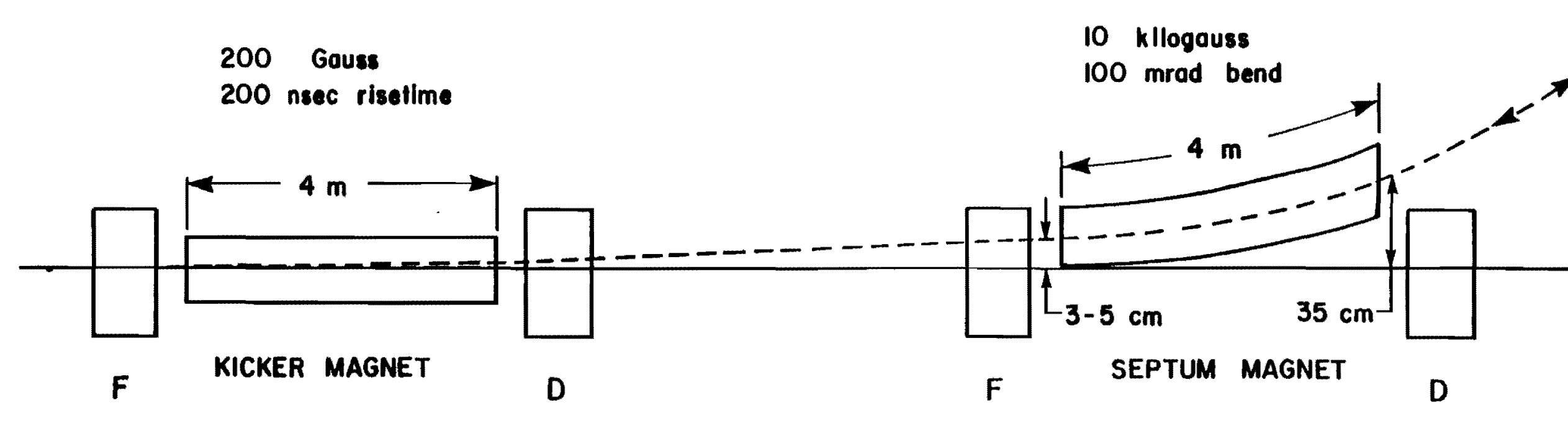

ALLOWANCE FOR

$$
\begin{aligned}
& \varepsilon_{v}=\varepsilon_{H}=20 \pi \mathrm{mm} \cdot \mathrm{mrad} \\
& \Delta p / p= \pm 2 \%
\end{aligned}
$$




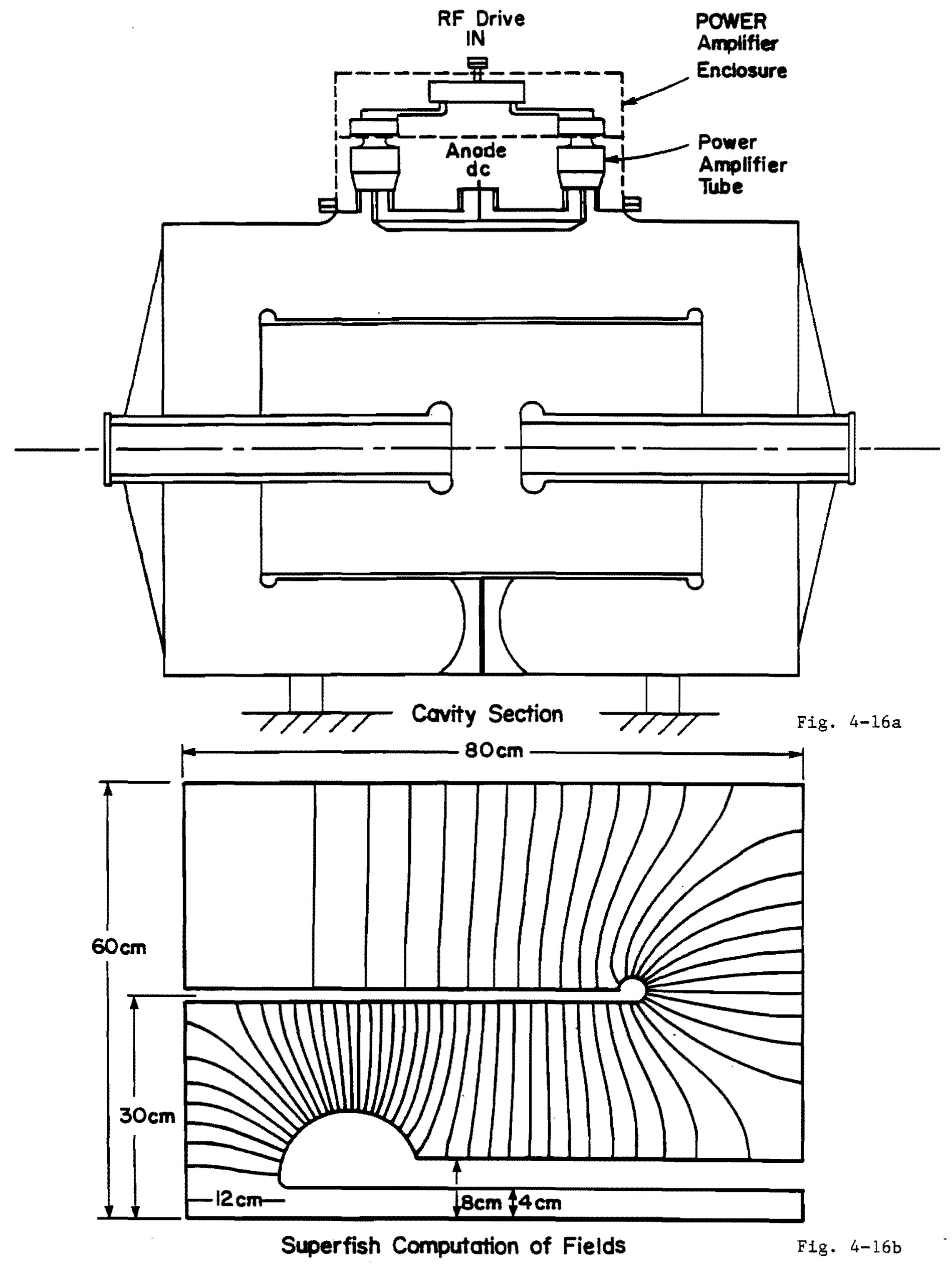



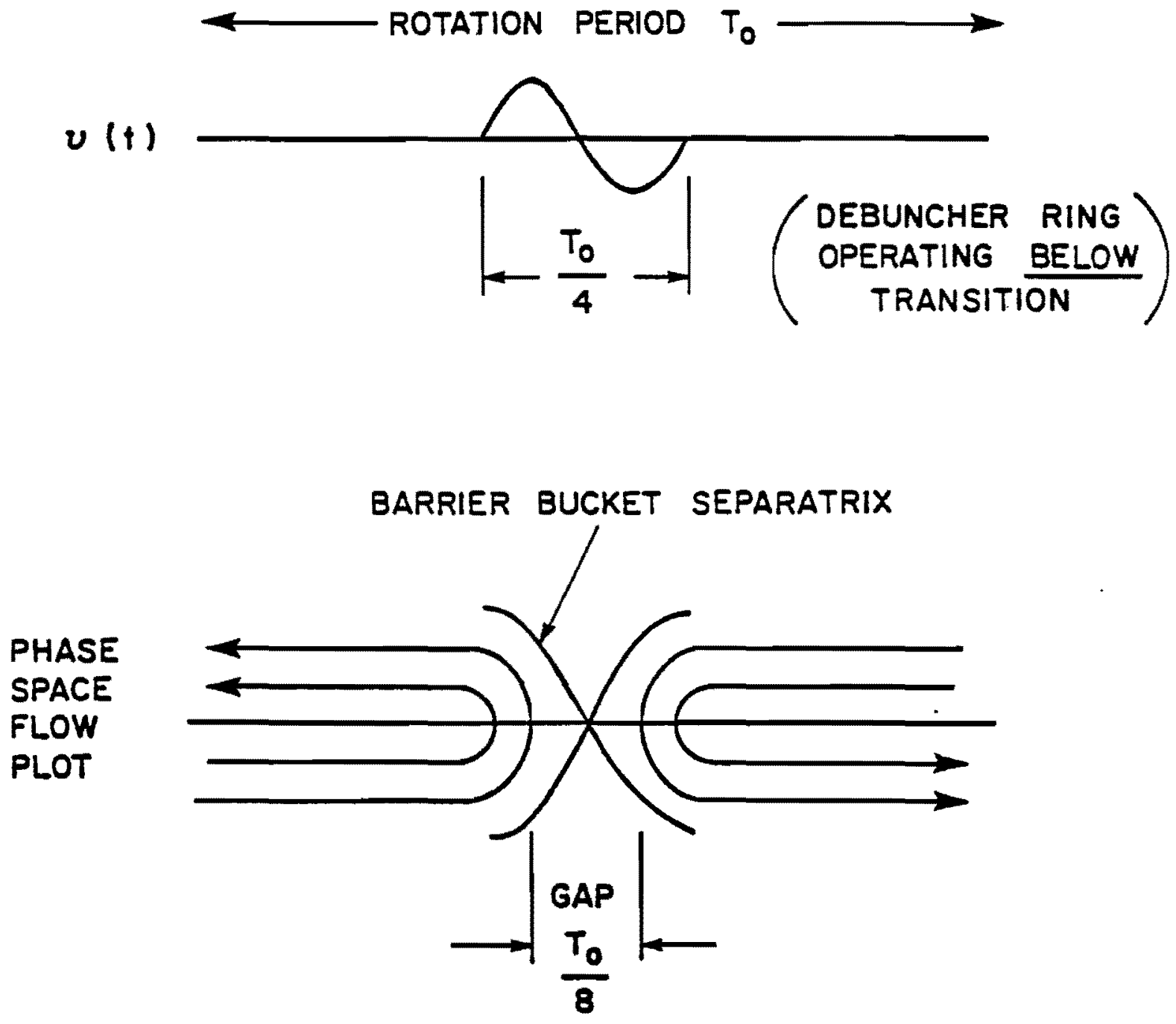

EQUIVALENT CURRENT PULSE

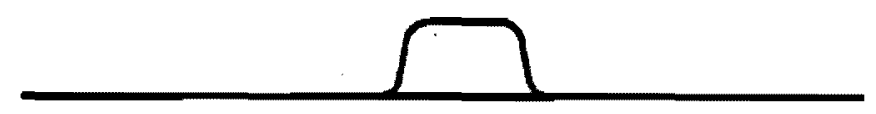




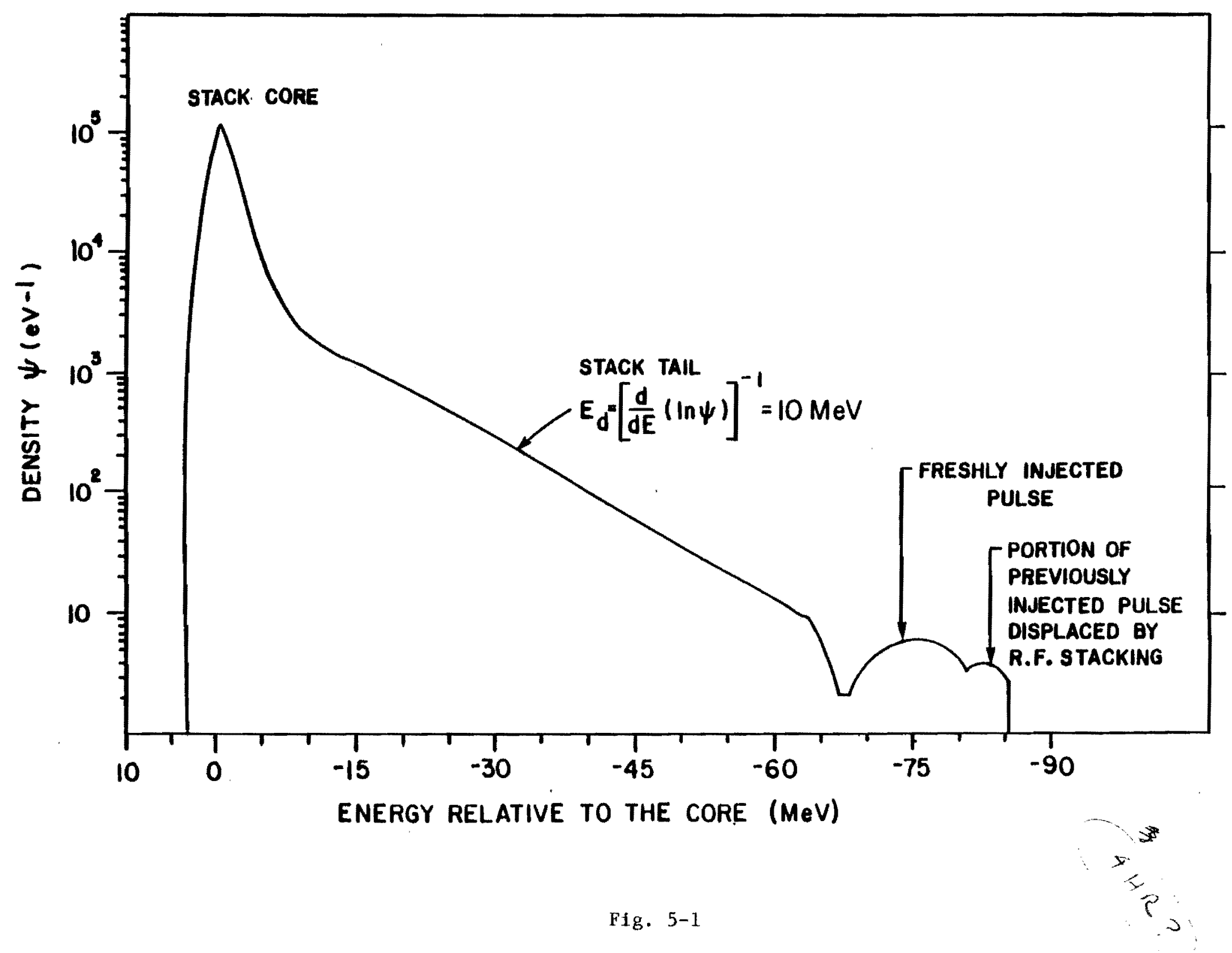



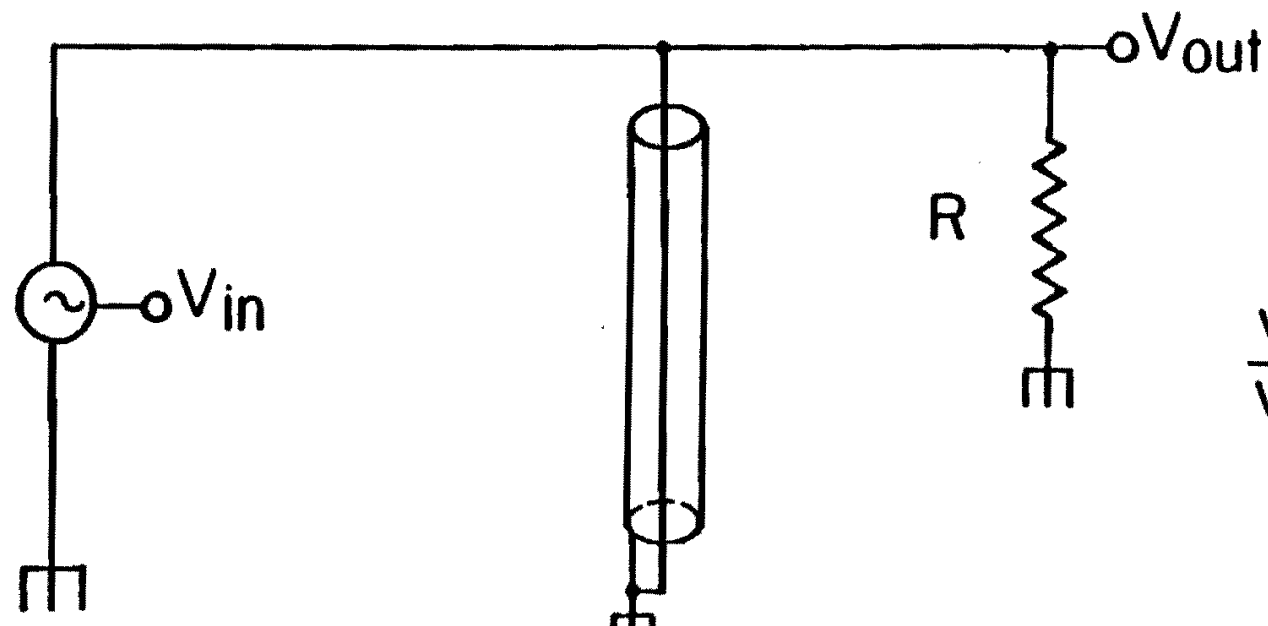

$\frac{V_{\text {out }}}{V_{\text {in }}}=\frac{Z_{L} / R}{\operatorname{coth}(\alpha \ell+j \beta \ell)+Z_{L} / R}$

Current

Source

$I=\frac{V_{\text {in }}}{R}$

\section{Shorted}

Transmission line

Impedance $=Z_{L}$

Loss $=\alpha \ell$
Phase $=\beta \ell=\frac{2 \pi \ell f}{v}$

$f=$ freguency

$v=$ phase velocity 
Stack Tail Momentum Cooling System I- $2 \mathrm{GHz}$
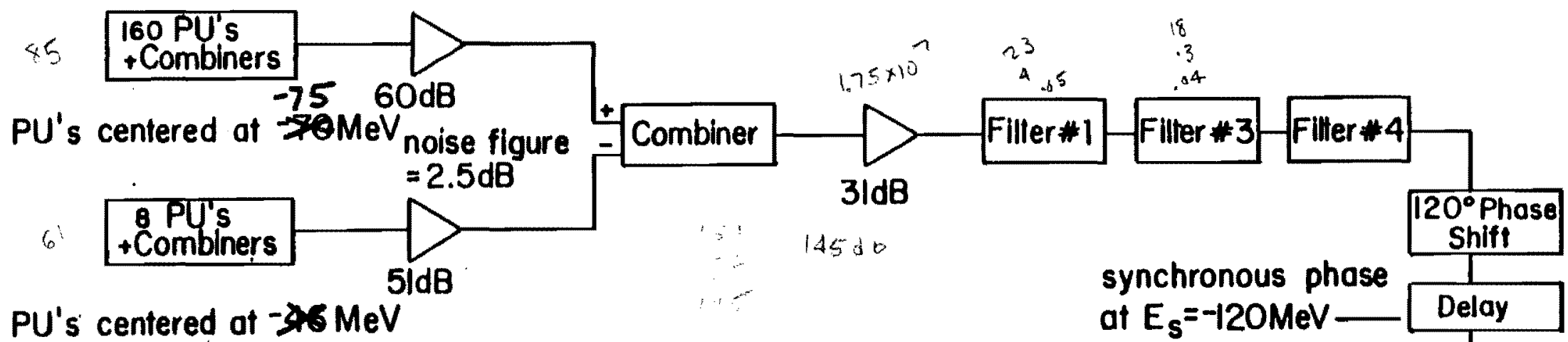

2.10

PU's centered of $-\$ 16 \mathrm{MeV}$

$-51$
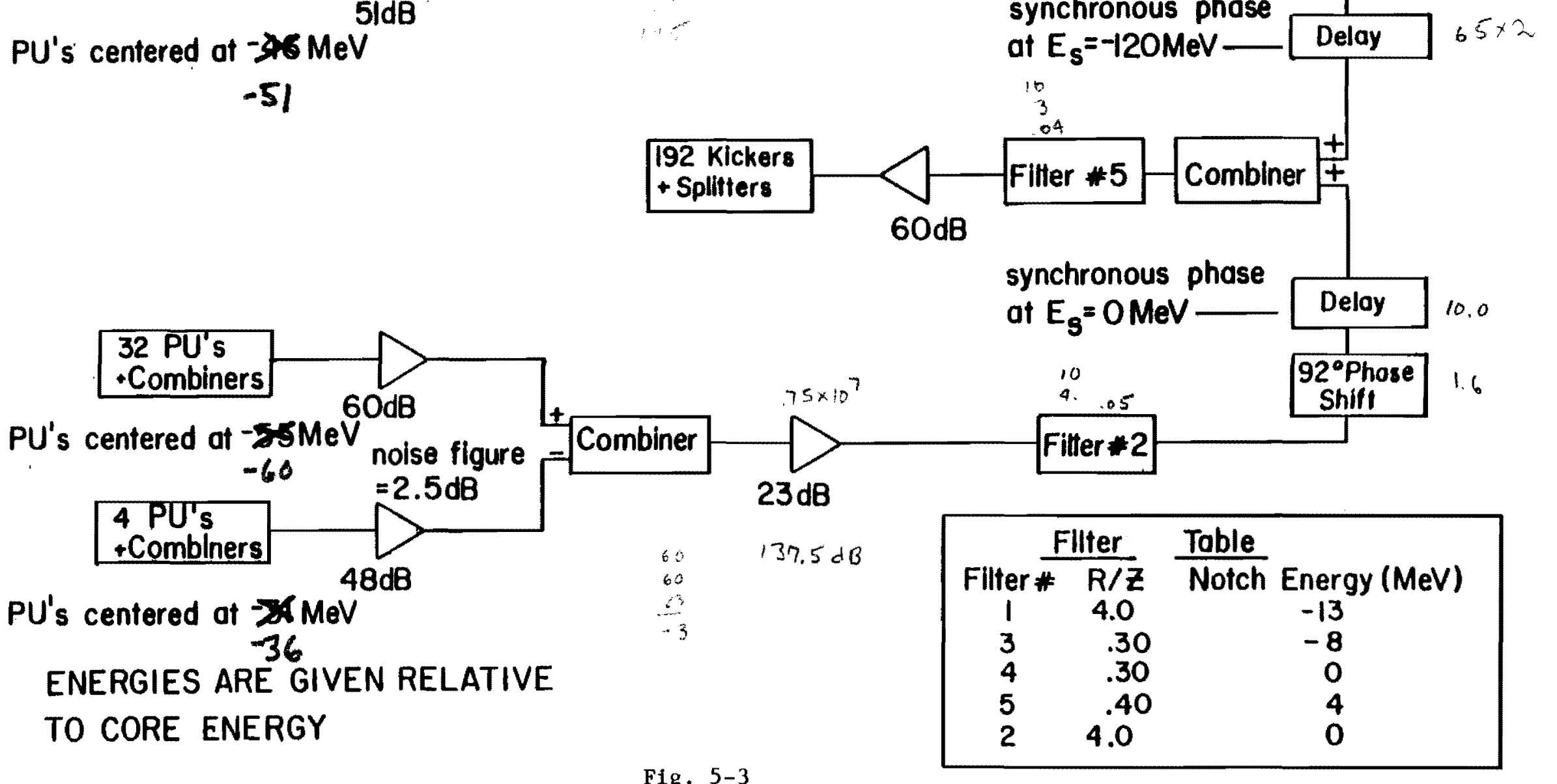


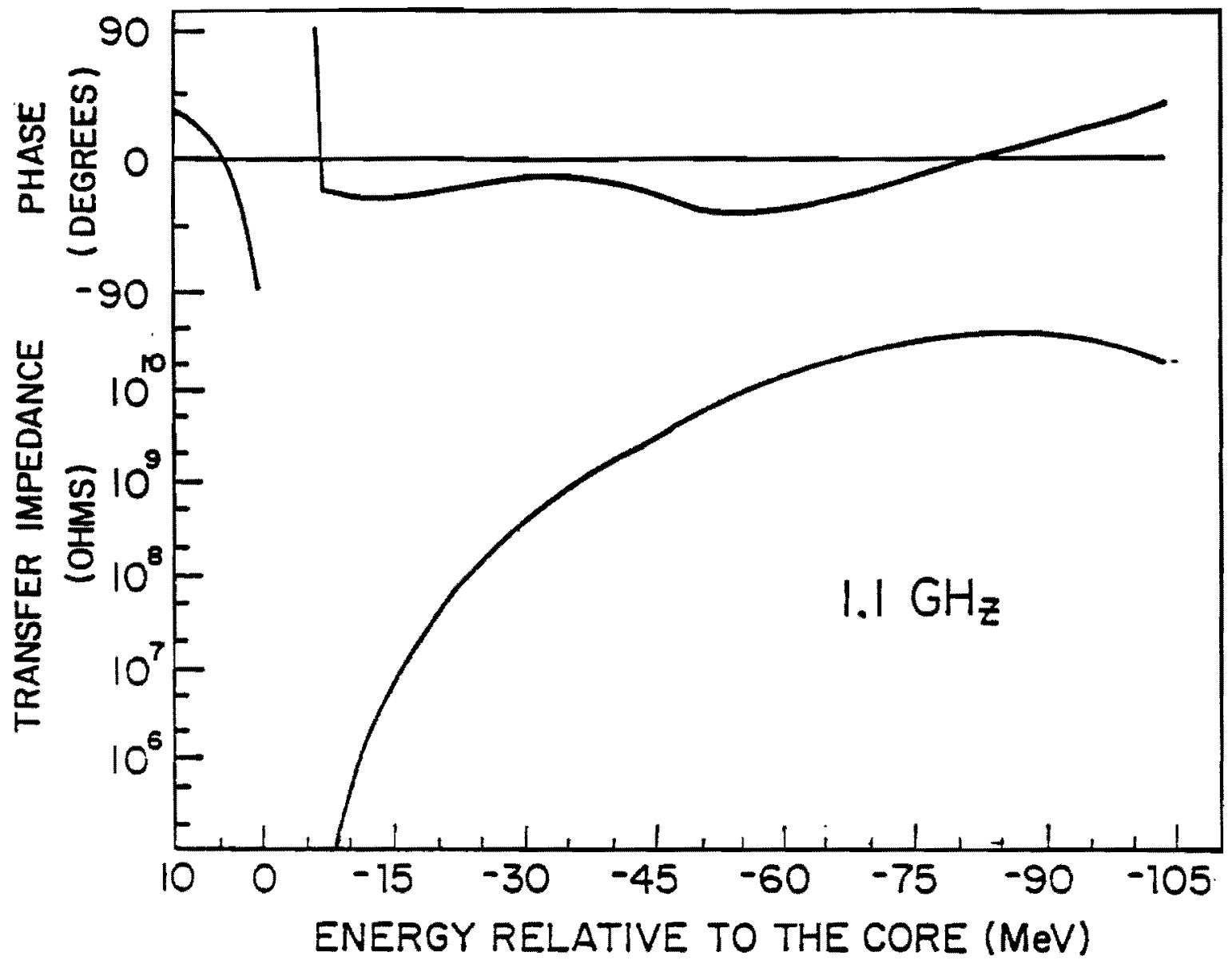

Fig. 5-4a

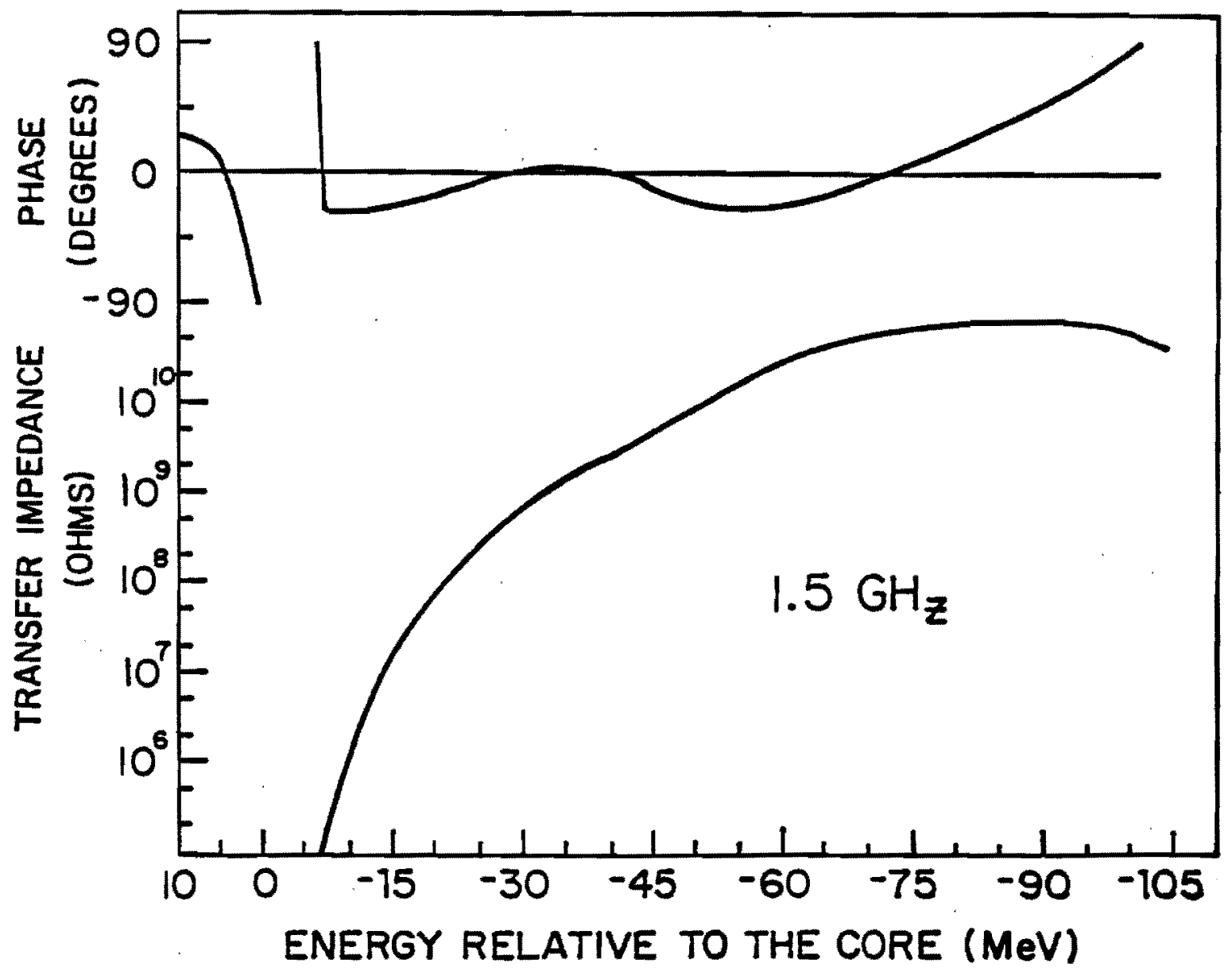

Fig. 5-4b 


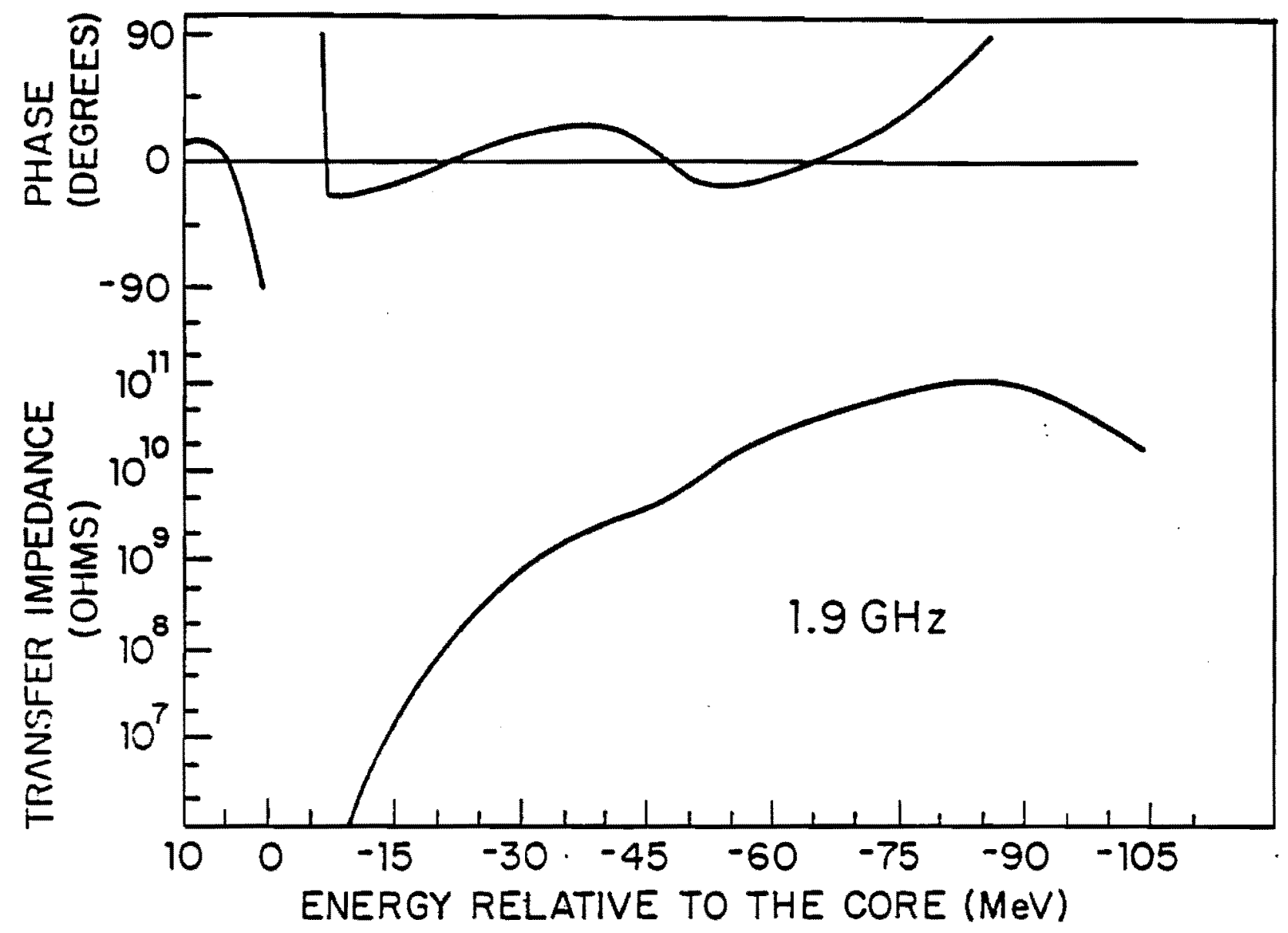

Fig. $5-4 c$

Core Momentum Cooling System $2-4 \mathrm{GHz}$

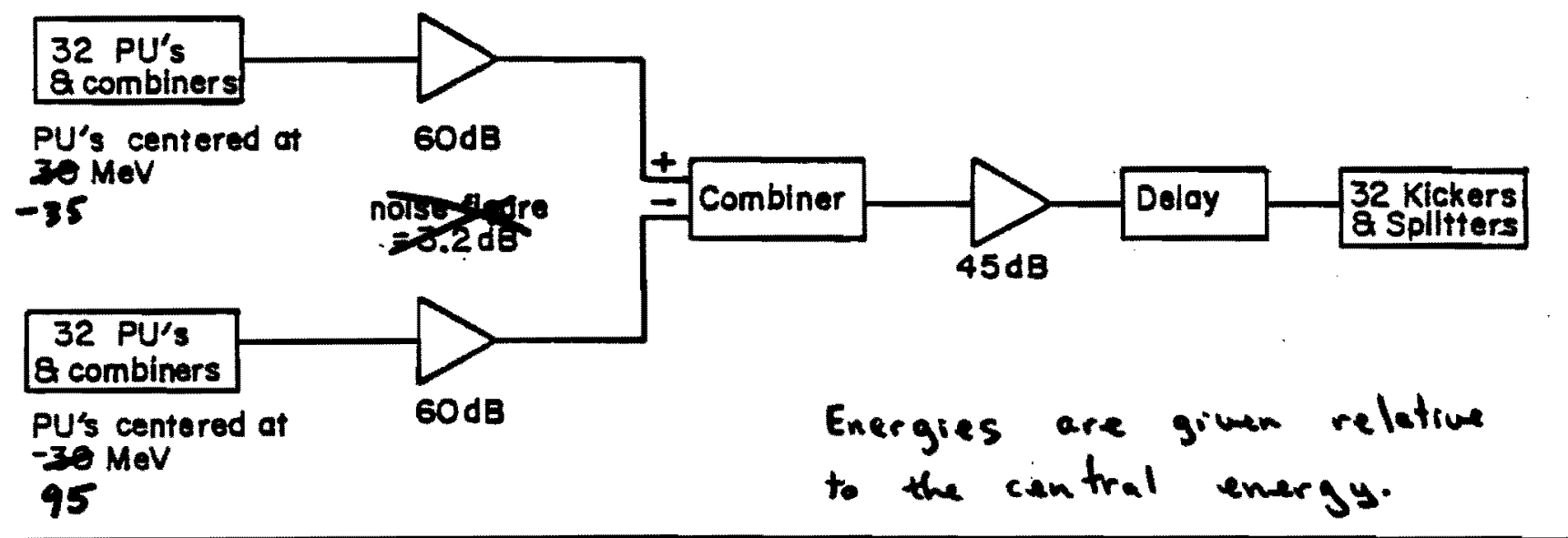

Fig. 5-5 

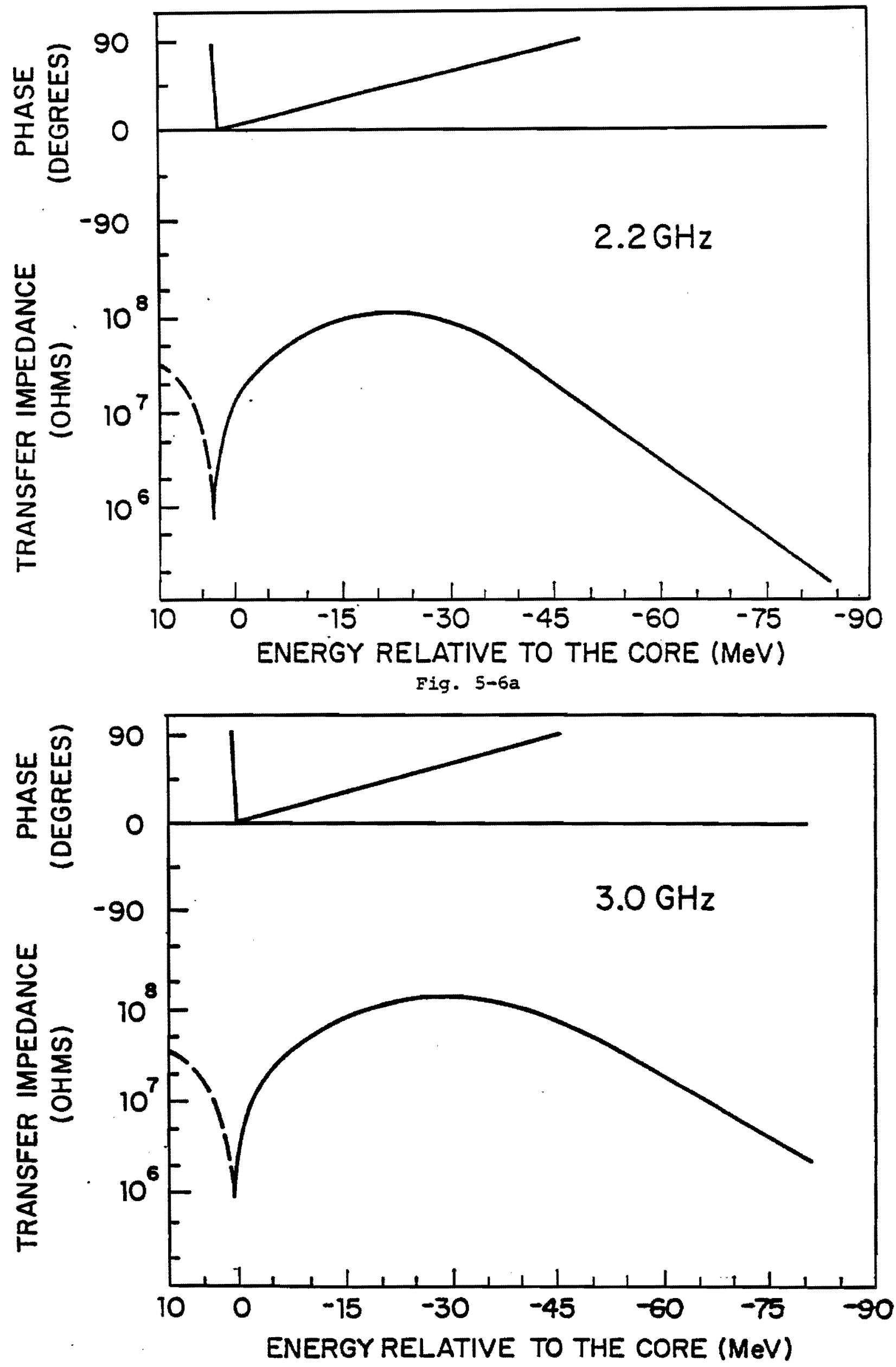

Fig. 5-6b 


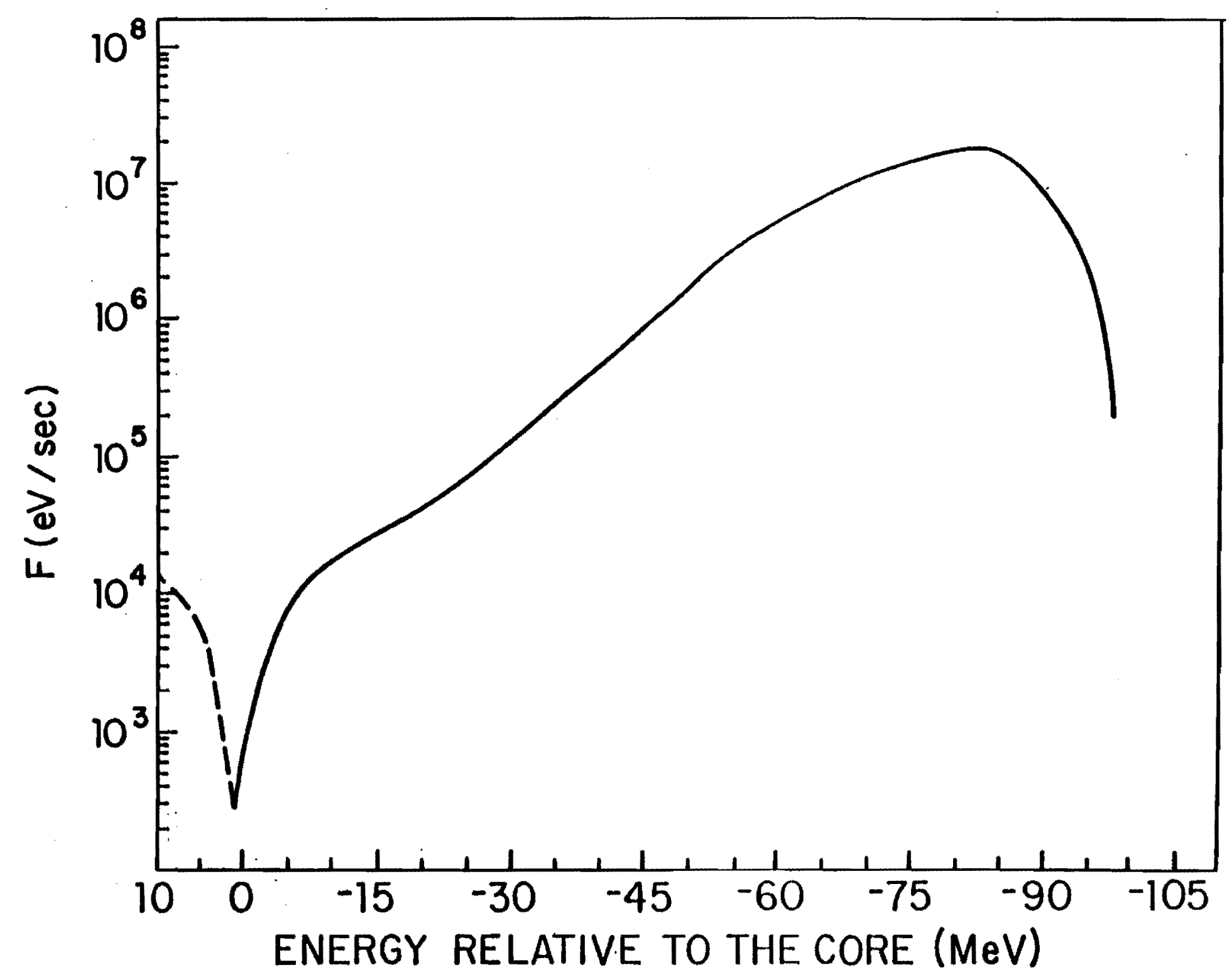

Fig. 5-8 

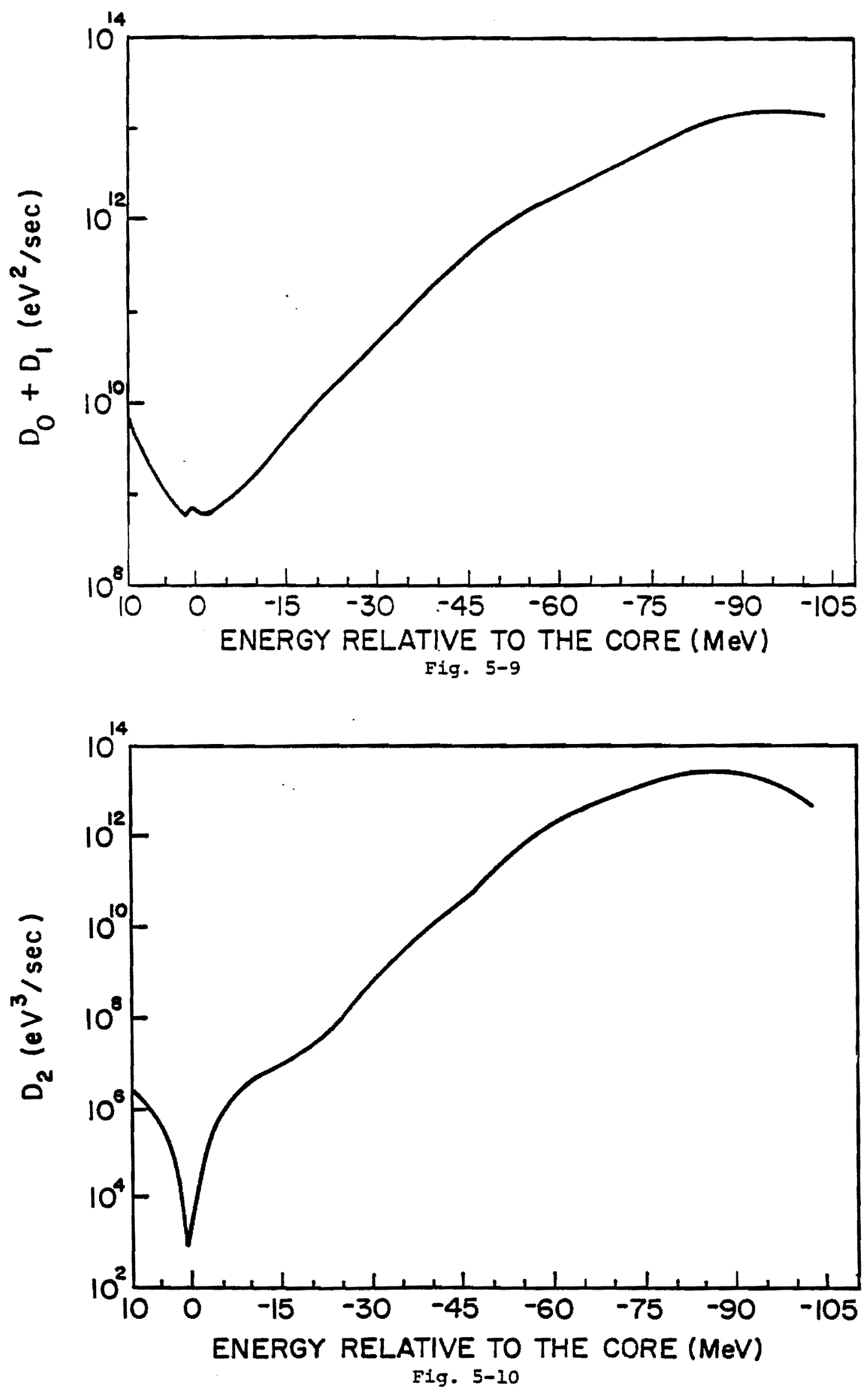


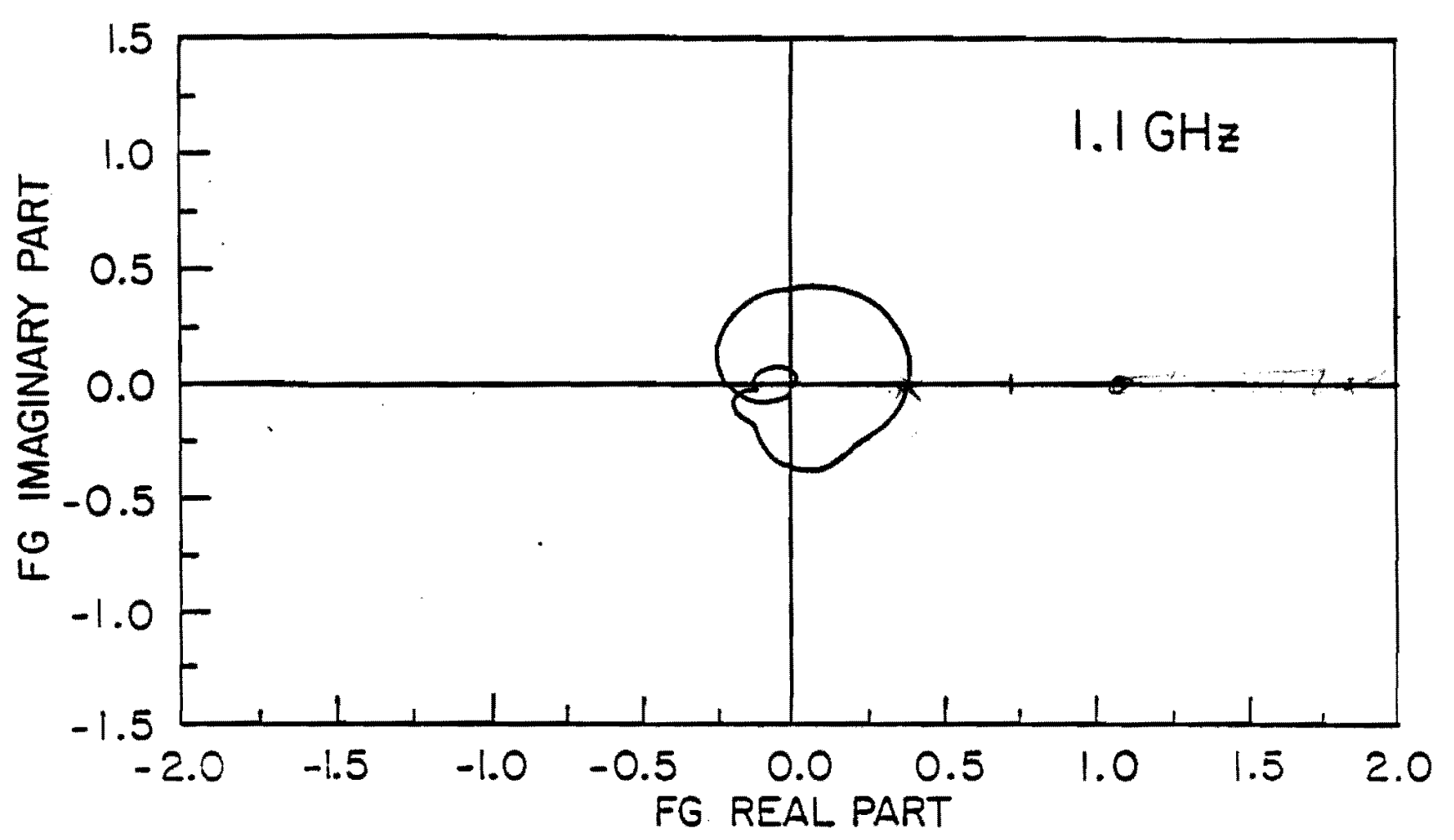

Eig. 5-11a

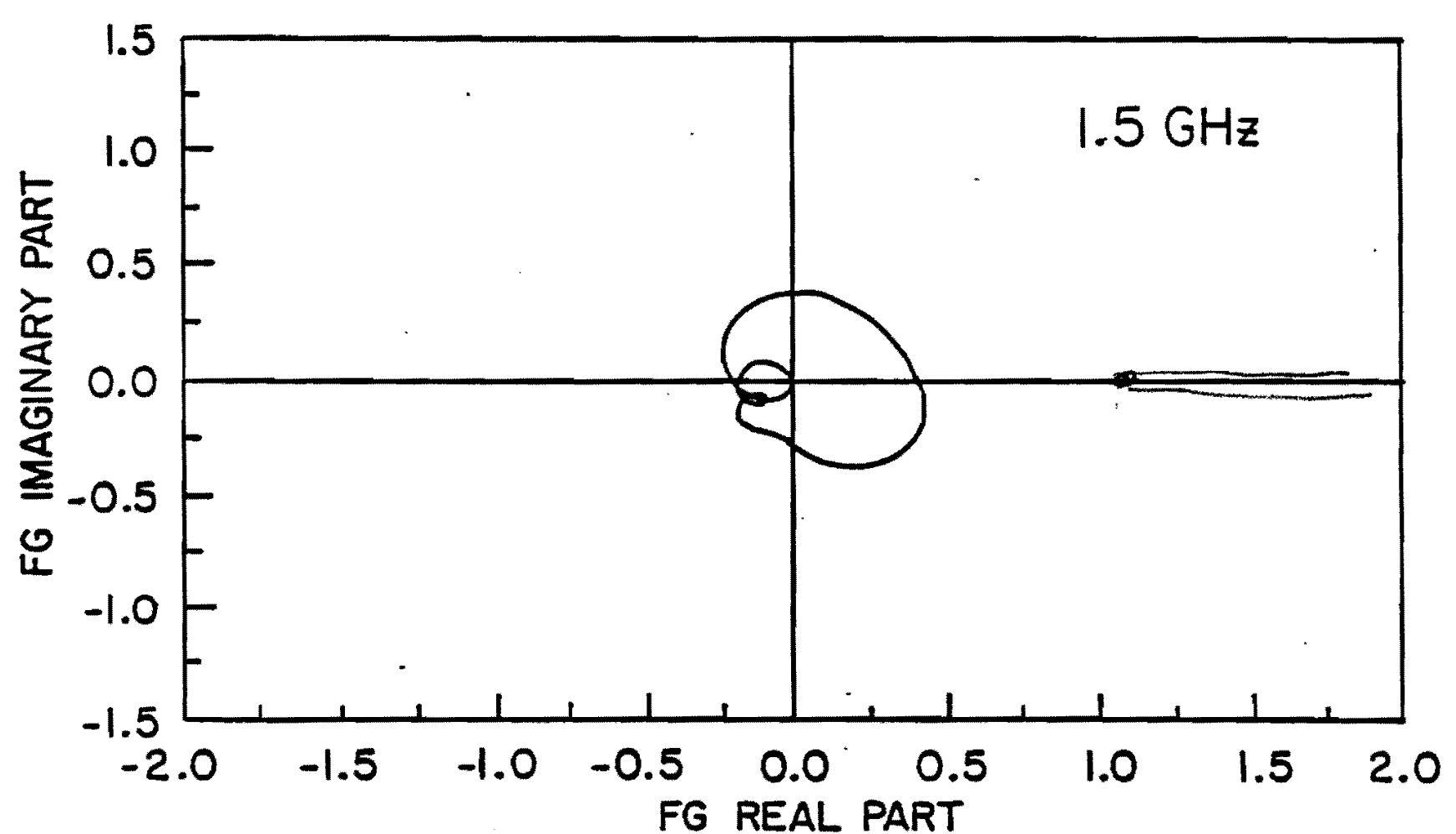

Fig. 5-11b 


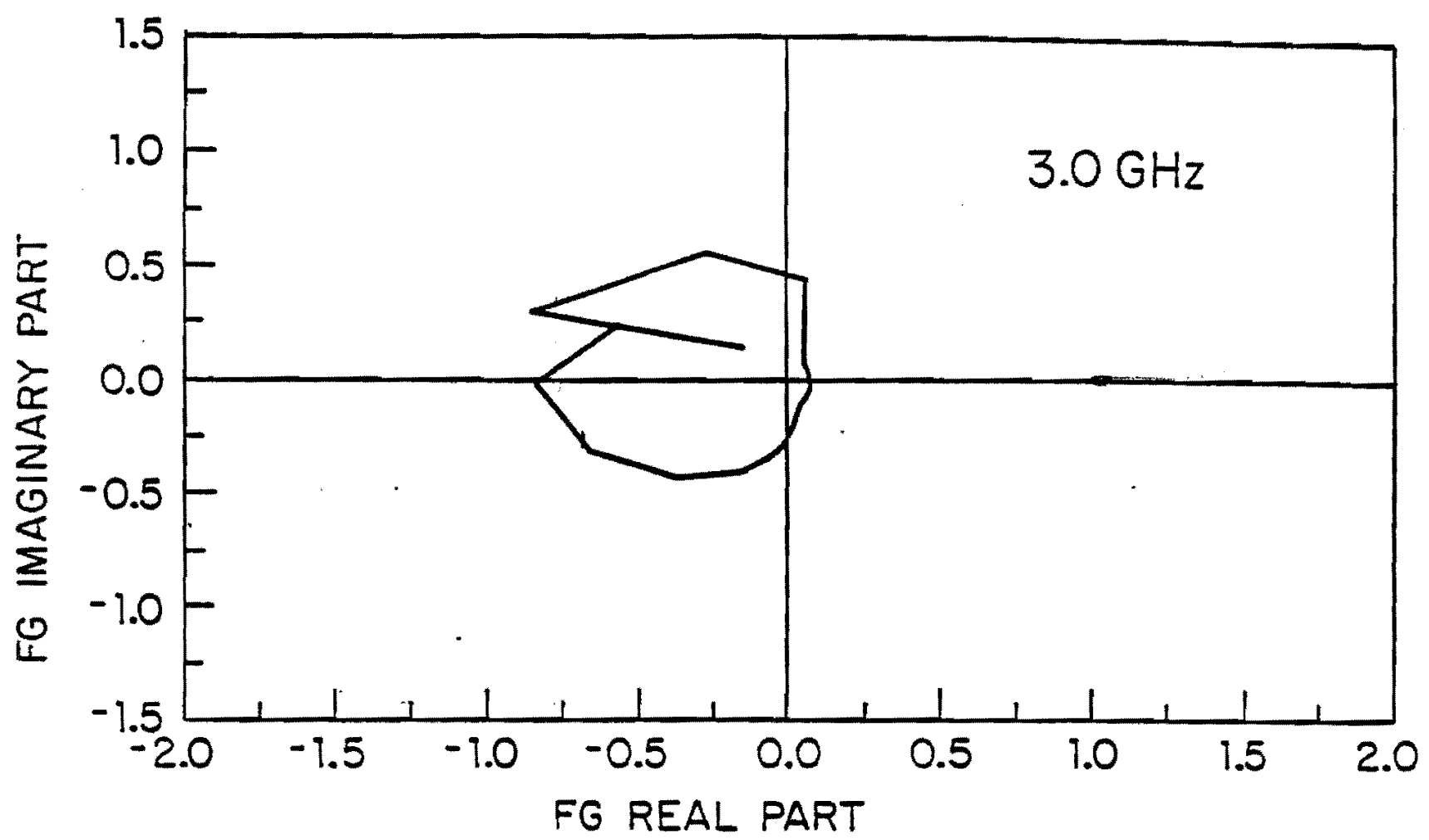

Fig. 5-I2b

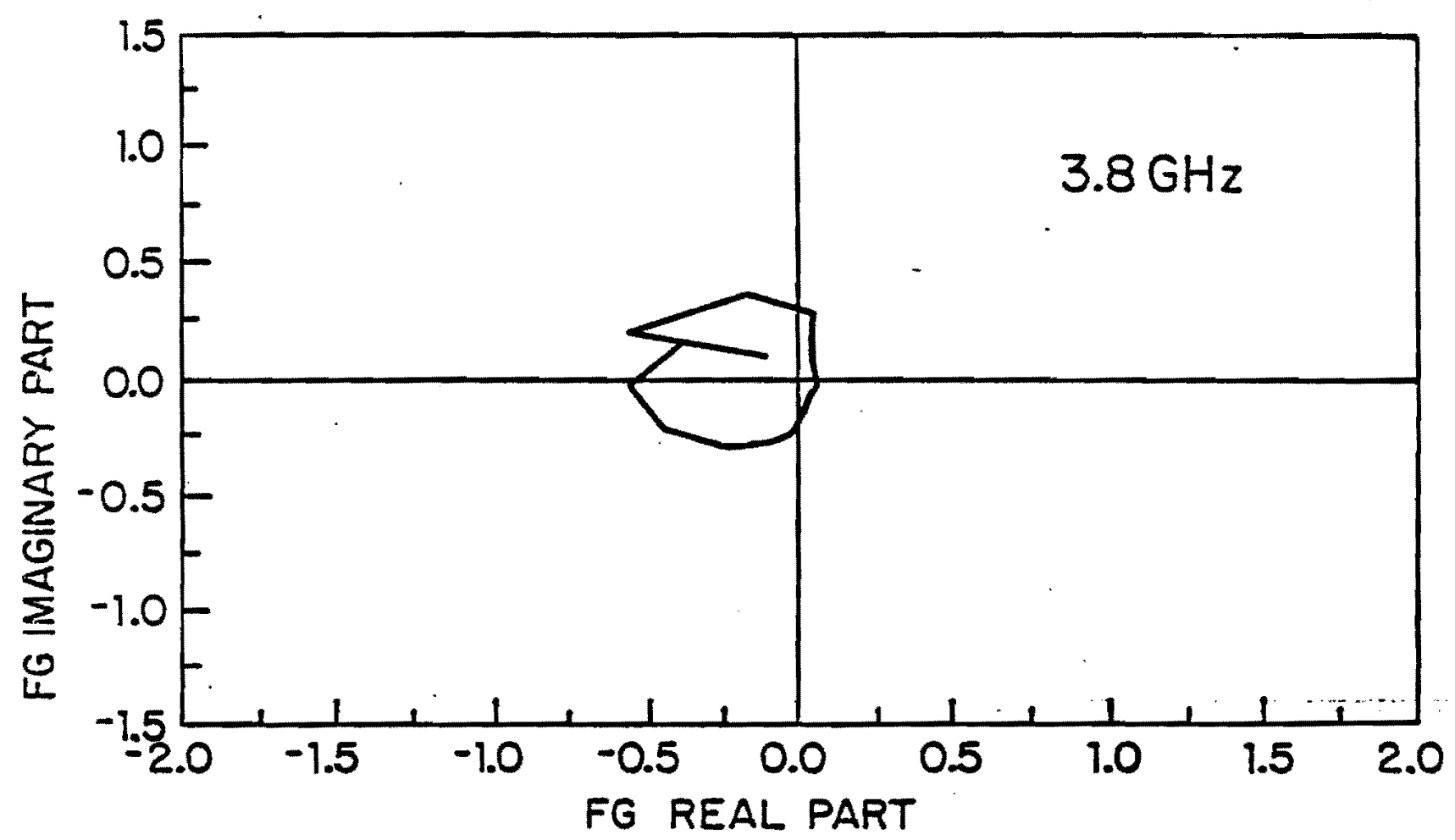

Fig. 5-12c 
CORE BETATRON COOLING SYSTEM 2-4 GHZ

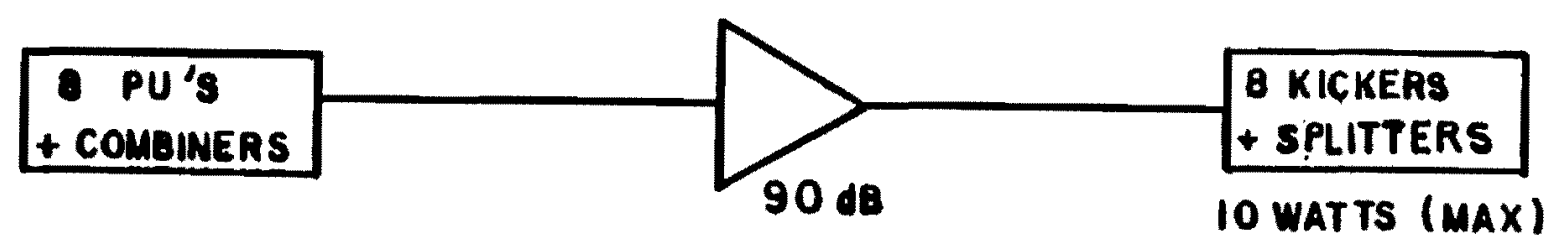

Fig. 5-13

STACK TAIL BETATRON COOLING 1-2 GHz

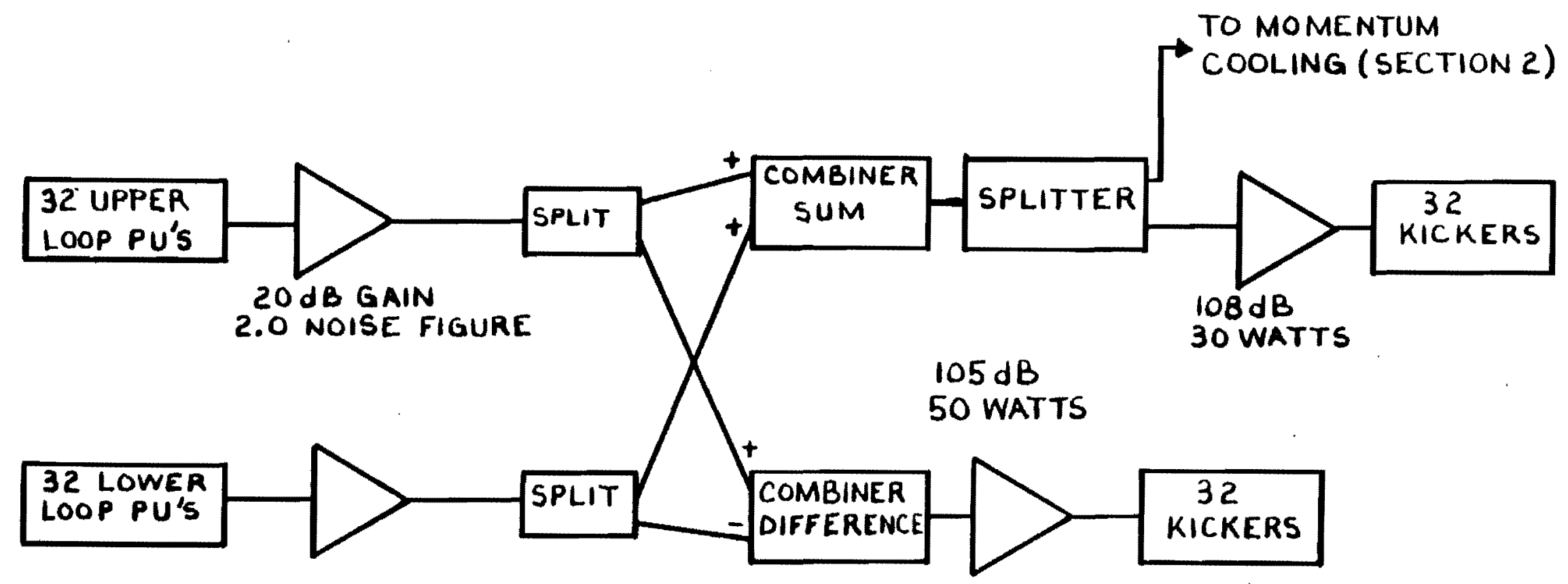

Fig. 5-14 

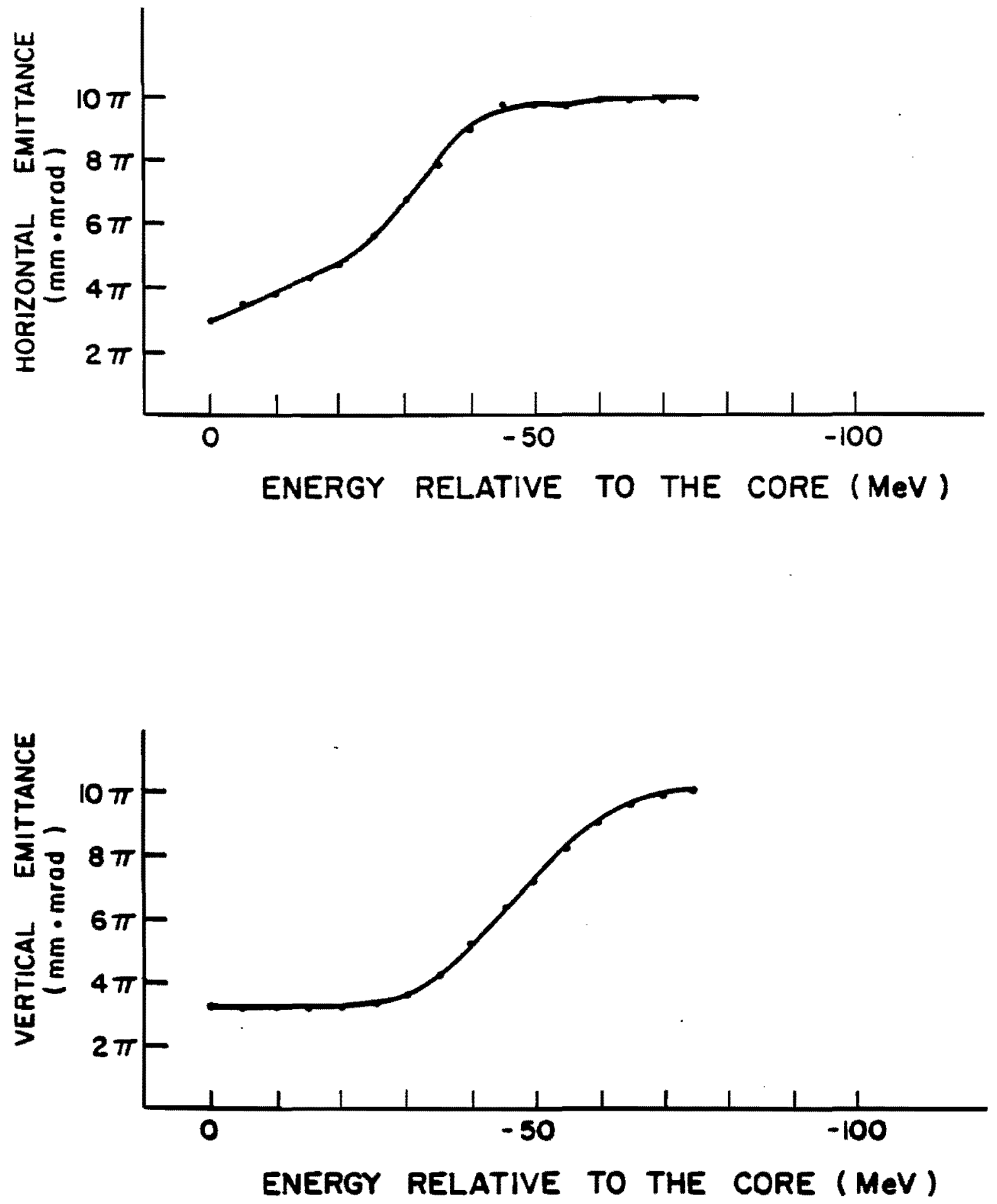

Fig. 5-15 


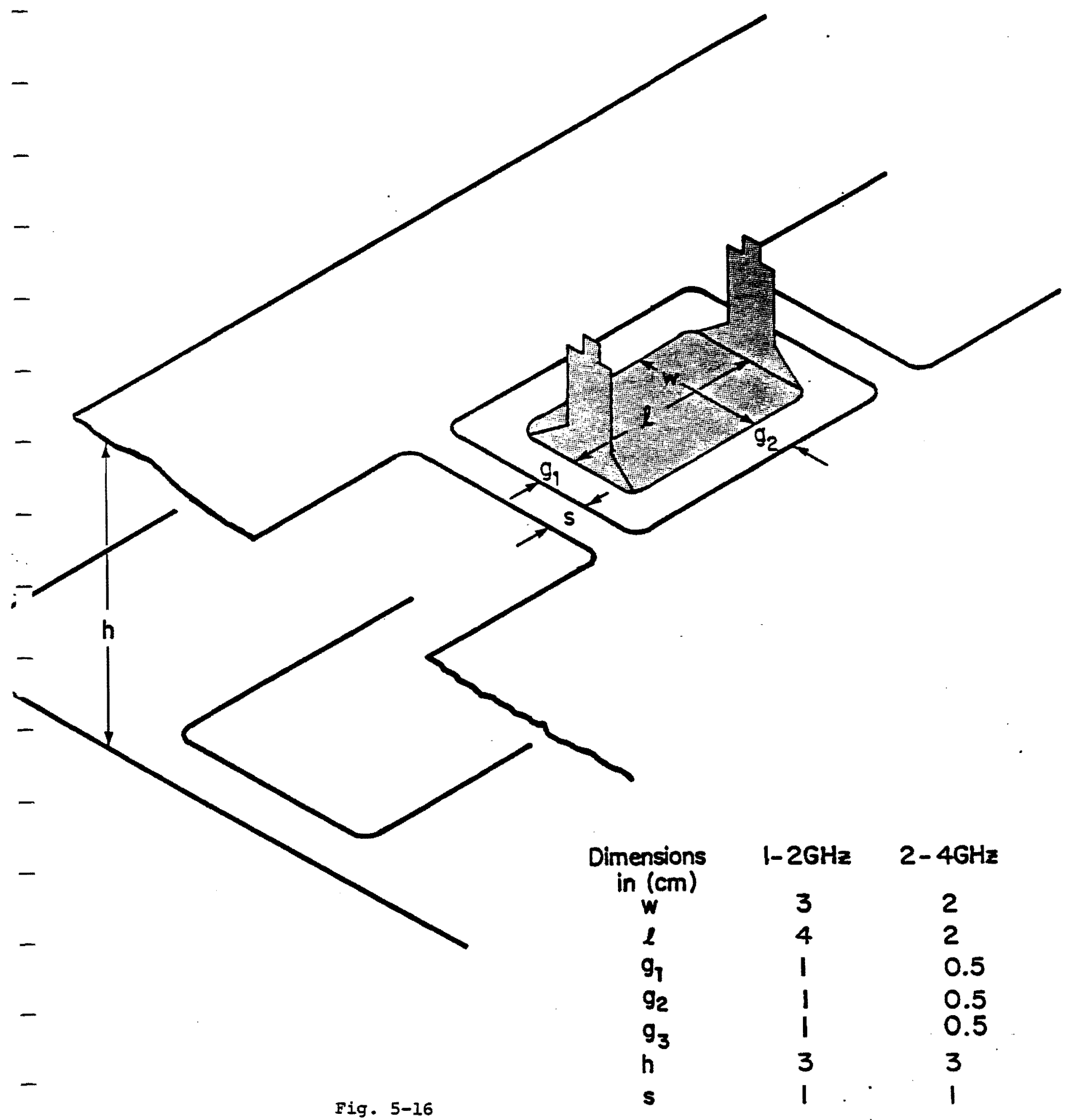

$g_{3}$ is the distance to the ground plane (not shown) above the pickup electrode 


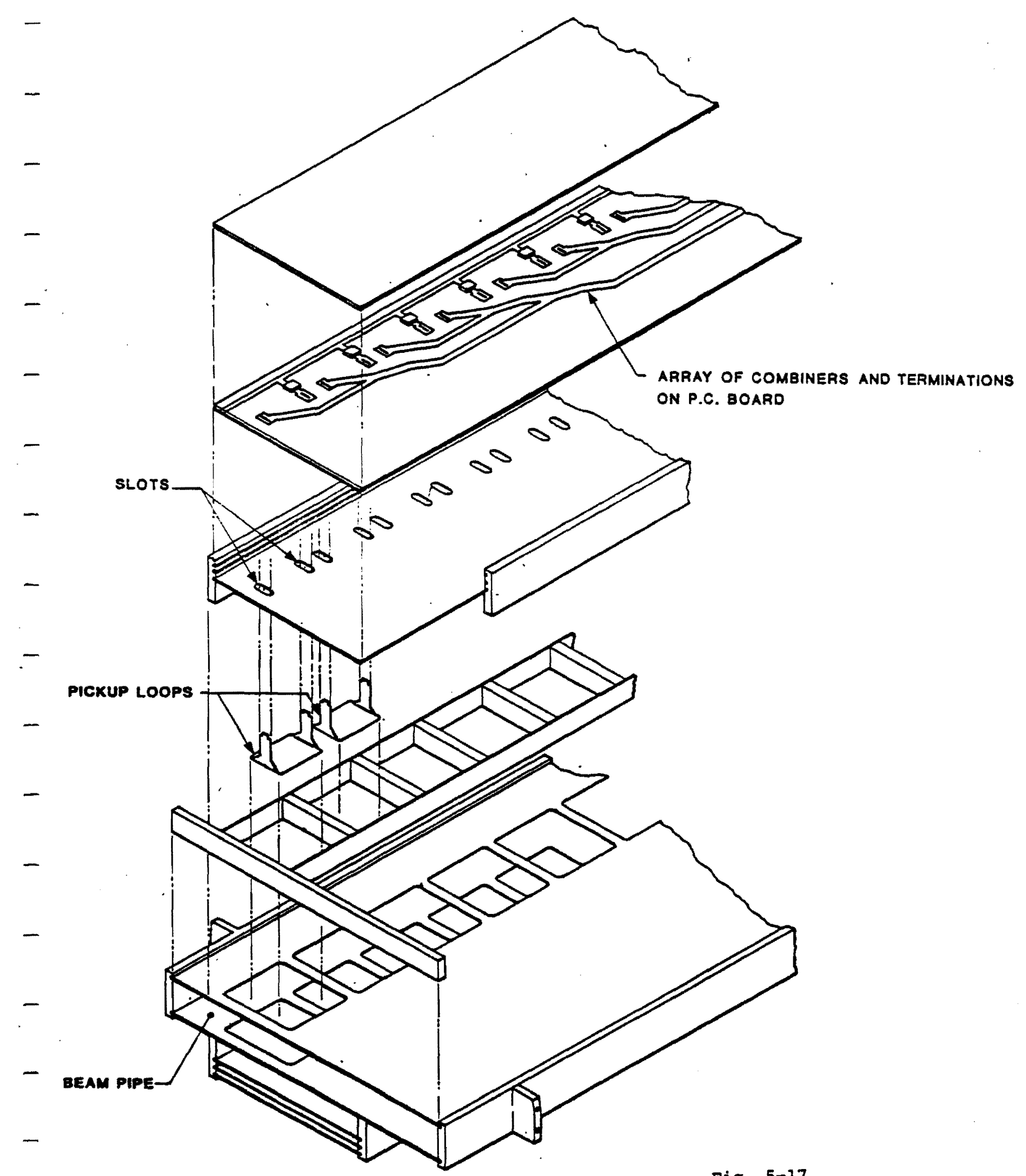

Fig. 5-17 


\begin{tabular}{|c|}
\hline 1 \\
\hline
\end{tabular}

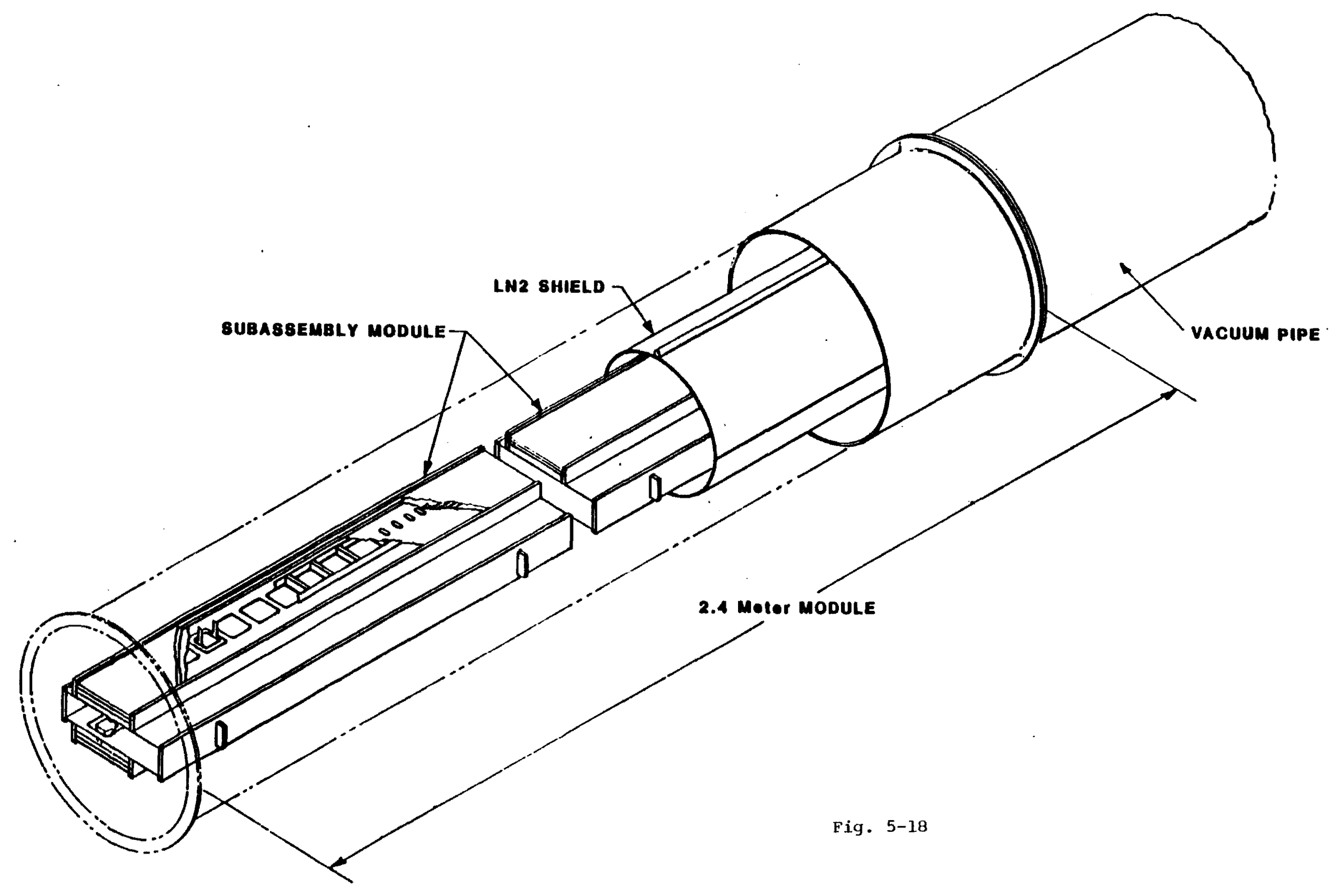




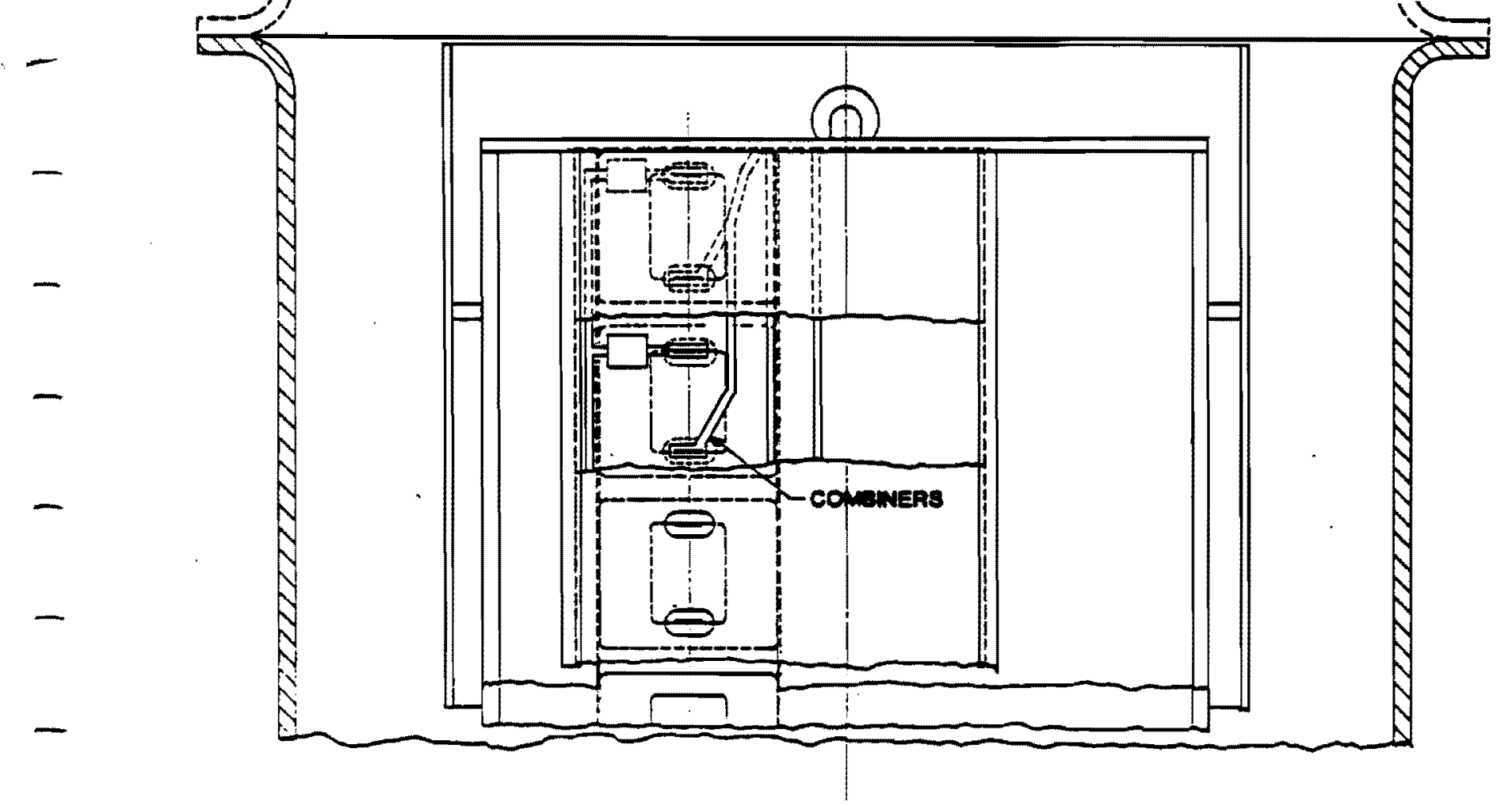




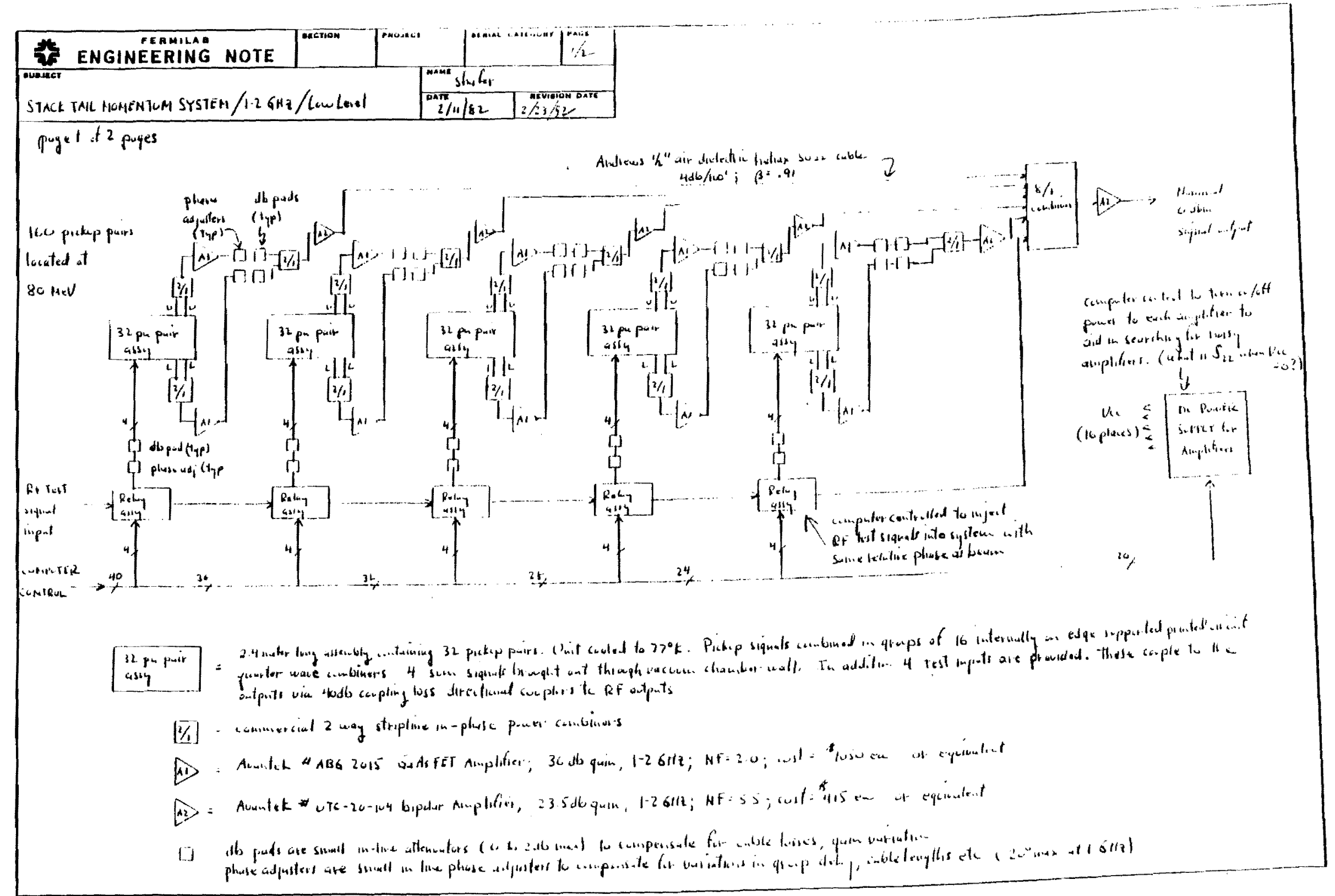

Fig. 5-20a 


(

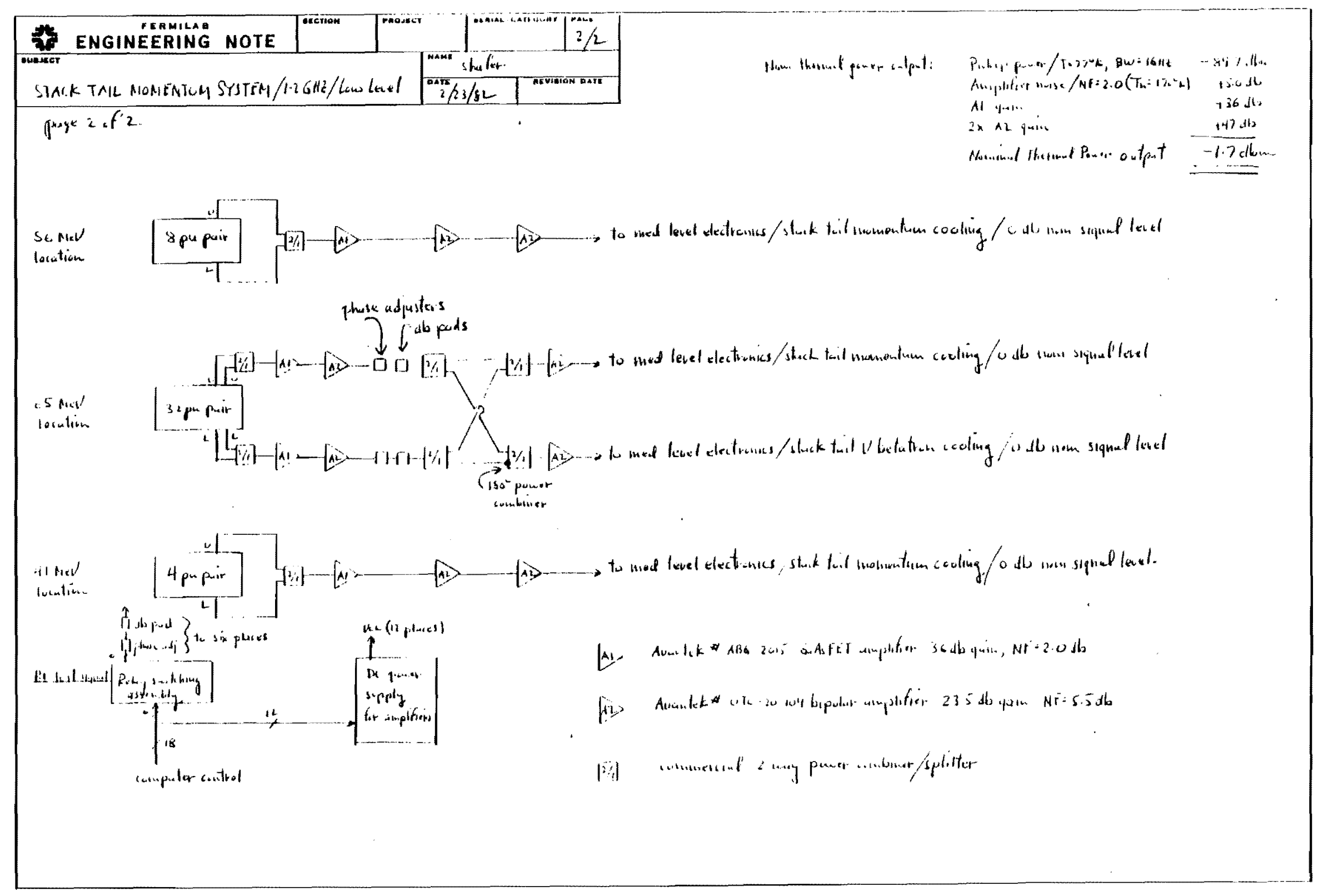

Fig. 5-20b 


1

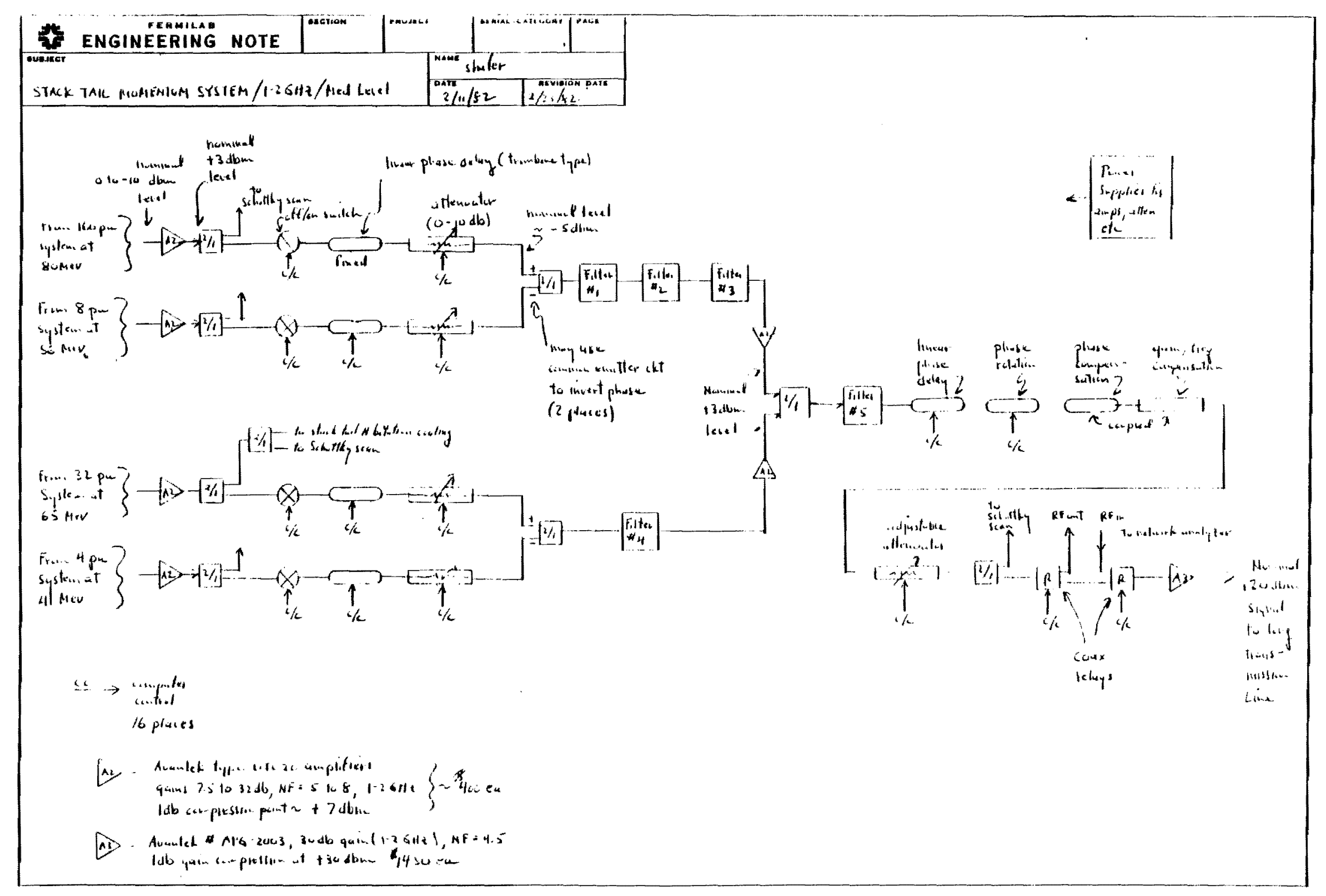

Fig. 5-21 
-2 GH: Power Stage<smiles>CCCCCCCCC</smiles>

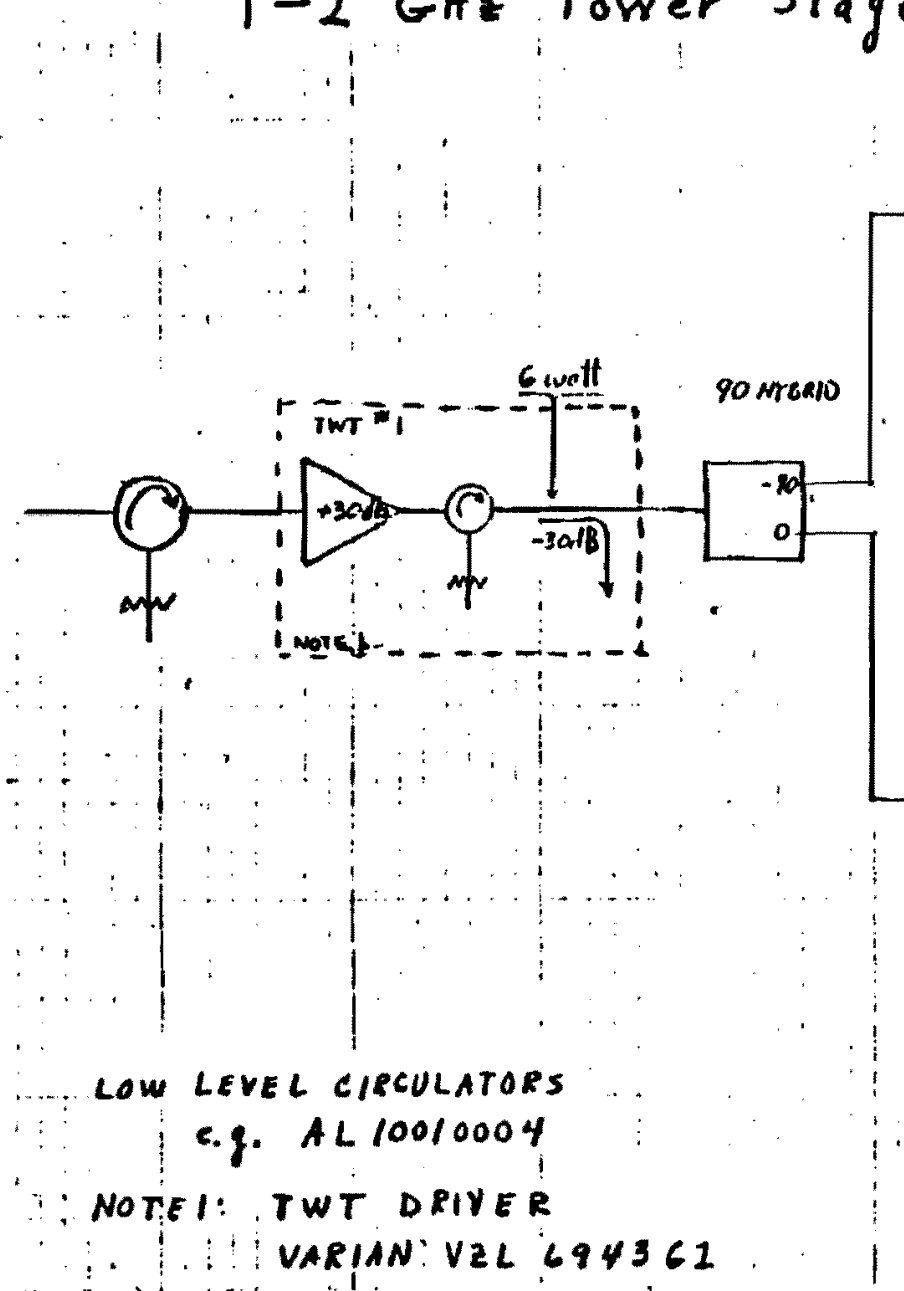

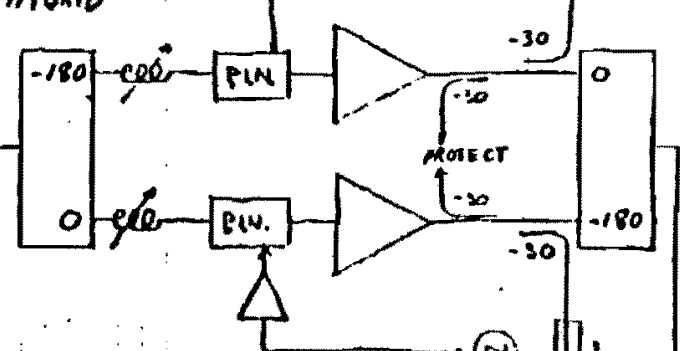<smiles>CCCCCCCCC</smiles> 



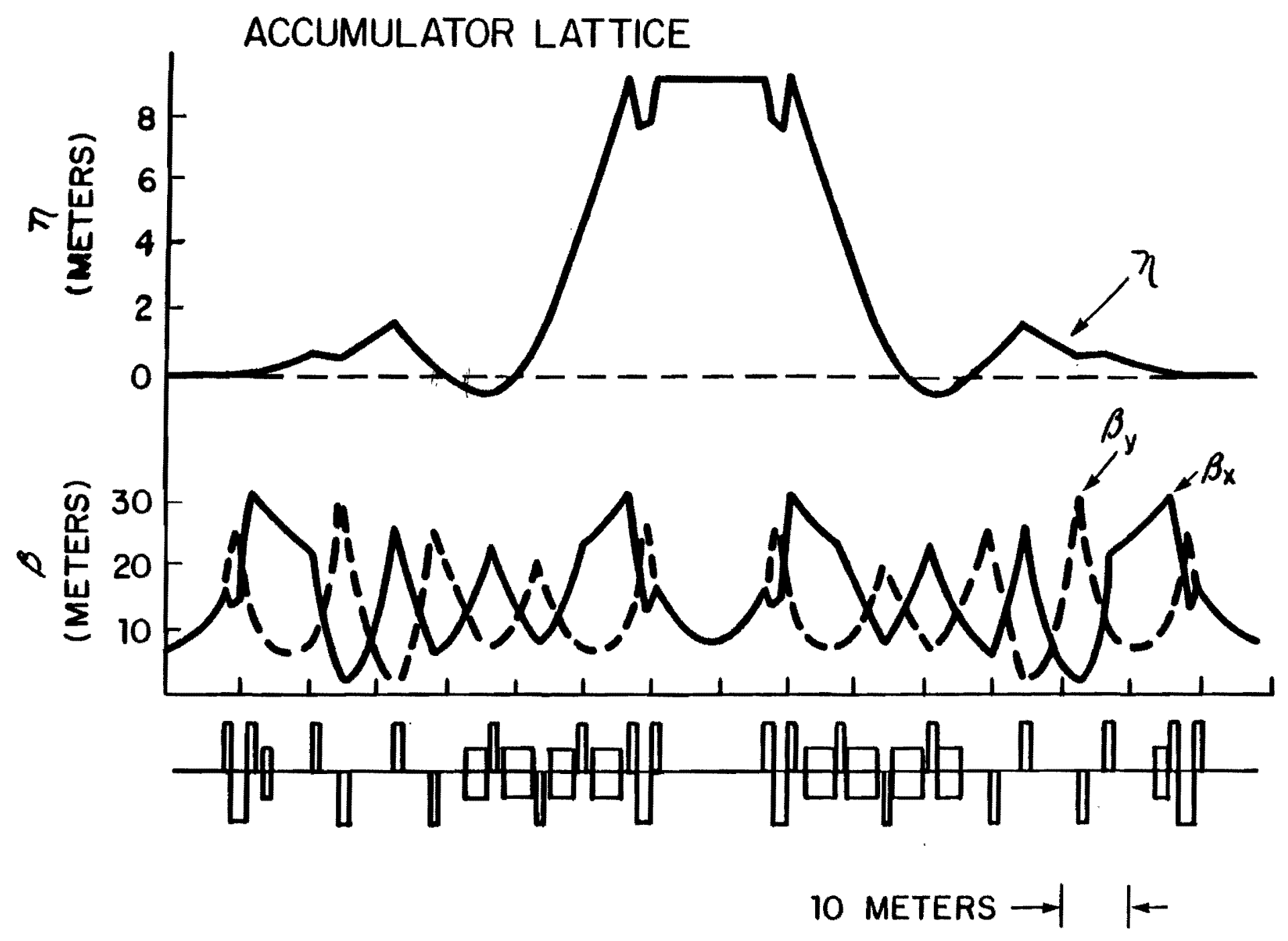

Fig. 5-24 
HORIZONTAL APERTURE REQUIREMENTS

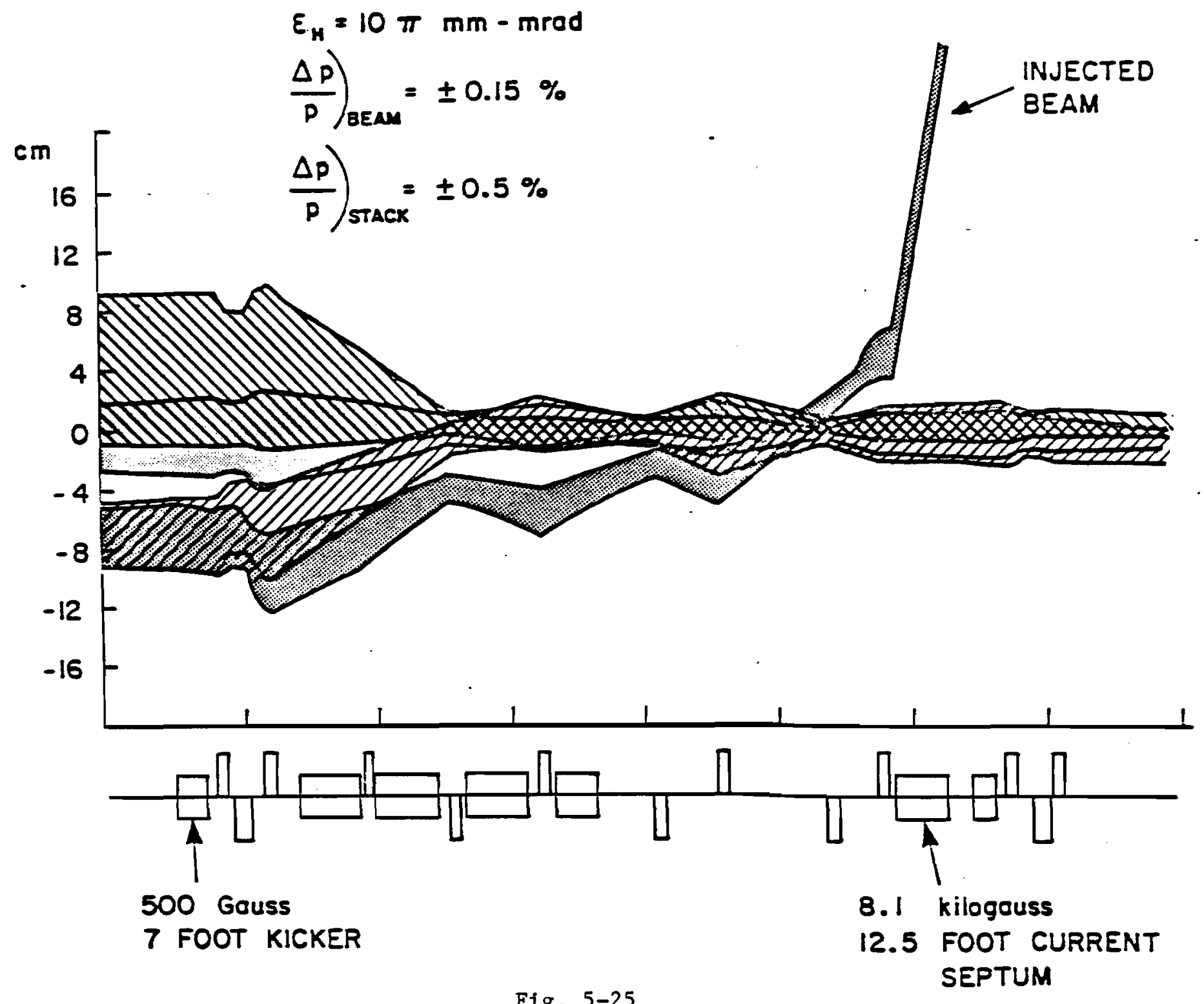




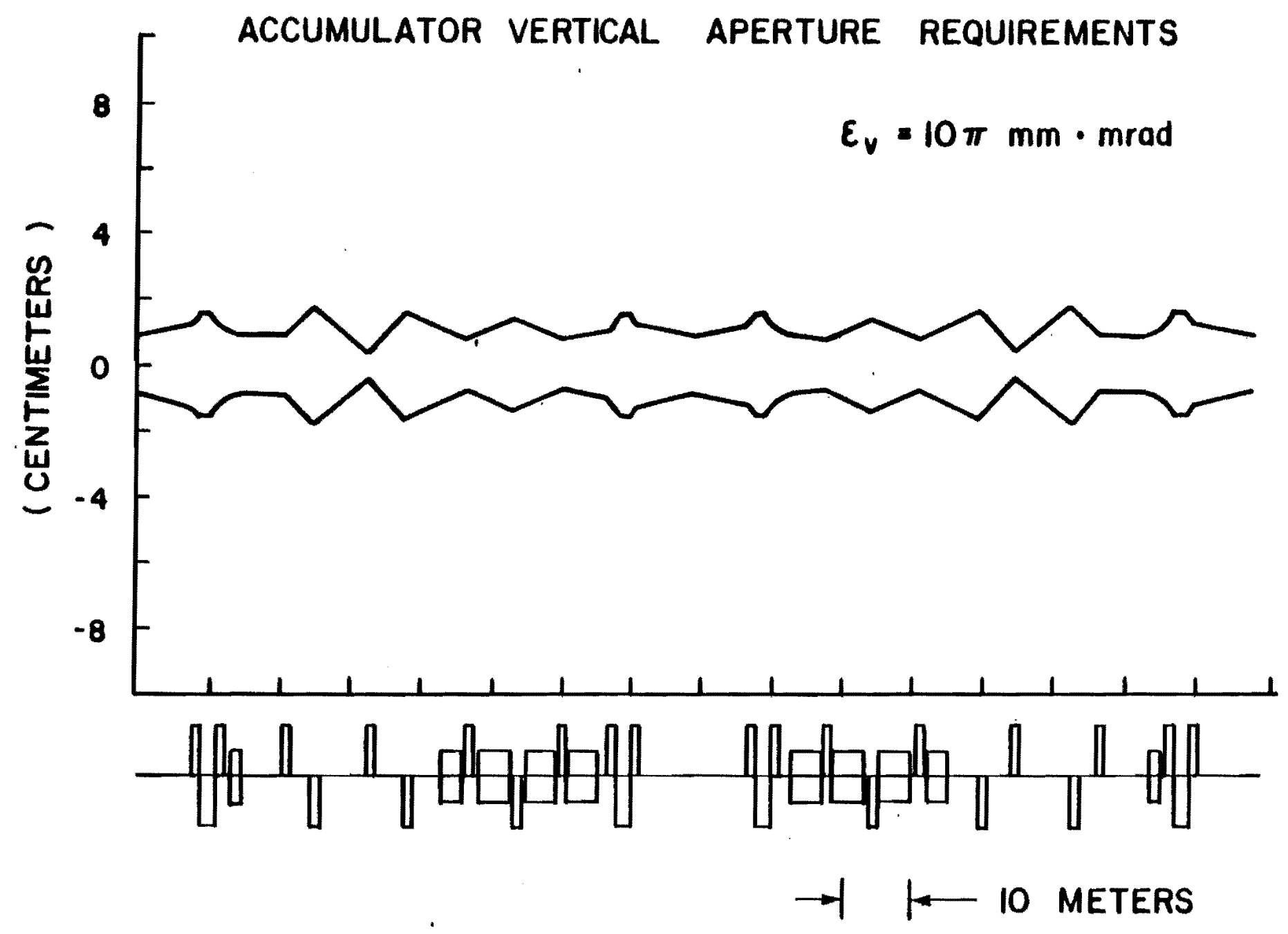

Fig. $5-26$ 


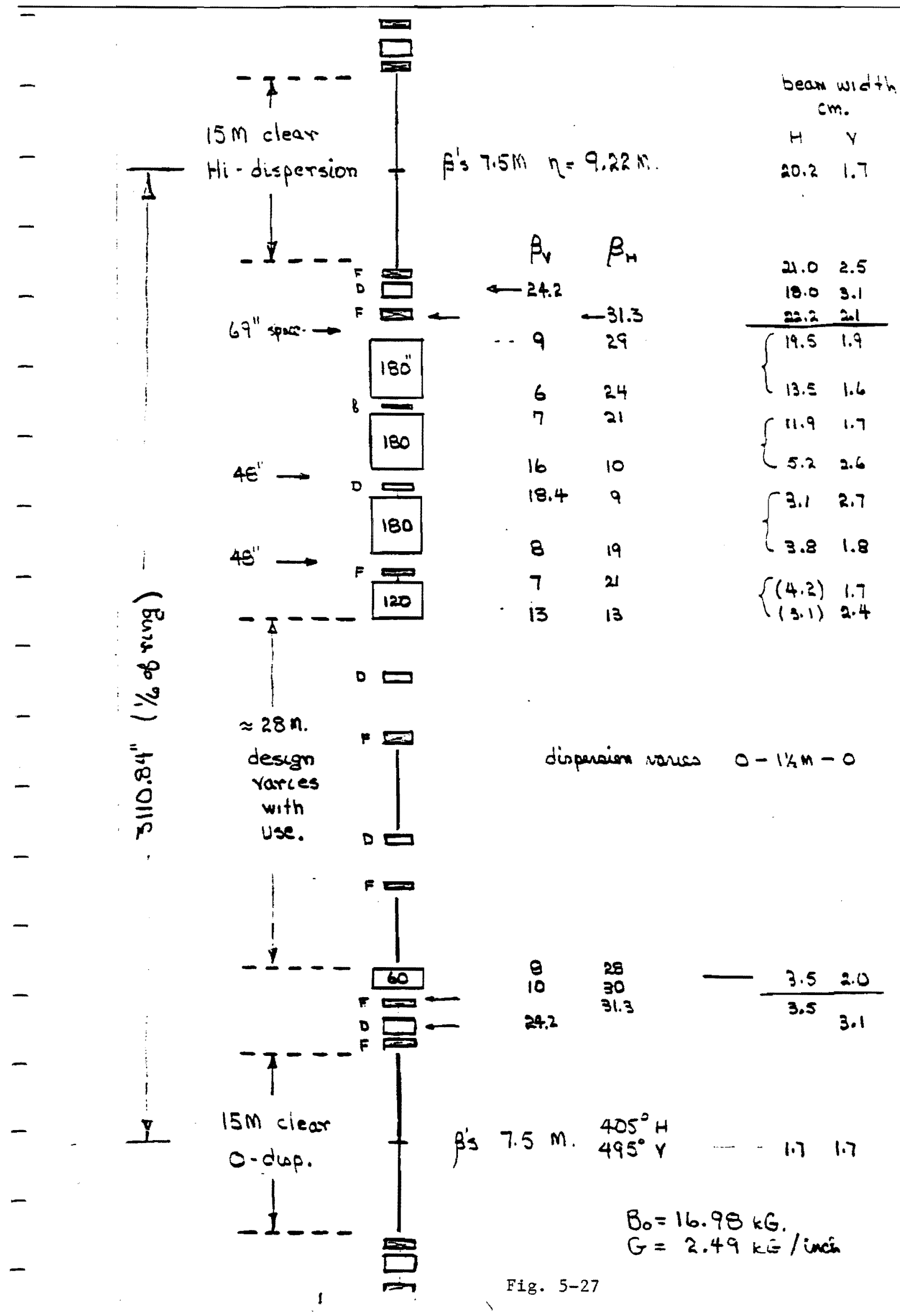




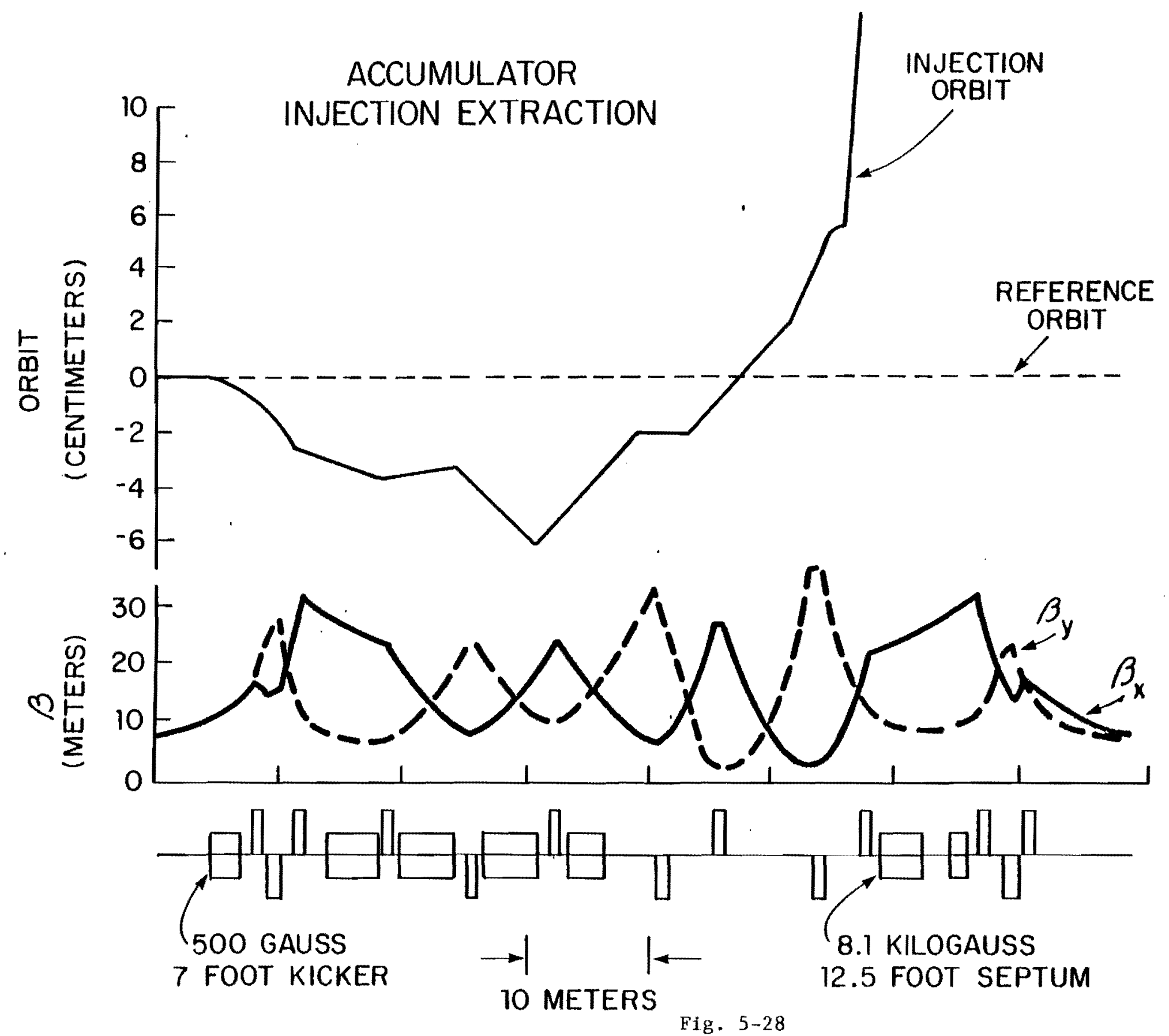




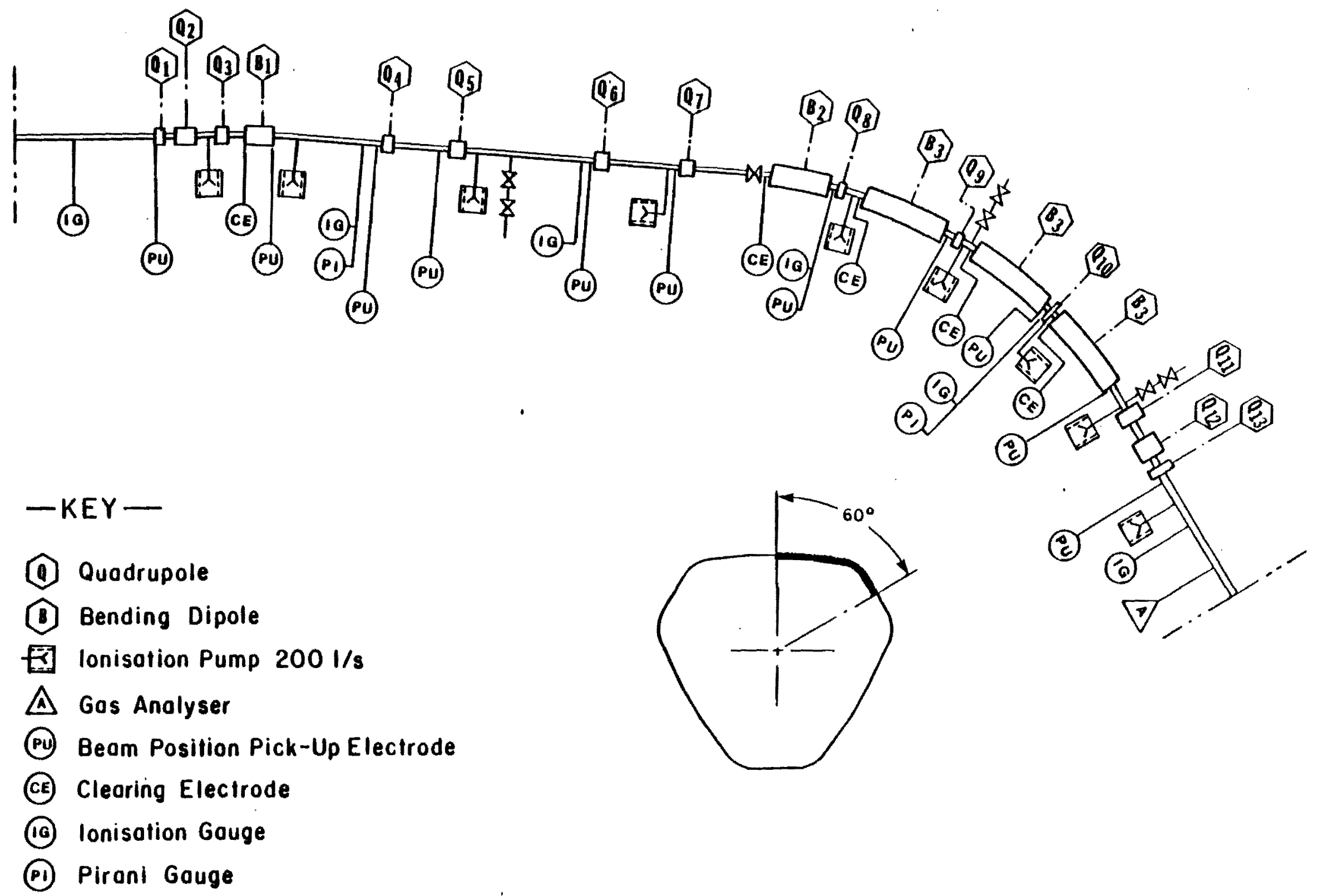

AD- Port for Mobile Pump Station 
Juint meI

25. 000

20. 000

15. 000

:0. 000
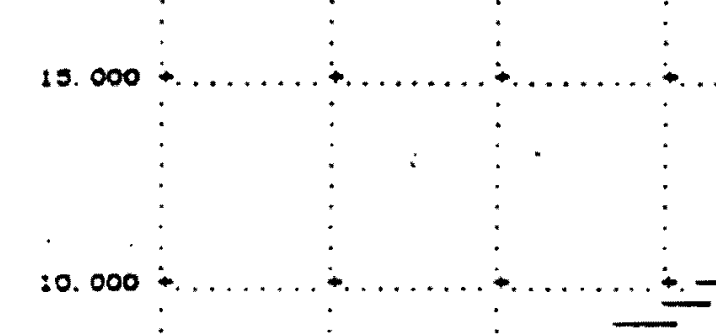

$\stackrel{9}{\underline{g}}$

$\equiv$

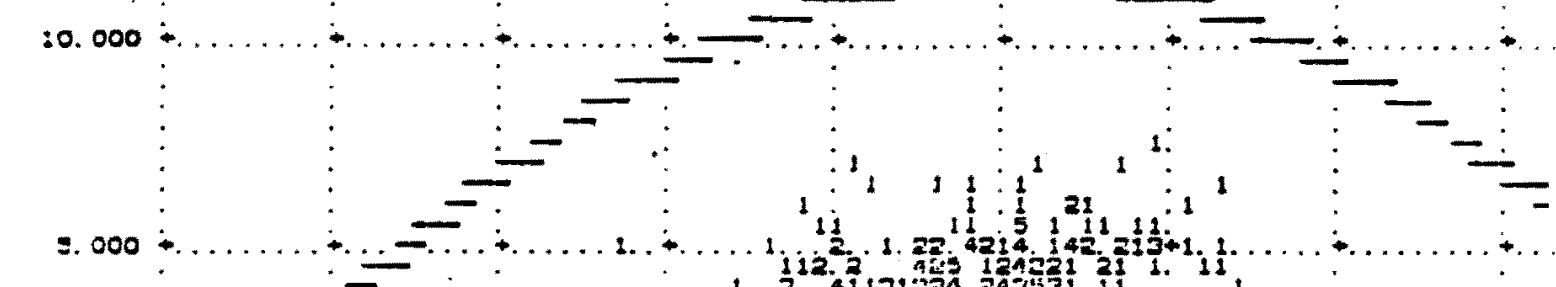

$\underline{\underline{g}}$

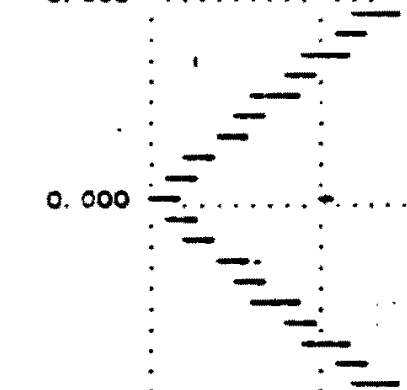

$-15,000$

B.-Gansoian $\pm \frac{\pi}{3} \quad k=53$

\section{1,2 el see.}

$-20.000$

$-25.000$

$-3.142$

$-2313$

$-1.805$

$-1.237$

$-.5$

$-.000$

.628

1. 257

1. 884,

2313

1. 142

PMI (RF PHASES AAE PHI AND (HQ/HI) IOPK)

Fig. 6-1 


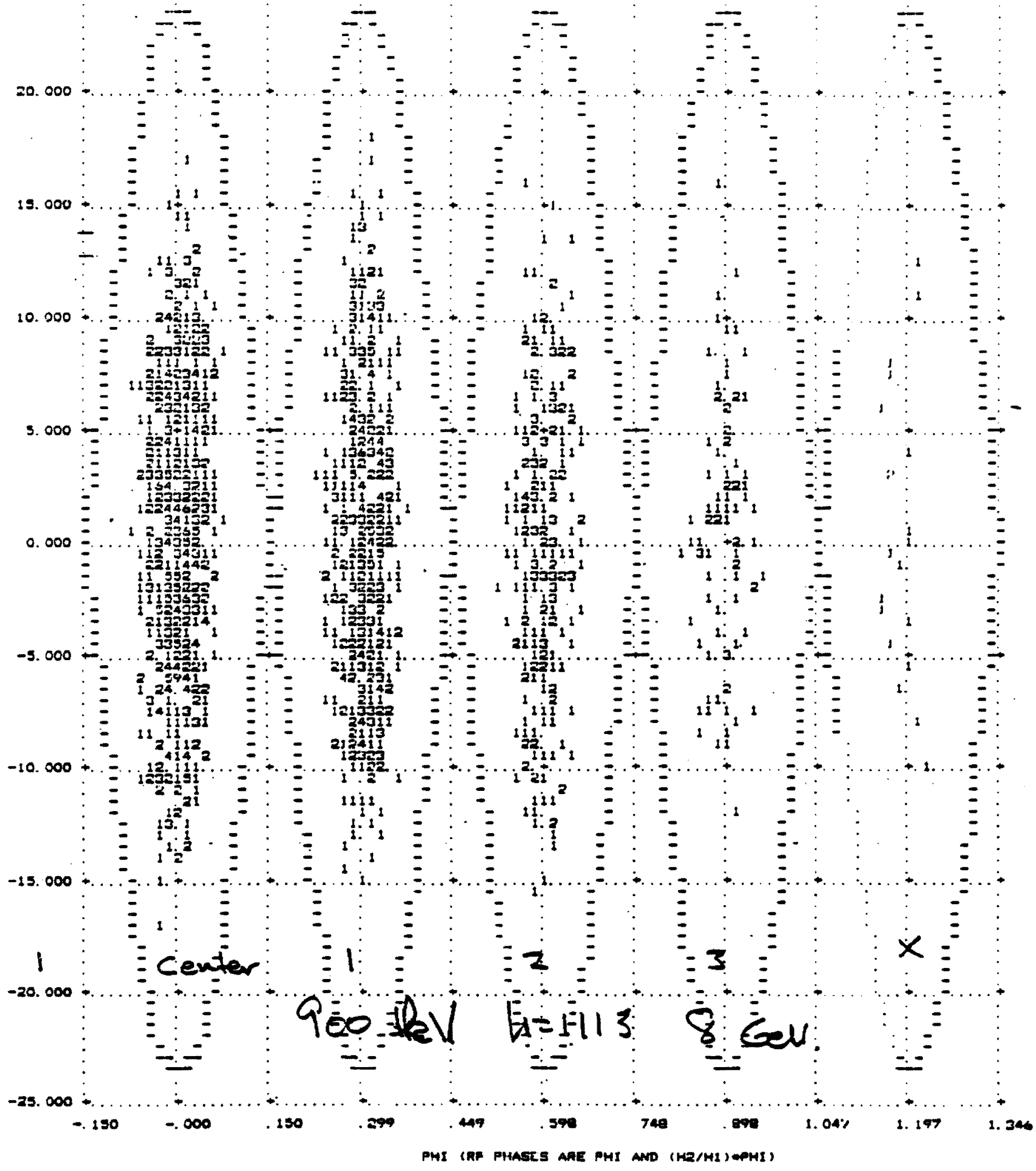

Fig. 6-3 


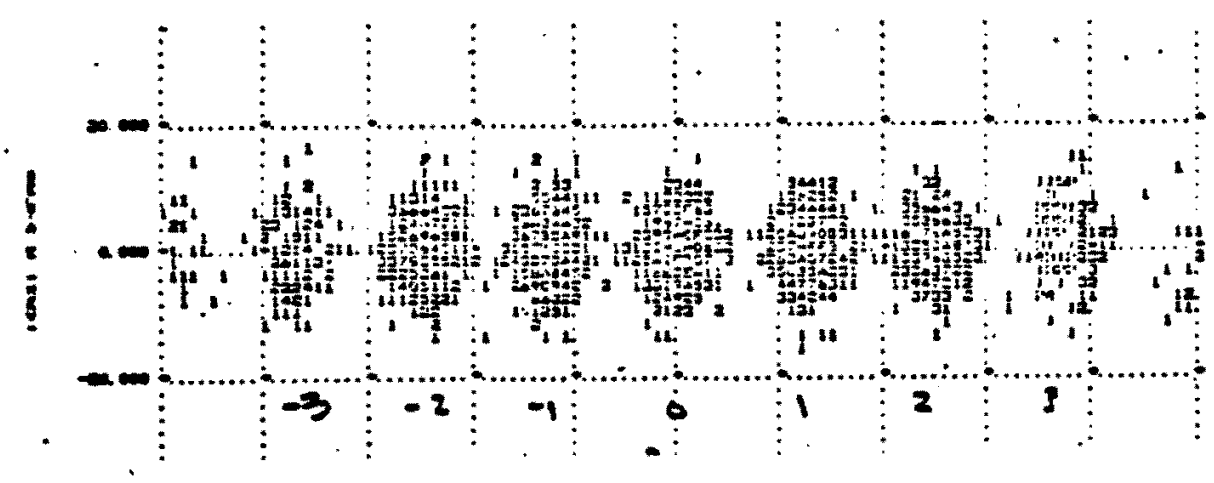

(a)
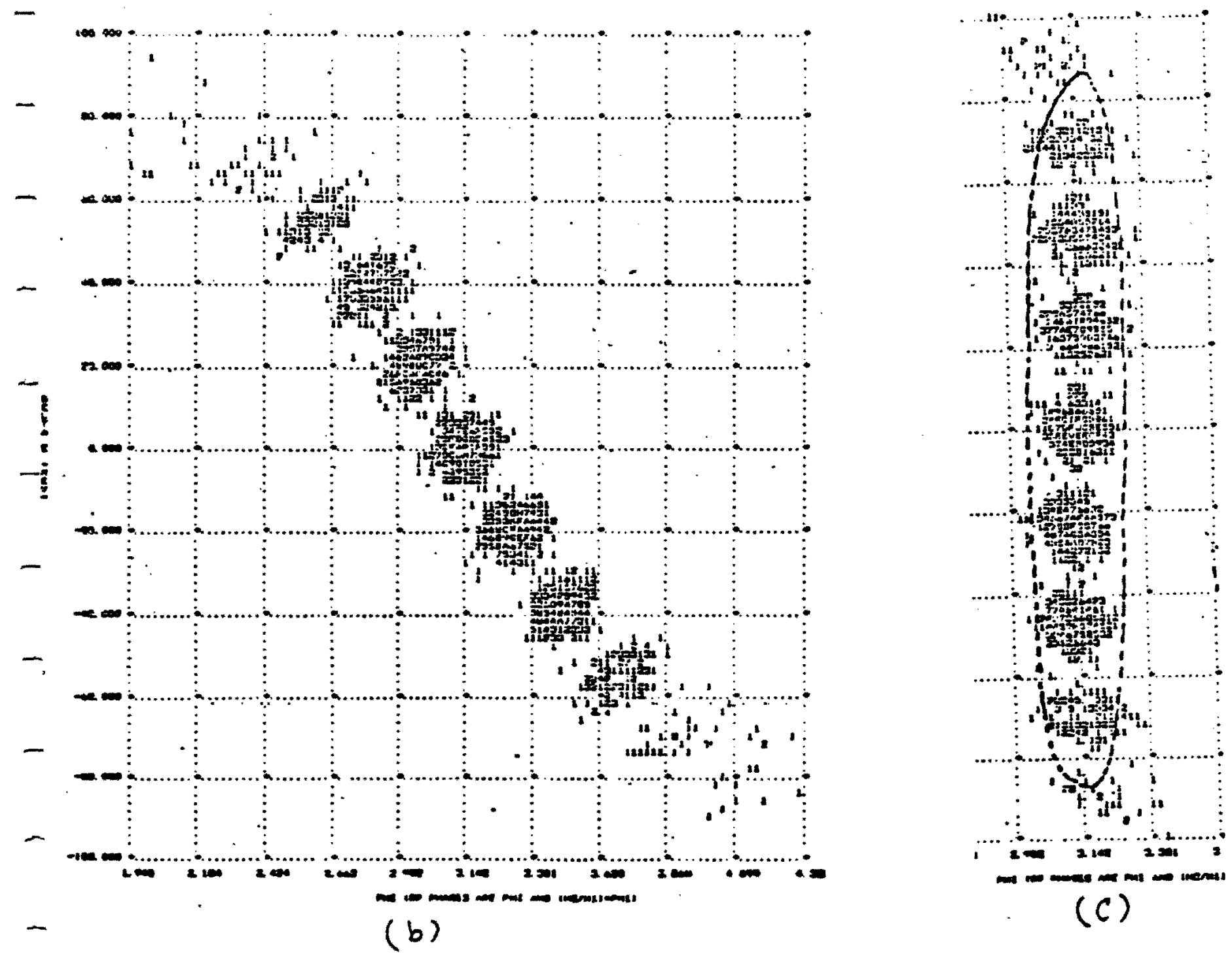

Fig. 6-4 


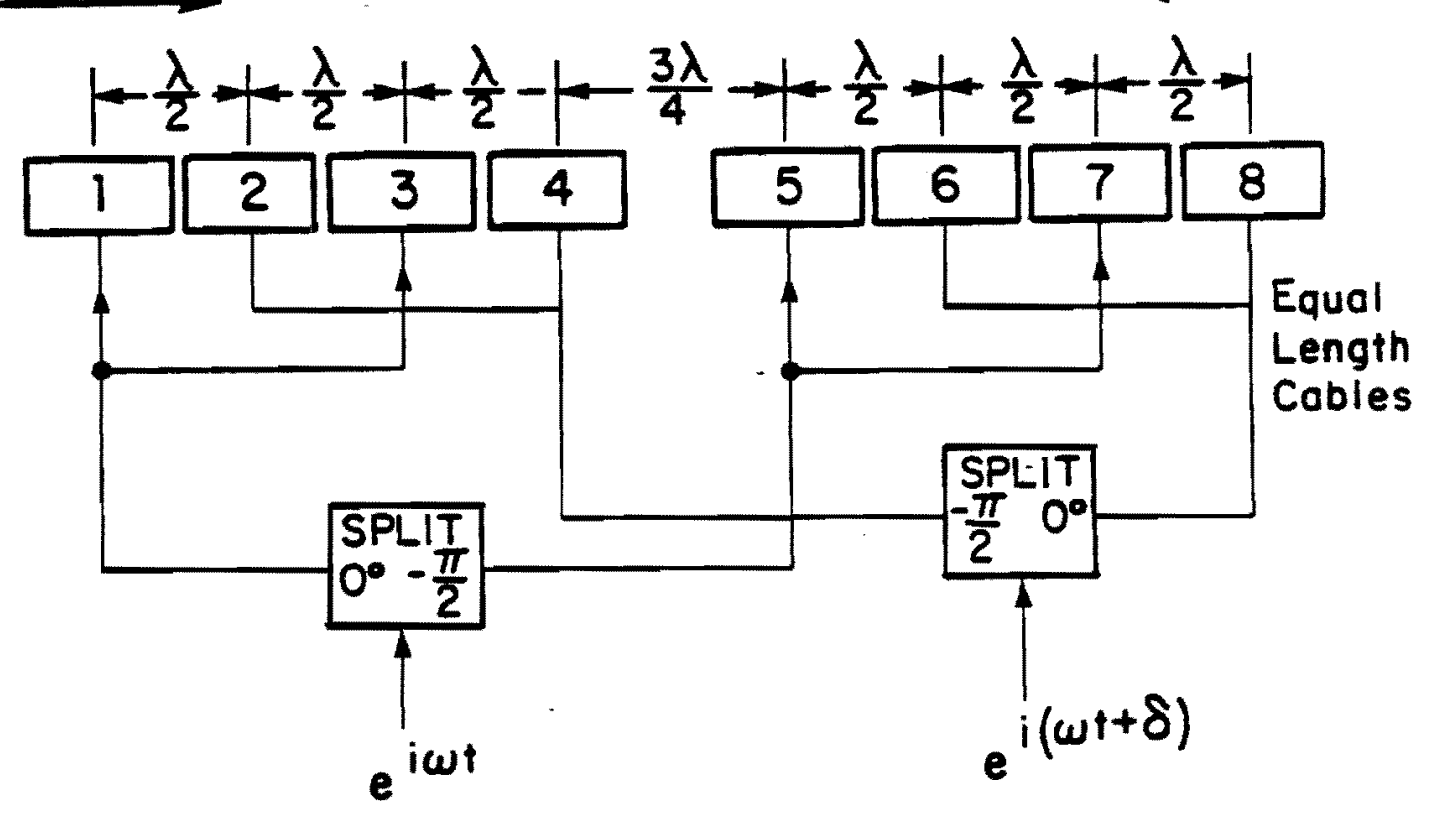

(a)

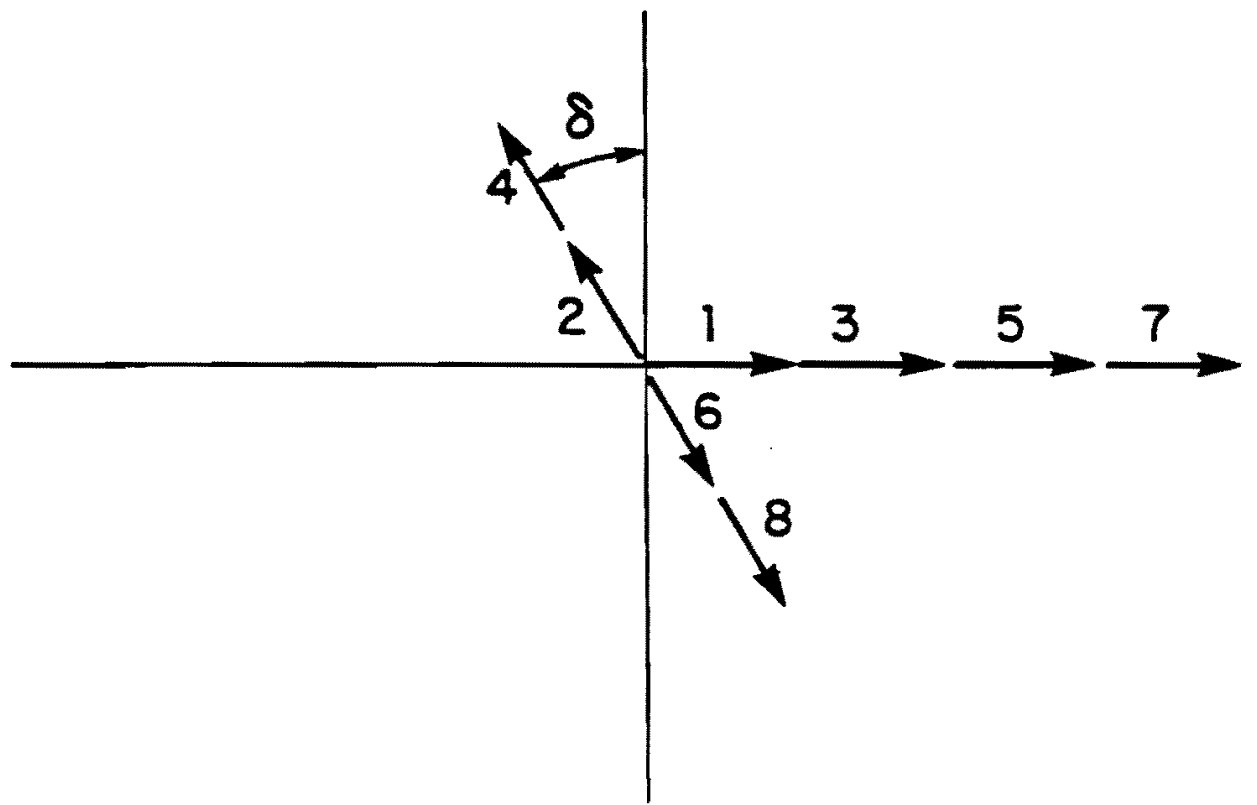

(b)

Fig. 6-6 


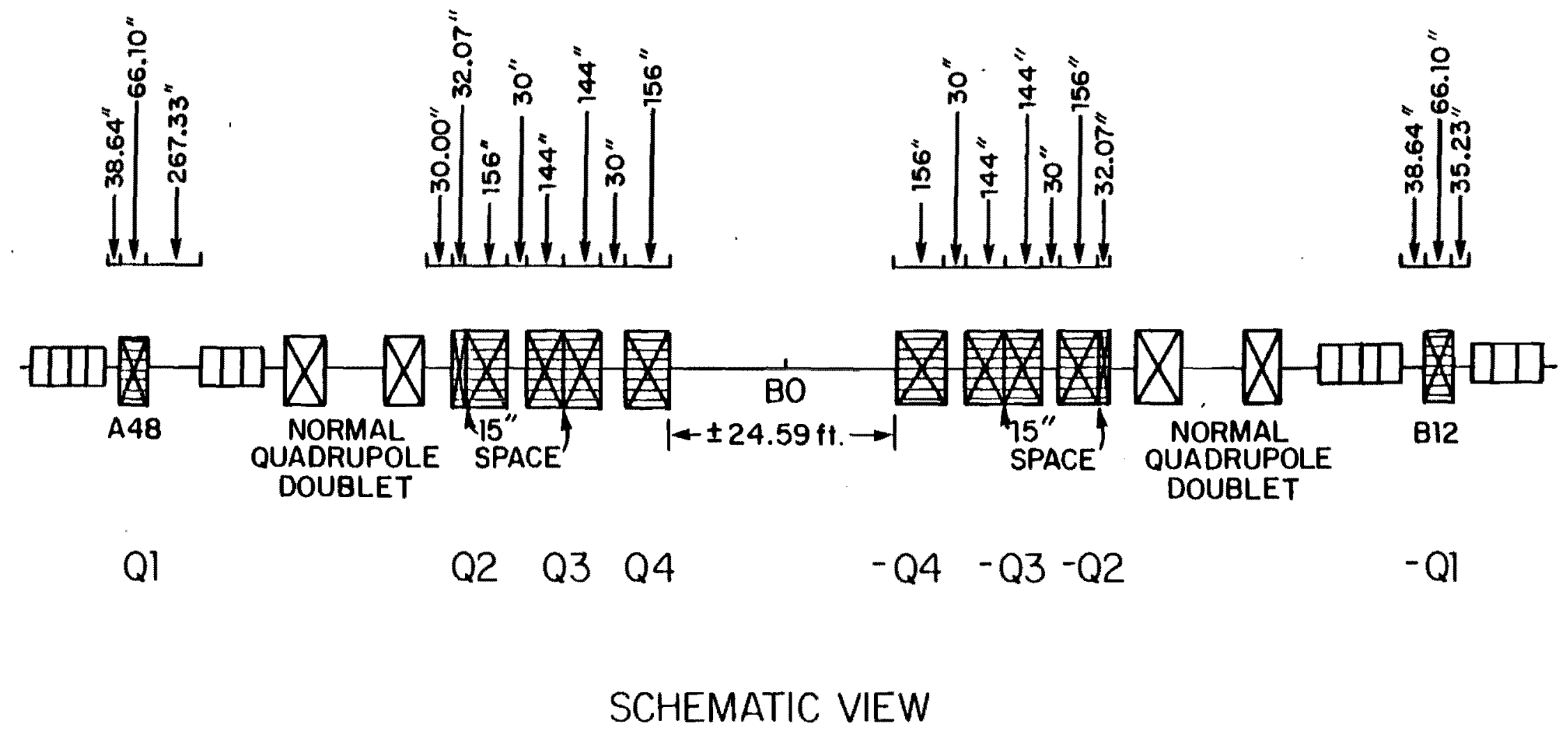

Fig. 7-1 
$\begin{array}{lllllllllllll}0 & 10 & 20 & 30 & 40 & 50 & 60 & 70 & 80 & 90 & 100 & 110 & 120\end{array}$

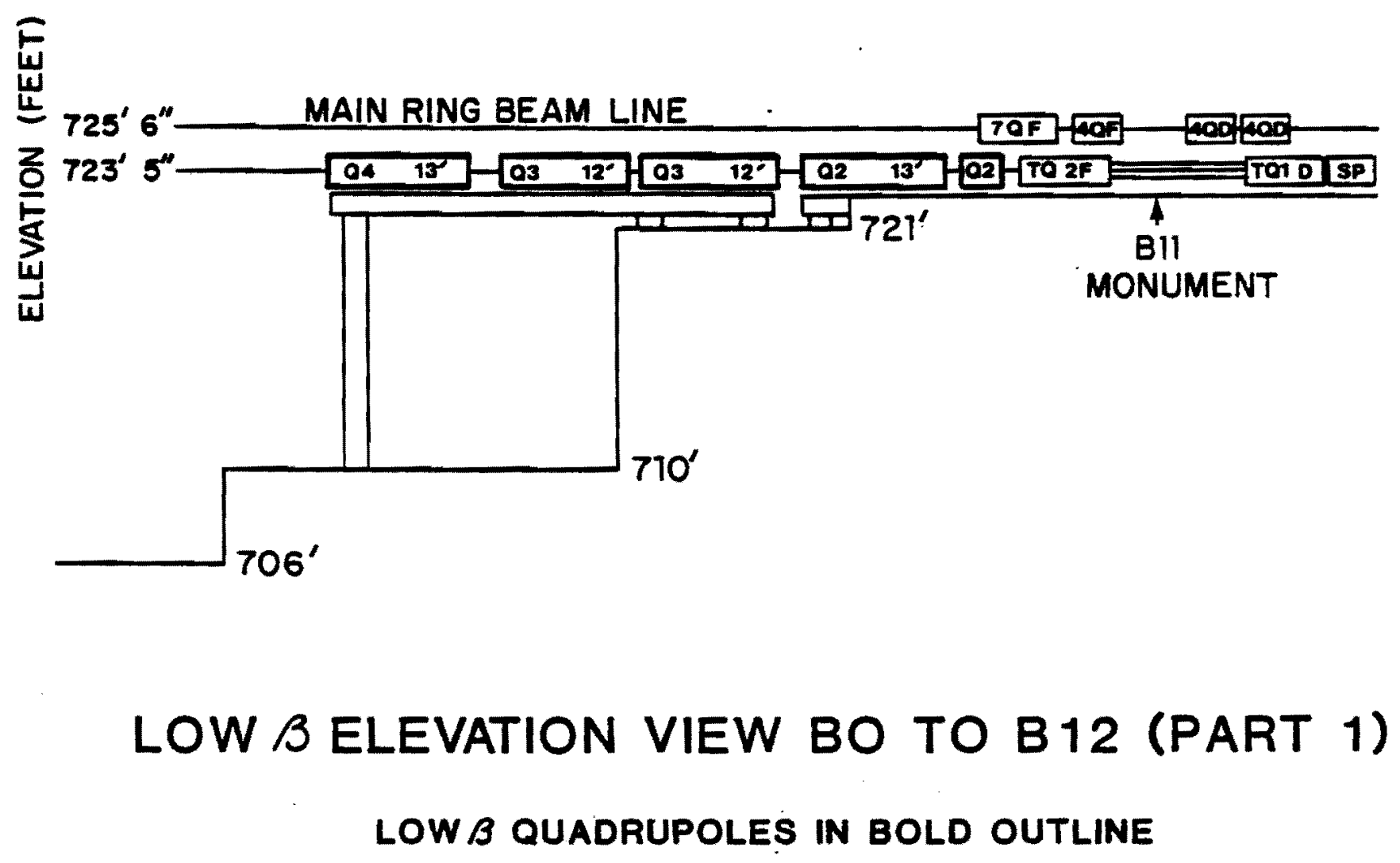

Fig. 7-2 
$120,130,140,150,160,170,180,190,200,210,220$ (FEET)

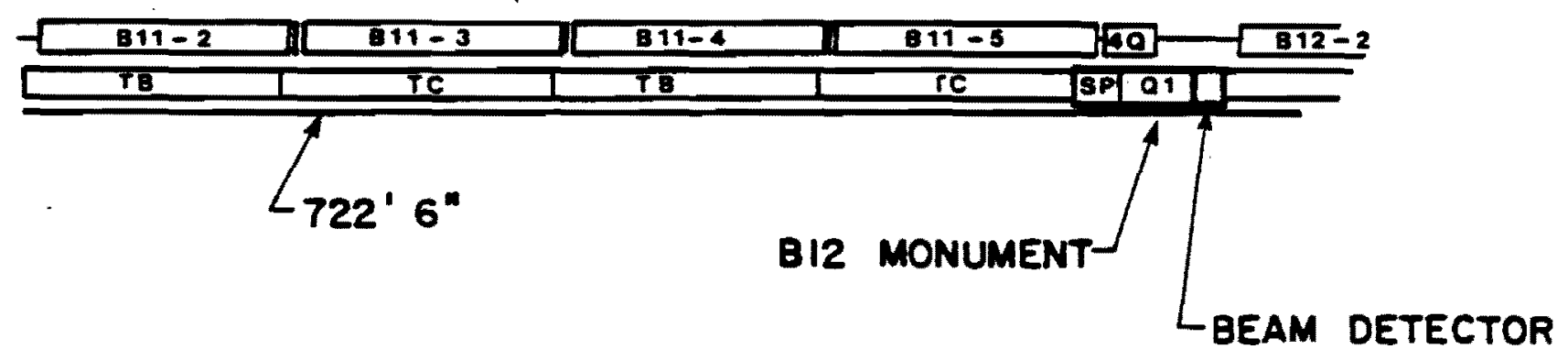

\section{LOW $\beta$ ELEVATION VIEW BO TO B 12 (PART 2)}

LOW $\beta$ QUADRUPOLES IN BOLD OUTLINE

Fig. $7-2$ 


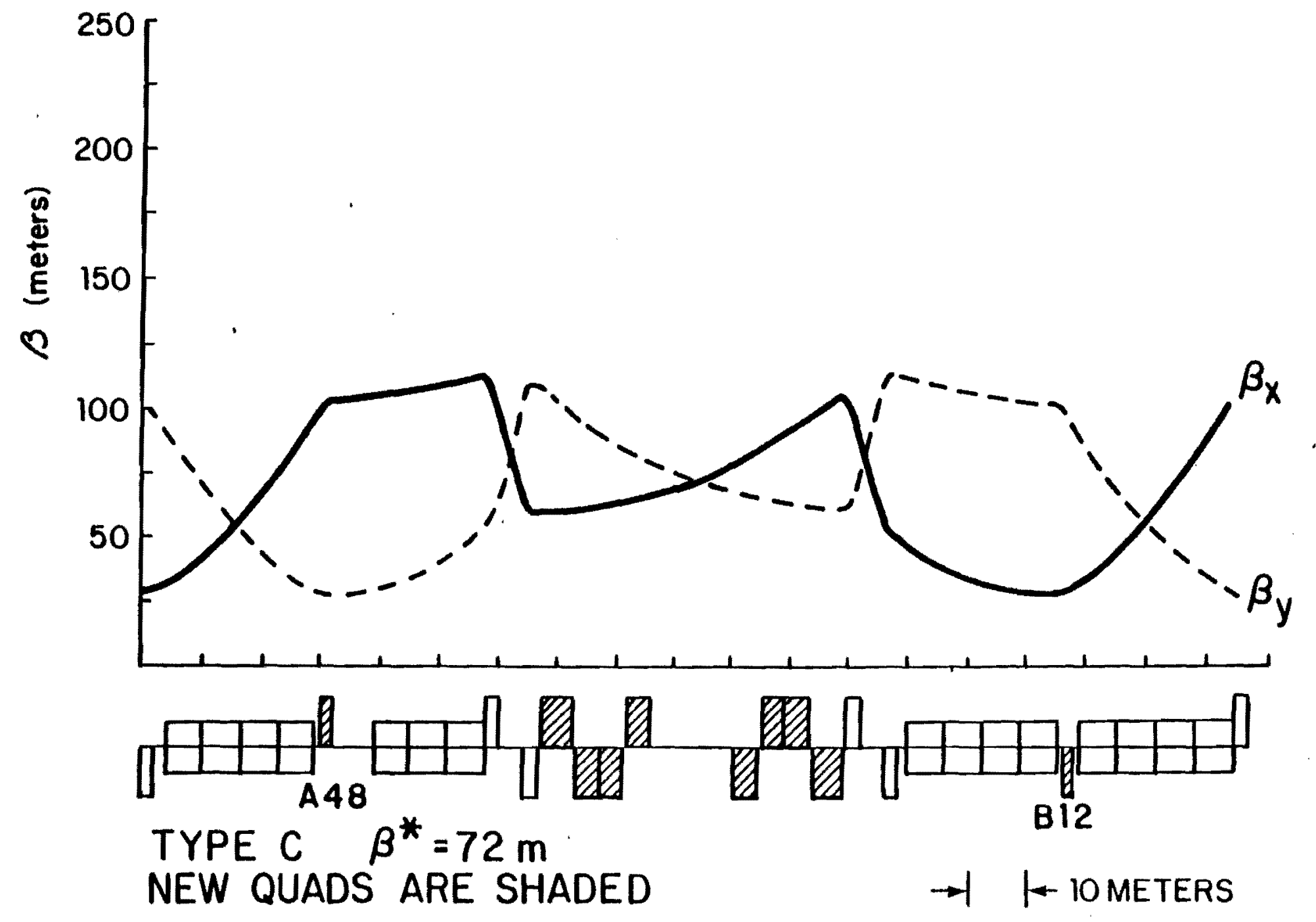

Fig. 7-3 


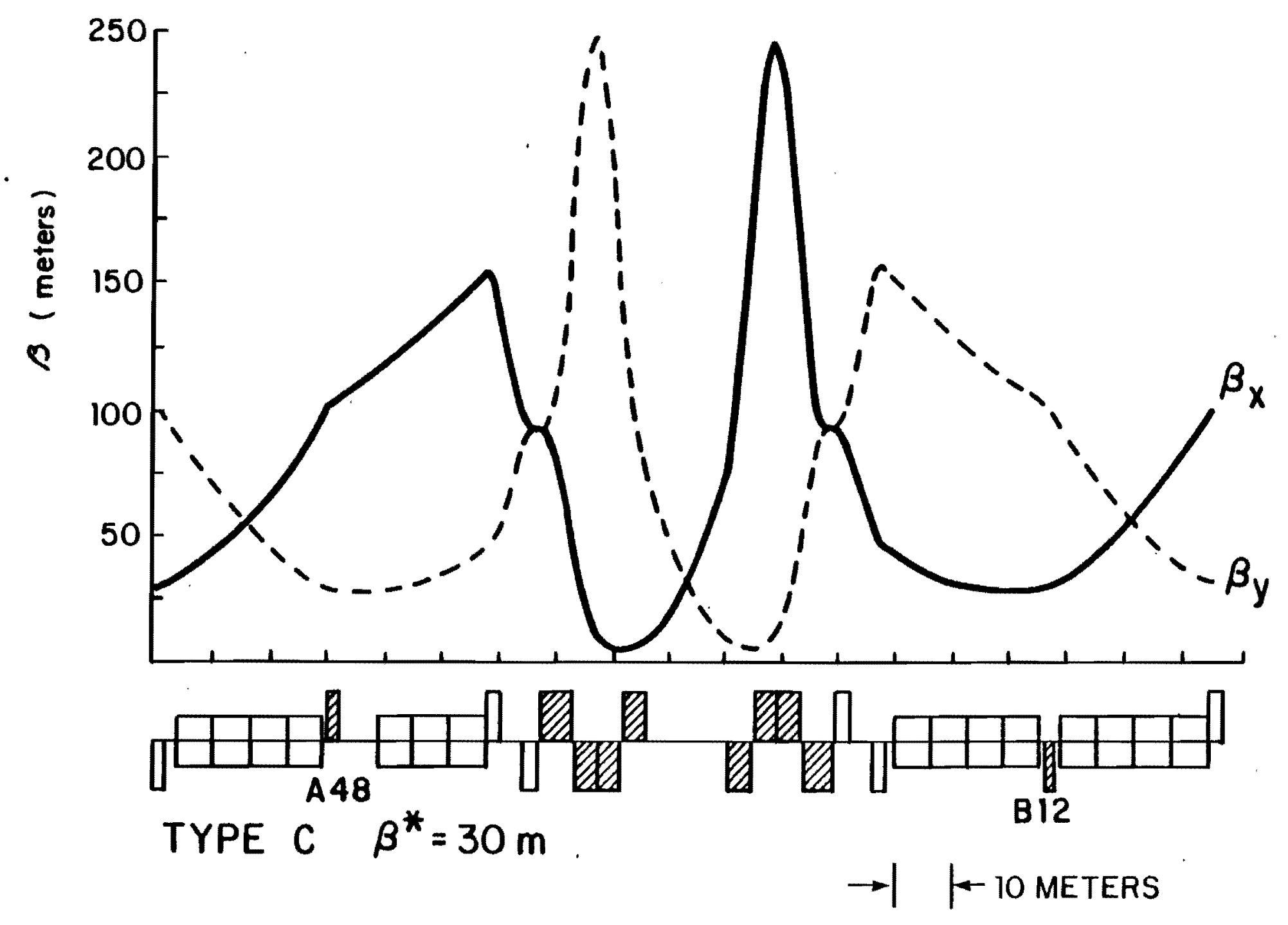

Fig. 7-4 


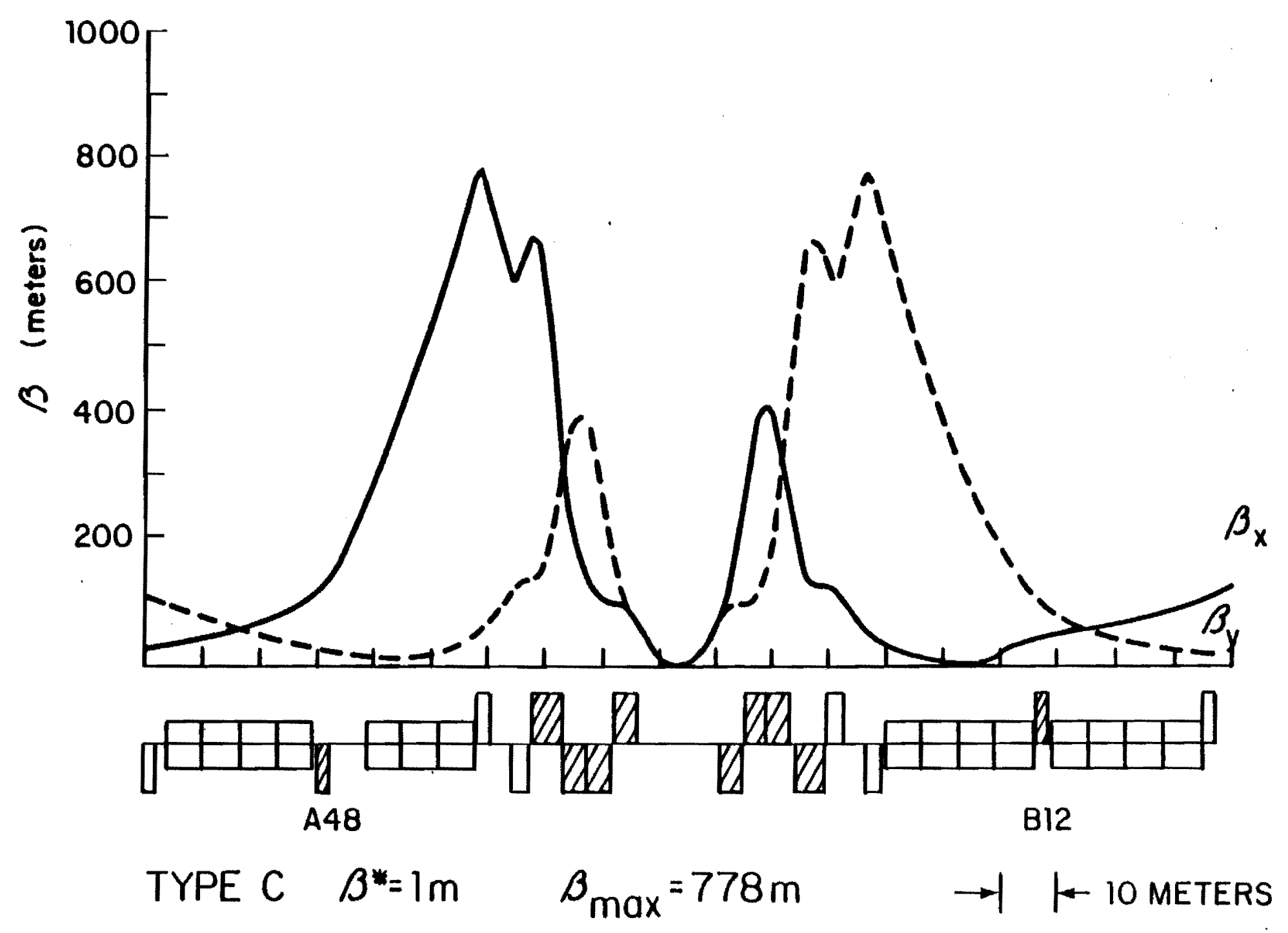


Beam Momentum Spread and Emittance Variation with Time Due to Intrabeam Scattering

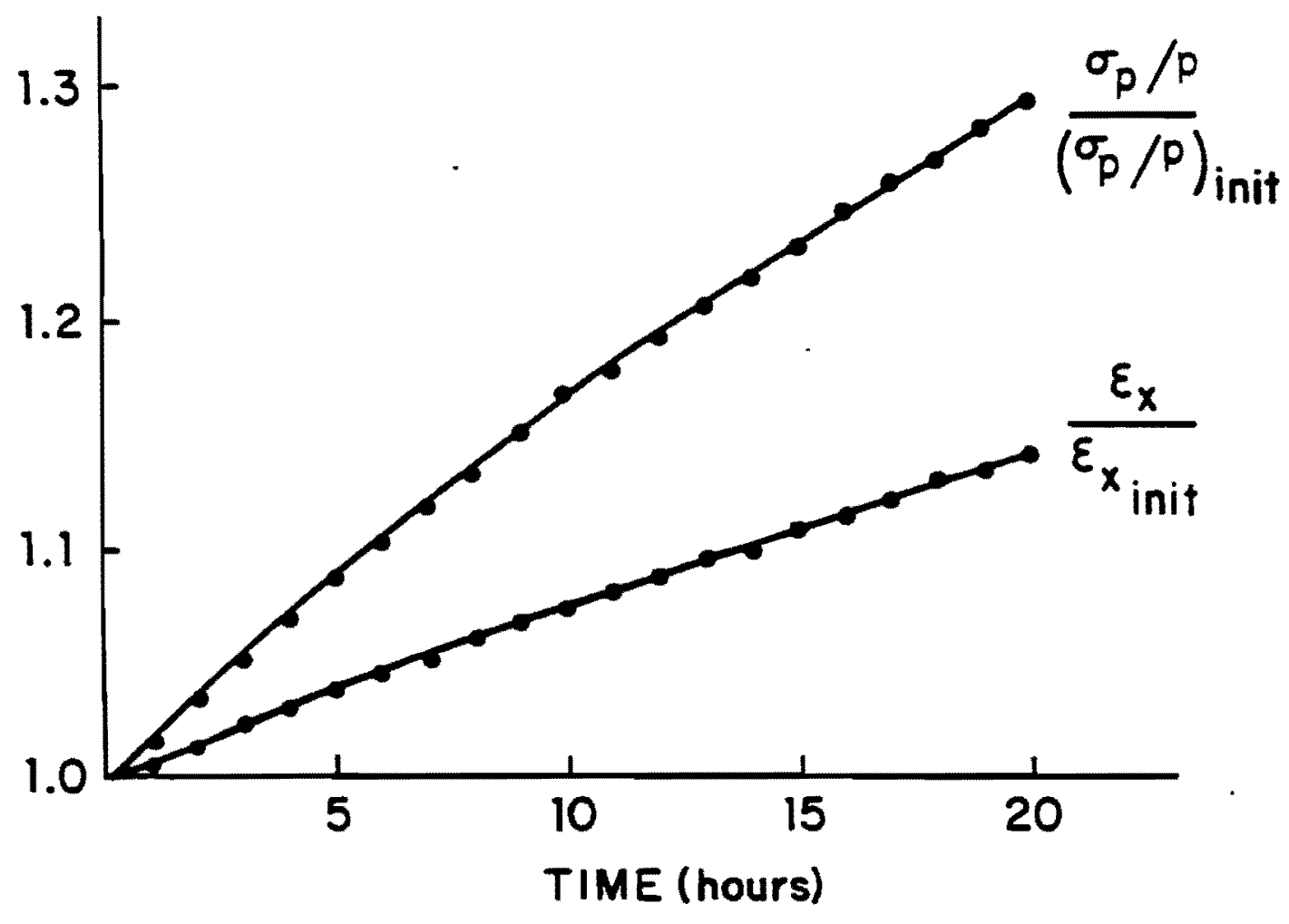

Fig. 8-1 

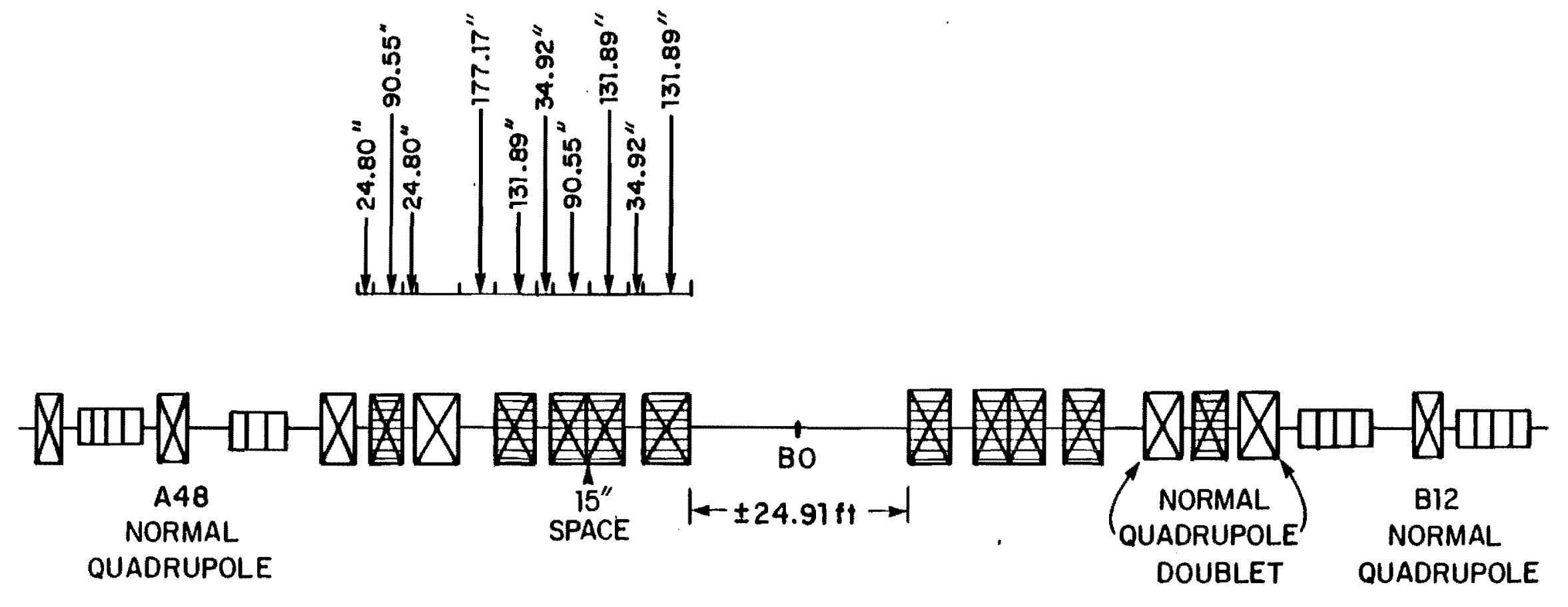

$\begin{array}{lllllll}\mathrm{Q} & \mathrm{Q} 2 & \mathrm{Q} 3 & \mathrm{Q} 4 & -\mathrm{Q} 4 & -\mathrm{Q3}-\mathrm{Q} 2 & -\mathrm{Q} 1\end{array}$

SCHEMATIC VIEW 


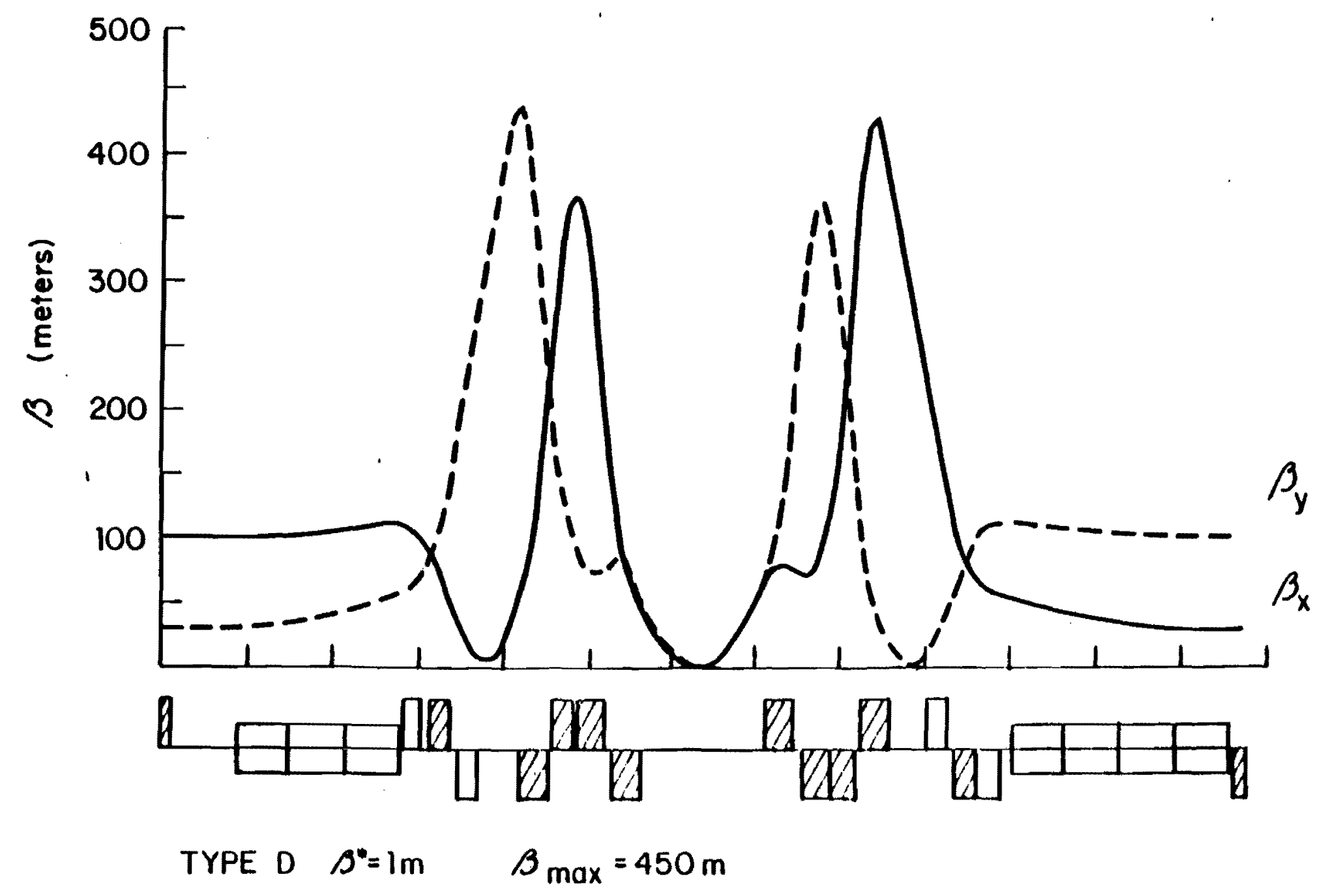

Fig. 10-2 